\title{
Development and Implementation of a Cost-effective Cockpit Interface Architecture for Flight Simulation
}

by

\section{Brent Andrew Cameron}

\author{
A thesis submitted to \\ the Faculty of Graduate and Postdoctoral Affairs
}

in partial fulfilment of

the requirements for the degree of

Master of Applied Science

in

Aerospace Engineering

Ottawa-Carleton Institute for Mechanical and Aerospace Engineering

Department of Mechanical and Aerospace Engineering

Carleton University

Ottawa, Ontario, Canada

June 2016

Copyright (C)

2016 - Brent Andrew Cameron 


\section{Abstract}

High-fidelity flight simulation has the potential to reduce the cost and increase the safety of ab-initio, or introductory, flight training. However, many existing flight simulation training devices either lack fidelity or are completely unaffordable for smaller flight schools and flying clubs. This thesis details the development and implementation of several cost-effective technologies suitable for use in low-cost flight training devices. Using these technologies, an initially-obsolete and non-functional Cessna 172 flight training device by Vector Training Systems, Inc. (circa 2002) was reverseengineered, redeveloped, modernized, and its functionality extended to create the Carleton University Redeveloped Vector Simulator (CURVS).

Throughout the design process, the use of robust, yet cost-effective commercialoff-the-shelf hardware and software was emphasized. The developed technologies included systems to interface to and control the existing cockpit systems such as analogue input (flight controls and knobs), digital input (switches and buttons), electric control loading servomotors, and simulated mechanical flight instruments. The X-Plane flight simulation application was used to replace the original legacy software, and was run on a network of custom-built, high-performance gaming computers. Additional technologies developed included an X-Plane-compatible simulation of the Garmin GNS 350W global navigation system, and an innovative, asymmetric wraparound projection system which increased situational awareness by affording an over-the-shoulder view to the pilot. The pre-existing circuit breaker and digital 
output relay systems were also successfully reverse-engineered, and work to interface them to X-Plane is ongoing.

Using modern commercial off-the-shelf hardware and software, this research showed that the potential exists for a new generation of high-fidelity yet cost-effective flight training devices to be created which are suitable for use by small flight training units. 
To my Lord and saviour Jesus Christ, for creating such an amazing world for us to enjoy, explore, and even fly in. 


\section{Acknowledgments}

The author is indebted to a great many people and organizations who contributed their time, documents, advice, encouragement, assistance, and funding towards the success of the CURVS project.

Jon Roloff, former lead electronics technician at Vector Training Systems, Inc. provided electronic copies of many pages of original simulator documentation which were invaluable to the reverse-engineering process.

Jared Wilhelmi, former instructor of Simulator Maintenance Technology at Lake Region State College in North Dakota provided many pages of documentation for the Piper Warrior flight training devices by Vector Training Systems, Inc. These documents were instrumental in the successful reverse-engineering of the controlloading system.

The author also gratefully acknowledges the financial support by the Ontario Centres of Excellence, Connect Canada, and Waterloo Wellington Flight Centre which made this project possible.

Despite a heavy course load and other part-time employment, laboratory assistant Karim Youssef provided countless hours of his time to the CURVS project, much of it on a volunteer basis, and was responsible for the successful design and implementation of the simulated GPS/CDI subsystem. He was always willing to lend a hand with whatever needed doing, and frequently acted on his own initiative to improve the simulator. 
Neil McFadyen, Supervisor of Computer Operations in the Department of Mechanical and Aerospace Engineering at Carleton University wrote the $\mathrm{C}++$ code for the digital input interface program, helped to reverse-engineer the relay subsystem on the original simulator, and was responsible for the excellent suggestion that LabJack DAQs be used for all I/O operations in addition to control loading. Thanks are also due for his prompt and helpful network support.

Bruce K. Johnston, Software and Network Specialist in the Department of Mechanical and Aerospace Engineering at Carleton University, prepared a number of surplus PCs for use in the author's research, and was especially helpful in resolving network issues.

Nagui Mikhail, Computer Systems and Physical Facilities Manager in the Department of Electronics at Carleton University kindly loaned the author a digital oscilloscope and anti-static wrist strap which were essential for reverse engineering the servo-driven simulated flight instruments.

Sean Follows, Lab Support Technician in the Department of Electronics at Carleton Univsity provided advice on the likelihood of successfully reverse-engineering the original radio stack communications protocol.

Stephen Maclaurin, PC System Analyst in the Department of Electronics at Carleton Unversity provided advice on interpreting the brushless servomotor COMMAND signal requirements.

Steve Truttmann, Laboratory Technologist in the Department of Mechanical and Aerospace Engineering at Carleton University also provided advice regarding the brushless servomotor COMMAND signals and how to generate them.

Steve Vamosi provided much helpful advice regarding the legacy technology originally used in the simulator, and on best practices regarding instrumentation and electronics in general.

Andras Fabian, developer of the HD Mesh Scenery v.3 add-on scenery package 
for X-Plane graciously allowed his work to be used free of charge for both research and commercial purposes in the context of this project, and freely provided advice on suitable computer system requirements.

Harry Zanin, proprietor of Flight Plus Software, Ltd. provided much helpful advice in regards to cost-effective projectors and innovative construction techniques for lowcost cylindrical projection screens using glass-reinforced plastic whiteboard material.

Thanks are also due to the staff of the Advanced Cognitive Engineering (ACE) Lab at Carleton University who were always ready to provide information, advice, and some of the most incredible lab tours available, especially Murray Gamble, (Technical Director of Modelling and Simulation), Anne Barr (Simulation Developer), and Andrew Staples (Simulation Developer).

Hooman Rajaee worked to redevelop several of the cueing systems on this project, especially the projection system, and developed the visually-stunning, high-fidelity X-Plane scenery for airport CYKF which in large part makes the simulator what it is today. Hooman also provided assistance in reverse-engineering the circuit breaker system and measuring the force output of the original control loading system.

Stefano Sandri was instrumental in the mechanical design and fabrication of the frame for the projection screen, and continued his volunteer contributions by designing a 3D-printed housing for the simulated magnetic compass, sheet metal box to house the new electronics, and also assisted in measuring the force output of the original control loading system.

Bradley Jung assisted in evaluating commercially-available simulated radio stacks to replace the unsupported proprietary system originally used in the simulator, and also provided much assistance in measuring the force output of the original control loading system. Bradley also provided extensive troubleshooting and setup work to get the selected replacement radio stack working. 
Nick Bourgeois provided much helpful advice regarding selection of PC components and stabilizing the redeveloped control loading system.

Major Mathew Parsons, Royal Canadian Air Force test pilot, provided much helpful advice, support, references, and even an equation to represent the skid and slip ball in a turn coordinator. It was always fun to talk aviation with you.

The Rockcliffe Flying Club provided access to a Cessna 172 aircraft, enabling the author to obtain a set of cockpit measurements and document the horizontal field of view available in the actual aircraft.

Tanner Poulin, the author's flight instructor at the Rockcliffe Flying Club, was also helpful in explaining the mechanical operation of the Cessna brake system so that it could be implemented accurately in the redeveloped simulator.

Chris Hobbs, pilot, aviation author, and ground school instructor at the Rockcliffe flying club provided access to his personal aircraft for test flights and also gave programming advice. He is also one of the few people I have ever met who is familiar with the legacy technology known as the transputer.

Thanks are due also to my supervisor, Dr. Rob Langlois, for providing guidance, yet also the freedom to guide my own research, and to Bob Connors, General Manager of Waterloo Wellington Flight Centre, for providing such an interesting real-world engineering problem to solve.

Austin Meyer, lead developer of the X-Plane flight simulation program, provided light-speed product support, usually replying to questions by email within mere minutes.

An especially big thank you is due to Jason Watkins and Christopher Teubert for their development and continued support of the open-source NASA X-Plane Connect Toolbox which was used extensively in this project.

Thanks also to my good friends, Jason and Kathleen Braden, for their constant support and encouragement. The numerous Sunday afternoon lunches in your home 
were a very welcome break from studies and research.

Last but certainly not least the author wishes to thank his parents, Allan and Judy Cameron for their unflagging support, food, and countless rides home from late night work sessions in the lab. Many thanks are also due to my sisters Amy and Shana, for listening to my ideas, helping calibrate the servo gauges, and reminding me that there is indeed life after simulation. 


\section{Table of Contents}

Abstract $\quad$ ii

Acknowledgments $\quad$ V

Table of Contents $\quad x$

List of Tables $\quad$ XV

List of Figures $\quad$ xvii

List of Acronyms $\quad$ xx

1 Introduction 1

1.1 Simulator Classification . . . . . . . . . . . . . . . 1

1.2 Project Background . . . . . . . . . . . . . . . . . . . 2

1.3 Project Rationale . . . . . . . . . . . . . . . . . . . . . . 3

1.4 Project Requirements . . . . . . . . . . . . . . . . . 4

1.5 Project Context . . . . . . . . . . . . . . 6

1.6 Thesis Overview . . . . . . . . . . . . . . . . . . . . . . . . . 8

2 Initial Evaluation of the VectorSim 9

2.1 Original Hardware Configuration . . . . . . . . . . . . . . . 10

2.2 First Power-up . . . . . . . . . . . . . . . . . 23 
2.3 Assessment and Decisions . . . . . . . . . . . . . . . . . . . 24

3 Selection of Flight Simulation Software 25

3.1 Flight Simulation Theory . . . . . . . . . . . . . 25

3.2 Flight Simulation Software Evaluation _ . . . . . . . . . . . . 27

3.2.1 Microsoft Flight Simulator $X$. . . . . . . . . . . . . 28

3.2.2 Lockheed Martin Prepar3D Version 2 . . . . . . . . . . . . . . 28

3.2.3 Laminar Research X-Plane 10 Global . . . . . . . . . . . . . 29

3.2.4 Open-Source FlightGear . . . . . . . . . . . . . . . . . . . . . 29

3.2 .5 Presagis FlightSIM . . . . . . . . . . . . . . . . . . . . . . . 29

3.2.6 RT Dynamics FixedWingLib . . . . . . . . . . . . . . . . . . 30

3.3 Pros and Cons of Evaluated Software . . . . . . . . . . . . 30

3.4 Final Software Selection . . . . . . . . . . . . . . . 31

3.5 Software Interface to X-Plane . . . . . . . . . . . . . . . . . 33

4 Design of the Cockpit Interface System 35

4.1 Interface Hardware Requirements . . . . . . . . . . . . . . . . 36

4.2 Selection of Interface Hardware . . . . . . . . . . . . . . . . 36

4.3 Initial Design of System Architecture . . . . . . . . . . . . . . . . . . 41

4.4 Revised Design of System Architecture . . . . . . . . . . . . . . . . . 43

5 Reverse-engineering and Redevelopment of Major Subsystems 46

5.1 Available Documentation . . . . . . . . . . . . . . . . 47

5.2 Analogue Input . . . . . . . . . . . . . . . . . . . . . . . . . . . 48

5.2.1 Early Experiments on the Analogue Input Subsystem . . . . . 49

5.2.2 Interfacing to the Existing Analogue Input Subsystem . . . . . 52

5.2 .3 Analogue Input Interface Program _ . . . . . . . . . . . 55

5.2.4 Analogue Input Calibration Program . . . . . . . . . . . . . . 58 
5.3 Image Generation Computers . . . . . . . . . . . . . . . . . . 62

5.3.1 Recommended System Requirements . . . . . . . . . . 66

$5.3 .2 \quad$ Part Selection . . . . . . . . . . . . . . . . . . 67

5.3.3 Simultaneous Power-on . . . . . . . . . . . . . . . . . . . . 69

5.3.4 Performance Metrics . . . . . . . . . . . . . . . . . . 70

5.4 Control Loading . . . . . . . . . . . . . . . . . . . . . . . 73

5.4.1 Reverse-engineering the Control Loading System . . . . . . . . 80

5.4.2 Testing the Existing Control Loading Motors . . . . . . . . . . 81

5.4.3 Quantifying Control Loading Motor Output Torques ... . . 85

5.4.4 Initial Software Interface to the Control Loading System _ . . 87

5.4 .5 Stability Issues $\ldots \ldots \ldots$

5.4.6 Hypothesized Causes of Stability Issues. . . . . . . . . . . 89

5.4.7 Proposed Solution to Stability Issues _ . . . . . . . . . . . 91

5.5 Servo-based Instruments . . . . . . . . . . . . . . . . . . . 93

5.5.1 Reverse-Engineering the Existing Servo Instruments . . . . . . 94

5.5 .2 Initial Servo Instrument Interface . . . . . . . . . . . . 96

5.5.3 Final Servo Instrument Interface _ . . . . . . . . . . 100

$5.6 \quad$ Stepper-based Instruments . . . . . . . . . . . . . . . . . . 106

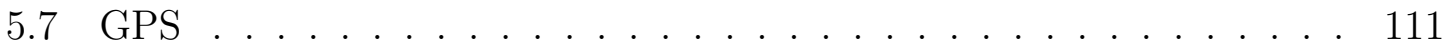

5.8 Digital Input $\ldots \ldots \ldots \ldots$

5.9 Radio Stack . . . . . . . . . . . . . . . . . . . . . . . . 120

5.10 Course Deviation Indicator . . . . . . . . . . . . . . . . . . 121

5.11 Circuit Breakers . . . . . . . . . . . . . . . . . . . 123

5.11.1 Reverse-engineering the Circuit Breaker Subsystem . . . . . 125

5.11.2 The Process of Tripping Circuit Breakers . . . . . . . . . . 128

5.11 .3 Circuit Breaker Datarefs . . . . . . . . . . . . . . . . 131 


\section{Supporting Systems}

6.1 Projection System . . . . . . . . . . . . . . . 133

6.1.1 Cylindrical Projection Screen . . . . . . . . . . . . . . 135

6.1 .2 Image-warping Software . . . . . . . . . . . . . . . 136

6.1.3 Custom Scenery for CYKF . . . . . . . . . . . . . . . 137

6.2 Audio ................................. 138

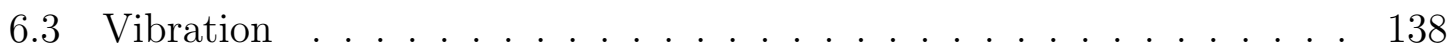

7 CURVS: The Big Picture 140

7.1 CURVS Startup . . . . . . . . . . . . . . . . 140

7.2 A Typical Simulation Session _. . . . . . . . . . . . . . . . 142

7.3 CURVS Shutdown . . . . . . . . . . . . . 146

8 Conclusion $\quad 147$

8.1 System-by-system Assessment . . . . . . . . . . . . . . . . . 147

8.1.1 Analogue Input System . . . . . . . . . . . . . . . . . . . . . . 147

8.1.2 Analogue Input Calibration . . . . . . . . . . . . . . . . . 148

8.1.3 Image Generation Computers . . . . . . . . . . . . . . . 148

8.1.4 Control Loading . . . . . . . . . . . . . . . . . . . . . . . . . . 148

8.1.5 Servo-based Flight Instruments . . . . . . . . . . . . . . . . . 149

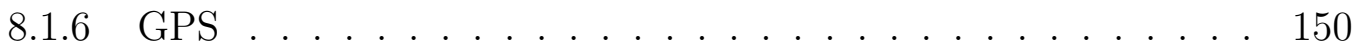

8.1 .7 Digital Input . . . . . . . . . . . . . . . . 150

8.1.8 Radio Stack . . . . . . . . . . . . . . . . . . 151

8.1 .9 Supporting Systems . . . . . . . . . . . . . . . . . 151

8.2 Cost Analysis . . . . . . . . . . . . . . . . . . . 153

8.3 Contributions .......................... 154

8.4 Future Work . . . . . . . . . . . . . . . . . . . . 157

8.5 Epilogue . . . . . . . . . . . . . . . . . . 159 
Appendix A History and Present Status of Diamond-Vector FTDs 173

A.1 Further Reading . . . . . . . . . . . . . . . . 175

Appendix B "Teensy" Microcontroller Joystick Interface Code 176

Appendix C X-Plane UDP Datagram Output Format 178

C.1 C\# and UDP . . . . . . . . . . . . . . . . . . . 179

C.2 Further Reading . . . . . . . . . . . . . . . . 180

Appendix D X-Plane UDP Dataset Variables 181 


\section{List of Tables}

2.1 Original VectorSim simulated instruments. . . . . . . . . . . 15

2.3 VectorSim OEM avionics. . . . . . . . . . . . . . 16

2.5 Simulated modules contained in the VectorSim radio stack. . . . . . . 17

3.1 Flight simulation software evaluated. . . . . . . . . . . . . 28

3.2 Pros and cons of flight simulation software evaluated. . . . . . . . . . 30

3.3 Prepar3D vs. X-Plane . . . . . . . . . . . . . . . . . . . 32

4.1 Interface hardware requirements. . . . . . . . . . . . . 36

4.3 Potential interface devices. . . . . . . . . . . . . . . . . . . . . 37

5.1 Analogue input sensors . . . . . . . . . . . . . . . 53

5.3 Recommended X-Plane system requirements. . . . . . . . . . . . 66

5.4 CURVS PC component list. . . . . . . . . . . . . . . 69

5.5 CURVS PC benchmarks (3DMark Fire Strike v. 1.1 ) . . . . . . . 70

5.6 Approximate X-Plane RAM usage. . . . . . . . . . . . . . . . 71

5.8 X-Plane stick force datarefs. . . . . . . . . . . . . . . . . . . . 77

5.10 Original vs. new servo calibration points for the airspeed indicator. . 105

5.11 X-Plane switch datarefs . . . . . . . . . . . . . . . . . . . 117

5.13 CURVS switch positions. . . . . . . . . . . . . . 118

5.15 Ignition switch truth table. . . . . . . . . . . . . . . 119

5.17 VectorSim circuit breaker specifications. . . . . . . . . . . . . . . 124

5.18 VectorSim circuit breaker mappings . . . . . . . . . . . . 126 
5.19 VectorSim circuit breaker test pin voltages. . . . . . . . . . . . . . . . 127

5.20 LabJack T7 DIO logic levels. . . . . . . . . . . . . . . . . . . 128

5.21 VectorSim circuit breaker truth table. . . . . . . . . . . . . . . 130

7.1 CURVS startup checklist. . . . . . . . . . . . . . . . 141

7.3 CURVS shutdown checklist. . . . . . . . . . . . . . 146

C.1 X-Plane dummy value in byte form . . . . . . . . . . . . . 178

C.2 X-Plane datagram structure . . . . . . . . . . . . . . . . 179

C.3 X-Plane datagram prologue . . . . . . . . . . . . . . . . . . . 179 


\section{List of Figures}

1.1 FTD vs. FSS: Cost vs. performance. . . . . . . . . . . . . . 7

2.1 Original configuration of VectorSim. . . . . . . . . . . . . 10

2.2 VectorSim at the ADL. . . . . . . . . . . . . . . . 11

2.3 Original VectorSim system diagram. . . . . . . . . . . . . 12

2.4 Original VectorSim visuals. . . . . . . . . . . . . . 13

2.5 VectorSim cockpit. . . . . . . . . . . . . . . . . 14

2.6 VectorSim potentiometers. . . . . . . . . . . . . 18

2.7 VectorSim brake box. . . . . . . . . . . . . . . . . 19

2.8 VectorSim control loading motors and drives. . . . . . . . . . . . 20

2.9 Control loading terminal strip. . . . . . . . . . . . . . 20

2.10 Cessna 172 control surfaces. . . . . . . . . . . . . . . . . . . . 21

2.11 Snap-on relays and DO-IF board . . . . . . . . . . . . . . 22

3.1 Flight simulation theory. . . . . . . . . . . . . . . 25

4.1 An interface device as a bridge. . . . . . . . . . . . . . . 35

4.2 The BeagleBone Black as an interface device. . . . . . . . . . . 39

4.3 LabJack T7 with LJTick-DAC modules. . . . . . . . . . . . . . . . . 40

4.4 CURVS initial system architecture. . . . . . . . . . . . . 42

4.5 CURVS revised system architecture. . . . . . . . . . . 45

5.1 Reverse-engineering the VectorSim. . . . . . . . . . . . . 47

5.2 Potentiometer circuit. . . . . . . . . . . . . . . . . 49 
5.3 Teensy microcontroller interface schematic. . . . . . . . . . . . . 50

5.4 Teensy microcontroller. . . . . . . . . . . . . . . . . . . 51

5.5 VectorSim connected to FlightGear. . . . . . . . . . . . . . . 52

5.6 Custom analogue input patch cable. . . . . . . . . . . . . 54

5.7 Construction of analogue input patch cable. . . . . . . . . . . . 54

5.8 Analogue input program flowchart . . . . . . . . . . . . 56

5.9 Linear interpolation . . . . . . . . . . . . . . . 57

5.10 Potentiometer drift. . . . . . . . . . . . . . . . . . . . . 59

5.11 CURVS analogue input calibration flowchart. . . . . . . . . . . 61

5.12 Computer graphics view frustum. . . . . . . . . . . . 63

5.13 Single-frustum vs. triple-frustum display systems. . . . . . . . . . . . 64

5.14 Test of MultiView X-Plane plugin. . . . . . . . . . . . . 66

5.15 X-Plane rendering settings. . . . . . . . . . . . . . 72

5.16 Generating stick force based on airspeed. . . . . . . . . . . . . . 77

5.17 X-Plane control force settings in Plane-Maker. . . . . . . . . . . . 78

5.18 Original VectorSim control loading system architecture. . . . . . . 81

5.19 Additional circuitry used to test the control loading motors. . . . . . 83

5.20 Control loading test apparatus. . . . . . . . . . . . . . . . . . . . 84

5.21 Control loading test apparatus. . . . . . . . . . . . . 86

5.22 Command voltage vs. output control force. . . . . . . . . . . 87

5.23 Proposed control loading algorithm with rate-triggered damping. . . . 93

5.24 Example VectorSim servo. . . . . . . . . . . . . . . . . . 94

5.25 Example servo signal traces. . . . . . . . . . . . . . . . . . 95

5.26 Servo instrument endstop. . . . . . . . . . . . . . . . 95

5.27 Airspeed indicator servo pulses. . . . . . . . . . . . . . 96

5.28 BBB servo control. . . . . . . . . . . . . . . . . . . . 97

5.29 X-Plane UDP dataset selection. . . . . . . . . . . . . . . . . . 99 
5.30 Low-cost USB range extender. . . . . . . . . . . . . . . . . . . . 101

5.31 CURVS servo instrument interface program flowchart. . . . . . . . . . 103

5.32 Detail of CURVS "update instrument" sub-process. . . . . . . . . . . 104

5.33 VectorSim servo-driven airspeed indicator. . . . . . . . . . . . . . 104

5.34 Non-linearity of airspeed indicator. . . . . . . . . . . . . . 105

5.35 Simple 2-pole PM stepper motor. . . . . . . . . . . . . . . . . . 107

5.36 Bipolar stepper motor schematic. . . . . . . . . . . . . . . 107

5.37 Slotted-disc and Optoschmitt sensor on the ADF. . . . . . . . . . . . 109

5.38 Stepper motor instrument interface flowchart. . . . . . . . . . . . . 110

5.39 Bendix-King KLN89B GPS. . . . . . . . . . . . . . . . . . 111

5.40 Emuteq GarSIM GNS530 compared with the Garmin GNS 530W . . . 113

5.41 VectorSim switches and indicators. . . . . . . . . . . . 115

5.42 CURVS digital input program flowchart. . . . . . . . . . . . . 115

5.43 Generic pullup resistor circuit. . . . . . . . . . . . . . . . . . . . 119

5.44 Original VectorSim radio modules. . . . . . . . . . . . . . 120

5.45 Bendix-King KI 209A CDI. . . . . . . . . . . . . . . . . . . 121

5.46 Bendix-King KI 209A CDI disassembled. . . . . . . . . . . . . . . . 123

5.47 KATIF-2 Circuit Breaker Circuitry. . . . . . . . . . . . . . . . . . 124

5.48 KATIF-2 circuit breaker LED bar. . . . . . . . . . . . . . . . . 127

6.2 Single-chip DLP colour wheel. . . . . . . . . . . . . . . . . . 134

6.3 CURVS projection screen. . . . . . . . . . . . . . . 135

6.4 Comparison of unwarped vs. warped images on a curved screen. . . . 136

6.5 Custom scenery for CYKF. . . . . . . . . . . . . . . . . 137

7.1 WWFC at CYKF. . . . . . . . . . . . . . . . . . 142

7.2 CURVS Cockpit. . . . . . . . . . . . . . . 145

8.1 The CURVS FTD. . . . . . . . . . . . . . . . . . 160

A.1 Original DA-20 Katana FTDs by Diamond Aircraft. . . . . . . . . . . 174 


\section{List of Acronyms}

\begin{tabular}{ll}
\hline Acronyms & Definition \\
\hline ADL & Applied Dynamics Lab \\
ADF & Automatic Direction Finder \\
AIN & Analogue Input \\
BBB & BeagleBone Black \\
CAT6 & Category 6 \\
CAD & Canadian Dollar \\
CB & Circuit Breaker \\
CDI & Course Deviation Indicator \\
COTS & Commercial-off-the-shelf \\
CMND & Command \\
CSV & Comma-Separated Values \\
CURVS & Carleton University Redeveloped Vector Simulator \\
CUSP & Carleton University Simulator Project \\
CYKF & Region of Waterloo International Airport (ICAO airport code) \\
DAC & Digital-to-Analogue Converter \\
DAQ & Data Acquisition [Device] \\
DC & Direct Current \\
DIDO & Digital Input / Digital Output \\
DIO & Digital Input/Output \\
DLL & Dynamic Link Library \\
DLP & Digital Light Processing \\
& \\
\hline
\end{tabular}


DME Distance Measuring Equipment

EFB Electronic Flight Bag

FFS Full-Flight Simulator

FPS Frames per Second

FTD Flight Training Device

GBP Great Britain Pound

GPIO General-Purpose Input/Output

GPS Global Positioning System

GS Glideslope

GUI Graphical User Interface

HD High Definition

HDD Hard Disk Drive

$\mathrm{Hz} \quad$ Hertz

I/O Input/Output

IC Integrated Circuit

ICAO International Civil Aviation Organization

IDE Integrated Development Environment

IFR Instrument Flight Rules

ILS Instrument Landing System

IOS Instructor Operator Station

IP Internet Protocol

JIT Just-in-time

KATIF Katana Interface

KIAS Knots Indicated Airspeed

kts knots (nautical miles per hour)

LAN Local Area Network

LCD Liquid Crystal Display

LED Light-emitting Diode

LOC Localizer

LPF Low Pass Filter 


\begin{tabular}{|c|c|}
\hline $\mathrm{ms}$ & Millisecond \\
\hline NASA & National Aeronautics and Space Administration \\
\hline $\mathrm{NAV} / \mathrm{COM}$ & Navigation/Communication \\
\hline OBS & Omni Bearing Selector \\
\hline Op-Amp & Operational Amplifier \\
\hline $\mathrm{PC}$ & Personal Computer \\
\hline $\mathrm{PM}$ & Permanent Magnet \\
\hline PPL & Private Pilot License \\
\hline RAM & Random-Access Memory \\
\hline RGB & Red, Green, Blue \\
\hline $\mathrm{RPM}$ & Revolutions per Minute \\
\hline SDK & Software Development Kit \\
\hline SSD & Solid-State Drive \\
\hline UDP & User Datagram Protocol \\
\hline USB & Universal Serial Bus \\
\hline USD & United States Dollar \\
\hline V & Volt \\
\hline VDC & Volts Direct Current \\
\hline VOR & Very high frequency Omnidirectional Range \\
\hline VRAM & Video RAM \\
\hline VTS & Vector Training Systems, Inc. \\
\hline WWFC & Waterloo Wellington Flight Centre \\
\hline XML & Extensible Markup Language \\
\hline $\mathrm{XPC}$ & X-Plane Connect \\
\hline
\end{tabular}




\section{Chapter 1}

\section{Introduction}

The purpose of this thesis is to document the reverse-engineering, design, and development work which was performed in order to upgrade, improve, and return an initially-obsolete flight simulation training device to active service. Throughout this work, cost-effectiveness, robustness, and the use of commercial-off-the-shelf (COTS) components was emphasized.

\subsection{Simulator Classification}

The classification and certification of flight simulators in Canada is the responsibility of Transport Canada [1]. The Canadian classification system is compatible with those of both the International Civil Aviation Organization (ICAO), and the Federal Aviation Authority (FAA) in the United States of America [2-4]. For the purposes of certification in Canada, the two general classifications are:

- Aeroplane Simulator, consisting of levels A-D

- Flight Training Device (FTD), consisting of levels 1-7

Aeroplane simulators are representative of a specific aircraft, include motion cuing and an out-of-cockpit visual display system, and are held to high standards of 
fidelity with respect to the actual aircraft. On the other hand, flight training devices may be generic, do not require motion cuing, and require only that the flight model be representative of the general class of aircraft being simulated (e.g., single-engine piston-powered aircraft). Full requirements for both aeroplane simulators and FTDs may be found in the Aeroplane and Rotorcraft Simulator Manual (TP9685E) published by Transport Canada. Outside of Canada, a flight training device is generally known by the more modern ICAO term flight simulation training device (FSTD) [2].

An aeroplane simulator or FTD which has been certified by Transport Canada may be used to log a certain amount of training time towards aircraft pilot licences and ratings. This time is counted as equivalent to time spent in an actual aircraft.

\subsection{Project Background}

This project was a joint venture between the Carleton University Applied Dynamics Laboratory and Waterloo Wellington Flight Centre (WWFC). WWFC is based at the Region of Waterloo International Airport in Breslau, Ontario, and is one of the largest flight training schools in Canada, with a fleet of 22 aircraft. Funding was provided by both WWFC and the Government of Ontario through the TalentEdge Internship Program (TIP) offered by the Ontario Centres of Excellence (OCE). Funding was also provided by the Canadian federal government via Connect Canada, co-funder of the TalentEdge internship program.

In addition to their fleet of aircraft, WWFC operates two FTDs, the most recent being an Alsim ALX, along with an older Precision Flight Controls (PFC) Modular Flight Deck (MFD) [5,6].

Before these devices, WWFC had used a Victory 2 Single-Engine Land Flight Training Device by Vector Training Systems, Inc. (VTS) [7]. This device was designed to simulate a Cessna 172 Skyhawk aircraft. However, in the years since its acquisition, 
the original manufacturer had gone out of business, the FTD had broken down, become non-functional, and its computer systems had become obsolete. However, given the fact that the rental rate for the Alsim ALX is nearly the same as that of a Cessna 152 aircraft, WWFC had the need for an additional, more affordable FTD to augment its student pilot training programs [8].

The Applied Dynamics Laboratory (ADL) at Carleton University has prior research experience in the area of cost-effective flight simulation, namely through the KatanaSim project, in which an unserviceable Diamond DA-20 Katana aircraft was converted into a cost-effective full-motion flight simulator $[9,10]$.

For the purposes of this project, WWFC provided the ADL with the aforementioned non-functional Vector FTD, with the intention that it be overhauled, modernized, and returned to service in as cost-effective a manner as possible. In keeping with the naming convention established by the KatanaSim project, the original, unmodified Vector FTD will be referred to as the VectorSim. A significant amount of background research was conducted regarding the past history of the VectorSim, past

and current users of similar flight training devices, and the origins of Vector Training Systems itself. This investigation resulted in the establishment of useful contacts, including a former employee of VTS, and one current user of a VTS Piper Warrior. These individuals kindly contributed hundreds of pages of technical documentation, which were invaluable to reverse-engineering the simulator, and ultimately, the success of the project. The details of this "detective work" are contained in Appendix A.

\subsection{Project Rationale}

At the time of its manufacture in the year 2002, the VectorSim was an advanced flight training device, featuring a full suite of instrumentation and avionics representative 
of a well-equipped Cessna $172 \mathrm{~S}$ aircraft. In addition, the simulator featured electric control loading motors on all three flight control axes, which was a rarity for its time and original price point, and not actually required for certification as an FTD. WWFC had obtained Transport Canada certification for the device as a Level 3 FTD.

As the computer technology originally included in VectorSim was found to be obsolete, and technical documentation was not initially available, it was decided that instead of restoring the FTD to its original state (circa 2002), the FTD would instead be redeveloped, expanding its capabilities by utilizing the latest advances in computing, software, graphics, and interface hardware. In order to ensure cost-effectiveness and maintainability, use of pre-existing commercial-off-the-shelf hardware and software was emphasized.

In a playful recognition of its original beginnings, the modernized FTD is now known as the Carleton University Redeveloped Vector Simulator (CURVS).

Notice that while the innovative, cost-effective technologies and simulator interface architecture developed in this project were applied to the redevelopment of a specific FTD, this project was part of a long-term research program at the Carleton University Applied Dynamics Laboratory to develop cost-accessible flight simulation technologies for the purpose of ab-initio flight training. As such, the technologies designed and developed in this project are in many cases transferable to other applications and simulators, and not necessarily restricted to use with a single FTD.

\subsection{Project Requirements}

WWFC was consulted early on in the project to determine which usage scenarios and other basic requirements were essential for the redeveloped FTD. The new FTD was to be used for ab-initio pilot training, as well as instrument training and recertification. WWFC further specified that the finished product be capable of being certified 
by Transport Canada as a Level 2 FTD. It also became apparent that high-fidelity visuals and control loading, although not required by the Level 2 classification, were extremely important to the customer. FTDs incorporating such extras are unofficially referred to in the flight training industry as Level $2+$ devices.

From these consultations, a set of specific technical requirements was then developed. These requirements are listed below:

- Modern flight simulation software;

- State-of-the-art graphics;

- Functional mechanical gauges;

- Functional radio stack;

- Instructor Operator Station (IOS);

- Control Loading on all three axes;

- Functional GPS unit;

- Sound effects;

- Ethernet or other noise-resistant connections between computers and simulator;

- Multi-channel, wraparound visuals (full HD or better); and

- Comprehensive documentation:

- Operations manual;

- Backup media with re-installation instructions; and

- Full source code to allow for future maintenance and/or modification.

Several desirable features were also defined:

- Seat rumble transducers; and

- Enlarged cockpit windows 


\subsection{Project Context}

There are a wide variety of flight simulators available today, with varying capabilities, classifications, and costs. Full-flight simulators provide a high level of performance in terms of fidelity and immersion; however, they typically cost many millions of dollars. For example, the contract recently awarded for a new CAE 3000 Series helicopter simulator for the Canadian Coast Guard was worth 17,000,000 CAD [11,12].

Flight training devices, on the other hand, cost less but typically provide lower levels of performance. However, even lower-fidelity FTDs still feature a price tag which may be difficult or impossible for small flight schools to afford. For example, the cost of modern Level 2 FTDs range from tens of thousands of dollars (e.g., Redbird FMX at 64,800 USD) to hundreds of thousands of dollars (e.g., ALSIM ALX) [5]. In order to keep costs down, existing modern FTDs often make compromises in terms of both the visual system or type-specificity. For instance, the Redbird FMX, in addition to being a non-type-specific (Level 2) FTD, uses a semi-circle of computer monitors for the display, which breaks up the pilot's field of view, as well as positioning the image unrealistically close to the pilot. These compromises reduce both student immersion and transfer of training to an actual aircraft.

In contrast, the CURVS project created a modern type-specific (Level 5), highfidelity FTD with control loading and an uninterrupted $220^{\circ}$ wraparound display at a cost affordable by even small flight schools. The place of this new cost-accessible FTD in the modern flight simulation arena is illustrated graphically in Figure 1.1. 


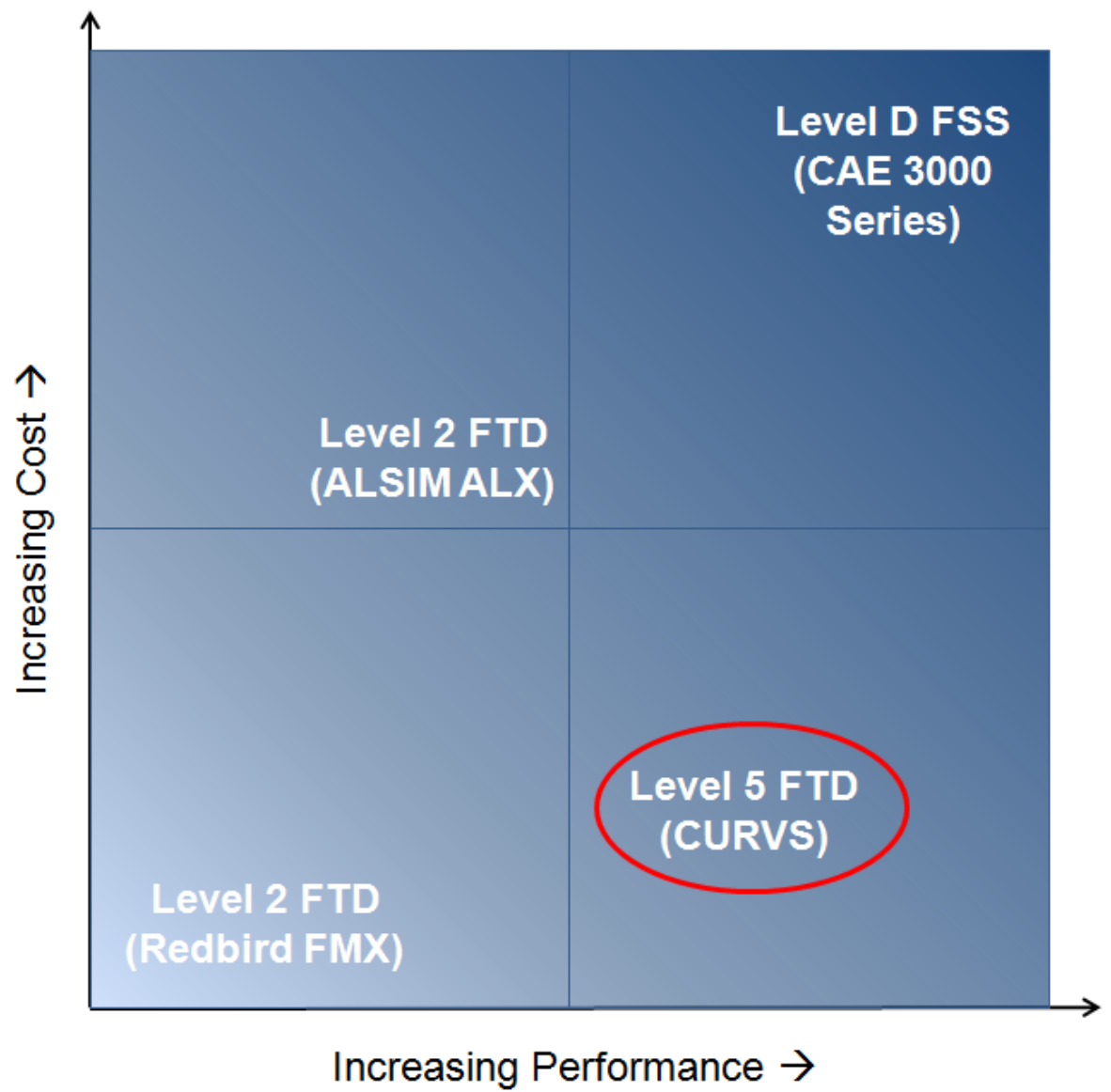

Figure 1.1: Qualitative comparison of cost vs. performance for several modern simulators. The CURVS FTD was designed to fill a niche for high-performance yet low cost FTDs. 


\subsection{Thesis Overview}

Chapter 2 presents an evaluation of the state of the VectorSim FTD as received at the beginning of the project. Chapter 3 details the selection process used to select a modern flight simulation computer program to replace the obsolete software originally shipped with the FTD. Chapter 4 presents the new system architecture which was designed in order to interface the existing cockpit controls and instrumentation to new flight simulation computers. Chapter 5 details the process by which the pre-existing simulator subsystems were reverse-engineered, interfaced to X-plane if possible, or replaced with modern, cost-effective solutions where necessary. In order to give a complete picture of the redevelopment work performed to modernize and extend the capabilities of the original simulator, Chapter 6 provides a brief overview of the work performed by the author's colleague Hooman Rajaee in regards to various cueing systems. Chapter 7 provides a high-level overview of how the individual, redeveloped simulator subsystems worked together as a cohesive whole in the context of a typical simulation session. Chapter 8 concludes the thesis with a system-by-system assessment of the redeveloped simulator, and a discussion of specific contributions made towards the advancement of cost-effective flight simulation. Additional information which may be relevant to researchers interested in developing software for cost-effective flight simulation is included in the appendices. Appendix A contains a brief history of the Diamond/VTS line of FTDs. Example joystick interface code for the Arduino-compatible Teensy microcontroller appears in Appendix B. Appendix C details the byte-level format of X-Plane's UDP output datagrams. Finally, Appendix D provides a complete listing of the ordering of the contents of X-Plane's predefined UDP datasets. 


\section{Chapter 2}

\section{Initial Evaluation of the VectorSim}

In order to determine which components of the VectorSim flight training device were still functional, and thus available for reuse, an initial evaluation was conducted starting May 2014. The VectorSim flight training device was manufactured in February 2002, with model number P7600 and serial number 00. As it turned out, it was the first and only example of its type (Cessna 172) ever completed by Vector Training Systems, Inc. The original configuration of the VectorSim is shown in Figure 2.1. 


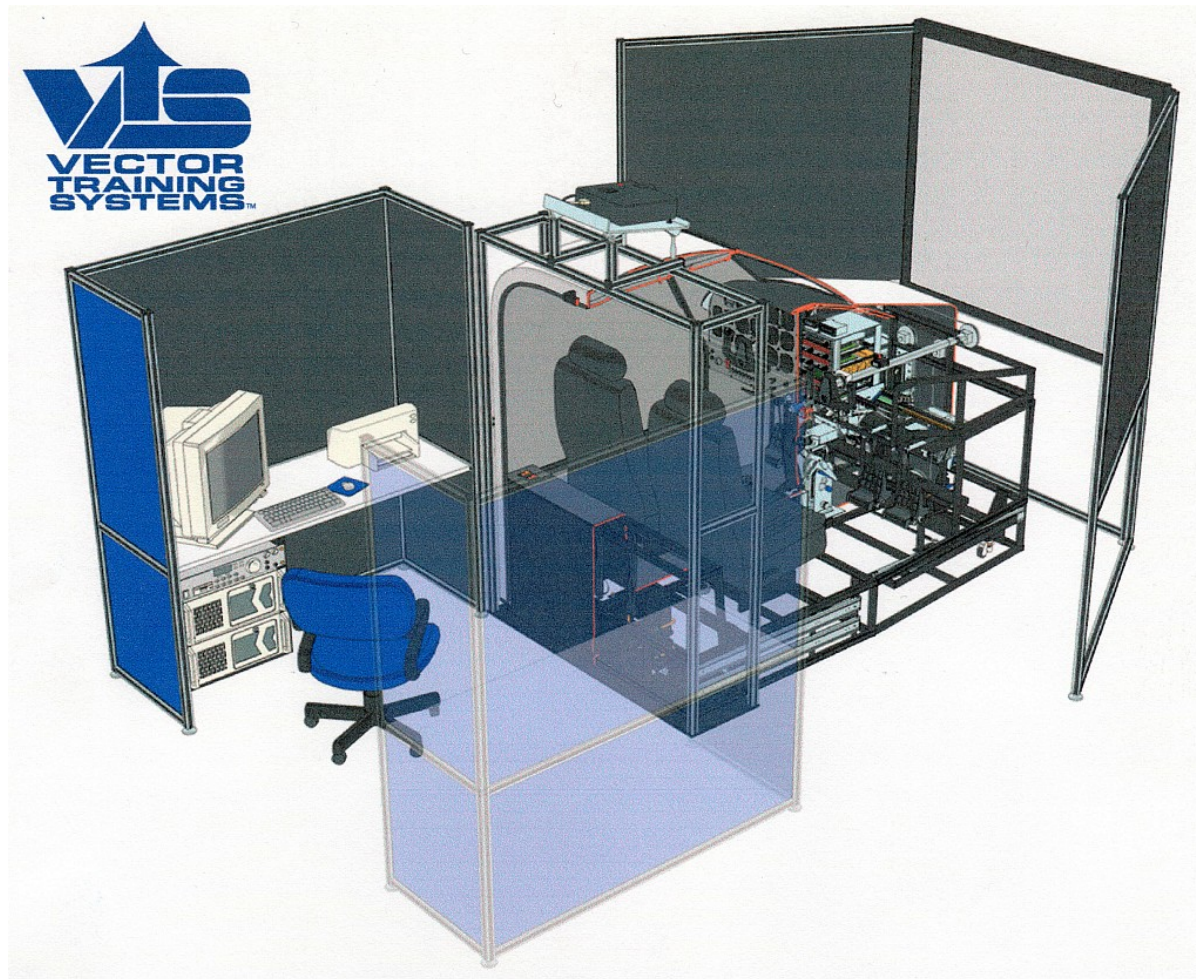

Figure 2.1: VectorSim flight training device as originally configured [13]. The white panel in front of the device is a projection screen for the projector mounted above the cockpit.

\subsection{Original Hardware Configuration}

The original hardware specifications were found in the Reference Data Report by Vector Training Systems [7]. This document was produced for certification purposes and accompanied the simulator. The VectorSim as it was originally received at the ADL is shown in Figure 2.2. 


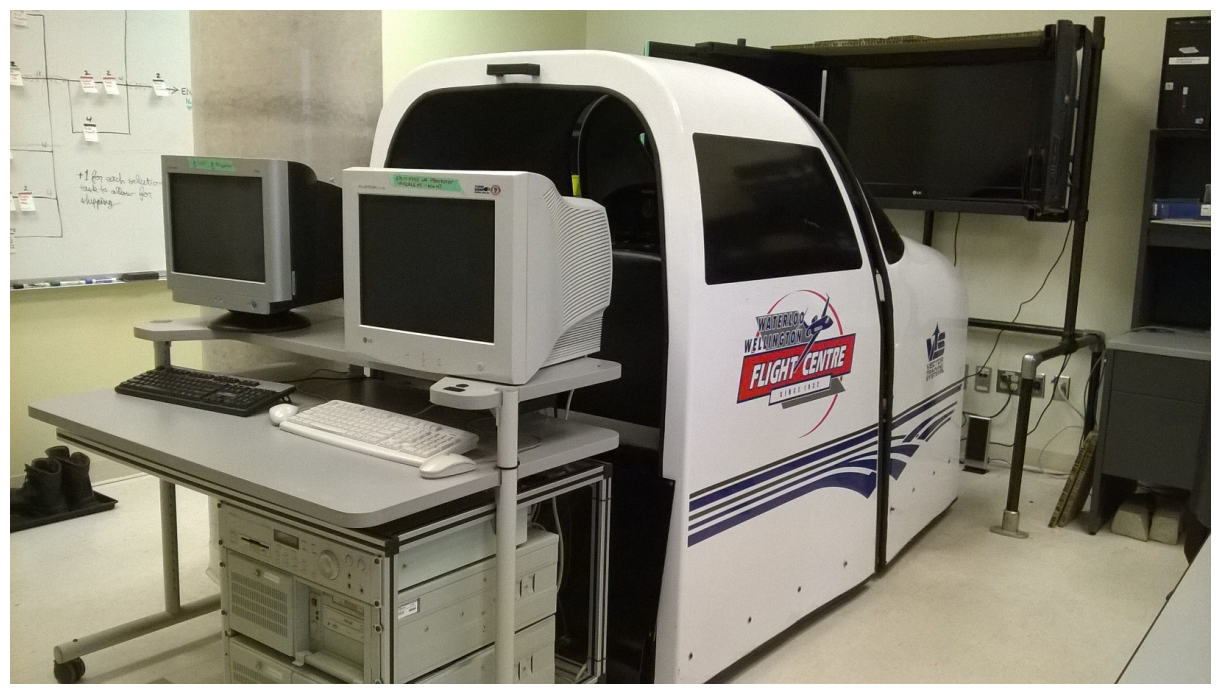

Figure 2.2: VectorSim flight training device as received at the ADL. Note that the flat-panel television array in front of the device was not part of the original simulator.

In contrast to most simulators of the present day, flight dynamics calculations were performed by a computer system installed within the simulator cockpit itself. This embedded computer consisted of a system of two SGS Thompson T/425-800 transputers (explained in more detail below) operating in parallel. The transputers featured a computation cycle time of less than 10 milliseconds, and were also responsible for administering all data acquisition and control operations required in order to interface to the cockpit sensors and avionics. Data acquisition and control were provided by additional cards for analogue output, analogue input, digital I/O, etc. These I/O cards were connected to a common backplane which was also shared by the two transputer cards. An original system diagram is shown in Figure 2.3.

The transputer, I/O, and other interface cards in the VectorSim were manufactured by Luftfahrtstudio Jungwirth, an Austrian company. Unfortunately, further research revealed that this company had declared bankruptcy in 2010 [15].

The transputer was an early form of parallel computing, first introduced by Inmos in 1985, which for a time found application in high-performance embedded 


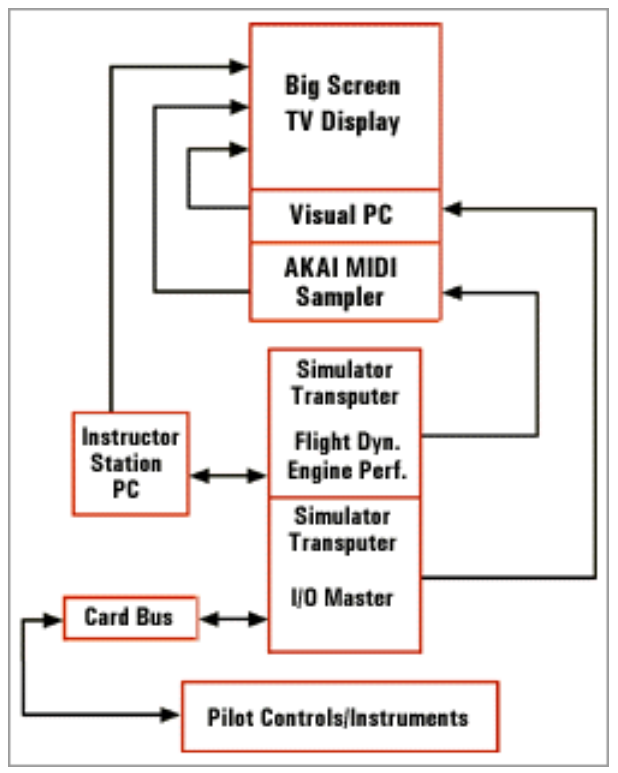

Figure 2.3: Original system diagram showing the use of two transputers. Image by VTS courtesy of Archive.org [14].

systems [16-18]. The name "transputer" is a portmanteau of transistor and microcomputer. Individual transputer chips were intended to be used as building blocks in the construction of complete parallel computers in much the same way that individual transistors were already being used to construct individual, standalone processors [19].

Two Intel Pentium III-based PCs resided in a separate rack outside of the simulator. One PC, running the Windows 98 operating system, ran the instructor operator station (IOS) software, FSJ2000. This PC allowed the flight instructor to set up, control, and monitor the simulation. A separate Windows batch file (FSTEST.bat) could also be run to set up a direct console connection to the transputer system for troubleshooting and calibration purposes. The other PC ran the Windows NT operating system, and served as an image generator. Each PC was connected to a transputer card by an RS-422 serial data link. Both the IOS and visual generation software were proprietary, and backups were not available. The visual generator PC was originally equipped with a Diamond Fire GL 4000 graphics card, although this card had since been replaced with an NVIDIA card of undetermined type by the time 
the simulator arrived at the ADL.

The visual generator was connected to a single Epson LCD projector with a resolution of $800 \times 600$ pixels. A single flat projection screen was mounted in front of the simulator cabin; however, this screen was not present when the device arrived at the ADL. Alternately, the Vector documentation specified that a 60-in. big-screen television could be used instead of a projector. Due to the limited field of view provided by the projector, the cockpit windows were carefully shrouded, making them much smaller than the windows in an actual Cessna 172. Side windows were not provided. Provision was made to allow the pilot to rotate the simulated exterior view through $\pm 90^{\circ}$ using a knob mounted on the instrument panel. According to WWFC, this feature was very confusing, as its use resulted in side views being displayed through the front windows. Examples of the original visuals are shown in Figure 2.4.

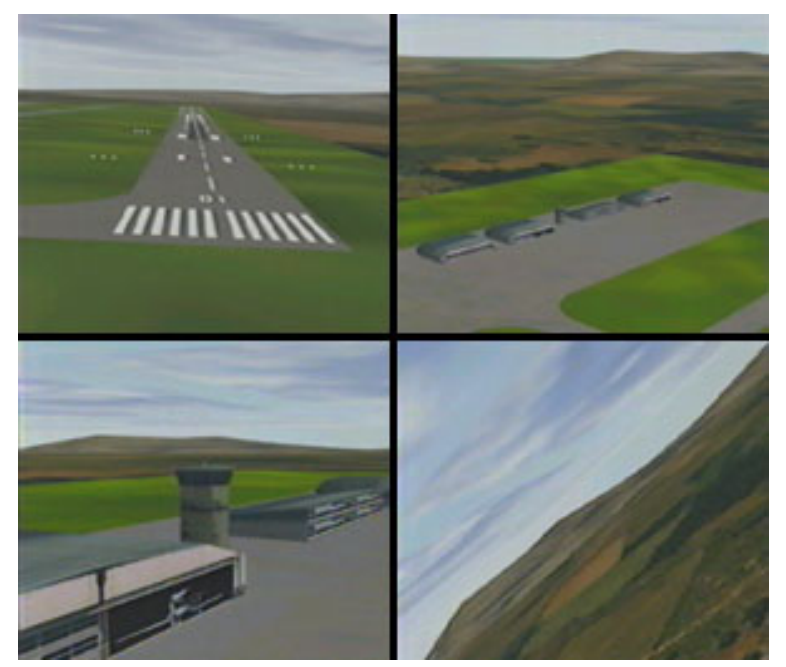

Figure 2.4: Typical visuals produced by the original image generation computer. Image by VTS courtesy of Archive.org [20].

The cockpit was equipped with a full set of round flight instruments (sometimes referred to in the aviation world as "steam gauges" for their superficial similarity to the gauges formerly found on steam engines), simulated radio equipment, and an actual Bendix-King KLN-89B GPS. The original cockpit is shown in Figure 2.5. 


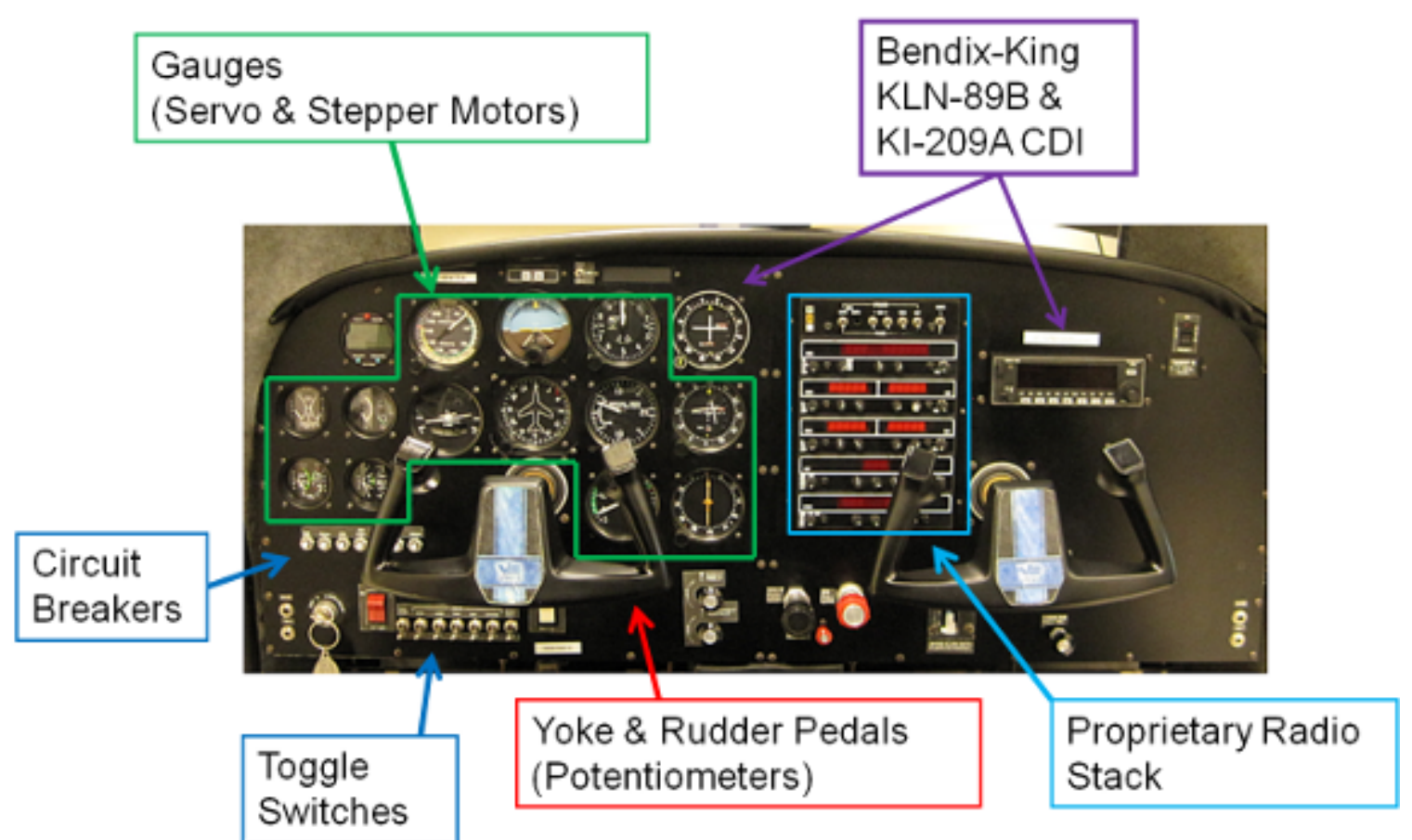

Figure 2.5: The original, unmodified cockpit of the VectorSim.

The majority of the flight instruments were simulations of the real units; however, two - the course deviation indicator and digital clock - were actual aircraft equipment. For the simulated instruments, most were driven by standard Futababrand servomotors, such as are commonly used on remote control model aircraft. Unless specially designed, however, hobby servomotors are not capable of performing complete $360^{\circ}$ rotations. For those instruments which required unbounded rotation, stepper motors were used in conjunction with position feedback provided by a photodetector and slotted-disc arrangement. The instrumentation originally installed in the VectorSim is itemized in Table 2.1. In total, there were 18 servomotors and 4 stepper motors divided between 14 simulated mechanical gauges.

Actual aircraft avionics used in the original VectorSim are listed in Table 2.3. These components are referred to as original equipment manufacturer (OEM) avionics to distinguish them from visually-similar simulated units such as the radio stack. OEM avionics are intended for use in actual aircraft. Simulated avionics are replica 
Table 2.1: Original VectorSim simulated instruments.

\begin{tabular}{|l|l|}
\hline Item & Underlying Technology \\
\hline Airspeed Indicator & Servomotor \\
\hline Attitude Indicator & Servomotor \\
\hline Turn Coordinator & Servomotor \\
\hline Vertical Speed Indicator & Servomotor \\
\hline Tachometer & Servomotor \\
\hline Course Deviation Indicator 2 (CDI 2) & Servomotor \\
\hline Fuel Quantity & Servomotor \\
\hline Fuel Flow / Exhaust Gas Temperature (EGT) & Servomotor \\
\hline Oil Pressure / Oil Temperature & Servomotor \\
\hline Vacuum / Amperage & Servomotor \\
\hline Altimeter & Stepper Motor \\
\hline Directional Gyro & Stepper Motor \\
\hline Automatic Direction Finder (ADF) & Stepper Motor \\
\hline Magnetic Compass & Stepper Motor \\
\hline
\end{tabular}


units which closely duplicate the look and operation of actual OEM components, but cannot be used in actual aircraft. Simulated avionics are typically much less expensive than OEM avionics.

Table 2.3: VectorSim OEM avionics.

\begin{tabular}{|l||l|}
\hline Item & Manufacturer \\
\hline Global Positioning System (GPS) Unit & Bendix-King KLN-89B \\
\hline GPS Annunciation Control Unit & $\begin{array}{l}\text { Mid-Continent Instrument } \\
\text { Co. MD41-228 }\end{array}$ \\
\hline Course Deviation Indicator 1 (CDI 1) & Bendix-King KI-209A \\
\hline Digital Clock / Outside Air Temperature & DAVTRON M803 \\
\hline Cessna Annunciator & Heads-Up Technologies CSEWCA-01 \\
\hline
\end{tabular}

The VectorSim radio stack was a simulated unit originally manufactured by Luftfahrtstudio Jungwirth, and consisted of a number of individual modules shown in Table 2.5

Slide potentiometers were used to measure the positions of the yoke, rudder pedals, and other controls. Shielded cables were used to carry the analog signals produced by the pots to an analog input card in the VectorSim I/O rack. An example potentiometer is shown in Figure 5.2 
Table 2.5: Simulated modules contained in the VectorSim radio stack.

\begin{tabular}{|l||l|}
\hline Item & Function \\
\hline Audio Panel & $\begin{array}{l}\text { Allows pilot to select which radio equip- } \\
\text { ment plays through the headset. }\end{array}$ \\
\hline Distance Measuring Equipment (DME) & $\begin{array}{l}\text { Displays distance to the currently- } \\
\text { selected DME beacon. }\end{array}$ \\
\hline NAV/COM Radio 1 & $\begin{array}{l}\text { Allows pilot to make and receive voice } \\
\text { transmissions and select navigational } \\
\text { radio beacons for CDI 1. }\end{array}$ \\
\hline NAV/COM Radio 2 & $\begin{array}{l}\text { Backup voice radio and navigational ra- } \\
\text { dio receiver for CDI 2. }\end{array}$ \\
\hline $\begin{array}{l}\text { Automatic Direction Finder (ADF) } \\
\text { Receiver }\end{array}$ & $\begin{array}{l}\text { Receives transmissions from the selected } \\
\text { non-directional beacon (NDB) or com- } \\
\text { mercial AM radio station, and indicates } \\
\text { the direction to the station on the ADF } \\
\text { instrument. }\end{array}$ \\
\hline Transponder (XPDR) & $\begin{array}{l}\text { Broadcasts aircraft identification num- } \\
\text { ber ("squawk code") and pressure alti- } \\
\text { tude to air traffic control services. }\end{array}$ \\
\hline
\end{tabular}




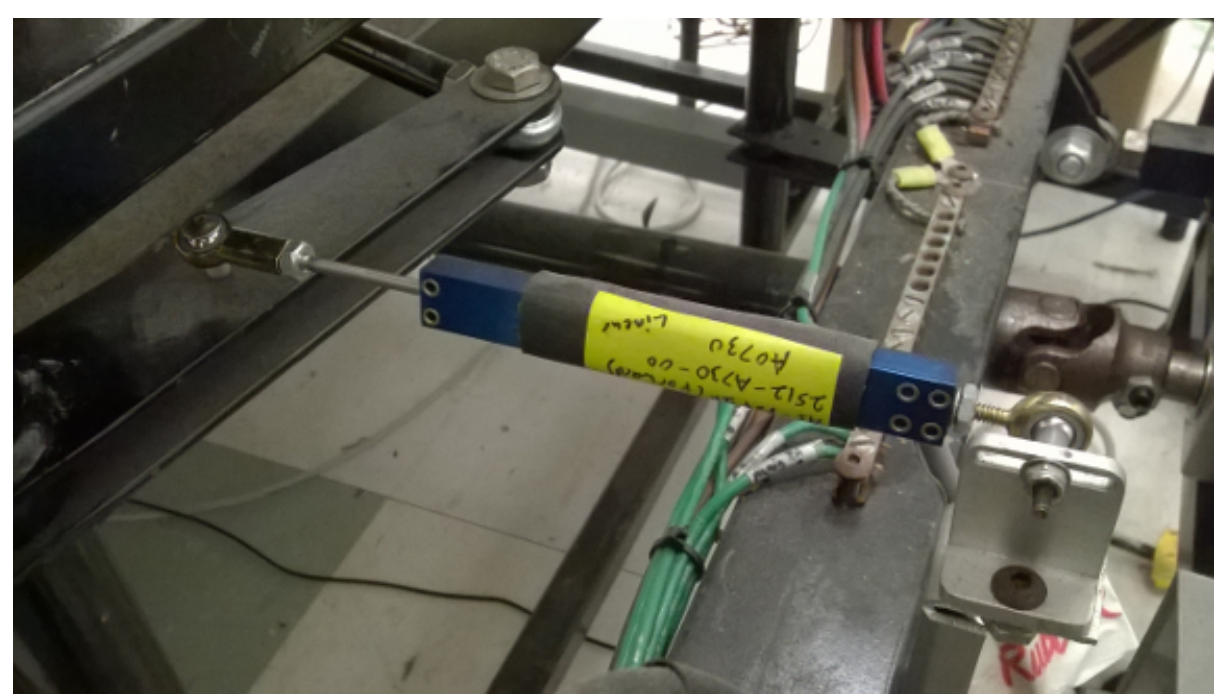

Figure 2.6: Slide potentiometers with rod ends were used for measuring the positions of the primary flight controls.

The Cessna 172 includes brake functionality as part of the rudder pedals. The brakes are activated by pressing on the top portion of each pedal. This allows for differential braking in order to decrease the turn radius while taxiing on the ground. In the VectorSim, the toe brakes were connected via pushrods to flexible steel strips mounted above the pedals on the frame of the simulator. The steel strips were instrumented with what appeared to be strain gauges. As the sensors were enclosed in heat-shrink tubing, it was not possible to determine the exact sensor type. The brake sensors were found to be connected to operational amplifier (op-amp) circuits mounted in a plastic project box referred to as the brake box, or "BB," shown in Figure 2.7. The brake box served as a signal conditioner for the brake sensors, converting their output into the same voltage range as that used by the potentiometers. The sensors and brake box circuitry were found to be still functional, so the exact sensor type was not investigated further. 


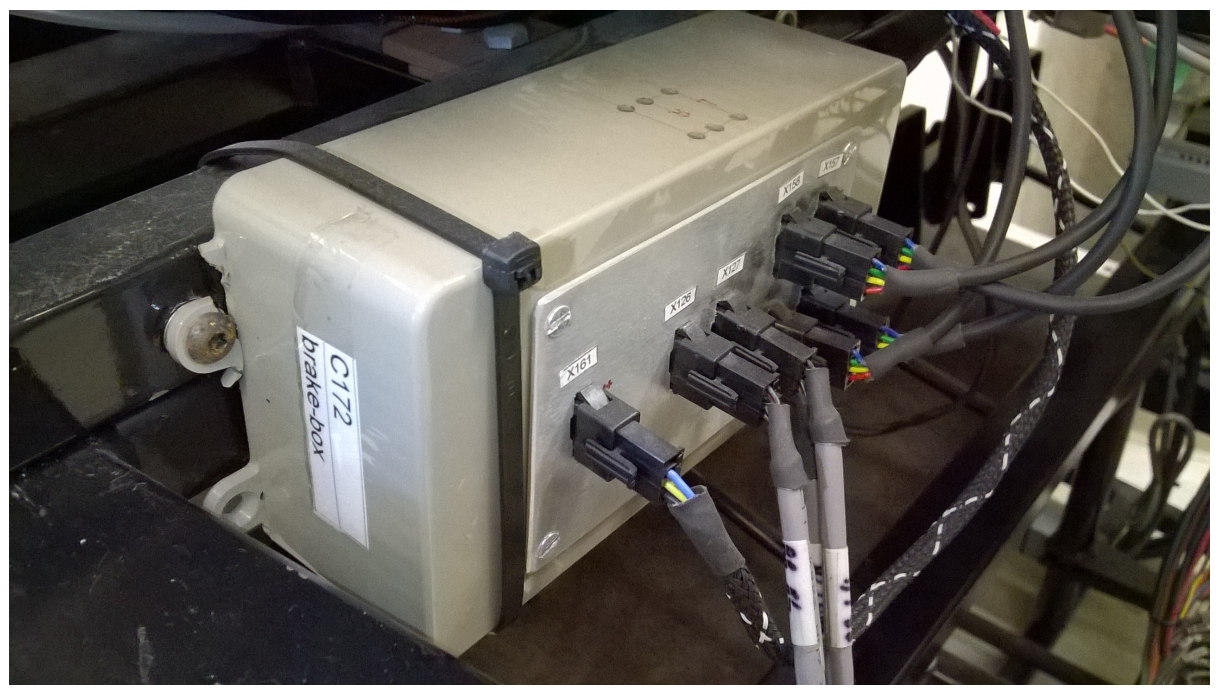

Figure 2.7: The brake box housed op-amp circuits for the toe-brake strain gauges.

Control loading on the pitch (elevator), roll (aileron), and yaw (rudder) axes was implemented using three Thomson OMNIDRIVE brushless servomotors and associated drive units mounted at the rear of the simulator cockpit. This equipment is shown in Figure 2.8. A system of pushrods and torque tubes installed beneath the cockpit floor connected the motors to the yoke and rudder pedals at the front of the cockpit. No force sensors (such as load cells) were included in the original control loading system. A modular terminal strip at the front of the simulator beneath the transputer I/O box served as the connection point between the motor drives and the transputer. This terminal strip is shown in Figure 2.9 


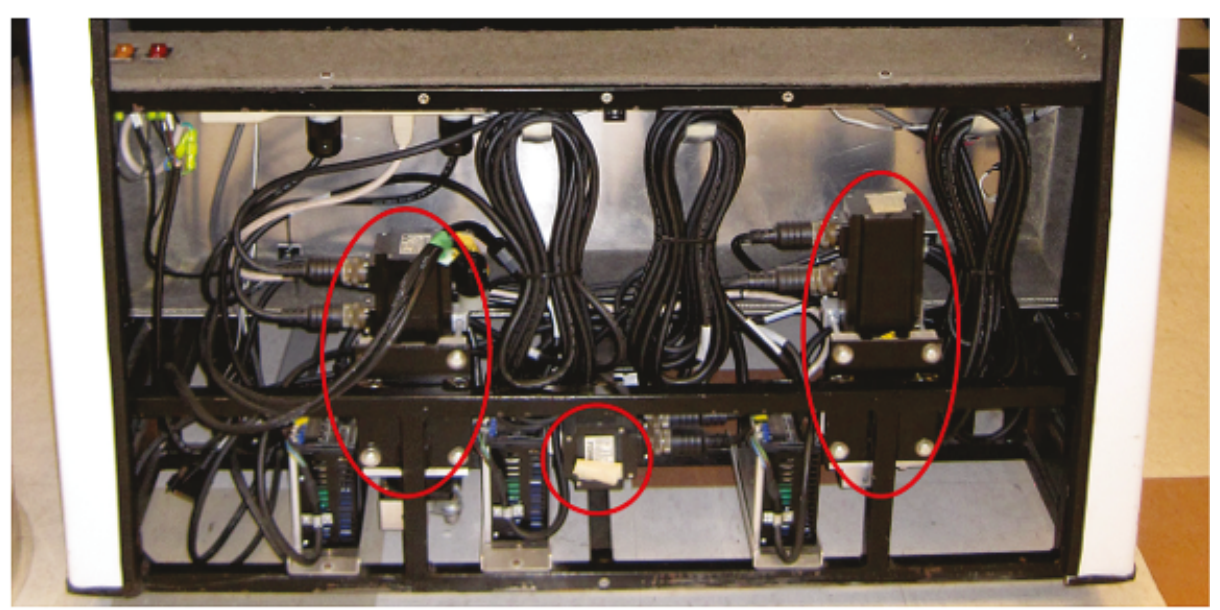

Figure 2.8: The control loading motors (circled) and associated drive units were mounted at the back of the simulator.

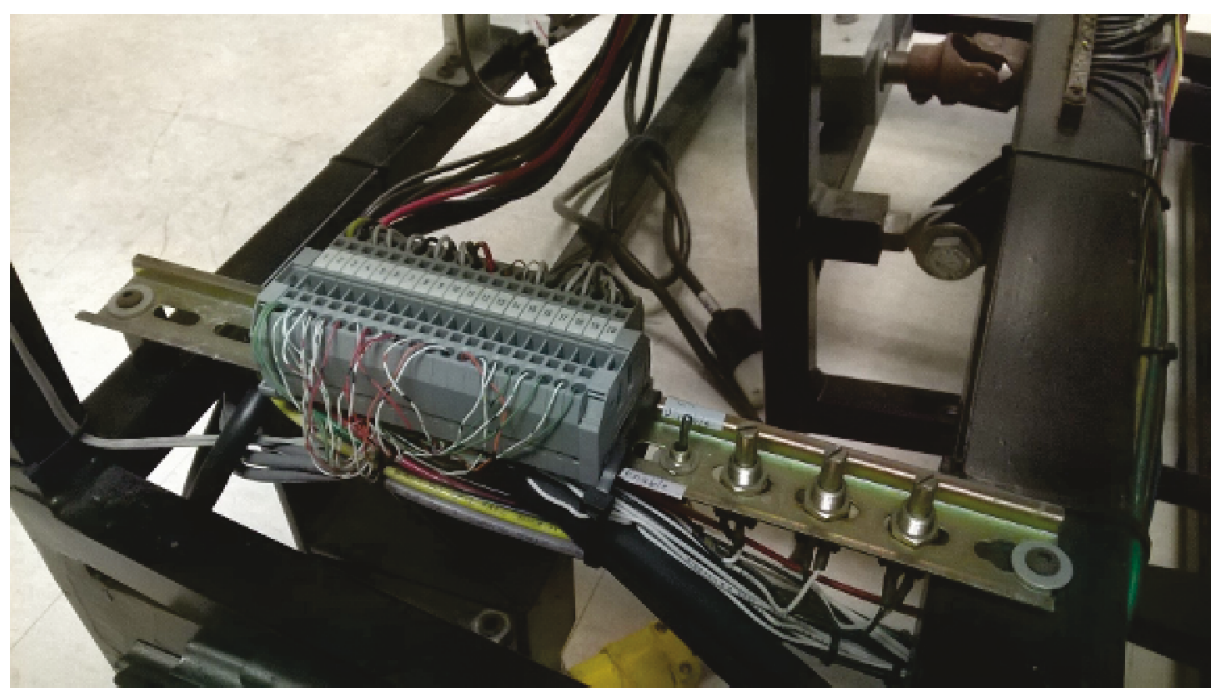

Figure 2.9: The control loading terminal strip facilitated connections to the control terminals on the brushless servomotor drive units at the back of the VectorSim.

Electric trim was provided by a $12 \mathrm{~V}$ DC electric motor connected via a friction clutch and system of cogs and bicycle chain to the trim wheel. The clutch allows the trim wheel to be rotated manually by the pilot if desired without also turning the electric motor and associated gear train. In some flight training devices, such as the Precision Flight Controls (PFC) Modular Flight Deck (MFD) currently in use at WWFC, the trim wheel is directly connected to the electric drive system, such that 
one may not be rotated without the other. In the author's experience, this design reduces realism both haptically and aurally compared with an actual aircraft. The electric trim motor in the VectorSim was activated by a switch on the pilot's yoke, as is the case in an actual Cessna 172. In an actual aircraft, the trim wheel moves a trim tab on the trailing edge of the elevator. The trim tab and other control surfaces on the Cessna 172 are shown in Figure 2.10. The trim tab deflects the air travelling over the elevator, causing the elevator itself to deflect in the opposite direction. This sets the desired position of the elevator, eliminating the need for the pilot to continuously exert force on the yoke. Using trim, the aircraft can thus be set to fly at a desired pitch attitude "hands-off," i.e. without pilot input. This reduces pilot workload and muscle fatigue, and results in a smoother flight path.

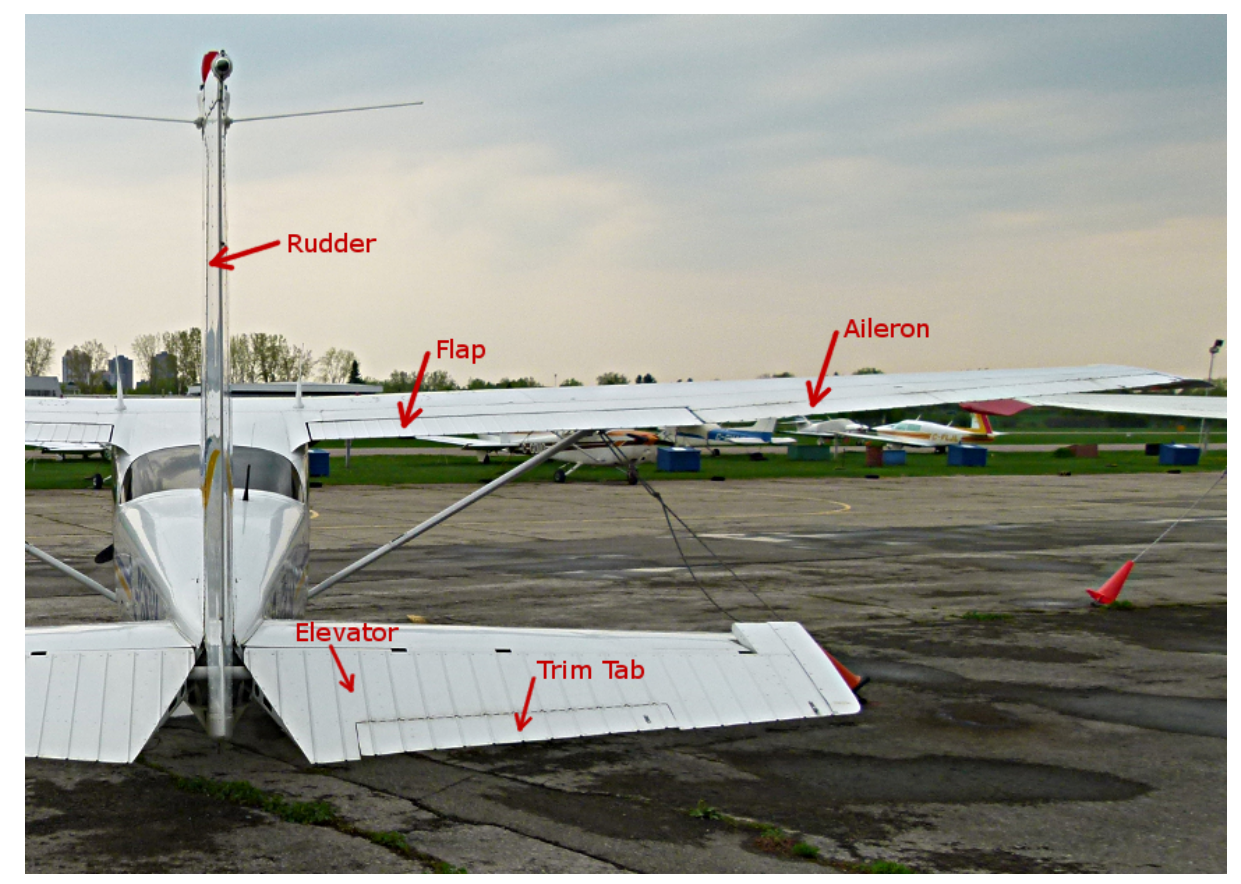

Figure 2.10: The control surfaces on a Cessna 172 aircraft.

A second $12 \mathrm{~V}$ DC motor and gear train system was used to move the pointer for the flap indicator. Flaps are high-lift devices mounted on the inboard trailing edge of the wing section on an actual Cessna 172, and may be deployed in increments $\left(10^{\circ}\right.$, 
$20^{\circ}$, or $30^{\circ}$ ) as desired, typically before landing.

A row of modular $12 \mathrm{~V}$ DC relays were used to enable various electrical systems within the simulator, such as the instrument lights. The relays may be seen in Figure 2.11a. Since computerized I/O equipment does not commonly operate at such high voltages, the relays were controlled via an intermediary board, referred to as the digital output interface (DO-IF) board, shown in Figure 2.11b. The DO-IF board contained a number of Darlington switches packaged as integrated circuits. A Darlington switch consists of two transistors, with the first turning on the second [21]. This allows the pair to switch a higher current than either device acting alone. In order to enable a given simulator system, transputer I/O cards would enable the appropriate Darlington switch, which would in turn apply 12 VDC to the coil of the corresponding relay. This caused the relay to close, enabling the connected system. This arrangement allowed the transputer to maintain control over the electrical systems, and disable them if required, for instance when simulating various equipment failures.

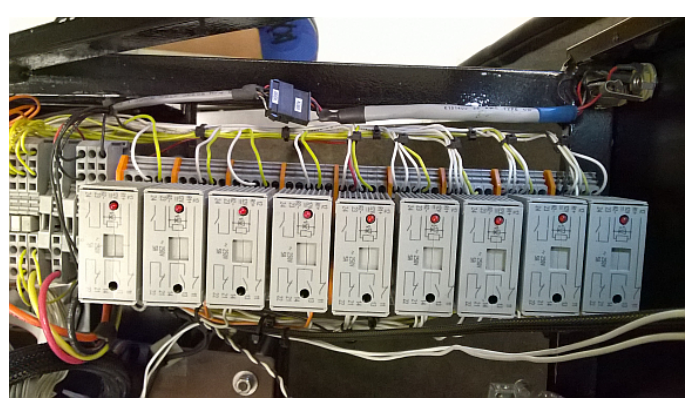

(a) Snap-on relays.

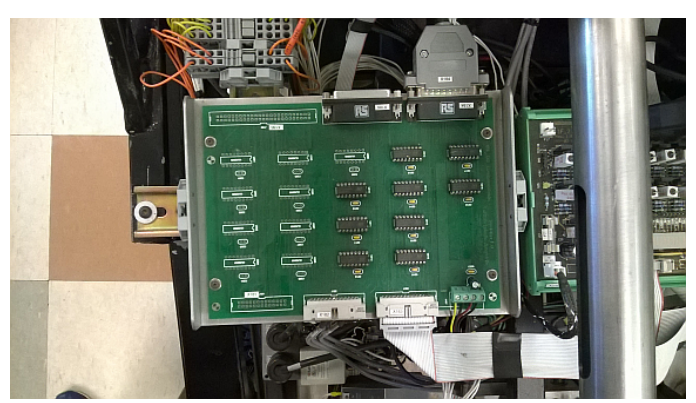

(b) Digital output interface (DO-IF) board.

Figure 2.11: The relay subsystem consisted of 12 VDC relays (a) activated by Darlington transistor switches (b).

A complete set of actual 0.5 A circuit breakers was also included in the VectorSim. Unlike the circuit breakers in a real aircraft, the breakers in the VectorSim served only to simulate the breakers which would be present in an actual Cessna 172. Their 
sole purpose was to trip on command from the transputer in the event of simulated electrical system failures, not to protect against over-current conditions. The breakers did not include dedicated sense terminals, so it was apparent that external circuitry was being used to detect the state of each breaker (tripped or untripped). This circuitry is discussed further in Chapter 5.

Two custom interface boards, also manufactured by Luftfahrtstudio Jungwirth, served to route and process signals between the transputer I/O rack and much of the cockpit hardware. These boards were known as KATIF-1 and KATIF-2, with the acronym standing for Katana Interface. This naming convention was due to the fact that the custom interface boards were originally intended to be used in a simulator for a Diamond Katana aircraft. The same technology was subsequently adapted to simulate the Cessna 172.

\section{$2.2 \quad$ First Power-up}

The original IOS and visual generator PCs were connected to the simulator and power was first applied on November 17, 2014. Both PCs were able to be booted to their respective legacy operating systems. The visual generation program appeared to hang without displaying any graphics. The IOS software started and permitted a user login using the supplied username and password. Logging in caused the gauges in the simulator cockpit to initialize, with both the servomotor and stepper-motor-based gauges rotating to their initial positions. It was also possible to select the desired scenery area within the IOS software, and view the corresponding map on-screen. It was not possible to actually start a simulation session, however, as the red aircraft icon which was supposed to indicate the current position on the map never appeared. After a few minutes, a communication error message would be displayed to the effect that the transputer could not be reached, despite the fact that the serial cable was 
connected. While power was applied, the Hobbs meter in the cockpit kept ticking, incrementing the total power-on time for the simulator. The LED indicators on the KATIF, transputer, and I/O cards also illuminated. The cooling fans also operated. In short, it appeared that much of the hardware in the simulator cockpit was still receiving power.

\subsection{Assessment and Decisions}

While much of the cockpit hardware was receiving power and was still operational, the IOS software was only partially-functional, while the visual generation software was totally non-functional, and was assumed to be corrupted. Since the IOS and visual generation software were custom products of the former Vector Training Systems, manufacturer support was not available. Re-installation media was also not available.

However, in the approximately decade-and-a-half since the original VectorSim was constructed, computers, graphics, and microelectronics technology has progressed significantly, while also decreasing in cost. Rather than attempt to troubleshoot and restore the legacy computer systems to their respective original states, it was decided that the simulator should instead be redeveloped, retaining as many components which were still functional, while upgrading and extending capabilities of the simulator wherever possible through the use of modern, cost-effective, commercial-off-the-shelf hardware and software products. 


\section{Chapter 3}

\section{Selection of Flight Simulation Software}

\subsection{Flight Simulation Theory}

The purpose of a flight simulator is take pilot input and transform it by means of a mathematical flight model into outputs which provide a reasonable approximation of the response of an actual aircraft. This process is shown schematically in Figure 3.1. As detailed in Chapter 1, full flight simulators provide the most accurate approximation (highest fidelity), while flight training devices typically provide a lesser level of fidelity, although at reduced cost.

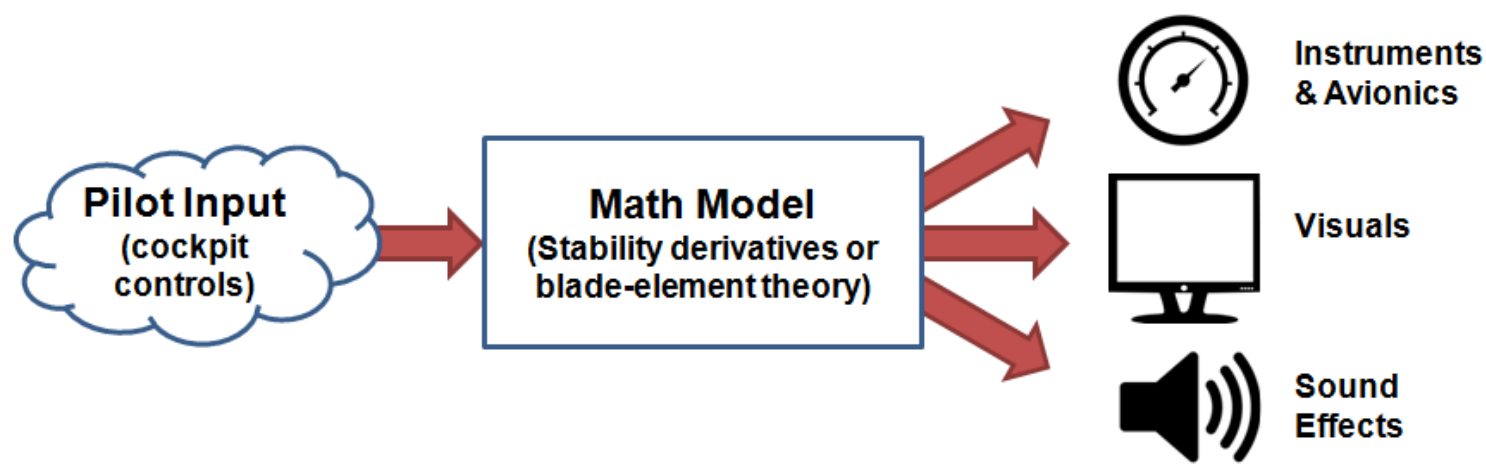

Figure 3.1: Flight simulation theory. Pilot inputs from the cockpit controls are processed by a mathematical flight model in order to generate appropriate outputs for the pilot.

There are three aspects of flight simulation. A mathematical model embodies the 
aircraft performance aspects of the flight simulator. Computer hardware provides a computational platform on which the mathematical model is run. Cockpit hardware provides the means by which the pilot interacts with the mathematical model. As a result, any simplifications included in the mathematical model will impact the realism of the flight simulator to some degree. However, flight simulation developers also recognize that an exact model is impractical, given the fact that the simulation needs to run in real time, and that simulation of certain aircraft behaviours outside the normal flight envelope do not always need to be modelled for training purposes. Effective mathematical simulation models that are suitable for training pilots in representative conditions strive for an appropriate balance between real-world fidelity and computational performance.

There are two main approaches used in the creation of mathematical models for the purposes of flight simulation:

\section{Stability Derivatives}

\section{Blade Element Theory}

The stability derivatives technique requires flight test or wind tunnel data for the aircraft being simulated [22-24]. Mathematically, stability derivatives are the partial derivatives of non-dimensional aircraft parameters with respect to other quantities in the simulation such as velocity, angle-of-attack, etc. For a given deviation from a steady, trimmed condition, the stability derivatives indicate the rate at which an aircraft will tend to return to the trimmed condition. The specific values of these derivatives are interpolated or extrapolated from lookup tables as required during the simulation. The equations required for the stability derivatives technique may be found in Swaine [10].

The blade element theory technique, on the other hand, is based on the physical 
shape of the aircraft, not on observed performance [22]. In this technique, the aircraft is divided into a discrete number of two-dimensional cross-sections, or elements. The aerodynamic forces on each element are calculated using the relative wind and aerodynamic coefficients specific to each element. The forces are then summed to determine the overall forces acting on the entire aircraft. Knowing aircraft mass, inertia, and other properties, the resulting accelerations are then determined.

Both of these techniques are used in flight simulation. The stability derivatives technique is generally more commonly used; however, it requires flight test or wind tunnel data, which is not always readily available. The blade element technique is well-suited to simulating situations where this data may be unavailable, such as for aircraft operating at the edge of their flight envelope, or untested experimental aircraft.

In choosing a suitable COTS flight model, as was an objective of this project, one must be aware of the extent to which the flight model affects the resulting simulation, but one must also rely to some extent on third party information, since in most cases mathematical flight models are proprietary and specific modelling details are not available.

\subsection{Flight Simulation Software Evaluation}

In order to be consistent with the COTS design philosophy and confine project scope to manageable levels, a pre-existing flight simulation software package was required. A number of potential software packages were evaluated, and are listed in Table 3.1.

The following criteria were kept in mind during the evaluation of each flight simulation software package:

- Graphics quality

- Instructor operator station (IOS) availability 
Table 3.1: Flight simulation software evaluated.

\begin{tabular}{|l|l|l|l|}
\hline Name & Developer & License & Website \\
\hline \hline Flight Simulator X & Microsoft & Proprietary & Unavailable \\
\hline Prepar3D & Lockheed Martin & Proprietary & www.prepar3d.com \\
\hline X-Plane & Laminar Research & Proprietary & www.x-plane.com \\
\hline FlightGear & Open-Source Community & GNU GPL & www.flightgear.org \\
\hline FixedWingLib & RTDynamics & Proprietary & www.rtdynamics.com \\
\hline FlightSIM & Presagis & Proprietary & www.presagis.com \\
\hline
\end{tabular}

- Availability of Cessna 172S model

- Cost/licence

- Ability to render multiple distinct views

- Input/output capability \& ease of interface

\subsubsection{Microsoft Flight Simulator $X$}

Microsoft Flight Simulator X (FSX) is no longer supported by Microsoft, and further development by the company has ceased [25]. (A downloadable version of the game has been re-released by Dovetail Games on the "Steam" online distribution service; however, an internet handshake is currently required at the beginning of each simulation session, thus ruling out the possibility of an offline simulator) $[26,27]$. While copies of the software are still available, it was thought unwise to base new development on an unsupported product. FSX was therefore eliminated from further consideration.

\subsubsection{Lockheed Martin Prepar3D Version 2}

Prepar3D (P3D) (pronounced "prepared"), is the continuation of Microsoft's professional flight simulation technology by Lockheed Martin as a training tool, as opposed 
to a game [28]. Since it is based on the same underlying technology, P3D is backwardcompatible with preexising add-on software originally intended for use with FSX.

\subsubsection{Laminar Research X-Plane 10 Global}

X-Plane is much more expensive than P3D for professional use; however, it offers several attractive benefits, such as native support for data input / output. X-Plane has also been used in several FAA-certified flight simulators [6, 29].

\subsubsection{Open-Source FlightGear}

FlightGear is an open-source flight simulator, featuring data input/output capabilities [30]. FlightGear has been used in the Canadian Civil Air Search and Rescue Association (CASARA) Virtual Trainer, a product of the Advanced Cognative Engi-

neering Lab at Carleton University [31,32]. One of the chief benefits of FlightGear is its native support for multi-channel graphics from the same instance of the software. For example, individual fields of view can be defined in a text file to create separate, geometrically-correct left, centre, and right views. However, while online documentation in the form of a wiki and basic manual are available, in-depth documentation suitable for developers wishing to interface to FlightGear was found to be incomplete and lacking in detail.

\subsubsection{Presagis FlightSIM}

FlightSIM is a standalone tool intended to allow the creation of high-fidelity flight and aircraft systems models without the need for programming on the part of the user. A 3D viewer application is included; however, this program is probably best used with dedicated image generation software, such as those available separately from Presagis. 


\subsubsection{RT Dynamics FixedWingLib}

FixedWingLib is a standalone flight dynamics model (FDM) intended for use in flight simulators and FTDs [33]. Since a FDM only calculates the physics of an aircraft, an external program is required to act as an image generator.

\subsection{Pros and Cons of Evaluated Software}

The benefits and drawbacks of each of the aforementioned flight simulation software packages were compared and are shown in Table 3.2.

Table 3.2: Pros and cons of flight simulation software evaluated.

\begin{tabular}{|c|c|c|}
\hline Software & Pros & Cons \\
\hline \multirow{5}{*}{ 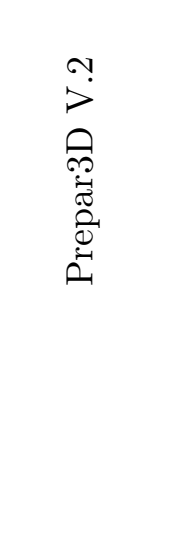 } & $\begin{array}{l}\text { Low-cost } \\
\text { (200 USD for commercial use). }\end{array}$ & $\begin{array}{l}\text { Multi-channel graphics not na- } \\
\text { tively supported (v.2) [34]. }\end{array}$ \\
\hline & Excellent graphics. & $\begin{array}{l}\text { Cessna } 172 \text { not included (third } \\
\text { party C172 available). }\end{array}$ \\
\hline & & IOS not included. \\
\hline & & GUI very simplistic and game-like. \\
\hline & & $\begin{array}{l}\text { Interfacing using native P3D SDK } \\
\text { or third-party FSUIPC program } \\
\text { appears complicated. }\end{array}$ \\
\hline \multirow{5}{*}{ 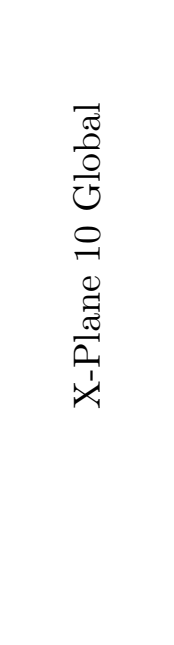 } & $\begin{array}{l}\text { Ability to extend functionality } \\
\text { with plugins. }\end{array}$ & $\begin{array}{l}\text { Incomplete documentation of plu- } \\
\text { gin SDK. }\end{array}$ \\
\hline & Excellent graphics. & $\begin{array}{l}\text { Poor documentation of UDP func- } \\
\text { tionality. }\end{array}$ \\
\hline & $\begin{array}{l}\text { Native data I/O using the UDP } \\
\text { network protocol. }\end{array}$ & $\begin{array}{l}\text { Expensive for professional use ( } 750 \\
\text { USD / licence). }\end{array}$ \\
\hline & $\begin{array}{l}\text { Native ability to log flight data to } \\
\text { file (for comparison with flight test } \\
\text { data). }\end{array}$ & $\begin{array}{l}\text { Curved-screen projection license is } \\
\text { expensive ( } 2000 \text { USD / license). }\end{array}$ \\
\hline & $\begin{array}{l}\text { Flight model based on blade ele- } \\
\text { ment theory. }\end{array}$ & \\
\hline
\end{tabular}




\begin{tabular}{|c|c|c|}
\hline & Cessna $172 S$ included by default. & \\
\hline & IOS included by default. & \\
\hline & $\begin{array}{l}\text { Large user-base, with multiple on- } \\
\text { line forums. }\end{array}$ & \\
\hline & $\begin{array}{l}\text { Previous experience with X-plane } \\
\text { at ADL. }\end{array}$ & \\
\hline \multirow{3}{*}{ 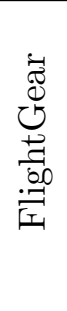 } & Open source (free software). & Limited in-depth documentation. \\
\hline & $\begin{array}{l}\text { Native multi-channel graphics sup- } \\
\text { port via XML file. }\end{array}$ & IOS not available. \\
\hline & Many data I/O formats supported. & $\begin{array}{l}\text { Cessna } 172 \mathrm{P} \text { included (as opposed } \\
\text { to S model). }\end{array}$ \\
\hline \multirow{2}{*}{ 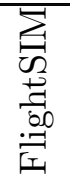 } & $\begin{array}{l}\text { Free for academic research (plus } \\
\text { yearly support fee). }\end{array}$ & Must build own aircraft models. \\
\hline & & IOS not available. \\
\hline \multirow{3}{*}{ 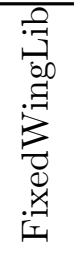 } & Customizable flight dynamics. & $\begin{array}{l}\text { Basic image-generation capabili- } \\
\text { ties. }\end{array}$ \\
\hline & Native flight data logging. & Must build own aircraft models. \\
\hline & & IOS not available. \\
\hline
\end{tabular}

\subsection{Final Software Selection}

In order to confine project scope to manageable levels, flight simulation packages which would have required substantial software or flight model development to implement in CURVS were eliminated from further consideration. These software packages included open-source FlightGear, Presagis FlightSIM, and RT Dynamics FixedWingLib, all of which lacked IOS functionality and a Cessna 172S flight model. The remaining two software packages were then compared against each other as shown in Table 3.3.

Both Prepar3D and X-Plane offered comparable, high-quality graphics. While X-Plane included both an IOS and Cessna 172S model by default, these essential 
Table 3.3: Comparison of Prepar3D versus X-Plane.

\begin{tabular}{|l||l|l|}
\hline Criteria & Prepar3D & X-Plane \\
\hline Graphics quality & High & High \\
\hline IOS included & No (3rd party available) & Yes \\
\hline Cessna 172S included & No (3rd party available) & Yes \\
\hline Cost & 200 USD/licence & 750 USD/licence \\
\hline Multi-view support & No & No \\
\hline Ease of interface & Hard & Medium \\
\hline
\end{tabular}

features were only available for Prepar3D at additional cost from third-party companies. However, this feature gap was offset by the lower cost per licence for Prepar3D compared with X-Plane.

Neither Prepar3D nor X-Plane support the generation of multiple distinct views from the same instance of the software, meaning that multiple copies of either program must be run on multiple networked computers in order to support a multi-projector cylindrical projection system.

Both Prepar3D and X-Plane support data input/output (I/O) with third party programs such as those required to interface with CURVS. In the case of Prepar3D, data I/O is accomplished by means of either the third-party FSUIPC (Flight Simulator Universal Inter-Process Communication) program by Pete Dowson or the native SimConnect SDK $[35,36]$. In the case of X-Plane, data I/O is accomplished by means of the either the native plugin SDK or native UDP interface [37].

Given similar functionality of X-Plane and Prepar3D, the final choice between these two software packages was based upon the perceived superior ease of interface provided by X-Plane, taking into account the prior software development experience of the author and the time available in which to implement a solution. Thus, X-Plane 10 Global was selected to replace the obsolete flight simulation and IOS software used 
in the original VectorSim.

\subsection{Software Interface to X-Plane}

This section provides a detailed overview of the specific mechanisms by which data may be exchanged with the X-Plane software. X-Plane allows data input/output to be performed in two ways:

1. Using a plugin run from within X-Plane;

2. Using the user datagram protocol (UDP) network interface for:

- Output of pre-defined data sets; and

- Input/output using predefined UDP commands.

The X-Plane plugin SDK is an officially-sanctioned third-party freeware project which enables the creation of plugins to interact with X-Plane [38]. A plugin is a pre-compiled piece of code which is run once each simulation cycle by X-Plane. An X-Plane plugin is in fact a "dynamic-link library" (DLL), renamed with the extension ".xpl" instead of ".dll," and placed in a specific location within the XPlane installation directory. Plugins can access internal X-Plane variables by means of handles known as "datarefs." Thus, plugins can be used to facilitate communication between external programs and X-Plane.

X-Plane also includes the ability to transmit and receive UDP datagrams over a local area network (LAN). UDP data output is straightforward to set up, as specific predefined datasets may be selected for output to a specified internet protocol (IP) address from within X-Plane. However, as each dataset contains up to eight individual variables, and the datasets are predefined, a large quantity of extraneous data may be transmitted along with the desired data. In addition, only a subset of the total number of X-Plane datarefs is available for direct UDP output. Furthermore, in 
almost all cases, the information available in the UDP datasets is representative of the actual state of the aircraft, not the indicated values displayed to the pilot in the cockpit. In the case of an instrument failure, for instance, the instrument in the cockpit will give an erroneous indication which differs from the actual value. Thus, the predefined datasets are not suitable for driving physical instruments in a flight simulator cockpit.

A third software interface to X-Plane exists, in which data may be passed to or requested from X-Plane by means of predefined command codes in UDP packets. Unfortunately, this native UDP interface to X-Plane is poorly-documented and proved difficult to understand and implement, despite the large amount of research time expended on it.

In the end, the open-source X-Plane Communications (XPC) Toolbox, released under an open-source licence by the NASA Ames Research Center, was selected for use with CURVS [39]. The toolbox consists of an X-Plane plugin, along with an SDK of predefined functions written in the $\mathrm{C}$ language. Functions for use with the Java, MATLAB, or Python scripting languages are also available. Instead of communicating directly with X-Plane, the XPC functions may be used by external programs to communicate with the XPC plugin. The XPC plugin then communicates with XPlane on behalf of the external program. Thus, the XPC toolbox greatly simplified the tasks of reading and writing X-Plane datarefs. 


\section{Chapter 4}

\section{Design of the Cockpit Interface System}

In order to control project cost and complexity, it had been decided that as much of the original cockpit hardware as possible would be reused in the redeveloped device. As detailed in Chapter 2, the flight controls, control loading motors, mechanical flight instruments, switches, annunciators, and cockpit lighting were for the most part all still functional. Thus, a robust yet cost-effective and customizable data acquisition (DAQ) and control system was required. The selected interface device(s) would thus serve as a bridge between the new flight simulation PCs and the existing cockpit hardware, as illustrated in Figure 4.1.

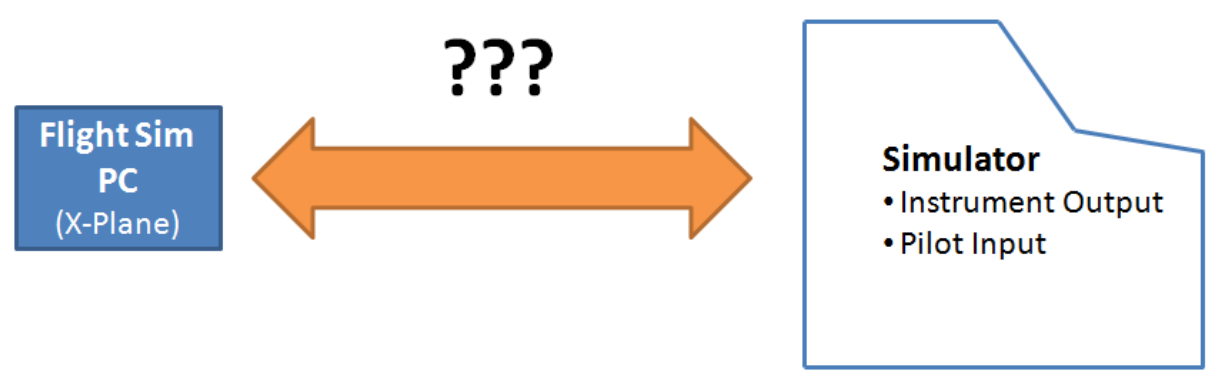

Figure 4.1: An interface device was required to bridge the gap between modern PCs and the existing VectorSim. 


\subsection{Interface Hardware Requirements}

Prior to selecting any interface devices to bridge the gap between the existing simulator cockpit and the new flight simulation computers, a set of requirements was compiled. These requirements are shown in Table 4.1

Table 4.1: Interface hardware requirements.

\begin{tabular}{|l|l|}
\hline Requirement & Reason \\
\hline $\begin{array}{l}\text { Fast processing speed ( }>\text { simulation } \\
\text { framerate) }\end{array}$ & I/O must occur with minimal latency \\
\hline Analogue input (13) & Required to read potentiometer voltages \\
\hline Digital input/output & Required for switches / relays \\
\hline Hobby servomotor control (18) & Servomotor-based simulated instruments \\
\hline Stepper motor control $(4)$ & Stepper-motor based simulated instruments \\
\hline Ethernet interface & Long runs of USB cable can be unreliable \\
\hline Cost-effectiveness & Project requirement \\
\hline
\end{tabular}

\subsection{Selection of Interface Hardware}

Given the wide range of different simulator components which needed to be either controlled or sampled from, and the fact that the relationship between specific cockpit inputs and simulated functionality was not always one-to-one, a programmable interface solution was required. For example, the authentic Cessna ignition key switch already installed in the cockpit had five positions, or states, but only three electrical terminals. For this device, it was found that the state of the switch (OFF, LEFT, RIGHT, BOTH, and START), was represented by the combination of terminals which were electrically HIGH at any given time. Thus, for this component, an interface device capable of computational logic was required to decode the switch state based on 
the states of the three terminals.

A number of potential interface devices were considered, and are shown in Table 4.3 .

Table 4.3: Potential interface devices.

\begin{tabular}{|l|l|}
\hline Device & Website \\
\hline National Instruments DAQ solutions & www.ni.com \\
\hline $\begin{array}{l}\text { Extended Programmable Input/output } \\
\text { Controller (EPIC) interface system }\end{array}$ & www.mindspring.com/ rrelect/ \\
\hline $\begin{array}{l}\text { Leo Bodnar universal USB interface } \\
\text { boards }\end{array}$ & www.leobodnar.com/ \\
\hline Phidgets I/O boards & www.phidgets.com/ \\
\hline Arduino microcontroller & www.arduino.cc \\
\hline Raspberry Pi single-board computer & www.raspberrypi.org/ \\
\hline $\begin{array}{l}\text { BeagleBoneBlack single-board com- } \\
\text { puter }\end{array}$ & http://beagleboard.org/bone \\
\hline LabJack DAQ solutions & https://labjack.com/ \\
\hline
\end{tabular}

Products from National Instruments were eliminated from consideration early on in the project, as the estimated cost of a complete interface system for all of the cockpit components exceeded the initial project budget at the time.

The Extended Programmable Input/output Controller (EPIC) system was also eliminated from consideration due to the fact that the available modules were not capable of controlling all of the necessary devices in the simulator (e.g., servomotor instruments), and available documentation was incomplete [40,41]. In addition, the user base appeared to be small, and active development limited.

Interface products from Leo Bodnar and Phidgets were all eliminated from consideration due to their exclusive use of the Universal Serial Bus (USB) $[42,43]$. The USB protocol uses unshielded cable and is therefore susceptible to electronic interference 
or "noise," especially when using long cables. Also, USB is limited to cable lengths of only $16 \mathrm{ft}$, which is a significant impediment for a simulator intended for commercial use [44]. Use of noise-susceptible communication cables was not permitted by the project requirements as laid out in section 1.4. (A workaround which allows use of the USB interface while eliminating long USB cables was later found, as detailed in Chapter 5).

The Arduino microcontroller platform also uses a USB connection by default; however, an add-on Ethernet daughterboard (or "shield") is available [45,46]. The Arduino (with USB) was successfully used as the I/O interface device on the KatanaSim, a prior simulation project at the ADL $[9,10,47]$. In addition, a pre-existing Ethernetenabled Arduino cockpit interface hobby project is available [48]. The Arduino platform has also been used for data acquisition from sensors on the Carleton University Simulator Project (CUSP), an innovative flight simulator currently under development as a fourth-year engineering project [49]. Compared to other options such as the Raspberry Pi or BeagleBone Black single-board computers, the Arduino microcontroller family runs much slower at $16 \mathrm{MHz}$ [45]. This was seen as a potential drawback, given that the total number of components to be interfaced to on the VectorSim greatly exceeded those of any of the aforementioned projects. Furthermore, users of the Arduino on the CUSP project reported reliability issues with the device, and thus it was thought best to seek a different solution, especially as CURVS will be subjected to heavy usage in an industrial setting, as opposed to a research lab. In professional flight training, the loss of a single day's worth of simulation time due to equipment malfunctions can result in hundreds of dollars in lost profit [8]. Thus, a more robust Ethernet-enabled interface solution was sought.

The Raspberry Pi single-board Linux computer is a popular, cost-effective platform for educational computing [50]. The Raspberry Pi 2 Model B, the latest available at the time, was evaluated for use on CURVS. The Pi costs approximately 40 USD, 
includes an Ethernet port, and 40 general-purpose input/output (GPIO) pins. However, as the $\mathrm{Pi}$ is intended to be used by the absolute beginner, in-depth information on programming the GPIOs using a compiled language such as $\mathrm{C}++$ is not readily available. Use of a compiled language (as opposed to interpreted scripting languages such as Python) was deemed essential in order to minimize latency in the redeveloped input/output system. For this reason, the Raspberry Pi was also eliminated from consideration.

The BeagleBone Black (BBB) is another single-board Linux computer [51]. At the time of comparison, the BBB (Rev. C) had a slightly faster processor (1 GHz vs. $900 \mathrm{Mhz}$, respectively) and the ability to use up to 69 programmable GPIO pins, significantly more than the Raspberry Pi [52]. The BBB also has 7 analogue inputs and an Ethernet port. In contrast to the $\mathrm{Pi}$, the $\mathrm{BBB}$ is aimed at a more technical user base. The BBB was also capable of driving all of the simulated flight instruments, when used in conjunction with external stepper and servomotor control boards by Pololu Corporation $[53,54]$. The BBB was also cost-effective, at approximately 59 USD per board. Thus, the BBB was initially selected as the base of the new interface system between the new, modern flight simulation PCs and the existing cockpit hardware, as illustrated in Figure 4.2.

\section{Single-board computer(s)}
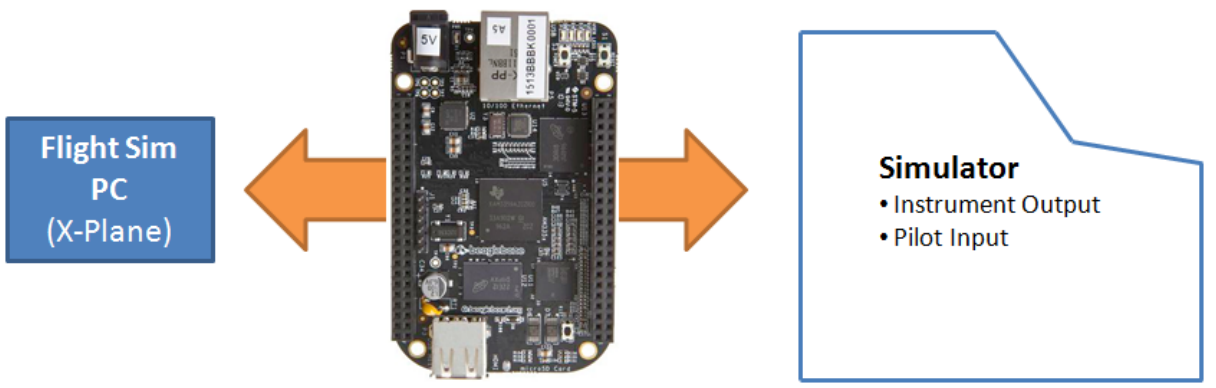

Figure 4.2: The BeagleBone Black single-board computer as an interface device. Image courtesy of the BeagleBoard.org Foundation.

One component of the VectorSim which required special attention was the existing 
control loading system. The brushless servomotors used in the system are powerful and can thus cause the flight controls to move quickly. For example, the maximum forces which can be output to the pilot at the controls ranges between about 20 lbs - 40 lbs. In fact, during testing of the control loading motors, it was found that the forces applied by the control linkages were sufficient to actually twist the chassis of the simulator slightly. As such, the control loading system was safety-critical. Unfortunately, the BBB System Reference Manual included a lengthy disclaimer which casts doubt on its suitability for use in safety-critical systems [52]. In addition, the $\mathrm{BBB}$ is incapable of generating the $\pm 10 \mathrm{VDC}$ signals required to control the brushless servomotor drives without external third-party modules. Thus, it was decided to seek a different, more robust method of interfacing to the servomotor drives in the control loading system. For this purpose the LabJack T7 DAQ was selected, along with two LabJack JLTick-DAC \pm 10 VDC analogue-output modules $[55,56]$. The LabJack is a professional-grade data acquisition and control device, yet was still cost-effective within the confines of the project budget at 399 USD for the DAQ, and 79 USD for each LJTick-DAC. The LabJack DAQ and LJTick-DAC modules are shown in Figure 4.3

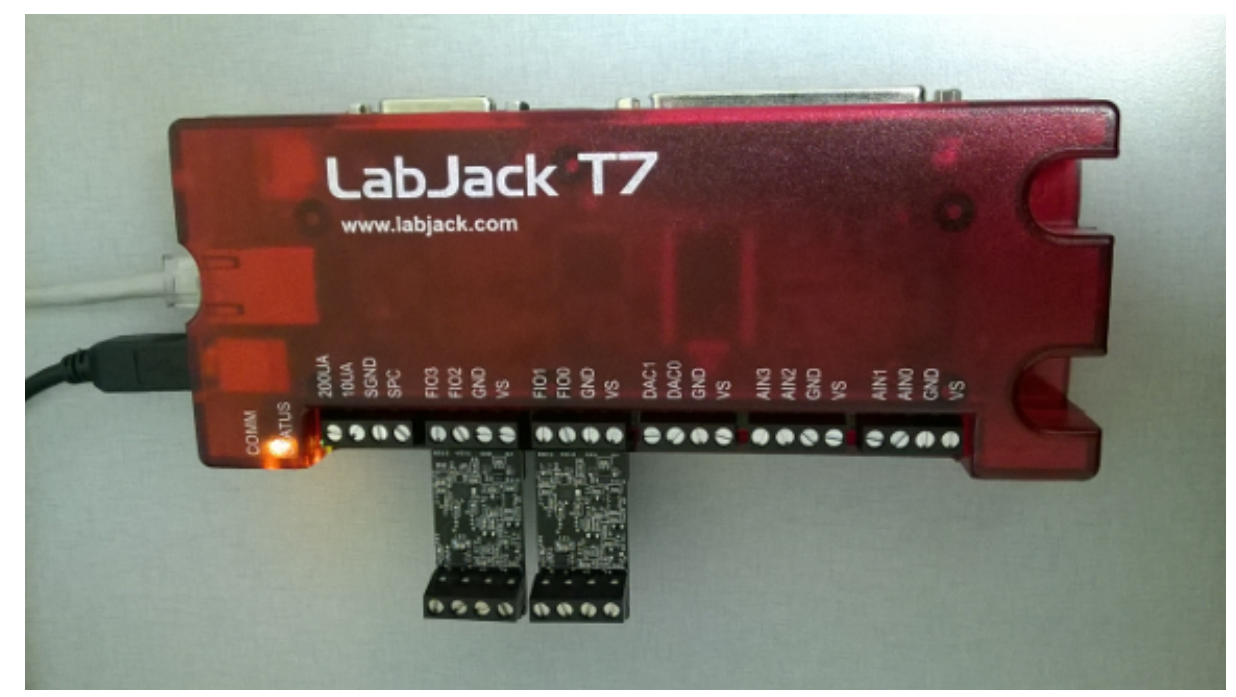

Figure 4.3: The LabJack T7 DAQ with two LJTick-DAC analogue output modules. 


\subsection{Initial Design of System Architecture}

Having selected the BeagleBone Black and LabJack DAQ as the main components of the redesigned cockpit interface system, a system diagram was created to show the method in which these components would be used. This diagram is shown in Figure 4.4. 


\section{Carleton University Redeveloped Vector Simulator (CURVS) System Architecture}

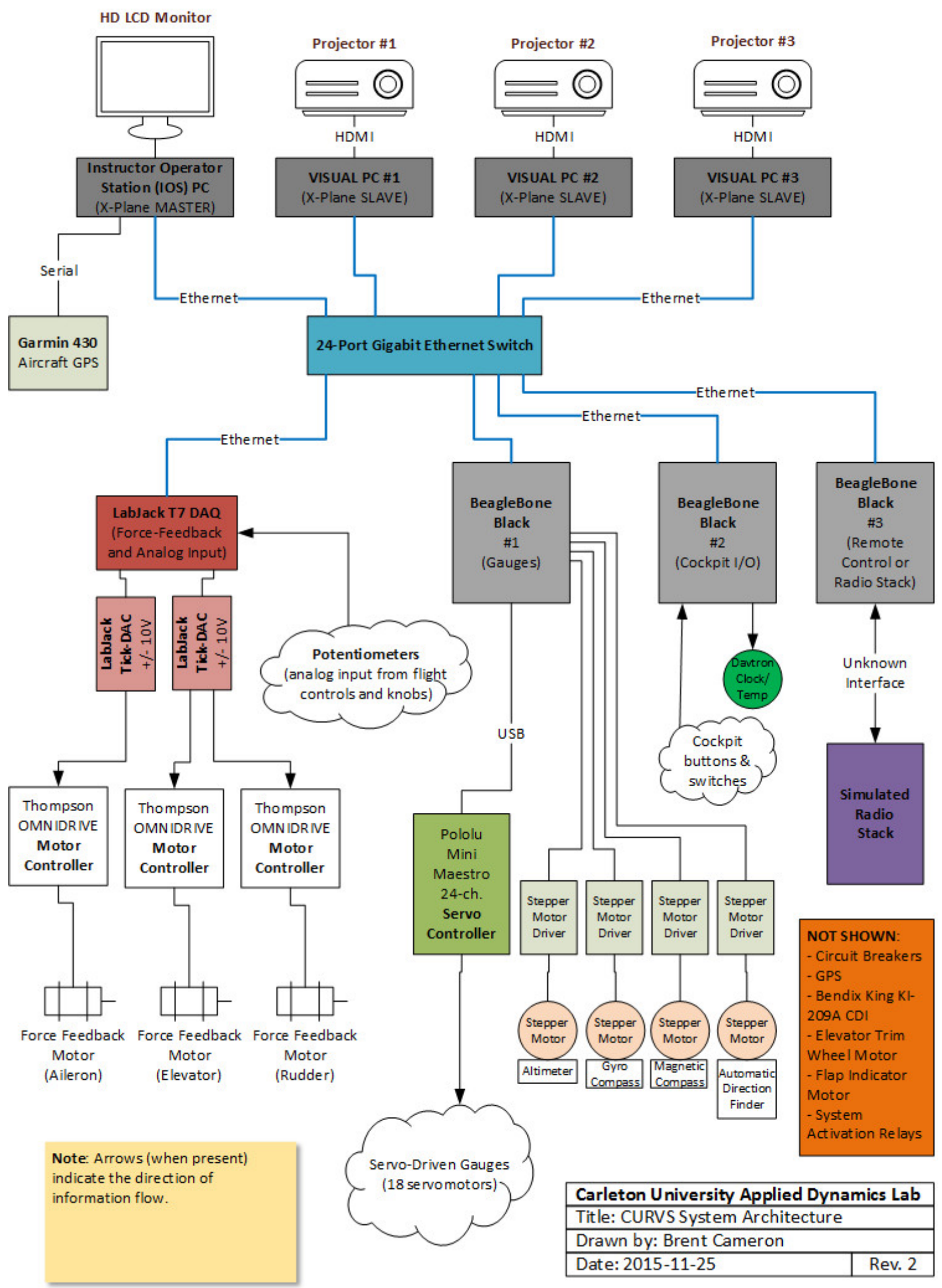

Figure 4.4: The initial design for the CURVS simulator interface system. 


\subsection{Revised Design of System Architecture}

As the project progressed, and development work progressed on programming the BBB, it became apparent that the BBB had several significant drawbacks which were not initially appreciated.

- Abrupt removal of supply power may cause corruption of the on-board solid state memory, or embedded multimedia card(eMMC) $[52,57]$. Thus, the BBB should always be shut down via either the Linux HALT software command or the power pushbutton on the board itself. In the context of CURVS, this issue would have reduced the robustness of the simulator while increasing its complexity by requiring the user to initiate a special shutdown sequence, with potential dire consequences if this step were to be forgotten.

- The GPIO pins on the BBB are only 3.3 V tolerant [52]. However, as reverseengineering progressed, it became apparent that much of the simulator ran on $5 \mathrm{~V}$ logic signals. Logic level shifters are commercially available; however, external custom circuitry would have had to be constructed. Use of custom circuitry was avoided wherever possible in order to comply with the COTS design philosophy.

- The analogue inputs on the BBB are only $1.8 \mathrm{~V}$ tolerant. However, the analogue signals in the VectorSim range from $0 \mathrm{~V}-5 \mathrm{~V}$. Voltage level shifting using op-amps or voltage divider circuits was a possibility; however, this would have required the construction of more custom circuitry and increased project complexity unnecessarily.

- The BBB processor will be damaged if power is applied to any of the GPIO pins if the BBB is in an un-powered state [52]. This limitation can be worked around, however; it also requires custom external circuitry. 
Given these shortcomings in the BBB platform, it was eventually decided to abandon the BBB in favour of a more robust, COTS solution. The solution chosen was to use LabJack T7 DAQs for the majority of cockpit interface tasks, not just for the control loading system. While much more expensive than the BBB (399 USD vs. 59 USD), the LabJack T7 has no special power-down requirements, is 5V-tolerant, and has no restrictions on the loading of any I/O pins before power-up. In addition, use of the LabJack T7 adhered to the COTS design philosophy as it required no external circuitry. In a commercial flight training environment, reliability is paramount, and time is money. Thus, the use of a more expensive yet more robust and reliable interface solution was justified. Furthermore, use of the same I/O hardware for as many interface tasks as possible reduced project complexity. This eliminated the need to program separate, incompatible interface systems, and allowed for the possibility of code reuse. The revised system diagram is shown in Figure 4.5. 


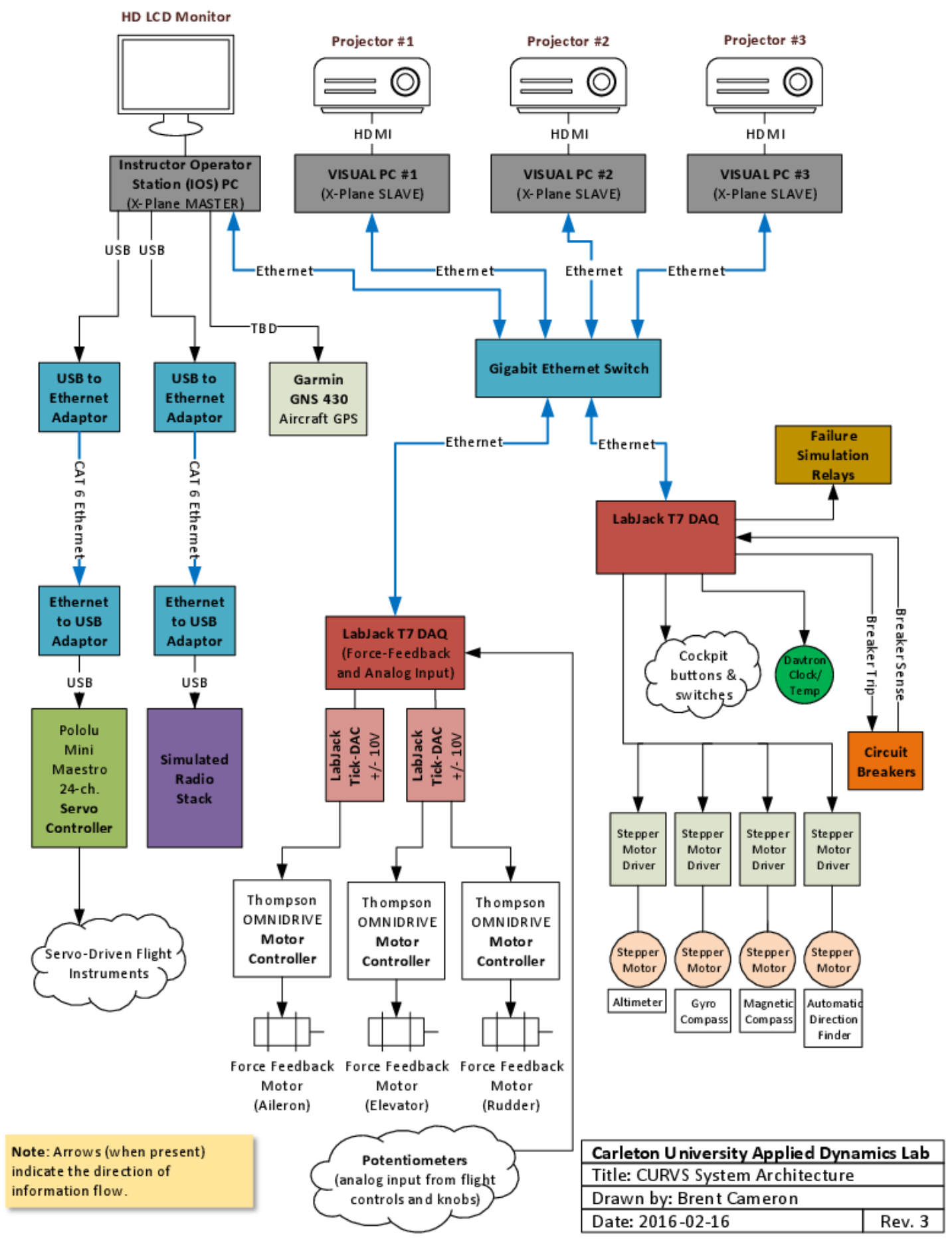

Figure 4.5: The revised design for the CURVS simulator interface system. 


\section{Chapter 5}

\section{Reverse-engineering and Redevelopment of Major Subsystems}

The existing hardware in the VectorSim was categorized into various subsystems based on function. First, each subsystem was reverse-engineered using the available documentation (if any), in order to determine how it was originally intended to work. Reverse-engineering is the process of analysing and understanding an existing system or device in order to duplicate or extend its functionality. This was often difficult due to the fact that the design documentation available was limited, and often differed from the VectorSim as built. As such, extensive manual wire-tracing and measurement with a multimeter or oscilloscope was often required, as shown in Figure 5.1.

Once a subsystem was reverse-engineered and understood, a method of interfacing with it was devised and physically implemented. Next, C\# or C++ program code was written in order to interface the subsystem to X-Plane 10, the new flight simulation program. Finally, the code was tested and debugged in the actual FTD. The process of reverse-engineering and interfacing the original VectorSim subsystems to modern flight simulation PCs and software resulted in the VectorSim being transformed into CURVS, the Carleton University Redeveloped Vector Simulator. This transition was in most cases accompanied by an increase in realism, performance, and fidelity, as 


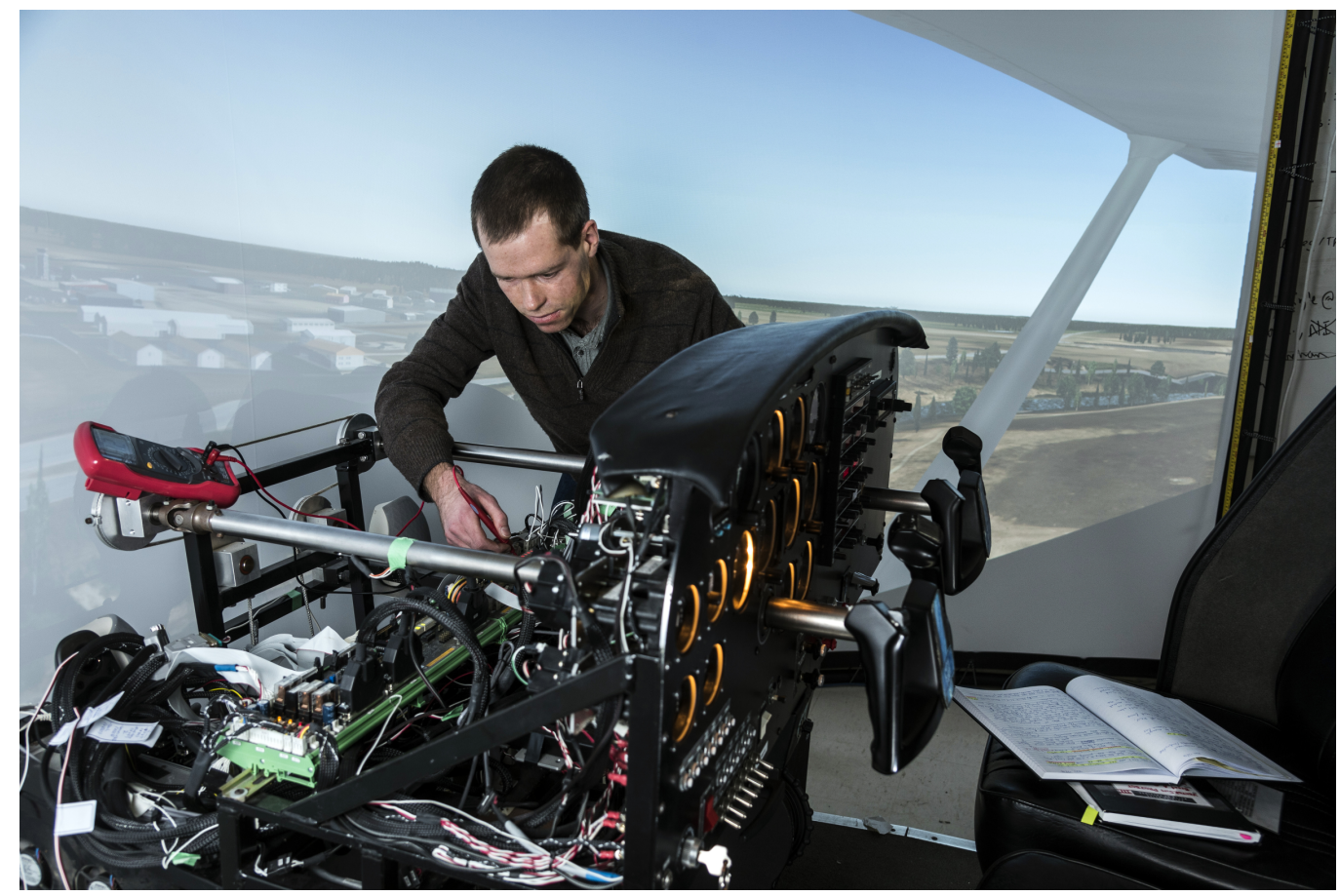

Figure 5.1: Due to limited documentation, much of the existing VectorSim hardware was reverse-engineered by hand. Image of the author by Carleton University.

the original VectorSim functionality was not only replicated, but modernized and extended, with new functionality being added.

\subsection{Available Documentation}

Approximately 100 pages of photocopied documentation consisting of copies of original wiring diagrams and hand-drawn sketches were obtained through the generosity of Jared Wilhelmi, Instructor of Simulator Maintenance Technology at Lake Region State College, in Devils Lake, North Dakota, United States of America. Lake Region State College still maintains two Piper Warrior FTDs by Vector Training Systems as part of their Simulator Maintenance Technician program [58,59]. Despite being simulations of a different aircraft, many similarities were found to exist with the VTS Cessna 172 FTD, as both types of FTD were in fact derived from a common 
design — a Diamond DA-20 Katana FTD. The history of this common design is detailed in Appendix A. Later on in the project, contact was also established with Jon Roloff, a former lead electronics technician at the now-defunct Vector Training Systems. Roloff kindly provided a large cache of original electronic documentation and transputer source code. The electronic documentation included a large number of wiring diagrams, and interconnect lists, some of which proved to be specific to the VTS Cessna 172. Unfortunately, the wiring diagrams were found to have been saved in the now-obsolete ".fh8" file format, once used by the legacy FreeHand 8 vector graphics drawing package by Macromedia, Inc. Although Macromedia has since been acquired by Adobe Systems, Inc., support for this file format in modern software such as Photoshop was found to be absent. Happily, a time-limited trial version of FreeHand 9 was discovered on an Internet freeware website and was used to open the original drawings. Foxit Reader PDF Printer from Foxit Software Inc. was then used to convert the original .fh8 files to the ubiquitous, modern Portable Document Format (PDF) [60]. The Roloff documents proved to be highly useful, and being in full colour and complete, were used to verify the guesswork which had sometimes been required previously when using the photocopied documents from Lake Region State College. As might be expected when working with legacy documentation, some differences between the FTD as built and the FTD as documented were also noticed.

\subsection{Analogue Input}

The analogue input subsystem on the VectorSim was responsible for sensing the positions of various controls in the cockpit, such as the throttle, yoke, and rudder pedals. This system consisted of thirteen analogue sensors connected to a custom analogue input card in the transputer I/O rack. The sensors were divided into 9 potentiometers and what appeared to be 4 strain gauges for measuring toe brake 
displacement. The potentiometers were wired as shown in Figure 5.2 and supplied with 5 VDC. The voltage between the movable wiper (represented as an arrow in Figure 5.2) and ground varies depending on wiper position, allowing a potentiometer to act as a displacement sensor.

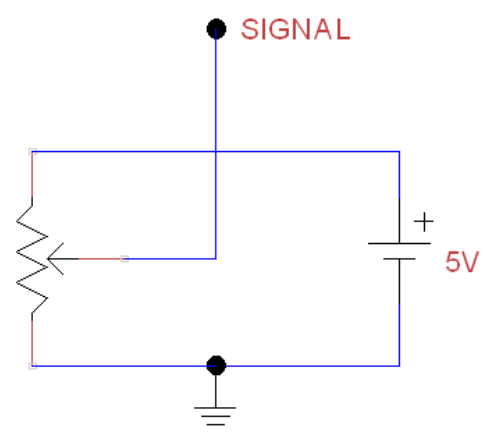

Figure 5.2: Connection of a potentiometer for use as a position sensor.

\subsubsection{Early Experiments on the Analogue Input Subsystem}

As funding was not immediately available, interface work commenced using whatever supplies and equipment were easily accessible or free. Flight controls and visuals are the most essential components of any flight simulation project, so initial work focused on restoring the functionality of these two systems. Potentiometers were already installed on the primary flight controls (aileron, elevator, and rudder), so it was decided to start by connecting to these three sensors directly, and interfacing them to a PC running a copy of FlightGear, a free, open-source flight simulation program [30]. Although this software was not ultimately selected for use in the final design for the reasons previously mentioned in Chapter 3, FlightGear proved to be an excellent learning and development tool.

In this preliminary research, the Arduino-compatible Teensy 2.0 microcontroller was used to successfully interface the selected potentiometers in the VectorSim to an 
external PC $[45,61]$. The required circuitry is illustrated schematically in Figure 5.3. The Teensy microcontroller is unique among Arduino-compatible microcontrollers in that it can be set up to natively emulate a standard USB joystick. This allows custom game controllers to be created with relative ease, as all interface code runs on the microcontroller itself, and no custom software is required on the host PC. Such a Teensy-enabled custom game controller is thus automatically compatible with any program which is able to use a USB joystick. The Arduino code which was run on the Teensy for the preliminary interfacing experiments is included in Appendix B. The Teensy microcontroller with associated interface circuitry is shown in Figure 5.4.

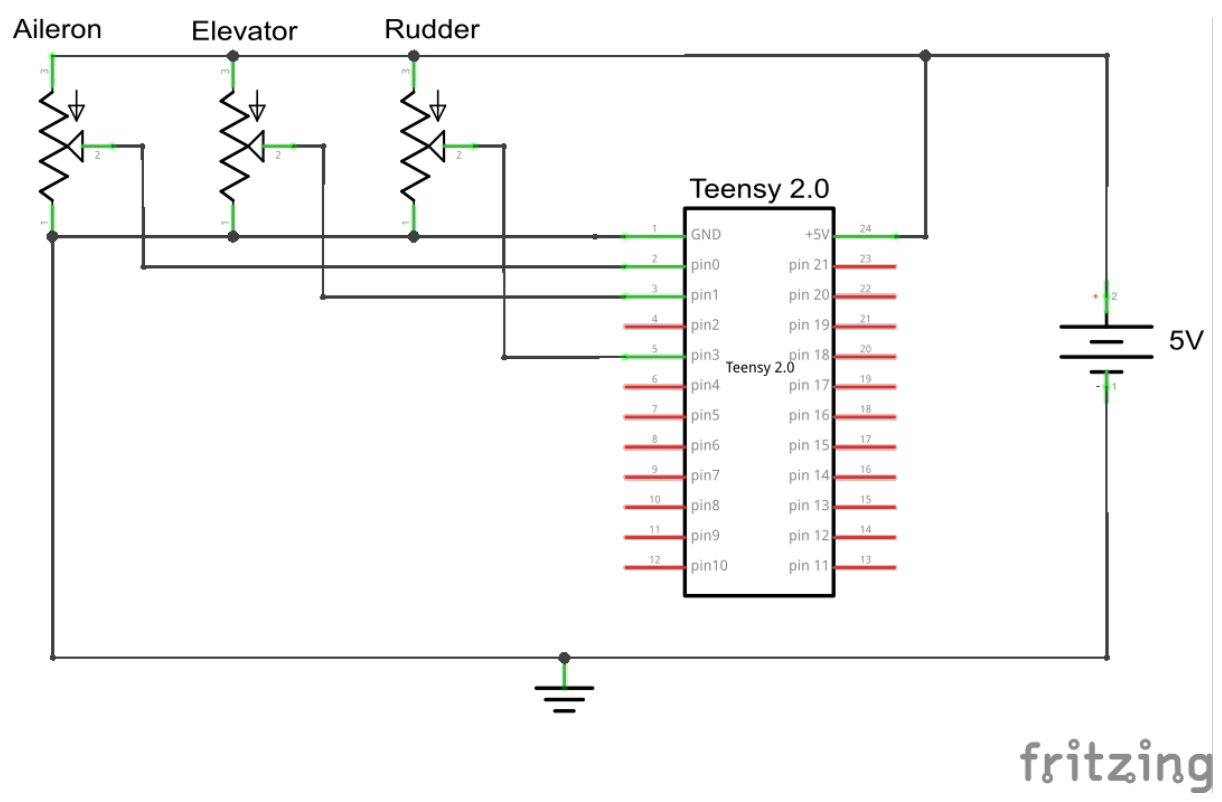

Figure 5.3: Teensy microcontroller interface schematic. Note that the USB connection to the simulation PC is not shown. 


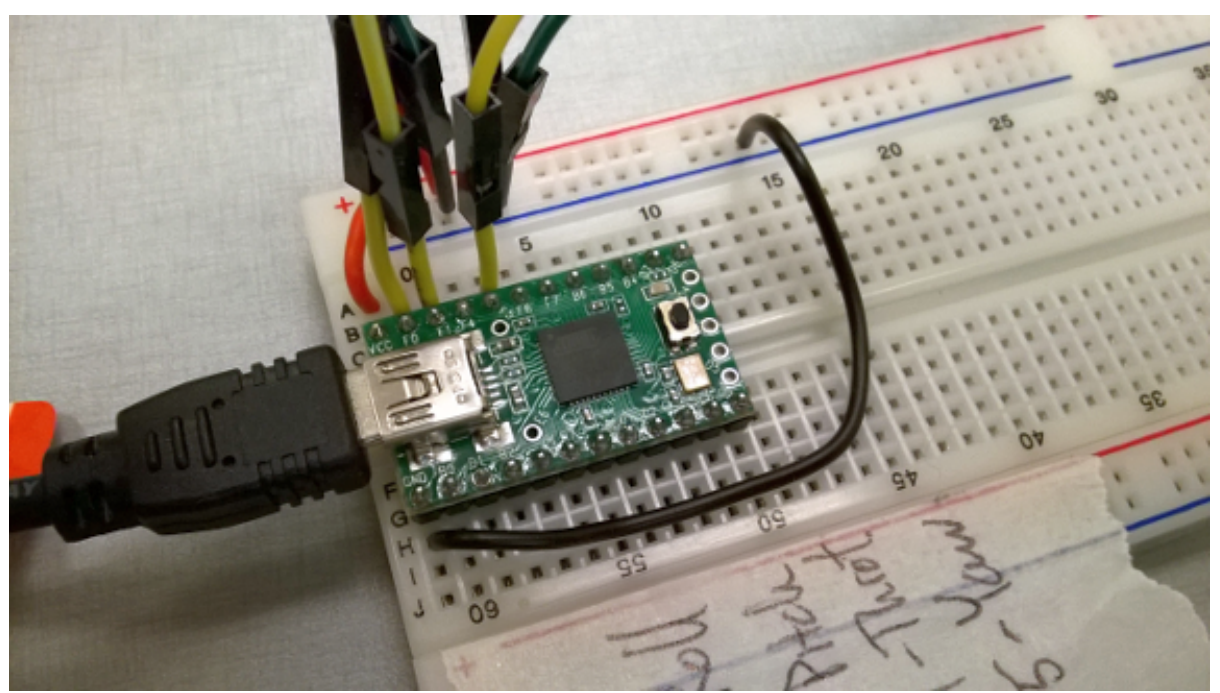

Figure 5.4: Teensy 2.0 microcontroller ready for connection to the flight control potentiometers. Note the size of the board compared to the mini USB connector.

A temporary visual system was implemented using three high-definition (HD) televisions mounted on a custom stand which was left over from a previous project at the ADL. The three HD televisions were driven from a single PC using a Matrox TripleHead2Go multi-display adapter [62]. The PC used to run FlightGear was a surplus unit which was obtained following upgrades to an undergraduate computing lab at Carleton University. This PC was equipped with an Intel i5 central processing unit (CPU), 4 GB of random-access memory (RAM), and 1 GB of dedicated video RAM on an NVIDIA 8900GT-based video card by ASUS. FlightGear was found to run smoothly on this system. This temporary setup allowed the VectorSim to be "flown" for the first time after approximately 7 years in storage, as shown in Figure 5.5 


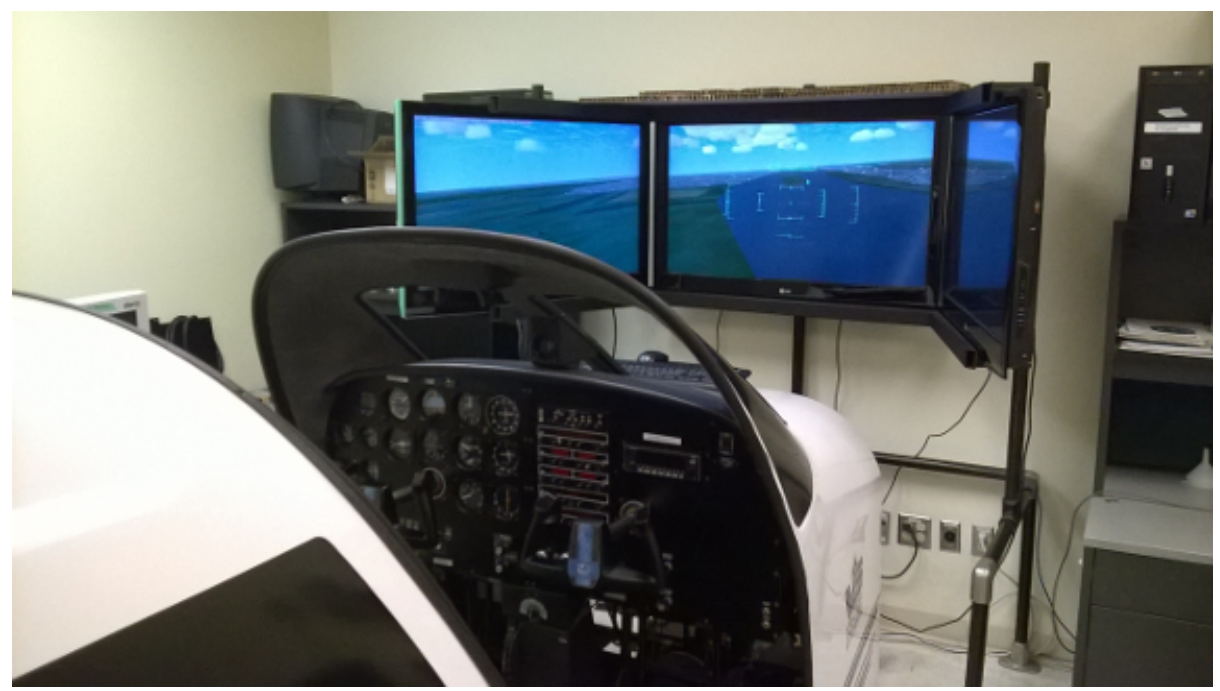

Figure 5.5: VectorSim with primary flight controls connected to the FlightGear software using a Teensy microcontroller configured as a USB joystick.

\subsubsection{Interfacing to the Existing Analogue Input Subsystem}

In the original VectorSim, 3-conductor, 24 AWG shielded cable was used between the analogue input card and all sensors. The drain wire of each cable was connected to the chassis of the simulator at the sensor end. The other end of each drain wire was not connected in order to prevent ground loops. A ground loop occurs when the two ends of a ground wire are at differing electrical potentials [63]. This causes an unintended current to flow on the ground wire.

The individual sensors in the analogue input subsystem, their purposes, specifications, and the LabJack analogue input (AIN) channels eventually used to interface to them are listed in Table 5.1 
Table 5.1: Analogue input sensors

\begin{tabular}{|l|l|l|l|}
\hline Name & Sensor Type & Nominal Value & LabJack AIN \\
\hline \hline Elevator & Slide pot & $1 \mathrm{k} \Omega$ & AIN0 \\
\hline Aileron & Slide pot & $1 \mathrm{k} \Omega$ & AIN1 \\
\hline Rudder & Slide pot & $1 \mathrm{k} \Omega$ & AIN2 \\
\hline Throttle & Slide pot & $1 \mathrm{k} \Omega$ & AIN3 \\
\hline Mixture & Slide pot & $1 \mathrm{k} \Omega$ & AIN4 \\
\hline Trim, Elevator & Rotary pot & $1 \mathrm{k} \Omega$ & AIN5 \\
\hline Flap Position Handle & Rotary pot & $100 \mathrm{k} \Omega$ & AIN6 \\
\hline Flap Position Indicator & Rotary pot & $100 \mathrm{k} \Omega$ & AIN7 \\
\hline QNH & Rotary pot & $1 \mathrm{k} \Omega$ & AIN8 \\
\hline \hline Brake Box, Pilot, Right (BBLR) & Strain gauge & N/A & AIN9 \\
\hline Brake Box, Pilot, Left (BBLL) & Strain gauge & N/A & AIN10 \\
\hline Brake Box, Copilot, Right (BBRR) & Strain gauge & N/A & AIN11 \\
\hline Brake Box, Copilot, Left (BBRL) & Strain gauge & N/A & AIN12 \\
\hline
\end{tabular}

The pinout of the analogue input cable on the VectorSim was reverse-engineered using a multimeter in continuity check mode with the assistance of Karim Youssef, an undergraduate engineering volunteer at the ADL. Once it was determined which pins on the DB-37 connector were connected to which potentiometers in the simulator, a custom patch cable was constructed to connect these potentiometers to the analogue inputs (AINs) on the LabJack T7 DAQ. The resulting custom analogue input patch cable is shown schematically in Figure 5.6, while an image of the actual cable while it was being built is shown in Figure 5.7. 


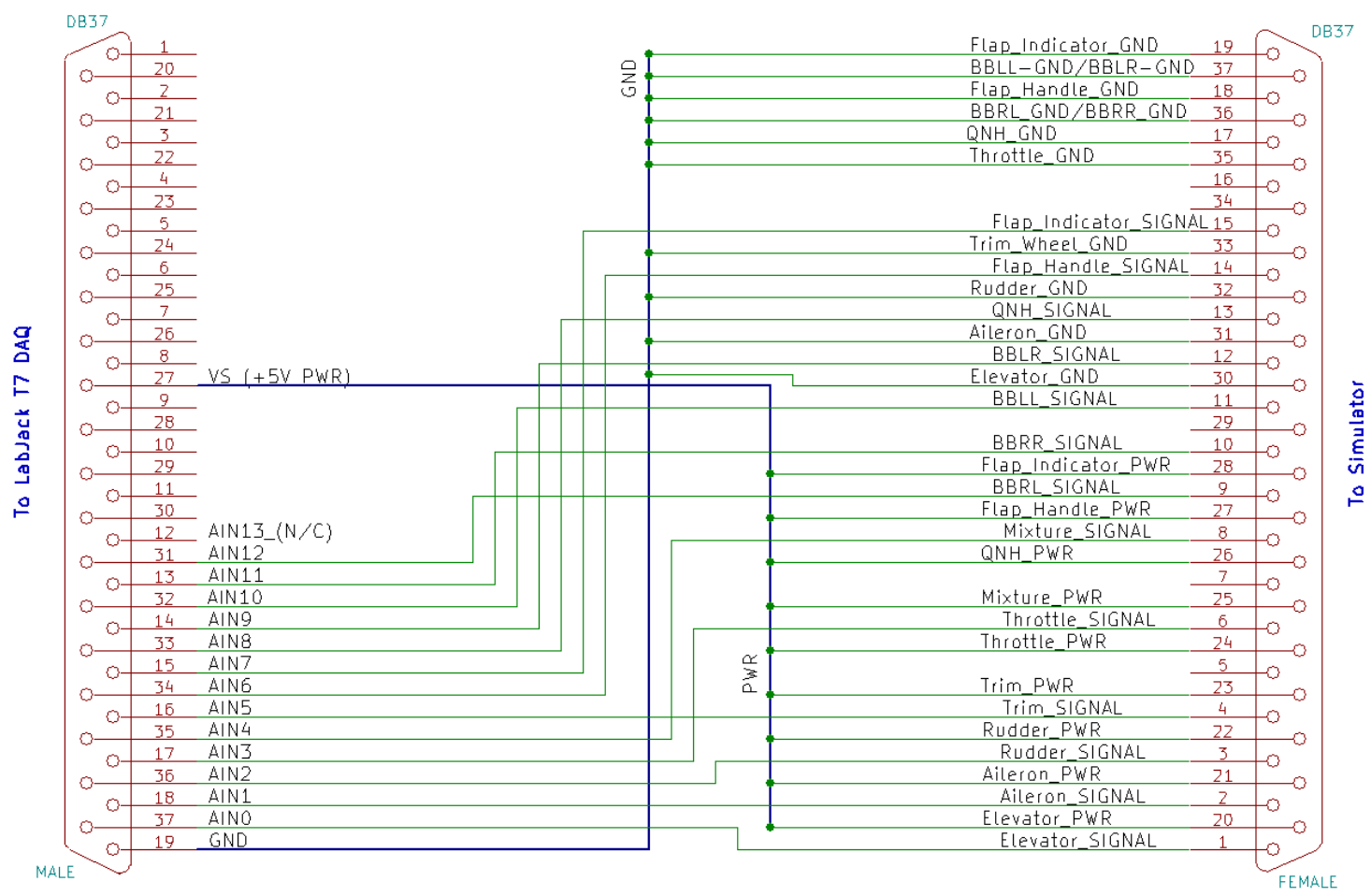

Figure 5.6: Custom analogue input patch cable to connect simulator to LabJack T7 DAQ.

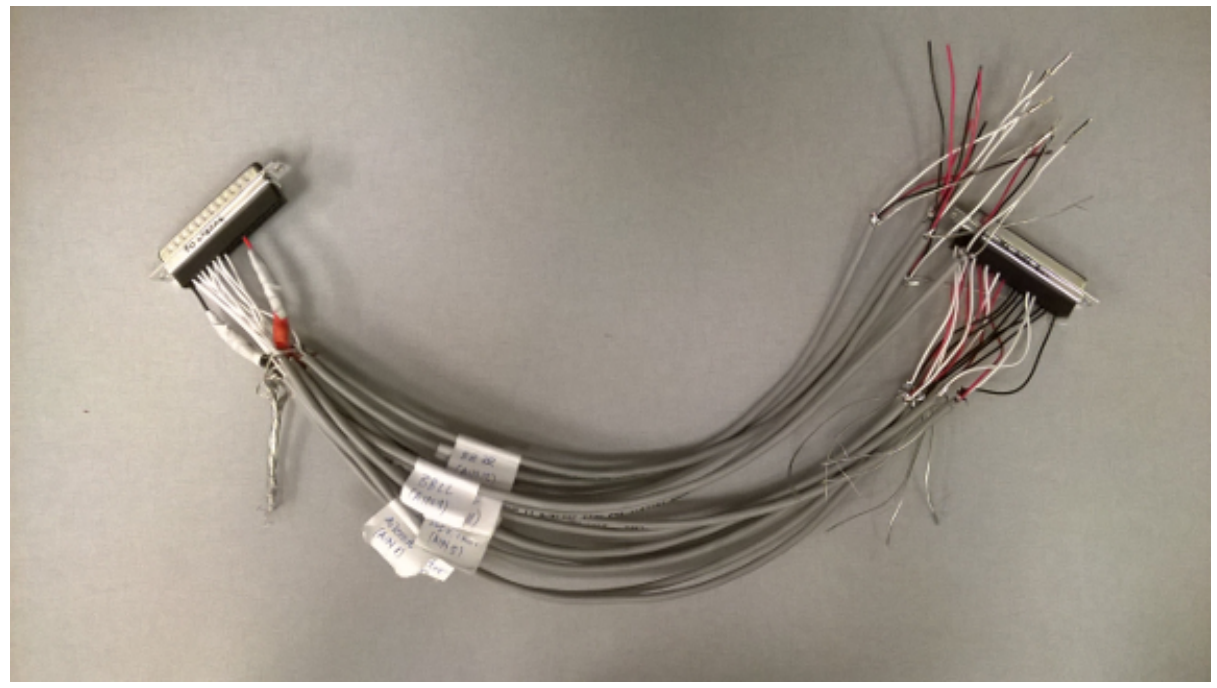

Figure 5.7: Custom analogue input patch cable in the process of being constructed. 


\subsubsection{Analogue Input Interface Program}

A physical interface cable and LabJack DAQ are useless without a software program to actually quantize the inputs and communicate the corresponding control positions to X-Plane. LabJack Corporation provides a well-documented software development kit $(\mathrm{SDK})$ for use with their products which is written in the $\mathrm{C}++$ programming language. Wrappers are available for a number of other languages; however, as $\mathrm{C}++$ is a compiled language (and therefore fast), and the author was most familiar with it, $\mathrm{C}++$ was selected as the language to be used for the LabJack analogue input interface program. Control loading functionality was later added to this program as well, in accordance with the revised system architecture for CURVS presented in Chapter 4. For simplicity, the LabJack analogue input interface program was written as a console program; i.e. without a graphical user interface (GUI), and intended to be run on the same PC as X-Plane. Communication with the LabJack occurs over the simulation LAN. Program flow within the analogue input interface program is shown in Figure 5.8. 


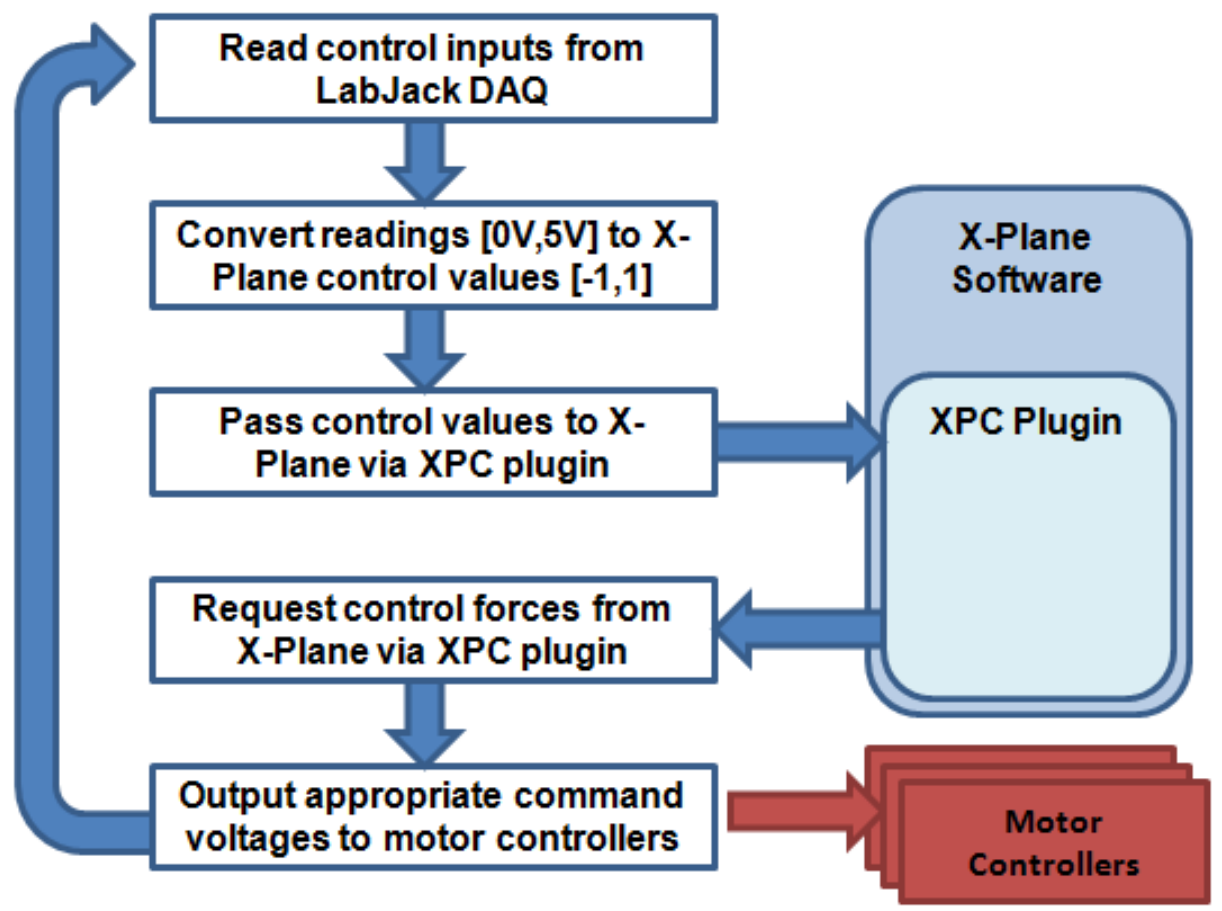

Figure 5.8: Analogue input program flowchart. Note that control loading functionality was also included.

Assuming that the complete range of motion is used, the signal line of each potentiometer should register a voltage in a range from $0 \mathrm{~V}$ (ground) to $5 \mathrm{~V}$ (supply voltage). However, in X-Plane, control positions are represented by a dimensionless number in the range $[-1,+1]$, or occasionally $[0,+1]$ in the case of controls which lack a centre position, such as the throttle. Thus, it was necessary to convert the voltages read from the potentiometers into values acceptable for input into X-Plane. This was accomplished though linear interpolation, as shown in Figure 5.9. (Since the toe brake strain gauge sensors also produced analogue signals in the range $[0 \mathrm{~V}, 5 \mathrm{~V}]$, the toe brake sensors will be treated as potentiometers in the following discussion.) The variables in Figure 5.9 are defined in Table 5.2. 


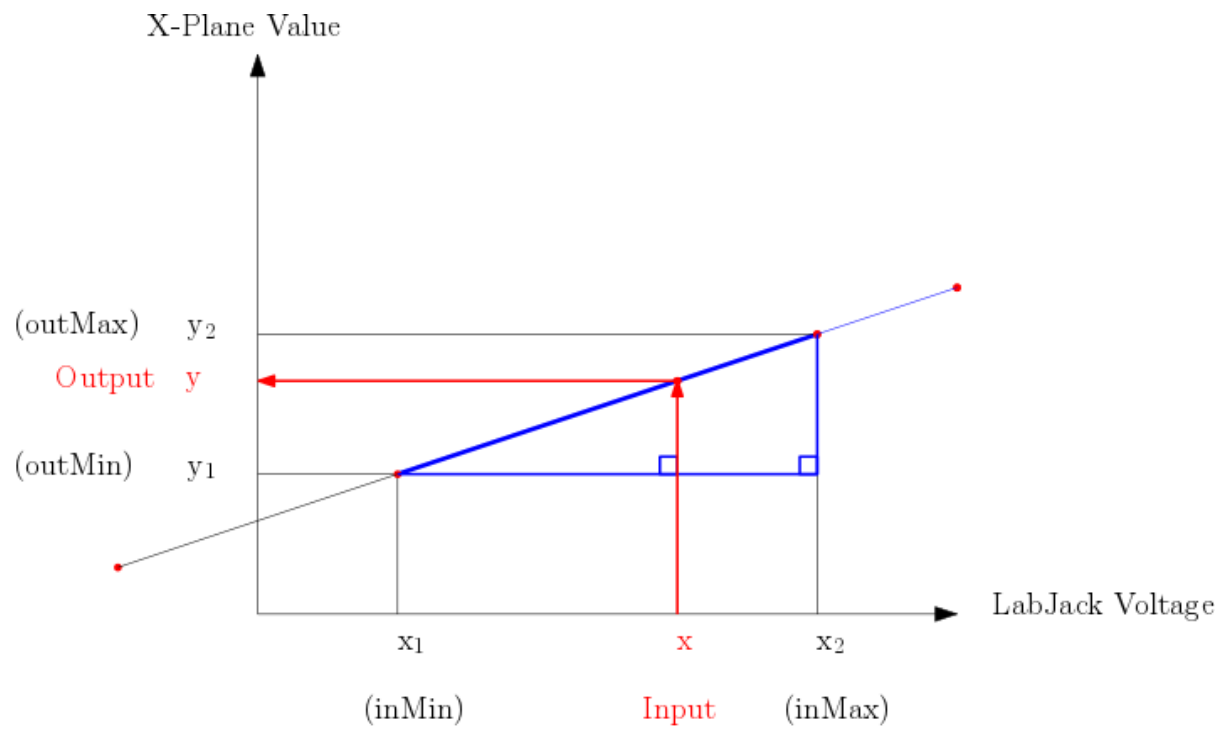

Figure 5.9: A graphical example of linear interpolation. Note that the two right triangles are similar and thus have equal slopes. This allows a known input variable $x$ to be mapped to the corresponding output variable $y$. Image created with Ipe.

Table 5.2: Linear Interpolation Variables

\begin{tabular}{|l|l|l|}
\hline Variable & Name & Description \\
\hline \hline $\mathrm{x}$ & Input & Raw analogue input value from LabJack DAQ \\
\hline $\mathrm{x}_{1}$ & inMin & Minimum expected analogue value \\
\hline $\mathrm{x}_{2}$ & inMax & Maximum expected analogue value \\
\hline $\mathrm{y}_{1}$ & outMin & Maximum allowable output value \\
\hline $\mathrm{y}_{2}$ & outMax & Maximum allowable output value \\
\hline $\mathrm{y}$ & Output & Linearly-interpolated X-Plane value \\
\hline
\end{tabular}

In order to implement this visual algorithm in $\mathrm{C}++$ code, however, an equation was derived. This was done by recognizing that the slopes of similar triangles are equal and rearranging for the output, $y$ :

$$
y=\left(x-x_{1}\right)\left(\frac{y_{2}-y_{1}}{x_{2}-x_{1}}\right)+y_{1}
$$


Or, in plain English:

$$
\text { Output }=(\text { Input }- \text { inMin })\left(\frac{\text { outMax }- \text { outMin }}{\text { inMax }- \text { inMin }}\right)+(\text { outMin })
$$

It should be noted that the above algorithm is identical to that implemented in the Arduino map() function [64].

Once the equivalent X-Plane value was obtained for each analogue input value, XPC functions (such as sendCTRL(), or sendDREFs ()) were then used to pass these values to the XPC plugin, and thence to X-Plane itself. Since the analogue input interface program was run on the same PC as X-Plane, the XPC functions were set up to send the control values to the XPC plugin at the localhost IP address (127.0.0.1).

\subsubsection{Analogue Input Calibration Program}

In an ideal world, each analogue sensor (potentiometer or strain gauge) would use the entirety of its range of motion, resulting in analogue signals ranging exactly between $0 \mathrm{~V}$ and $5 \mathrm{~V}$. However, in the VectorSim, this was found not to be the case. While the analogue signals from most of the sensors ranged from almost $0 \mathrm{~V}$ to almost $5 \mathrm{~V}$, the exact end-of-range values for each sensor were unique. In addition, some sensors - such as the trim wheel potentiometer and the toe brake strain gauges - topped out at approximately $3.5 \mathrm{~V}$.

To deal with these inconveniences, the analogue input interface program initially used end-of-range values which were hard-coded. A feature which is hard-coded is an item which is a permanent part of a program, and cannot be changed without recompilation. However, it was subsequently discovered that the measured end-ofrange values for the potentiometers could vary or "drift" over time, as shown in Figure 5.10. 


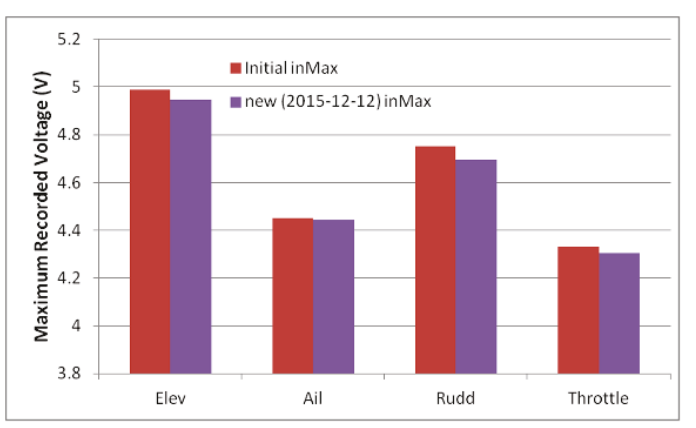

(a) Maximum recorded voltages.

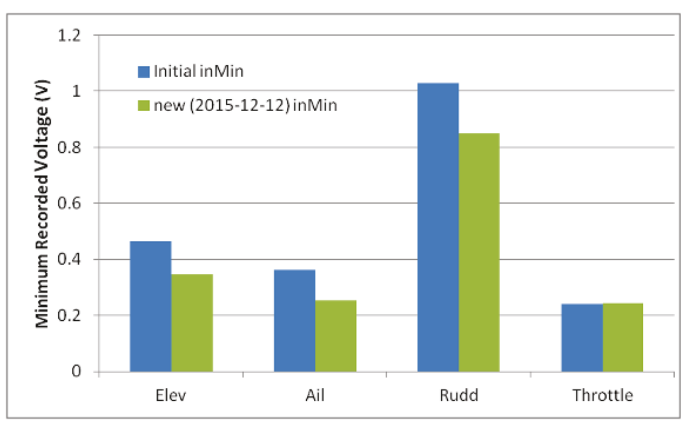

(b) Minimum recorded voltages.

Figure 5.10: Maximum and minimum potentiometer voltages compared over a period of time.

In addition to the issue of drift, hard-coding the end-of-range voltage values into the interface program did not take into account the fact that potentiometers wear out over time and may need replacement. It is not uncommon for simulator potentiometers to be replaced at least once over the life of a simulator. Thus, a straightforward and reliable means of recalibrating the analogue input sensors was necessary.

Before starting to write the analogue input calibration program, the overall calibration task was first broken down into a series of smaller subtasks, itemized below:

1. Connect to the LabJack T7 via its Ethernet interface.

2. Read the values of each of the analogue inputs in turn as the associated sensor is moved through its entire range of motion.

3. Record the minimum and maximum values observed.

4. Write the observed $\min / \max$ values to a calibration text file for later use by the analogue input interface program.

5. Disconnect from the LabJack T7. (This was necessary for the DAQ to be accessed later by the analogue input interface program without restarting the host PC.)

The analogue input calibration program was written in $\mathrm{C}++$. Formatting the 
calibration text file using the extensible markup language (XML) was seriously considered; however, this would have required the use of third-party XML libraries, and increased development time for little benefit. Thus, for simplicity, it was ultimately decided to write the calibration values (max's and min's) to a comma-separated values (CSV) text file instead. The CSV file was structured with variable names and comments such that it was easily human-readable if required for troubleshooting purposes. However, to avoid errors, it is intended that the calibration program will always be used to write to the calibration file. Program flow within the analogue input calibration program is shown in Figure 5.11.

In order to calibrate the analogue inputs on CURVS, the calibration program is first started on the host PC. In order to enable the user to verify that data is being collected, the current, maximum, and minimum analogue voltage values seen so far are continuously output to a console window. The user is prompted to move all analogue controls through their complete range of motion. If satisfied with the $\min / \max$ values collected for each sensor, the user can then opt to save the values displayed on screen to the calibration text file (calib.csv).

Initially, the Windows system("CLS") function call was used to clear the console window before printing the updated voltage values on the next iteration of the analogue input calibration program. Use of this function can be a security risk, however, due to the fact that malicious programs can easily masquerade as the Windows "CLS" command in order to trick the user into inadvertently executing them [65]. While it is intended that the completed CURVS simulation PC network will be offline and isolated from unauthorized access, it was thought best practice to avoid use of system("CLS") entirely. Rapidly writing 15 lines of text to the console on each iteration caused an unacceptable amount of flicker on-screen, however, so the analogue input calibration program was modified to only update the console once every 50th 


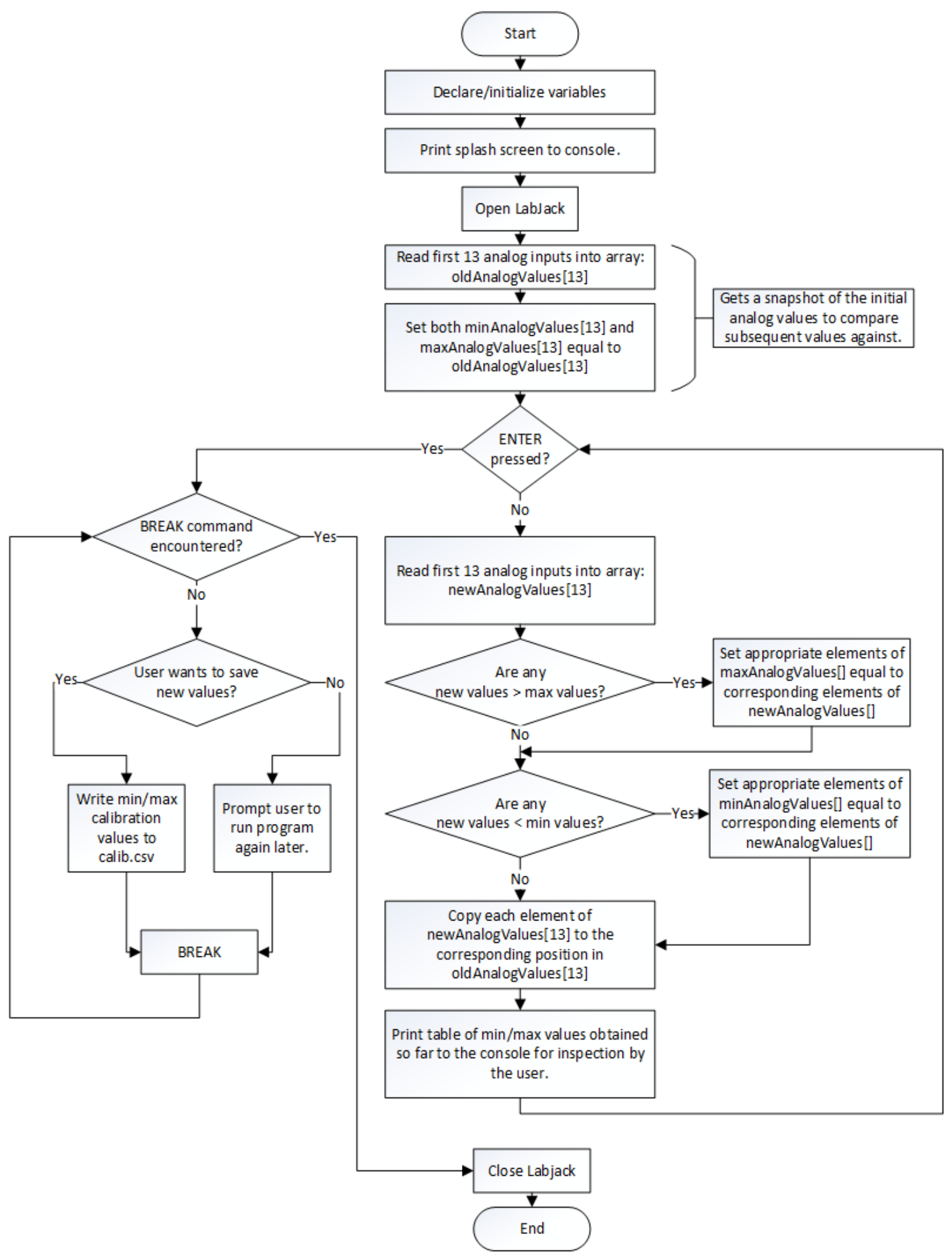

Figure 5.11: Flowchart for the CURVS analogue input calibration program. 
iteration. This resulted in relatively-stable text output to the console while still allowing the program to capture the changing analogue voltages from each sensor at a high enough rate to ensure proper capture if the controls were to be moved quickly.

In the original VectorSim, analogue input calibration was a labour-intensive manual process which required two people. One person would move the cockpit controls through their complete ranges, while the other would read the corresponding analogue voltage values from the screen and manually enter them into a calibration text file. With the new analogue input calibration program, the same task may now be accomplished automatically by a single person in a matter of minutes.

\subsection{Image Generation Computers}

In order to determine the computer hardware most appropriate for the redevelopment of the VectorSim, a list of requirements was first defined. In defining these requirements, the primary considerations were a desire for high framerate, large horizontal field of view, and high visual fidelity. As WWFC indicated that anticipated active usage was expected to be approximately 1000 hours/year, long-term reliability (defined as 10 years) was also given a high priority.

Thus, it was decided that the selected computer system must:

- Run X-Plane 10 on maximum graphical settings;

- Run at high frame rates (minimum 60 FPS desired);

- Render a minimum of three simultaneous cockpit views (forward, left, and right);

- Run image-warping software to correct for a cylindrical projection screen;

- Drive three full-HD projectors;

- Run add-on scenery for maximum realism (e.g., HD Mesh Scenery v.3 or SkyMaxx Pro v.2); and 
- Consist of robust, high-quality components.

In order to keep cost low it was desirable to build a single PC capable of all of the above functions. However, X-Plane 10 is not designed to natively support multiple distinct camera views from a single instance of the software. While it is possible to split a given X-Plane view across several monitors, the user is still restricted to a single virtual view frustum, as illustrated in Figure 5.12. In computer graphics, a frustum is the $3 \mathrm{D}$ region seen and rendered by a given camera view.

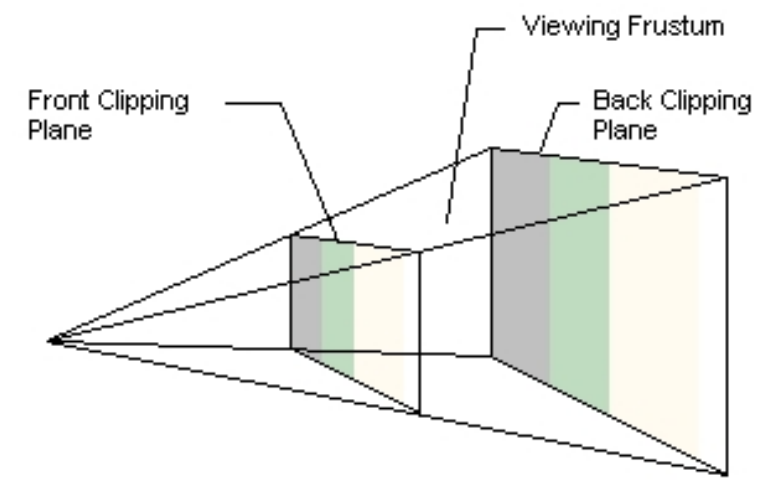

Figure 5.12: A computer graphics view frustum consists of the $3 \mathrm{D}$ volume contained between the clipping planes. Objects within this volume are visible to the virtual camera and are rendered on-screen. In X-Plane, only a single view frustum may be defined. Image from [66].

X-Plane can render a single view frustum with a horizontal field of view up to $170^{\circ}$. However, in order to avoid visual distortions when creating a wraparound cylindrical display system, multiple distinct view frustums must be used. This concept is illustrated in Figure 5.13. While the total width of the display area is $3 x$ for both display systems, the viewing angle at the edge of the field of view is not.

Referring to Figure 5.13a, an image $3 x$ units wide is rendered onto a flat display space consisting of three monitors placed side-by-side. The total field of view available in this system is represented by angle $c_{1}$. Due to the width of the display, the portion of the image at the edge of the field of view is seen at a relatively small angle $b_{1}$ with 


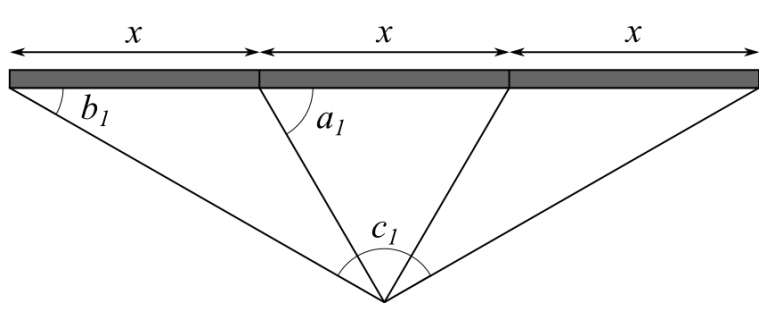

(a)

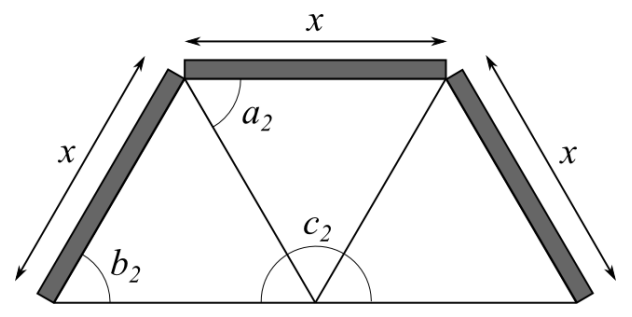

(b)

Figure 5.13: A single-frustum display (a) compared to a triple-frustum display (b). Images created with Inkscape.

respect to the observer. Since the centre display is closer to the observer, the angle $a_{1}$ between the portion of the image at the edge of the centre display and the observer is correspondingly greater.

In Figure 5.13b, the same three displays have been repositioned to wrap around the viewer for a presumably more immersive experience. While the total physical field of view is now greater $\left(c_{2}>c_{1}\right)$, the computer-generated view is still rendered as before (e.g., assuming a flat display of width $3 x$ and field of view $c_{1}$ ). However, the portion of the image at the edge of the outermost display is now being viewed at an angle $b_{2}>>b_{1}$. This inequality between the angle at which the image was rendered to be viewed $\left(b_{1}\right)$ versus the angle at which it is now being viewed $\left(b_{2}\right)$ results in the image appearing distorted or "stretched" towards the edges of the field of view. The viewing angle for the centre monitor remains the same as before $\left(a_{1}=a_{2}\right)$, so the image on the centre monitor remains undistorted.

Thus, in order to create a truly immersive, wraparound display which is free of distortion, multiple distinct view frustums are required. The most common way of achieving this in X-Plane is by using a network of computers, each running a separate copy of the software. One PC is designated as the master, with each of the other PCs on the network acting as slaves. This approach adds expense, however, due to the number of high-performance PCs required. 
The preceding discussion has assumed the use of flat computer monitors or displays. However, for projection onto a curved screen, the image generated by X-Plane must be pre-warped to compensate for the non-flat geometry of the screen. In addition, the overlap between projected images must be blended together by fading out the edges. X-Plane requires a special licence key (1500 USD) to enable its native image warping and edge-blending functionality. However, equivalent functionality may be obtained through use of a third-party program, Immersive Display Pro from Fly Elise-ng (150 EUR). In addition, the MultiView plugin released by Fly Elise-ng allows the generation of multiple distinct view frustums from a single instance of X-Plane [67].

The free version of the MultiView plugin may be used for the creation of a maximum of two distinct views. A licence of Immersive Display Pro is required to unlock the ability to create up to 6 separate views [68]. However, preliminary testing involving two distinct views being run on an older PC equipped with an Intel i5 processor and an NVIDIA 9800GT graphics card with 1 GB of video RAM showed a drop in framerate of approximately $50 \%$ with the plugin enabled. A screenshot from this test is shown in Figure 5.14. Furthermore, for performance reasons, the developer did not recommend using the multiview plugin for more than two projector views, especially at HD resolutions [69]. Thus, in order to ensure a high frame rate of at least 60 FPS using multiple distinct views, it was decided to abandon the idea of a single PC in favour of a traditional networked simulation using multiple copies of X-Plane. 


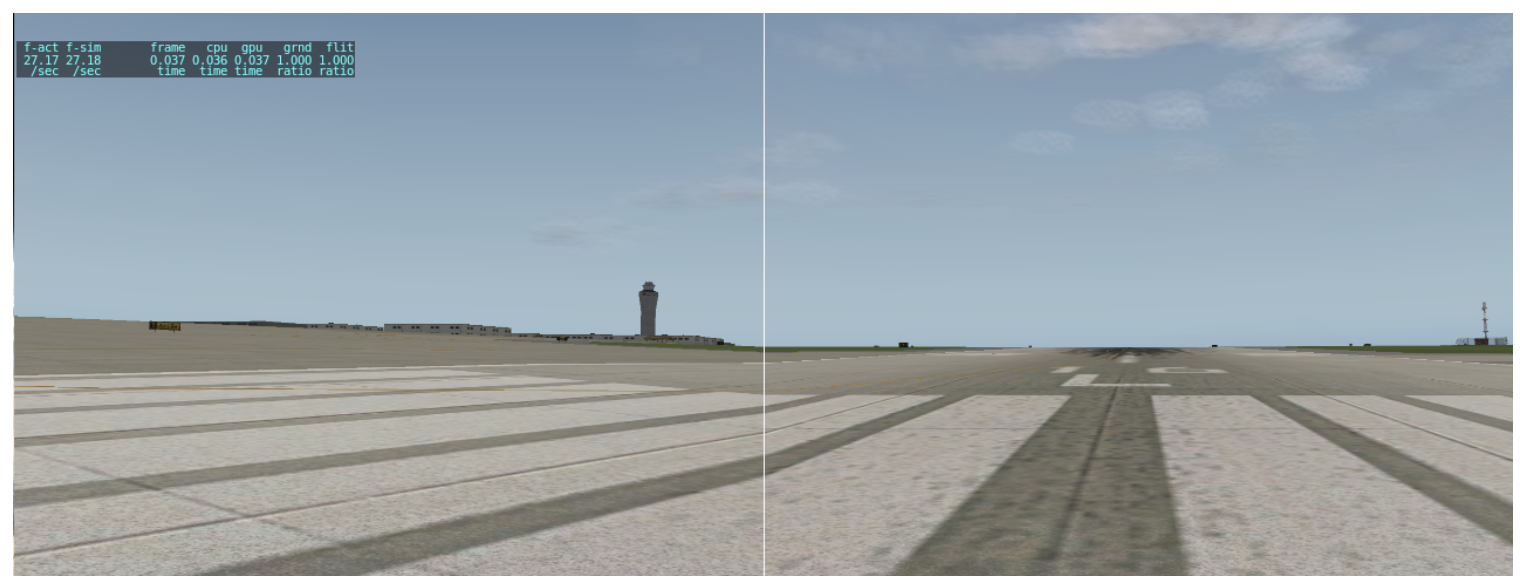

Figure 5.14: Test of MultiView plugin for X-Plane using two $640 \times 480$ pixel images on a single monitor. The view frustum on the left was rotated $45^{\circ}$ to the left, while the frustum on the right is oriented straight ahead. Both frustums have a horizontal field of view of $45^{\circ}$.

\subsubsection{Recommended System Requirements}

At the time that the specifications for the CURVS PCs were being developed, the recommended system requirements for X-Plane 10 were as given in Table 5.3. It is interesting to note that as of this writing, the recommendations have been updated to call for 16-20 GB of RAM and at least 4 GB of video RAM (VRAM).

Table 5.3: Recommended system requirements for X-Plane 10 as of February 2015.

\begin{tabular}{|l|l|}
\hline & Recommended Specifications \\
\hline CPU & $3+\mathrm{GHz}$, multi-core (or multi-CPU) \\
\hline RAM & $8-16 \mathrm{~GB}$ \\
\hline Video & $2-4 \mathrm{~GB}$ VRAM, DirectX 11 \\
\hline
\end{tabular}

In addition to X-Plane, it was also decided to use HD Mesh Scenery version 3, a high-resolution add-on scenery package for X-Plane [70]. Permission to use this product for research and ultimate commercial use at WWFC was graciously granted by its developer, Andras Fabian. In order to use HD Mesh Scenery in conjunction with other scenery packages, at least 16 GB of RAM was recommended. 


\subsubsection{Part Selection}

X-Plane performance depends most strongly on the speed at which a single program thread can be executed [71]. This is due to the fact that X-Plane runs mainly on a single core, with multiple cores being used only while loading a simulation. This behaviour may be observed by monitoring CPU usage while X-Plane is running. Thus, it was decided that central processing unit (CPU) clock speed would take precedence over number of cores in the CPU selection process. Based on a perusal of processor reviews and recommendations in the computer gaming literature, the Intel i5 4690K quad-core CPU was selected. This processor is a top-tier unit suitable for gaming [72]. Originally, a higher-performance Intel i7 4790K quad-core CPU had been selected; however, after the decision was made to use a network of multiple computers, the CPU-requirements for the individual PCs were lowered given the reduced processing load per PC. The "K" designation indicates an unlocked processor suitable for overclocking. Overclocking is a performance-enhancing technique whereby computer processors are run at speeds beyond their rated limits. In order to promote long-term system longevity, none of the CURVS CPUs were overclocked. Rather, unlocked CPUs were chosen due to their cost-effectiveness at the time of purchase, and also due to the fact that unlocked CPUs are typically of higher quality than their locked brethren [73].

The Freezer i11 aftermarket CPU cooler from Arctic Cooling was selected due to its increased cooling performance compared to a typical stock Intel cooler, large radiator size, ability to be mounted in-line with case airflow, and cost-effectiveness [74]. In addition, at $8.5 \mathrm{~W} /(\mathrm{m} \cdot \mathrm{K})$, the included MX-4 thermal interface compound featured the highest heat conductivity rating of all compounds evaluated, and was rated to last at least 8 years [75]. 
The ASUS Sabertooth Z97 Mark 2 motherboard was selected for its overall robustness for long-term use [76]. It featured use of military-grade components, electrostaticdischarge (SSD) protection circuitry, and a 5-year warranty.

The NVIDIA GeForce GTX 960 video card with 4 GB of VRAM by EVGA was selected due to its high clock speed (base: $1279 \mathrm{MHz} /$ boost: $1342 \mathrm{MHz}$ ) and favourable price-to-performance ratio [77]. This card also included a metal backplate, which was desirable to prevent warping of the card in long-term use.

A total of 16 GB of Kingston HyperX Fury DDR3 RAM was selected for each PC due to its price-to-performance ratio in conjunction with the positive reputation of its manufacturer.

In order to decrease the long load times typical of X-Plane, it was decided that a solid-state hard drive (SSD) would be used in each of CURVS PCs. An SSD consists entirely of solid-state flash memory and contains no moving parts. As such, it is significantly faster than a traditional, mechanically-based hard disk drive (HDD). The Samsung 850 Evo 500 GB SSD was selected to allow for a full installation of both the X-plane default world scenery and the add-on HD Mesh Scenery. This SSD also included a 5-year warranty.

The Seasonic G-750 Gold power supply unit (PSU) was selected due to its high build quality, high electrical efficiency (80 PLUS ${ }^{\circledR}$ Gold rating), and 5-year warranty [78].

A complete listing of the components selected for use in the CURVS simulation PCs are shown in Table 5.4. Four identical PCs were built to these specifications by a local computer store for a total cost of approximately 1760 CAD per unit. 
Table 5.4: CURVS PC component list.

\begin{tabular}{|l|l|}
\hline Item & Component \\
\hline CPU & Intel Core i5 4690K (Unlocked, Quad Core, 3.5 GHz / 3.9 GHz) \\
\hline CPU Cooler & Arctic Cooling Freezer i11 \\
\hline Motherboard & ASUS Sabertooth Z97, Mark 2 \\
\hline Video Card & EVGA GeForce GTX 960 SuperSC ACX 2.0+ (4 GB GDDR5 VRAM) \\
\hline RAM & Kingston HyperX Fury (2x8 GB DDR3-1866, CL10, Dual Channel) \\
\hline SSD & Samsung 850 Evo Series MZ-75E500B/AM (500 GB, SATA III) \\
\hline Power Supply & Seasonic G-750 Gold (750W, ATX, 80PLUS, Modular) \\
\hline DVD Drive & ASUS DRW-24F1ST 24X SATA DVD Writer \\
\hline Case & Norco RPC-450 (4U Rackmount Server Case) \\
\hline
\end{tabular}

\subsubsection{Simultaneous Power-on}

The cases selected for the flight simulation PCs were equipped with locking metal grilles, with the power buttons hidden behind the grilles. However, for the convenience of the user, it was desirable that all of the simulation PCs be able to power-on using a single, unobstructed power button.

Modern ATX-style power supplies are designed to work in conjunction with a momentary-contact pushbutton [79]. Rather than conducting power to the power supply directly, this "soft switch" merely connects the "PS_ON" line on the power supply to ground. When this occurs, the power supply turns itself on. A commerciallyavailable, low-cost remote PC power button was connected to the master flight simulation PC. The button was affixed next to the keyboard at the instructor station. Rather than use a separate power button for each PC, the "wake-on-LAN" functionality of the PC motherboards was utilized. Once started with the remote button, the master PC would then wake all other PCs on the simulator's local area network (LAN). 


\subsubsection{Performance Metrics}

In order to obtain a quantitative indication of the graphical power of the CURVS simulation PCs, the "3DMark Fire Strike" (v. 1.1) PC benchmarking application by Futuremark Corporation was run [80]. This test provides a unitless numerical score which may be used for relative comparisons with other systems. Higher scores indicate better performance. For consistency, all unnecessary background processes were stopped, the test run twice, and the individual scores averaged. The latest available graphics driver was also used. The results are shown in Table 5.5.

Table 5.5: CURVS PC benchmarks (3DMark Fire Strike v. 1.1 )

\begin{tabular}{|c|c|l|l|}
\hline Test \# & Score & Result URL & Average Score \\
\hline 1 & 6,696 & http://www.3dmark.com/3dm/12384169 & \multirow{2}{*}{6701.5} \\
\hline 2 & 6,707 & http://www.3dmark.com/3dm/12384276 & \\
\hline
\end{tabular}

The primary purpose of this benchmark was to provide a point of comparison with future PCs which may be built by the ADL or are currently operated by other researchers.

The effectiveness of the system cooling fans and aftermarket heatsinks was assessed using the Perfmonitor2 utility program from CPUID [81]. The aftermarket CPU cooler in conjunction the pre-installed case fans were found to keep CPU temperatures for all four PCs between $40^{\circ} \mathrm{C}-50^{\circ} \mathrm{C}$, well below the maximum case temperature of $72.72^{\circ} \mathrm{C}$ specified for the $\mathrm{CPU}[82]$.

Approximate X-Plane RAM usage by the master PC (using HD Mesh Scenery and custom scenery for Waterloo Wellington airport) is summarized in Table 5.6. For test consistency, a standard X-Plane scenario was set up as follows:

- airport: CYKF

- runway: 08 
Table 5.6: Approximate X-Plane RAM usage.

\begin{tabular}{|l|l|l|}
\hline & RAM Usage (GB) & VRAM Usage (GB) \\
\hline Default Cessna 172 & 1.8 & 2.6 \\
\hline Airfoil Labs Cessna 172(low-res version) & 2.1 & 3.0 \\
\hline
\end{tabular}

- preset: "few clouds"

- visibility: 20 statute miles

The X-Plane rendering settings used on the CURVES visual PCs are shown in Figure 5.15. Using these settings, a consistent framerate of approximately 60 FPS was achieved on the image generation PCs. Thus the framerate design requirement was satisfied.

It is important to note that when using multiple rendering PCs in a simulation network, the herds of deer which X-Plane can generate are not synchronized between rendering PCs. This results in herds of deer which run off the edge of one projector and vanish into thin air. Thus, this feature should be disabled in multi-PC simulation setups. 


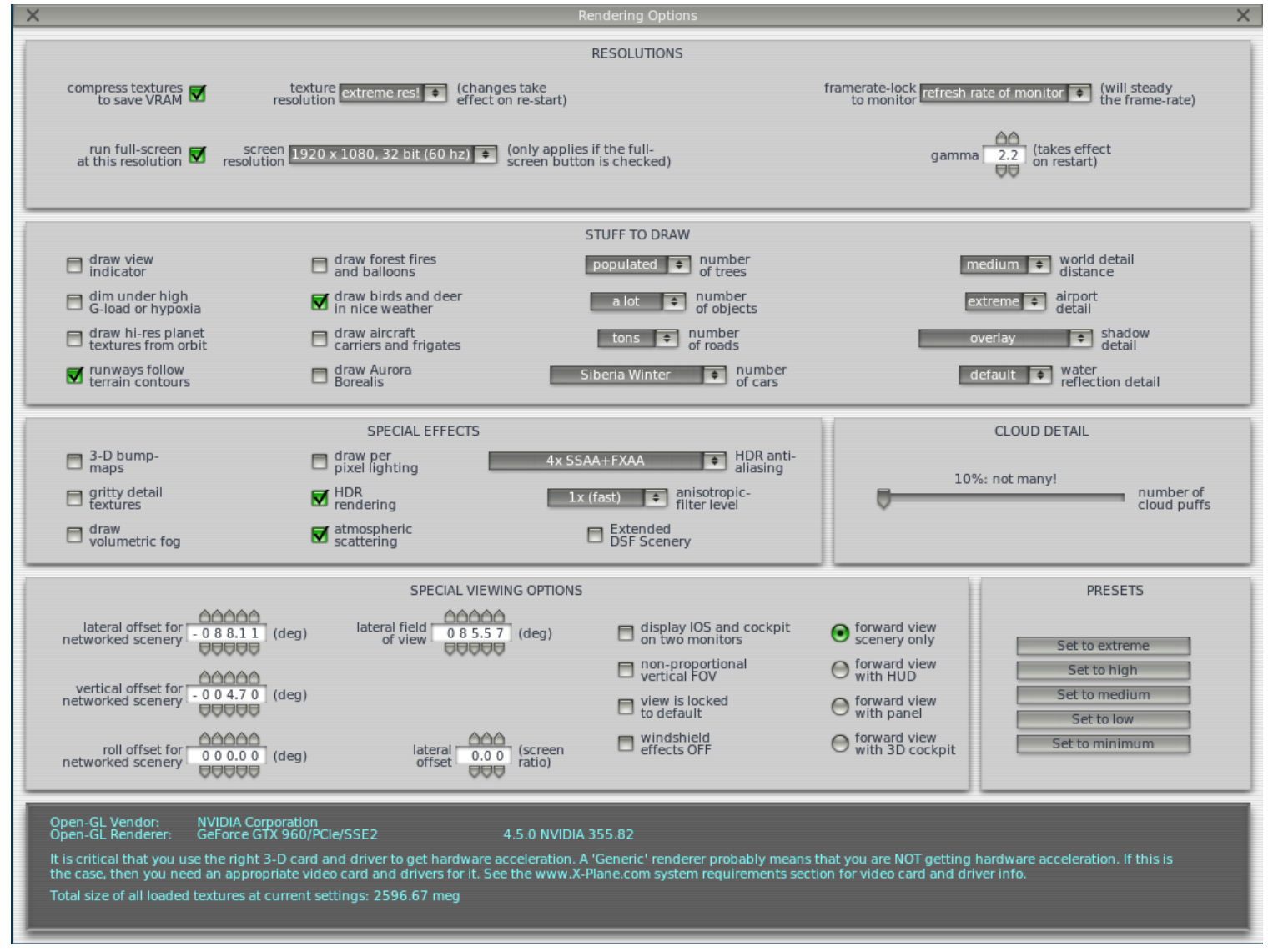

Figure 5.15: X-Plane rendering settings for the left-hand visual PC. Settings for other PCs are similar except for the scenery view offsets. Note that rendering of birds and deer should be disabled. 


\subsection{Control Loading}

In a Cessna 172 aircraft, the yoke and rudder pedals are directly connected by means of cables to the individual control surfaces. Rotation of the yoke causes aileron deflection and controls roll motion, while fore-and-aft motion of the yoke causes elevator deflection and controls pitch motion. The rudder pedals are connected to the rudder on the vertical stabilizer, and control yaw motion. Since the controls are mechanically connected to their associated control surfaces, the cockpit controls can be back-driven by moving the control surfaces. Such a control system is known as a reversible control system. The force required for the pilot to move the controls therefore varies depending on both the speed at which the aircraft is moving through the air (airspeed), and the amount by which the controls are moved. In a simulator, the control loading system - if present - is responsible for applying appropriate forces to the yoke and rudder pedals to mimic this effect. Many lower-cost flight training devices use springs to approximate the control forces which should be felt; however, this approach lacks fidelity since spring stiffness is constant. This makes the controls feel equally stiff at all times, even when the simulated aircraft is stationary on the ground and the controls should therefore require little force to move.

The aerodynamic force on an object moving through the atmosphere is directly proportional to the air density times the velocity squared [83]. Since the pilot's flight controls in a Cessna 172 are directly connected to the control surfaces via mechanical linkages, the force which must be exerted by the pilot to deflect a control surface by a given amount in flight also varies with the square of the airspeed [84]. For simplicity, the forces exerted by the pilot on either yoke or rudder will be referred to in general as "stick forces," as use of a control stick in aviation predates the yoke. Of course, the stick forces will differ from the forces on the control surfaces depending on the mechanical advantage provided by the linkages. 
By way of example, elevator stick force is given by the following equation:

$$
F_{\text {sticke }_{e}}=G \cdot C_{H_{e}} \cdot \eta_{h_{t}} \cdot \frac{1}{2} \cdot \rho \cdot V^{2} \cdot A_{e}
$$

where $\mathrm{G}$ is a gearing and conversion factor which accounts for the mechanical advantage of the control linkages and converts moment to force, $C_{H_{e}}$ is the non-dimensional hinge moment coefficient, $\eta_{h_{t}}$ is the horizontal tail efficiency factor to correct for the disturbed airflow over the tail, $\rho$ is air density, $V$ is the airspeed, and $A_{e}$ is the elevator area aft of the hinge line $[85,86]$.

Assuming a linear relationship, the hinge moment coefficient may be represented by the following equation:

$$
\begin{aligned}
C_{H_{e}} & =C_{H_{e_{0}}}+\left(\frac{\partial C_{H_{e}}}{\partial \alpha_{h_{t}}}\right) \alpha_{h t}+\left(\frac{\partial C_{H_{e}}}{\partial \delta_{e}}\right) \delta_{e}+\left(\frac{\partial C_{H_{e}}}{\partial \delta_{t}}\right) \delta_{t} \\
& =b_{0}+b_{1} \alpha_{h_{t}}+b_{2} \delta_{e}+b_{3} \delta_{t}
\end{aligned}
$$

where $b_{0}$ is the elevator hinge moment coefficient at zero angle of attack and zero deflection, $\alpha_{h t}$ is the angle of attack of the horizontal tail, $\delta_{e}$ is the deflection of the elevator, and $\delta_{t}$ is the deflection of the elevator trim tab. For simplicity, the three partial derivatives in Equation 5.4 are represented by $b_{1}, b_{2}$, and $b_{3}$ respectively in Equation 5.5. These partial derivatives represent the changes in the hinge moment coefficient with respect to angle of attack, elevator deflection, and trim tab deflection, respectively. Note that for a symmetrical airfoil such as that used on the horizontal tail of a Cessna $172, b_{0}$ is zero [87].

Returning to stick force Equation 5.3, the constant terms may be grouped together 
and the hinge moment expanded as follows:

$$
F_{\text {sticke }_{e}}=\left(\frac{1}{2} \cdot G \cdot \eta_{h_{t}} \cdot A_{e}\right) \cdot \rho \cdot V^{2} \cdot\left(b_{1} \alpha_{h_{t}}+b_{2} \delta_{e}+b_{3} \delta_{t}\right)
$$

As a first approximation, the dependence of hinge moment on both angle of attack and trim tab deflection was ignored. This allowed the corresponding terms to be removed from Equation 5.6:

$$
F_{\text {sticke }}=\left(\frac{1}{2} \cdot G \cdot \eta_{h_{t}} \cdot A_{e}\right) \cdot \rho \cdot V^{2} \cdot\left(b_{\gamma} \alpha_{h_{t}}{ }^{0}+b_{2} \delta_{e}+b_{3} \delta_{t}{ }^{0}\right)
$$

Since the dependence of hinge moment on angle of attack was ignored, the effects of aerodynamic stall on stick force were not modelled. However, the CURVS FTD is intended primarily for ab-initio flight training. In this type of training, stalls and spins are only performed at low speeds, when control forces are already low due to the $V^{2}$ term without accounting for the stall explicitly. In any case, notice that in general stalls and spins are often performed in an actual aircraft rather than a simulator due to the fact that a simulator without motion cannot reproduce the real-life accelerations felt by the student during these manoeuvres. So-called "accelerated," or high-speed stalls are not performed in ab-initio flight training.

In X-Plane, trim tab deflection is not explicitly modelled; rather, its effect is simulated by simply deflecting the elevator as a whole. Thus, the dependence of hinge moment on trim tab deflection can be removed with no adverse consequences.

Finally, the constant terms of Equation 5.7 were grouped into a single constant, $K$ :

$$
F_{\text {sticke }_{e}}=K \cdot \delta_{e} \cdot \rho \cdot V^{2}
$$


Thus, we have a mathematical relationship between airspeed (available from XPlane) and what we want to find, namely the stick force which must be exerted by the pilot to obtain a given deflection of the elevator. The equations for aileron and rudder force are similar.

In X-Plane, control surface deflection is represented by a non-dimensional floatingpoint number in the range $[-1,+1]$, with the centre (undeflected) position represented by a value of zero. Thus, a deflection of +1 represents maximum deflection, and will result in maximum stick force for a given airspeed and air density. Similarly, a deflection of -1 represents a full control deflection in the opposite direction.

In order to determine the value of the constant $K$, only one flight test data point is required. If stick force felt for a given control deflection at a specific reference airspeed is known, the equation may then be solved for the value of constant $K$. This constant may then be used to calculate the control forces corresponding to any combination of airspeed and control deflection, as illustrated in Figure 5.16. Since it is based on experimental data, this approach eliminates the need to know the geometry of the physical linkages connecting the control stick to the control surfaces. This method of calculating control loading forces appears to have been implemented in X-Plane.

While X-Plane stick force datarefs are available, as shown in Table 5.8, the values of these datarefs are not calculated by default. Before these datarefs can be used, calculation of control forces must be specially enabled for the desired aircraft model using the Plane-Maker program. Plane-Maker is a standalone application included with X-Plane which allows the user to edit existing aircraft models or create new ones. The appropriate settings page in Plane-Maker is shown in Figure 5.17.

In order to calculate control forces, X-Plane requires that the force required to do a full control deflection at a given airspeed be known. (This data pair forms the "known datapoint" in Figure 5.16.) Damping values are also required, as X-Plane will not calculate stick forces unless the damping values are changed from their default 


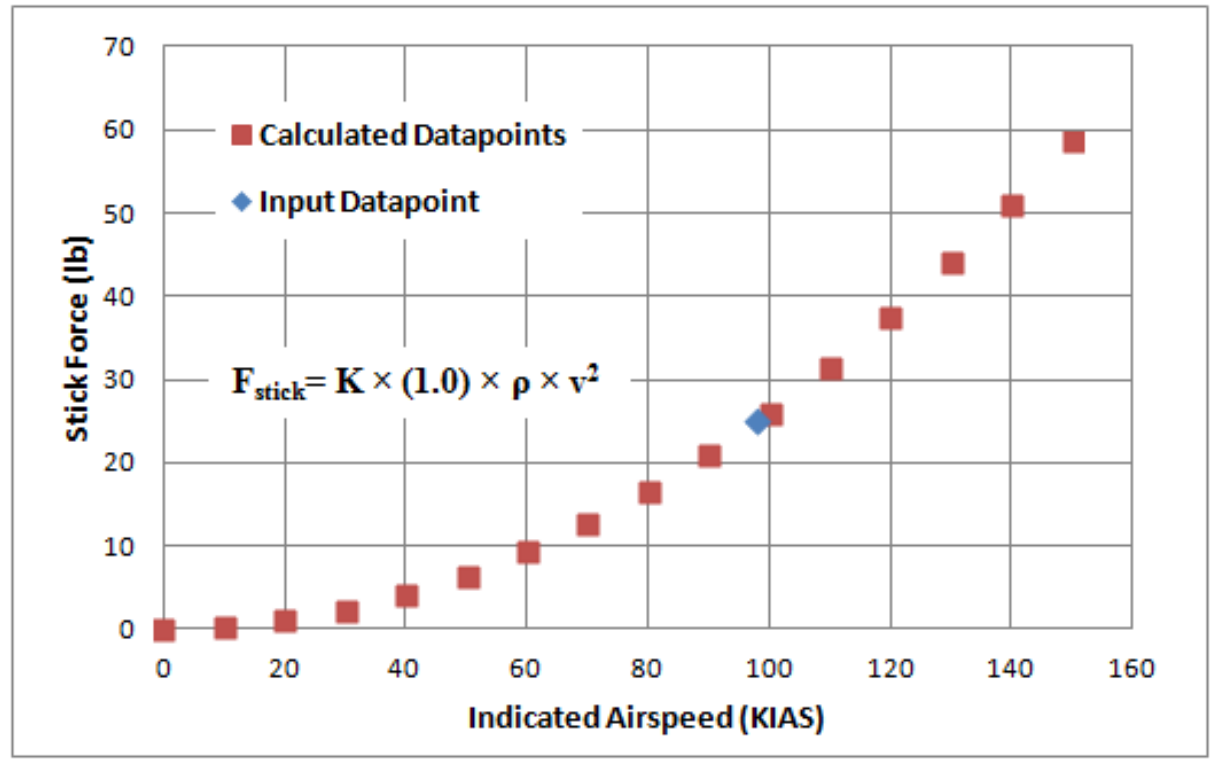

Figure 5.16: Example of calculating stick force based on airspeed, assuming full control deflection $(+1.0)$. The known datapoint (used to calculate $K$ ) is indicated by a blue diamond symbol.

Table 5.8: X-Plane stick force datarefs.

\begin{tabular}{|l|l|l|}
\hline & Dataref & Units \\
\hline Pitch Force & sim/flightmodel/misc/act_frc_ptch_lb & $\mathrm{lb}$ \\
\hline Roll Force & sim/flightmodel/misc/act_frc_roll_lb & $\mathrm{lb}$ \\
\hline Yaw Force & sim/flightmodel/misc/act_frc_hdng_lb & $\mathrm{lb}$ \\
\hline
\end{tabular}




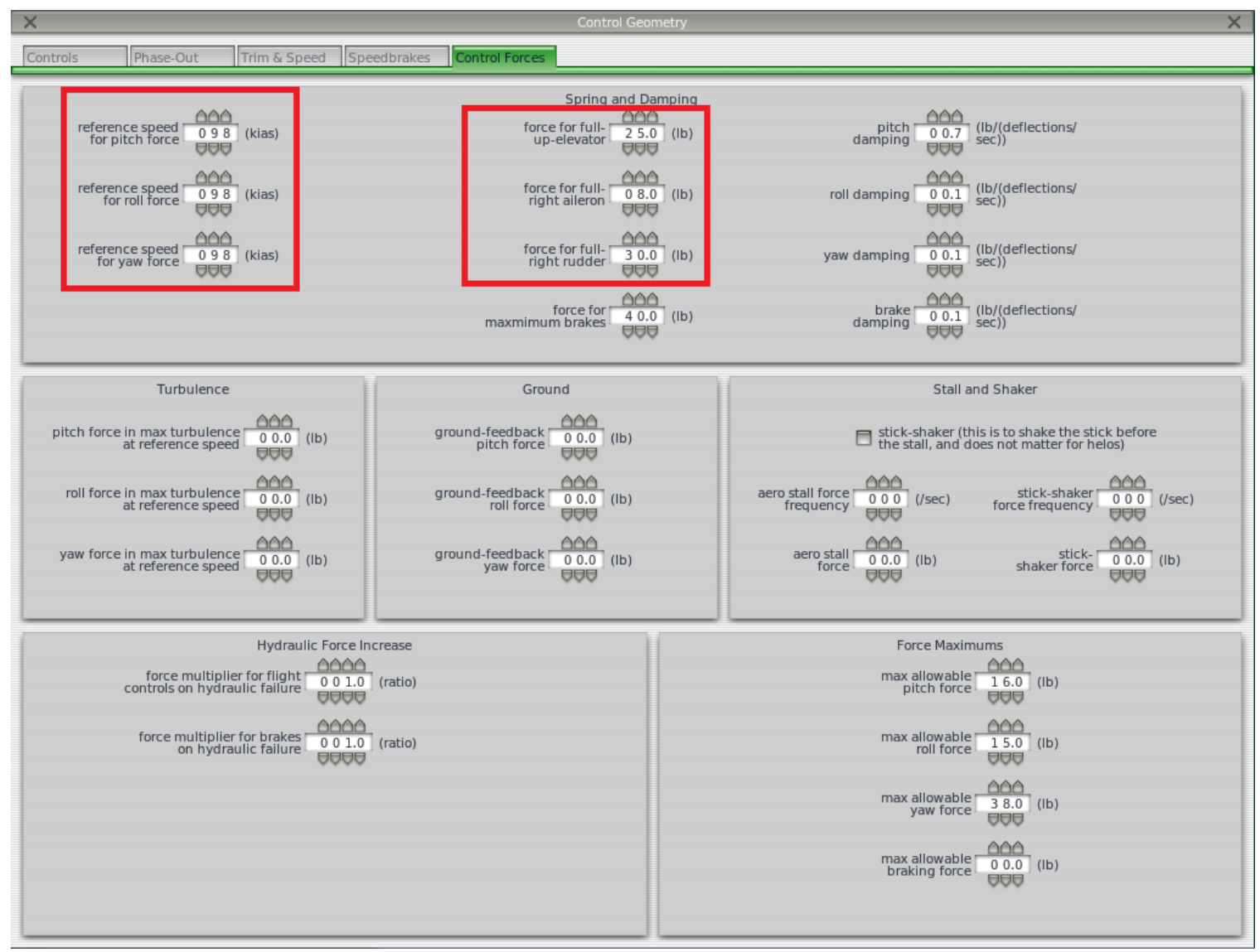

Figure 5.17: X-Plane control force settings in Plane-Maker. 
of zero. These values are difficult to obtain in practice, however, as the making of full control deflections while measuring the force required to do so is not one of the standard flight tests required by Transport Canada for FTD qualification.

Although the specific values required by X-Plane to enable stick force calculations were not readily available, an iterative technique was developed which was able to successfully determine appropriate values for these parameters. This technique was based on original flight test data which had been used during qualification testing of the original VectorSim [7].

For simplicity, the X-Plane reference airspeed for a full control deflection was chosen to be equal to the manoeuvring speed of a Cessna 172. This choice eliminated the need to tune two variables at the same time. The manoeuvring speed $V_{a}$ of an aircraft is defined as the maximum speed at which a full or abrupt control deflection may be made without overstressing the airframe [88]. For a Cessna 172 operating in the utility category with a gross weight of $2,200 \mathrm{lb}$, the manoeuvring speed is $98 \mathrm{kts}$ indicated airspeed (KIAS).

For certification purposes, aircraft are subdivided into four categories, normal, utility, aerobatic, and commuter [89]. A Cessna 172 may be operated in either the normal or utility categories, depending on how the aircraft is configured with respect to weight and balance. The category in which an aircraft is operated determines the manoeuvres which can be safely performed. The utility category of operation allows semi-aerobatic manoeuvres such as spins. As spin recovery techniques are taught as part of the Canadian private pilot licence (PPL), the utility category manoeuvring speed was selected.

In order to determine an appropriate value for the force required for a full upelevator deflection, test 2c(1) ("power change dynamics") from the Federal Aviation Administration (FAA) Advisory Circular (AC) 120-45A was performed [90]. This test is equivalent to test $2 \mathrm{C}(1)$ in the Transport Canada Aeroplane and Rotorcraft 
Simulator Manual (TP9685e) and is designed to verify that the pitch force required in an FTD to maintain altitude when full engine power is applied is comparable to that experienced in the actual aircraft [1]. To execute this test, an X-Plane simulation was set up to match the flight test conditions (altitude, RPM, etc.) specified in the original Reference Data Report for the VectorSim [7]. Full power was then applied using the throttle. Forward pressure was applied on the yoke using a digital scale in order prevent a climb and maintain a constant altitude. The recorded force value was then compared with the force recorded in the flight test data. The X-Plane value for the force required for full up-elevator deflection was then adjusted by a small amount, and the test performed again until the recorded yoke force was approximately the same as that recorded in the flight test data. (Exact force readings were found to be impossible to obtain due to measurement noise when hand-holding the scale.)

Due to time constraints, the X-Plane force values for maximum aileron and rudder deflections were tuned intuitively based on the author's personal flight experience, without using any tests from AC 120-45A. However, the aileron and rudder forces thus obtained were evaluated as realistic by several real-world pilots.

The damping values were tuned via a trial-and-error method starting from 0.1 $\mathrm{lb} /($ deflections/sec), the smallest possible damping value permitted in X-Plane. These values were iteratively increased in an attempt to keep the control loading stable and prevent excessive oscillation of the flight controls without interfering with the pilot's ability to move the controls by hand.

\subsubsection{Reverse-engineering the Control Loading System}

A search was conducted for any documentation regarding the original Thompson OMNIDRIVE servomotors and servomotor drives used in the control loading system. While the servomotors and drives were found to have been discontinued by their original manufacturer, the installation manual was located on the website of Artisan 
Technology Group, a company which specializes in the support and sales of legacy industrial equipment [91].

Upon further investigation, the control loading system was found to be identical to that used in the Piper Warrior flight training devices which had also been built by the former Vector Training Systems. Using original VTS Piper Warrior documentation provided by Jared Wilhelmi in conjunction with the installation manual for the original motor controllers, it was possible to reverse-engineer the existing system and determine how it was originally intended to work. The original control loading system is illustrated schematically in Figure 5.18.

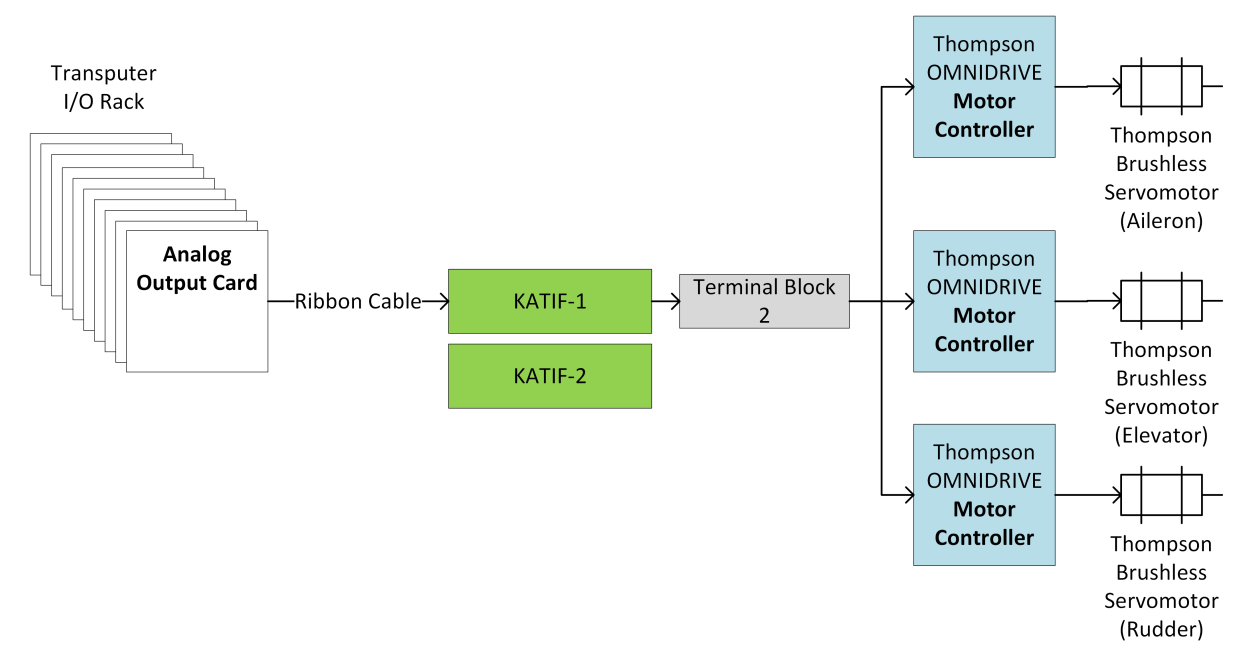

Figure 5.18: Original VectorSim control loading system architecture.

\subsubsection{Testing the Existing Control Loading Motors}

As the original FTD had been in storage for a number of years - some of which had been spent in an unheated building - it was necessary to verify that the control loading motors and drives were still functional. The installation manual revealed that the servomotor drives were controlled via a \pm 10 VDC reference signal. These signal lines were broken out at what the original documentation referred to as "Terminal 
Block 2," shown previously in Figure 2.9. This terminal block facilitated connections between the original transputer-based control system located at the front of the FTD, and the motor drives located at the rear. Using the Piper Warrior FTD documentation as a guide, the transputer control lines were disconnected from the terminal block, and replaced with test circuitry designed to apply DC voltages to the appropriate terminals on the motor drives. A rocker switch was used to activate the ENABLE inputs on the motor drives and allow them to operate. The test circuitry used is illustrated schematically in Figure 5.19. A 9 VDC power supply and two voltage dividers were used to stimulate the command ("CMND") inputs on the aileron and elevator motor drives, while an adjustable 10 VDC power supply was used for the rudder drive. While a more elegant solution could have been devised, due to the temporary nature of the test, it was most expedient to simply use parts and equipment which were readily available.

The test apparatus was set up as shown in Figure 5.20. Note the ENABLE switch taped to the frame of the FTD in Figure 5.20c. This switch was later replaced with an emergency stop ("E-Stop") button. 


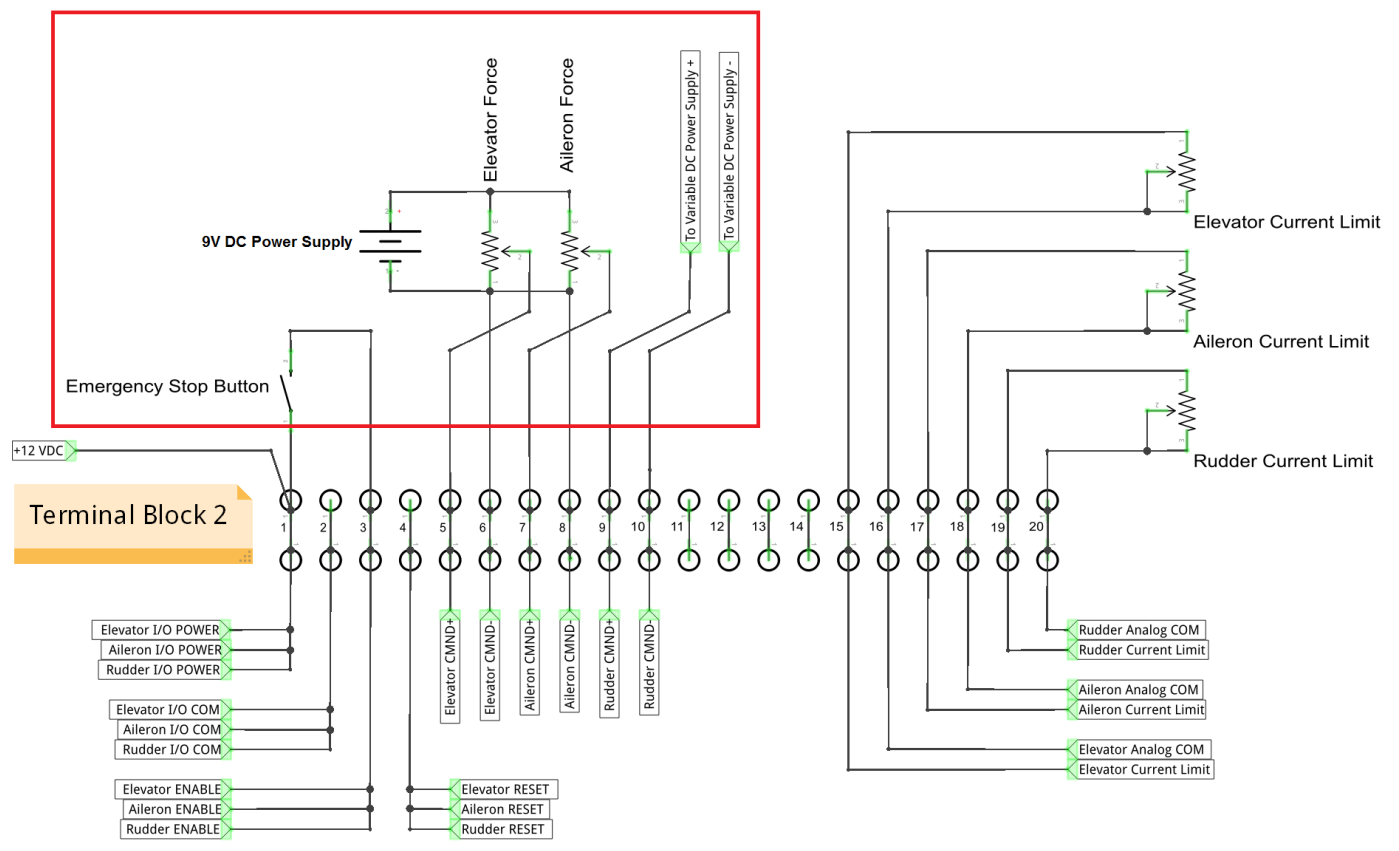

fritzing

Figure 5.19: Circuitry used to test the existing control loading system. Red box indicates components added for the test. Note that connections to terminals 2 , 4, and 11 - 14 on Terminal Block 2 have been omitted for clarity. Image created with Fritzing. 


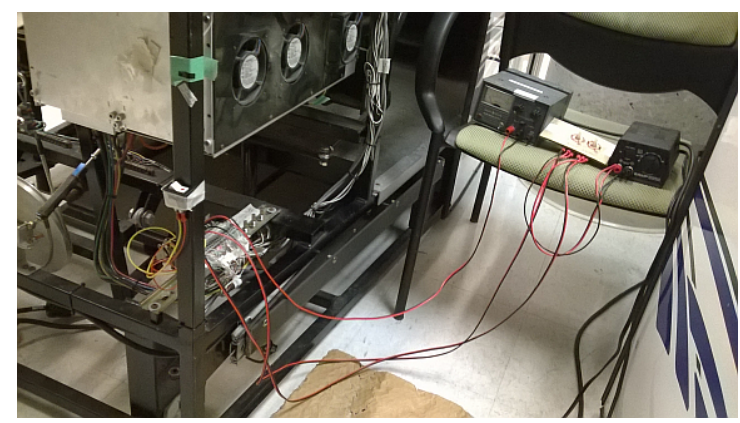

(a) Overall view of test apparatus.

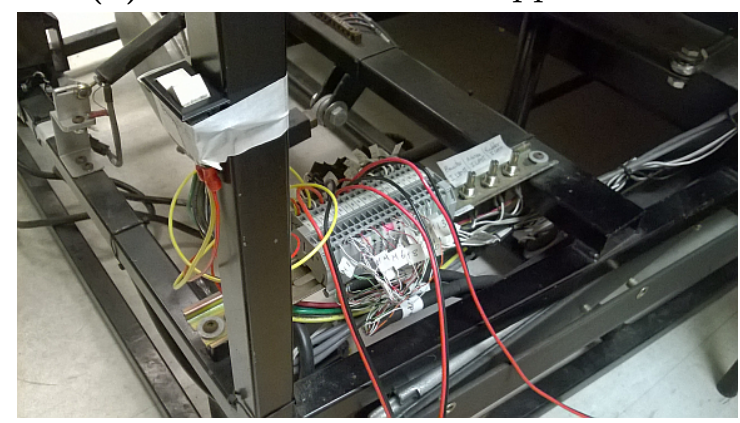

(c) Terminal Block 3 and ENABLE switch.

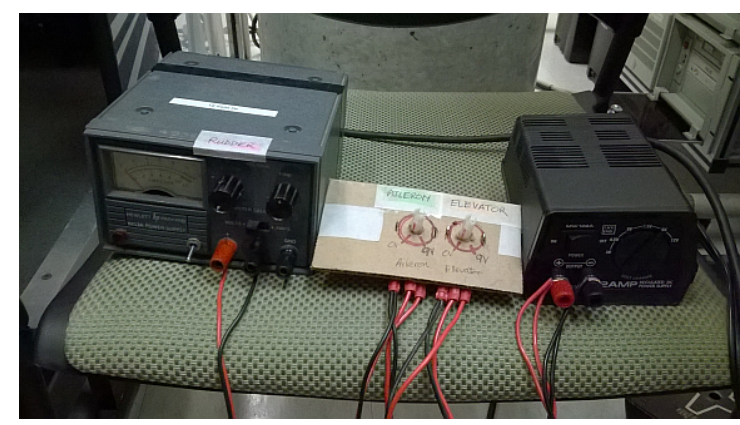

(b) Power supplies and voltage dividers.

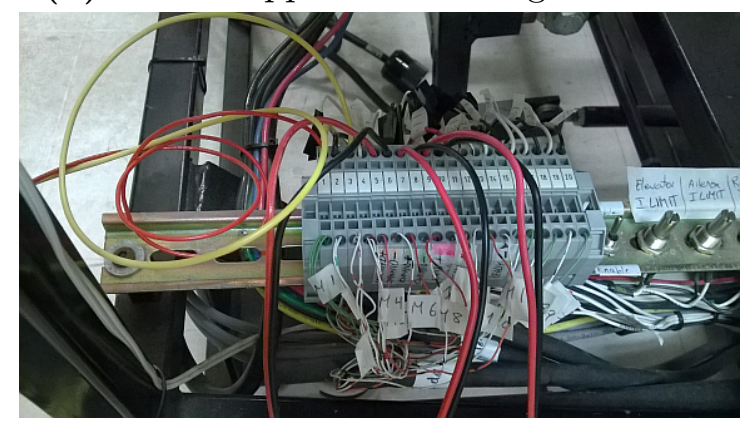

(d) New connections to Terminal Block 3.

Figure 5.20: Setup of the apparatus used to test the control loading servo drives and associated motors.

Using this test apparatus, it was possible to activate the control loading motors and cause them to turn, moving the flight controls. Due to its design, the test apparatus was unable to reverse the current direction, so the motors were rotated in only one direction. Increasing the voltage fed to the CMND inputs on the motor drives caused the forces applied to the flight controls to increase. This indicated that the motor drives were operating in torque mode, as opposed to the velocity or position modes which they were also capable of. This was important to verify, since the legacy Thompson OMNI LINK configuration program required to access drive settings was unavailable. (This program intended for the Windows 3.1 operating system was finally obtained from the manufacturer near the end of the project.)

Thus, despite its long period of disuse, the control loading system was verified to still be fully functional, and capable of being reused in the redeveloped FTD. 


\subsubsection{Quantifying Control Loading Motor Output Torques}

The torque output by the control loading servomotors was proportional to the DC voltage applied across the CMND+ and CMND- inputs on the motor driver units. However, no engineering drawings of the linkage system between the motors and the flight controls were available. Thus, even if the relationship between command voltage input and motor torque output had been known, it would not have been possible to determine the torque available at the pilot's controls without disassembling the linkages and measuring the dimensions of each link. Thus, it was decided to quantize this relationship experimentally. This was done using a scale connected between the selected flight control and a rigid anchor point.

For each control axis, a digital scale was installed between the appropriate control and a suitable anchor point in the cockpit. The yoke was found to be already equipped with a hole in the right handle for attaching a scale. Holding the other side of the scale by hand resulted in excessive measurement noise, so a $2 \times 4$-in. piece of lumber was tied to the frame of the simulator to serve as a rigid anchor when necessary.

This quantization process consisted of the following steps for each control axis:

1. Zero scale.

2. Increment the DC voltage applied across the CMND+ and CMND- terminals on the motor controller by $0.5 \mathrm{~V}$.

3. Record the force increment measured by the scale.

These steps were repeated until the applied command voltage reached the 10 VDC maximum for the motor drives. In each case, the polarity of the applied voltage was selected so that the controls would tend to rotate such that the scale was in tension. Applied voltage was controlled using the LabJack "Kipling" DAQ control program. A LabJack T7 DAQ was used in conjunction with two LJTick-DAC modules to generate 
the \pm 10 VDC command signal. Photographs from the testing process are shown in Figure 5.21.

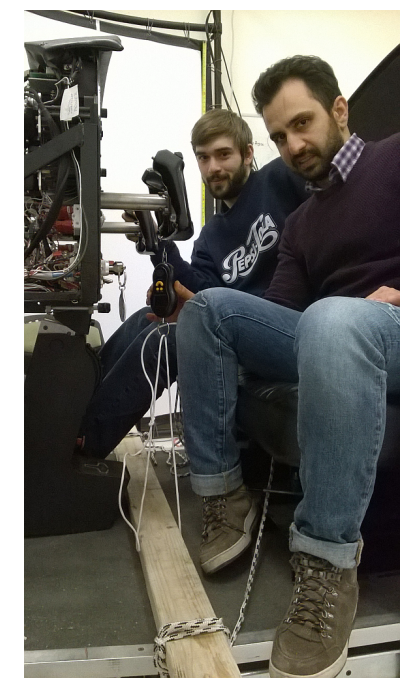

(a) Measuring aileron force. Assistants: Hooman Rajaee (front), Stefano Sandri (rear).

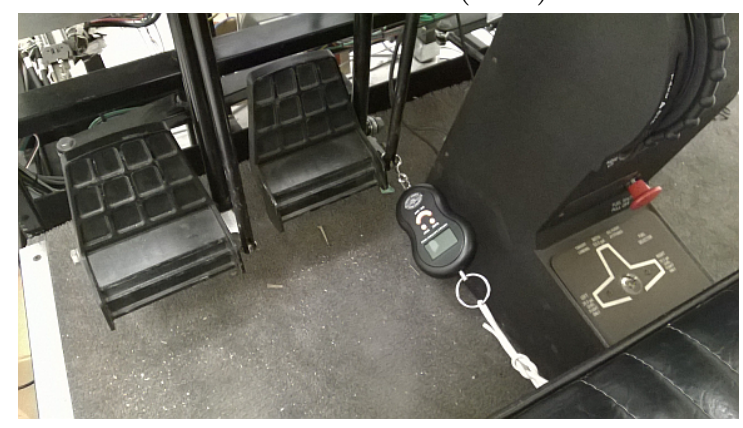

(c) Measuring rudder force. The scale is tied to the seat mount.

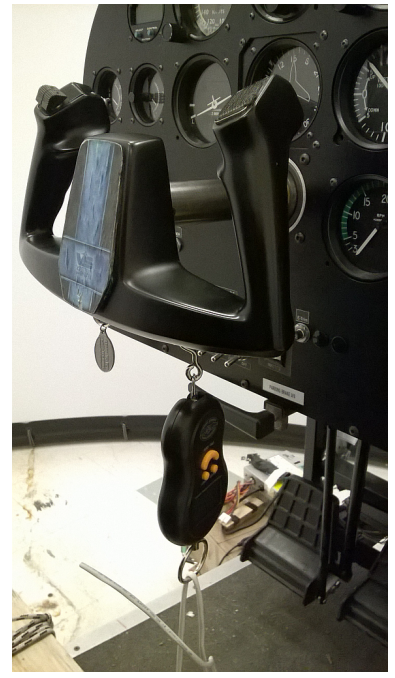

(b) Attachment of scale to yoke for aileron force measurement.

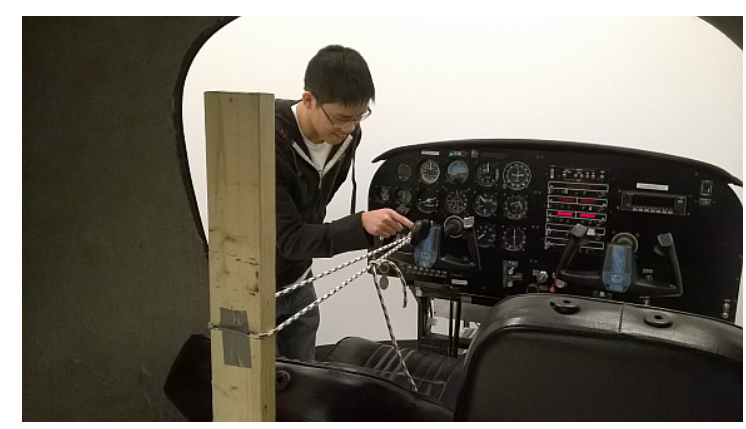

(d) Measuring elevator force. Seatback was reclined to allow access to the anchor point. Assistant: Bradley Jung.

Figure 5.21: Setup of the apparatus used to test the control loading servo drives and associated motors. Note the use of a wooden $2 \times 4$ as an anchor point for the scale for both the aileron and elevator control force measurements.

The results of the control force quantization tests are shown in Figure 5.22. In this plot, the elevator voltages have been shown as positive for plotting purposes. Due to friction in the linkages, it was difficult to accurately measure force output for small input voltages. Therefore, obviously non-linear datapoints near the origin have been 
omitted. All trendlines are linear, and have been forced to pass through the origin, since command voltage should be zero for zero output force.

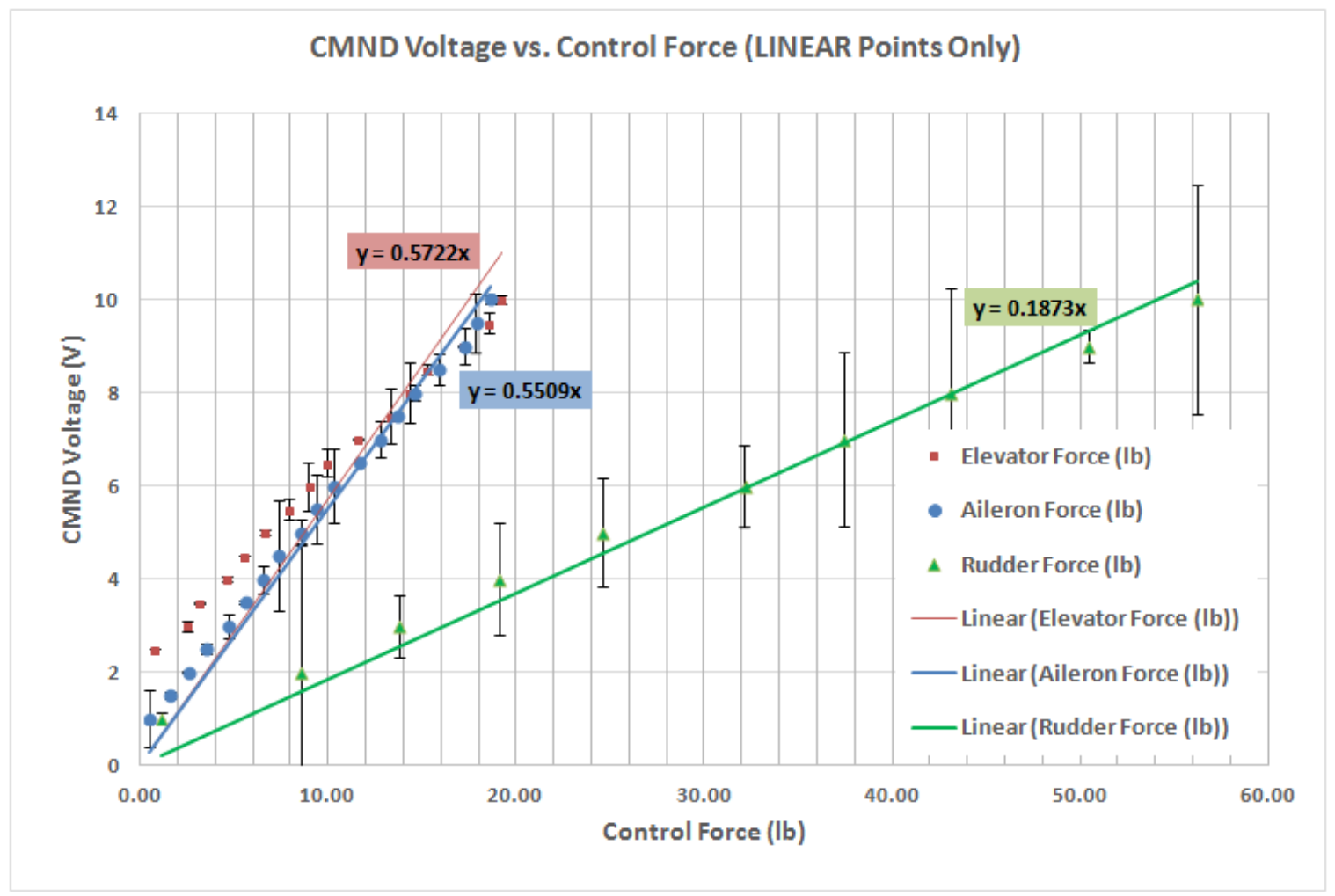

Figure 5.22: Input command voltage versus output control force for each control loading motor. Note that elevator command voltages have been shown as positive for plotting purposes. Error bars indicate \pm one standard deviation.

\subsubsection{Initial Software Interface to the Control Loading Sys- tem}

Control force depends in part on the instantaneous position of the flight controls. The positions of the flight controls were in turn measured by the analogue inputs (AINs) on a LabJack DAQ. Thus, control loading functionality was included as an integral part of the analogue input interface program detailed previously in Section 5.2.3.

The equations of the linear trendlines shown in Figure 5.22 were used to map the control force values obtained from X-Plane to the command voltages required for 
each motor to produce the corresponding output forces on the flight controls. This mapping is shown in Table 5.9.

Table 5.9: Calibration relationships between X-Plane stick forces and corresponding command voltages for the CURVS control loading system.

\begin{tabular}{|ccccc|}
\hline Command Voltage & $=$ & Slope of Trendline & $\times$ & Stick Force from X-Plane \\
\hline \hline $\mathrm{V}_{\text {Elevator }}$ & $=$ & 0.5722 & $\times$ & $\mathrm{F}_{\text {Elevator }}$ \\
\hline $\mathrm{V}_{\text {Aileron }}$ & $=$ & 0.5509 & $\times$ & $\mathrm{F}_{\text {Aileron }}$ \\
\hline $\mathrm{V}_{\text {Rudder }}$ & $=$ & 0.1873 & $\times$ & $\mathrm{F}_{\text {Rudder }}$ \\
\hline
\end{tabular}

\subsubsection{Stability Issues}

Given the right conditions, it was discovered that the redeveloped control loading system could become unstable, with the flight controls tending to oscillate back and forth instead of settling to their respective neutral (or trimmed) positions ${ }^{1}$. This problem was particularly apparent at airspeeds near or above the cruise speed (124 kts). Oscillation could be triggered by making a large control input and the suddenly releasing the controls, or by making a series of quick, repetitive control inputs. Oscillations tended to be of short period and large amplitude, with the amplitude typically increasing until the controls hit their mechanical endstops. While this divergent behaviour of the flight controls could be damped out by the pilot, its presence was detrimental both to simulator fidelity and pilot safety.

Increasing the X-Plane damping values in Plane-Maker through a trial-and-error tuning process was partially successful in reducing the oscillatory tendencies of the

\footnotetext{
${ }^{1}$ In a Cessna 172, the neutral positions of the roll and yaw controls are identical to their centre positions. The neutral position of the elevator control varies depending on the setting of the trim control. When released by the pilot, the elevator control will return to a given "neutral" position; however, this position is often different from the centre position, and depends on the particular trim setting in use at that time.
} 
flight controls. However, it was not possible to completely eliminate the problem, especially in the pitch and yaw axes. Higher values of the X-Plane damping coefficients reduced the tendency of the controls to oscillate when suddenly displaced. However, they also caused the controls to become unrealistically stiff and hard to move, as if the damping was opposing even pilot input. Oiling the joints in the linkages between the motors and the pilot flight controls exacerbated the problem, as the retarding force of friction was thereby reduced.

Compounding the stability problem was the issue of potentiometer noise. This noise manifested itself in the form of "kicks" in the control loading forces, especially at certain control positions. This indicated that the potentiometers were either dirty or worn out. These impulse-like phantom control inputs were also capable of triggering oscillation of the flight controls. In order to address this problem, the pitch, roll, and yaw potentiometers were treated with several applications of DeoxIT ${ }^{\circledR}$ Fader F5 solution by Caig Laboratories, Inc [92]. Cleaning the pots eliminated the issue of spurious control inputs and resulting kicks. After several months, however, the issue started to reappear, although not as severely as before. This indicated that the potentiometers were worn out and would require replacement in the near future.

\subsubsection{Hypothesized Causes of Stability Issues.}

The instability observed in the control loading system was hypothesized to be due to two causes:

1. Insufficient control force update rate.

2. Lack of damping.

First, the rate at which the control loading forces are updated depends on the X-Plane frame rate. This is due to the use of the NASA X-Plane Connect plugin to communicate with X-Plane, and the fact that plugins are only run by X-Plane 
once per simulation cycle [93]. Since X-Plane's rendering options were set up to give a framerate of $60 \mathrm{FPS}$, the theoretical maximum force update frequency was therefore $60 \mathrm{~Hz}$. In practice, the control force update frequency was found to be approximately $40 \mathrm{~Hz}$. In contrast, professional force-feedback systems are typically run at iteration frequencies of at least $500 \mathrm{~Hz}$, with some systems featuring rates above $4000 \mathrm{~Hz}$ [94-96].

Second, the control loading system contains no physical dampers to retard any excessive motion of the flight controls which may occur when the pilot is not actively holding the controls. Mechanical dampers generate a retarding force which is proportional to the velocity of the object to which they are connected. In CURVS, the only retarding forces present in the control loading system are due to friction in the linkages. Since friction is not typically considered to be velocity dependant, no mechanism existed to reduce (or damp) the excessive velocity of the flight controls which was observed to occur during oscillation [97].

By Newton's Second Law, the sum of the forces $F$ acting on an object is equal to the mass $m$ of the object times its resulting acceleration $a$. In mathematical form:

$$
\sum F=m \times a
$$

The summation may be expanded to show the individual forces at work on a given flight control as follows:

$$
F_{\text {motor }}+F_{\text {friction }}=m_{\text {linkages }} \times a
$$

For a large control deflection, the force of friction is negligible compared with the force exerted by the motor on the flight control. Thus, as long as stick force is applied by the control loading system, the flight control will accelerate. While stick force will decrease as the control returns to its neutral position, the flight control will 
be under continuous (although decreasing) acceleration until stick force becomes zero as the neutral position is reached. However, by that time control velocity is large and increasing, and an overshoot occurs. The process then repeats in the opposite direction, resulting in sustained oscillation.

Due to the X-Plane framerate limitation, the applied stick force is constant over each simulation frame. For simplicity of discussion, this simulation timestep will be assumed to be equal to $1 / 60^{\text {th }}$ of a second. If the pilot were to make a large control deflection, the stick force applied by the control loading system would likewise be large. If the pilot were to then release the control, the large stick force and lack of friction would cause the control to accelerate, gaining velocity as it moves back toward its neutral position. In an actual aircraft, however, the applied stick force would instantaneously decrease as the control approaches its neutral position. However, with a $1 / 60^{\text {th }}$ of a second timestep, the control algorithm is unable to adjust for the new stick forces until the next timestep. This results in a large force being applied to the yoke for the entire timestep, regardless of its current deflection. Thus, slow control loading iteration rates tend to result in excessive acceleration of the flight controls if released by the pilot after a large deflection. This makes the control loading system susceptible to oscillation. In contrast, high iteration rates will be able to reduce applied stick forces more quickly as the controls recentre, thus reducing the tendency to overshoot and oscillate.

\subsubsection{Proposed Solution to Stability Issues}

In order to raise the control loading update frequency to the recommended minimum of $500 \mathrm{~Hz}$, a new approach to control loading is required. As X-Plane can only calculate stick force data at a maximum of $60 \mathrm{~Hz}$ (the framerate of the simulation), stick forces must therefore be calculated separately from X-Plane. An external control loading algorithm lends itself well to high iteration rates based on instantaneous stick 
deflection as measured by the control potentiometers. Airspeed updates would be obtained from X-Plane once per frame in order to maintain synchronization with the simulation. Between updates, constant airspeed would be assumed, and a set number of force calculations would be performed. Since a Cessna 172 is a relatively lowperformance aircraft, it is reasonable to assume that the change in airspeed between frames will be minimal. Assuming stick force depends solely on instantaneous control deflection between X-Plane updates will allow the control loading algorithm to react more quickly to high control deflection rates, and reduce stick force more quickly. This should help to prevent overshooting and oscillation about the neutral position.

If ten force iterations based on instantaneous stick position were to be performed externally between X-Plane updates which occur every 1/60 sec., the overall iteration rate could be increased to $660 \mathrm{~Hz}$. This exceeds the minimum value of $500 \mathrm{~Hz}$ recommended in the literature.

In the event that a faster control force iteration rate is unable to stabilize the control loading system, software damping should be implemented as part of the external control loading algorithm. Damping should only be activated above an appropriate threshold value, however. The purpose of the threshold is to prevent the damping component of the algorithm from interfering with the pilot's normal, low-speed movement of the controls. This rate-trigged damping scheme is illustrated in Figure 5.23. As numerical differentiation is prone to measurement noise, position feedback from the potentiometers should first be processed with a low-pass filter (LPF) to remove high-frequency noise spikes. 


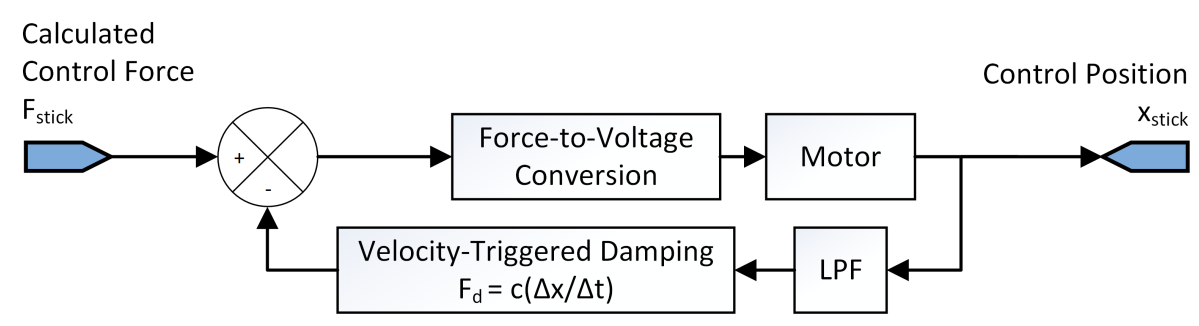

Figure 5.23: Proposed control loading algorithm with rate-triggered damping. Variable $c$ is the damping coefficient.

\subsection{Servo-based Instruments}

Most of the simulated flight instruments in the VectorSim were found to be driven by servomotors of the same type as are commonly used in radio-controlled model aircraft. A servomotor is an electric motor which incorporates a closed-loop feedback control system to allow precise angular positioning. Hobby servomotors are commonly referred to simply as servos. Position feedback is provided by a rotary potentiometer attached to the internal gear train. A representative servo taken from the fuel flow instrument in the VectorSim is shown in Figure 5.24.

Servos have three connections: power, ground, and signal. Rotational position of a servo depends on the width of the electrical pulse applied to the signal line, with the nominal "centre" position corresponding to a pulse width of approximately 1.5 milliseconds (ms) [98]. Command pulses are repeated at constant intervals of approximately $20 \mathrm{~ms}$ for a pulse repetition frequency of about $50 \mathrm{~Hz}$. Changing the command pulsewidth from $1 \mathrm{~ms}$ to $2 \mathrm{~ms}$ typically results in a rotational sweep of approximately $90^{\circ}$; however, servos can be driven further by either increasing or decreasing the pulsewidth beyond this range. The preceding information applies in general, as no official standard for hobby servomotors exists.

Example oscilloscope traces for two different servo signals are shown in Figure 5.25. These traces were obtained by sampling the output signals from an Arduino UNO microcontroller. Servo positions of $0^{\circ}$ and $180^{\circ}$ were specified using the 


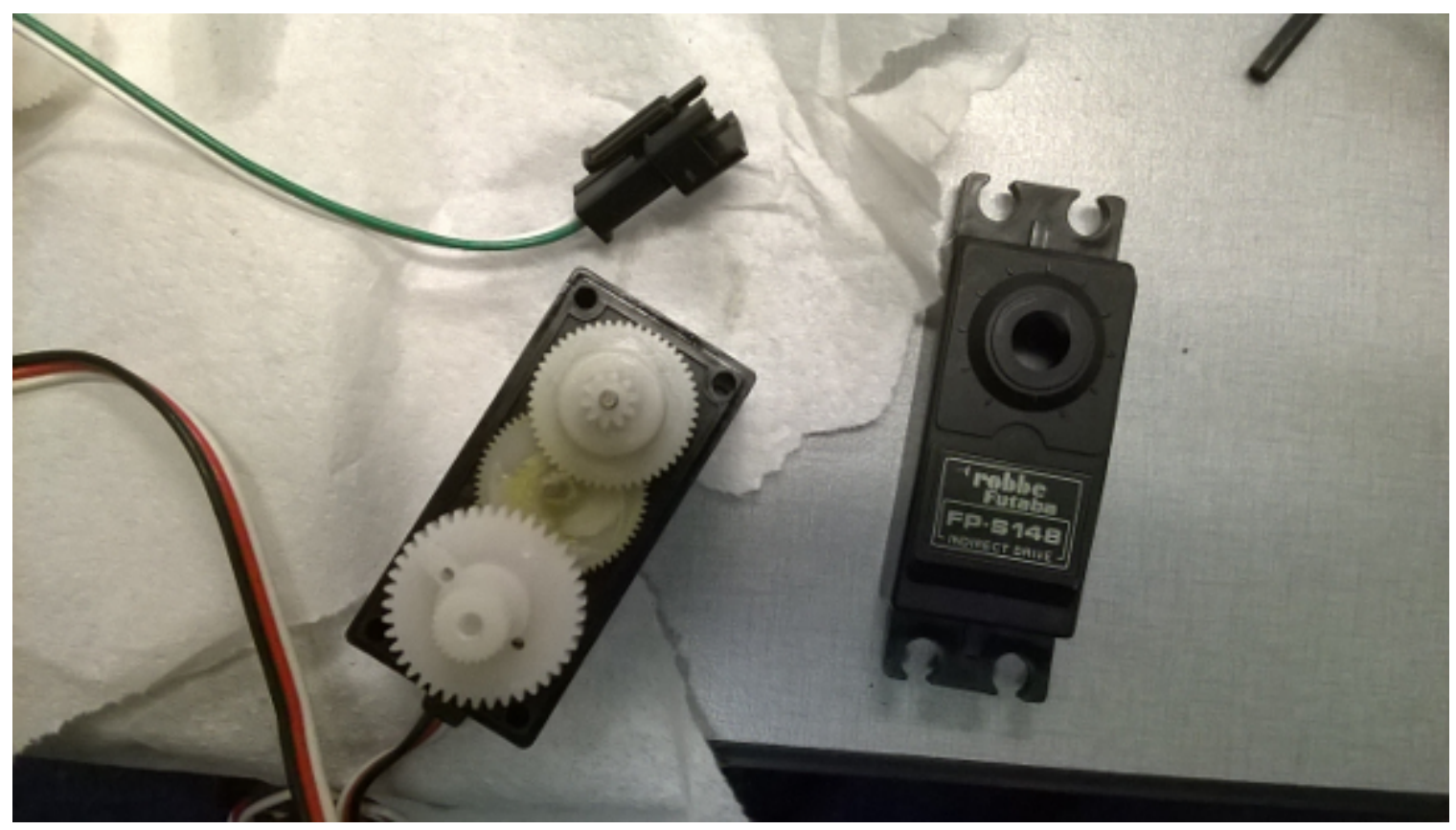

Figure 5.24: Example VectorSim servo, disassembled to show gear train. This particular servo was replaced due to stripped gears.

".write(numberOfDegrees)" command from the Arduino servo library.

\subsubsection{Reverse-Engineering the Existing Servo Instruments}

As stated previously, servo command pulse width determines the angle to which a servo will rotate. However, at some point, either the internal endstops within the servo or the instrument in which it is installed will be encountered. In order to avoid damage to the internal servo gear train (which consisted of nylon gears), it was necessary to know the positions of the endstops in terms of the corresponding command pulsewidths. An example of the physical endstops built into the servo instruments in the VectorSim is shown in Figure 5.26.

Since the VectorSim was still functional enough to initialize all of its gauges upon login to the legacy IOS program, it was decided to use this functionality to determine the initial starting pulsewidth being fed to each instrument. By starting with a 


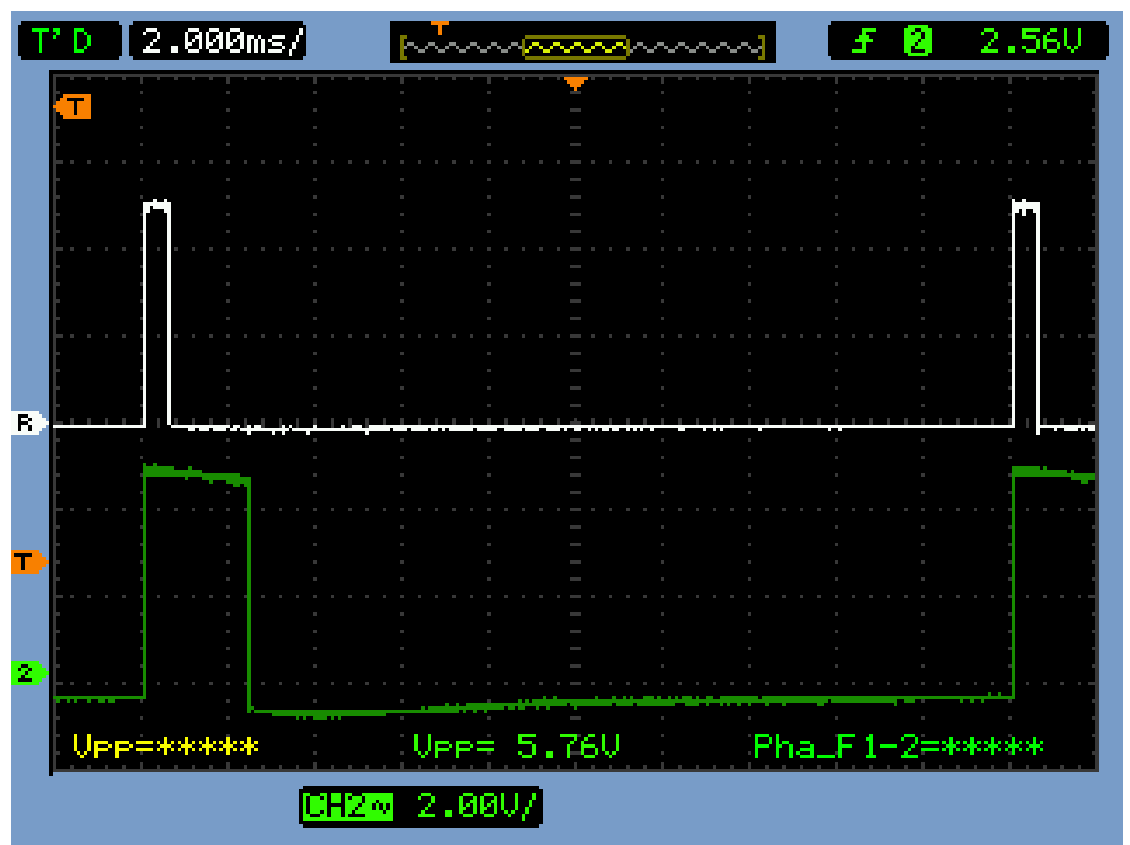

Figure 5.25: Example servo signal traces. The upper trace commands a generic servo to rotate to the $0^{\circ}$ position, while the lower trace commands a position of $180^{\circ}$. Each horizontal division represents $2.000 \mathrm{~ms}$.

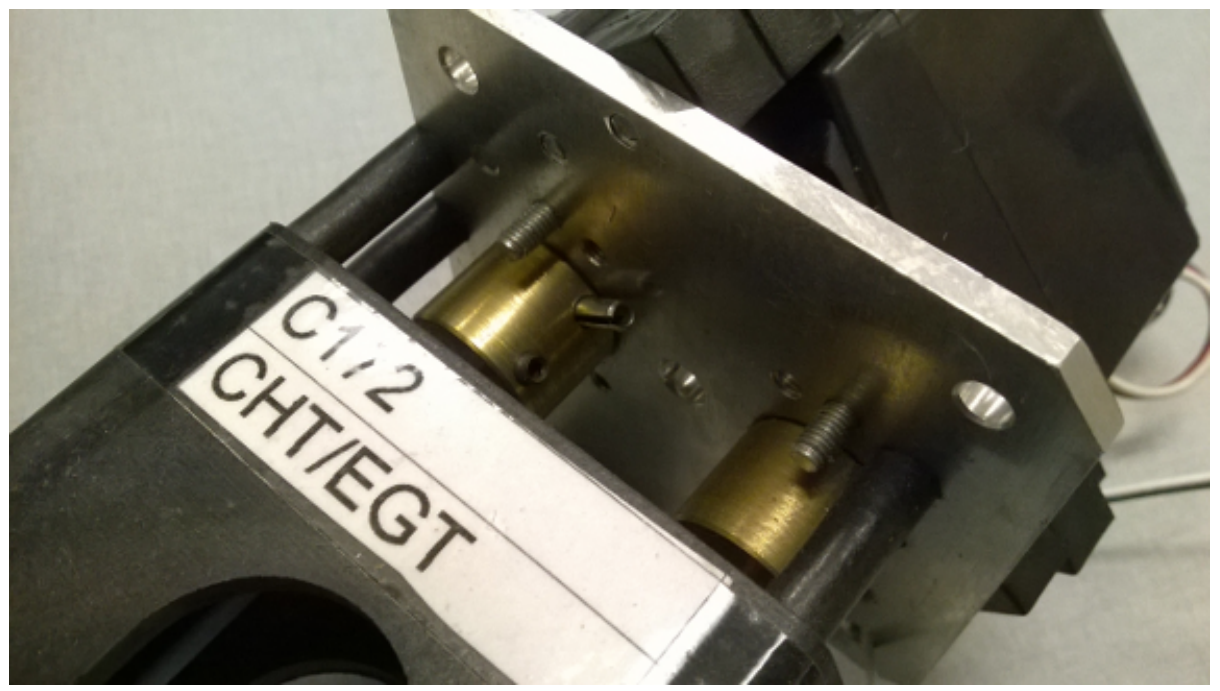

Figure 5.26: Example of a physical endstops in one of the original servo-based instruments in the VectorSim. Contact with the endstops must be avoided to prevent servo damage. 
known-good servo position command, each servo could then be carefully commanded to rotate until either gentle contact with an endstop was obtained, or the end of the instrument's calibrated range was encountered. While a servo position near the centre of range of a typical servo could have been arbitrarily applied, then experimentally adjusted in order to "feel out" the actual range of the instrument, doing so would have risked forcibly driving the servo against an instrument endstop, potentially resulting in stripped gears.

After power-up and initialization of the VectorSim under command of its original IOS software, each gauge was disconnected, and the signal line from the transputer I/O rack probed with a digital oscilloscope to record the initial command pulsewidths being fed to the instrument. Example oscilloscope traces for the airspeed indicator are shown in Figure 5.27.

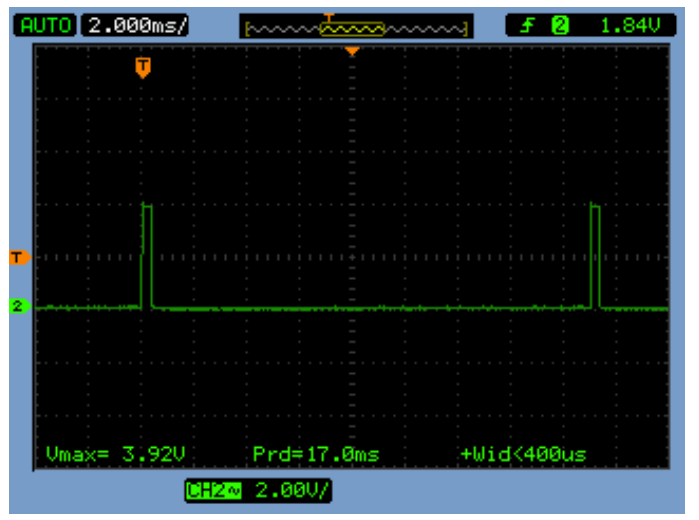

(a) Airspeed servo pulse train. Note the measured pulse-repetition period of $17 \mathrm{~ms}$.

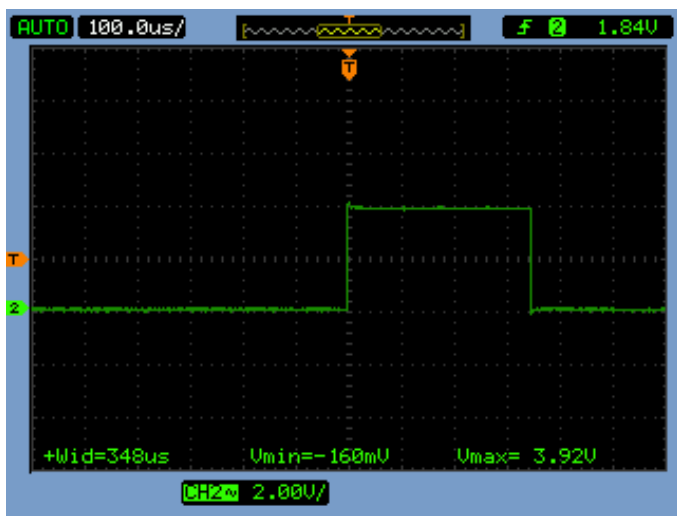

(b) Magnified view of a single pulse. Note the measured pulsewidth of 348 us (0.348 ms).

Figure 5.27: Airspeed indicator servo pulses for the initial position of the instrument (0 kts indicated airspeed).

\subsubsection{Initial Servo Instrument Interface}

In total, there were 18 servos to be controlled simultaneously. These 18 servos were divided between 10 simulated instruments, with some instruments containing up to 
three servos each. Instead of attempting to control the servos individually, the Mini Maestro 24-Channel servo controller (49.95 USD) by Pololu Corporation was selected for this task [99]. Pololu provides a USB SDK for interfacing to this device which is written in the C\# language [100]. The initial servo interface was based on the BeagleBone Black single-board Linux computer and the aforementioned Mini Maestro servo controller, as illustrated in Figure 5.28. A short, 3-ft. length of USB cable was used to connect the Mini Maestro to a BeagleBone Black single-board Linux computer, which was in turn connected to the X-Plane LAN by an Ethernet cable. Use of a USB connection was acceptable in this case as the cable length required was well below the 16-ft. maximum for USB [44].

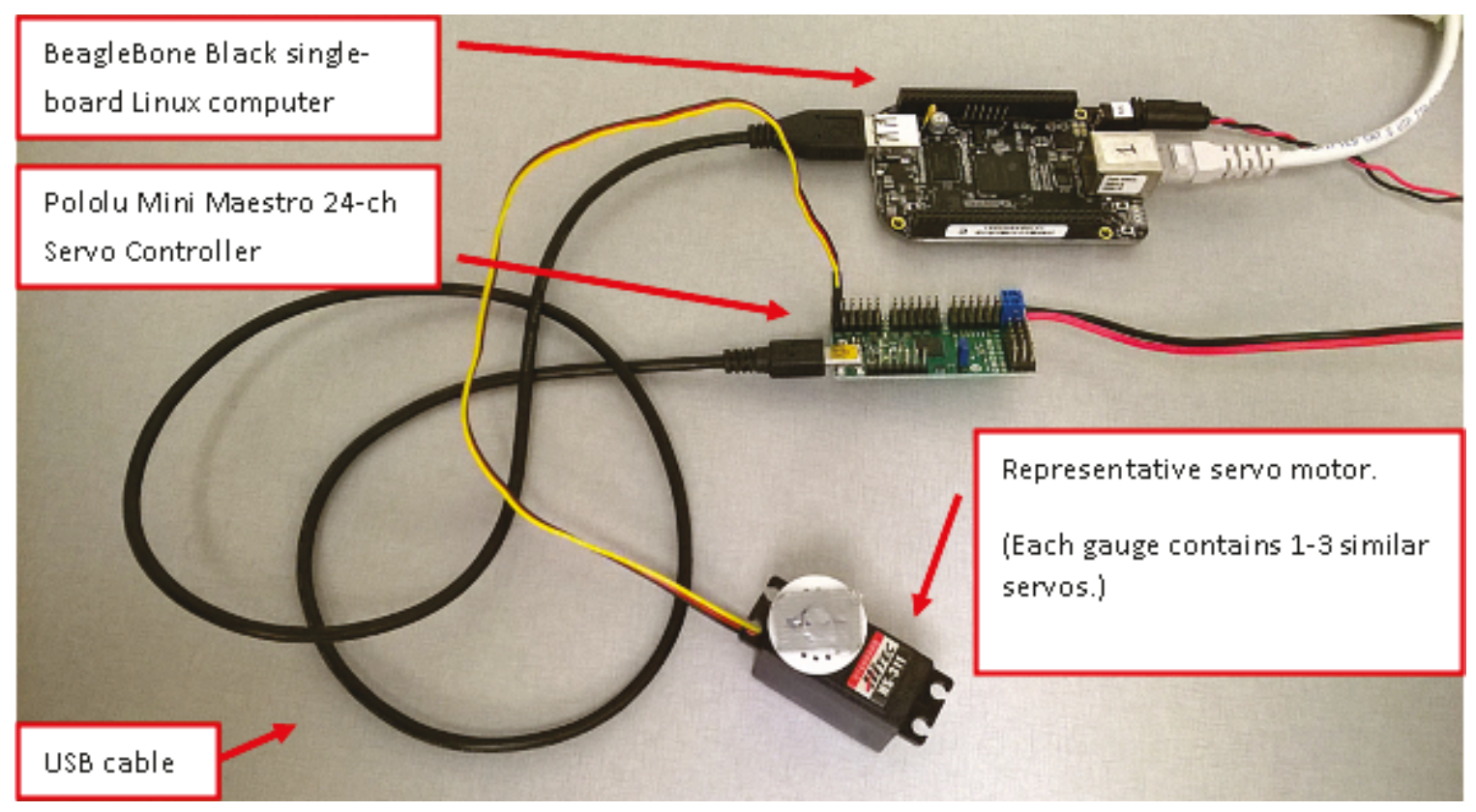

Figure 5.28: Servo control using the BeagleBone Black and Pololu Mini Maestro servo controller. The red and black wires are for supply power.

The $\mathrm{C} \#$ programming language was used in order to take advantage of the $\mathrm{C} \#$ USB functionality provided in the Pololu SDK. Since programs written in the C\# language are compiled just-in-time (JIT) at runtime, the Mono C\# compiler for Linux was required. Mono is an open-source implementation of the Windows-specific .NET 
Framework by Microsoft [101]. In order to install Mono to the BBB, however, the Debian Linux operating system which shipped with the device had to be upgraded to a Debian version which supported floating-point mathematics in hardware, as opposed to software ("armhf" versus "armel") [102]. The cross-platform compatibility of $\mathrm{C} \#$ allowed the instrument interface code to be developed and tested on a fast, Windows 7 PC using the Microsoft Visual Studio Express 2013 integrated development environment (IDE), then deployed on the slower BBB for actual use. The only change required was the use of different Pololu libraries for Windows or Linux. This code portability is in contrast to compiled languages such as $\mathrm{C}++$, which must be compiled specifically for the exact computer architecture which will be used to run the finished program.

Simulation data to drive the connected servo instruments was initially obtained by selecting UDP datasets for output from within the X-Plane software, as shown in Figure 5.29. This caused X-Plane to transmit UDP packets over the simulation LAN to the IP address of the BBB. A complete listing of the X-Plane UDP datasets and the individual variables available within each is given in Appendix D.

Each packet ("datagram") contains the selected datasets in ascending numerical order, as described in detail in Appendix C. This approach was found to be susceptible to error, however, as the UDP data output settings are easily accessible to the enduser, and the ordering of the datasets within a packet depends on which particular datasets are selected. Thus, the inadvertent selection or de-selection of datasets can easily result a re-ordering of the contents of the datagram. This in turn can cause incorrect information to be fed to the simulated instruments. While it would have been possible to overcome this issue by parsing the contents of each datagram based on dataset number instead of position within the datagram, it also became apparent that the UDP datasets almost always provided actual values, not the indicated values presented to the pilot in the cockpit. This shortcoming meant that instrument failures 


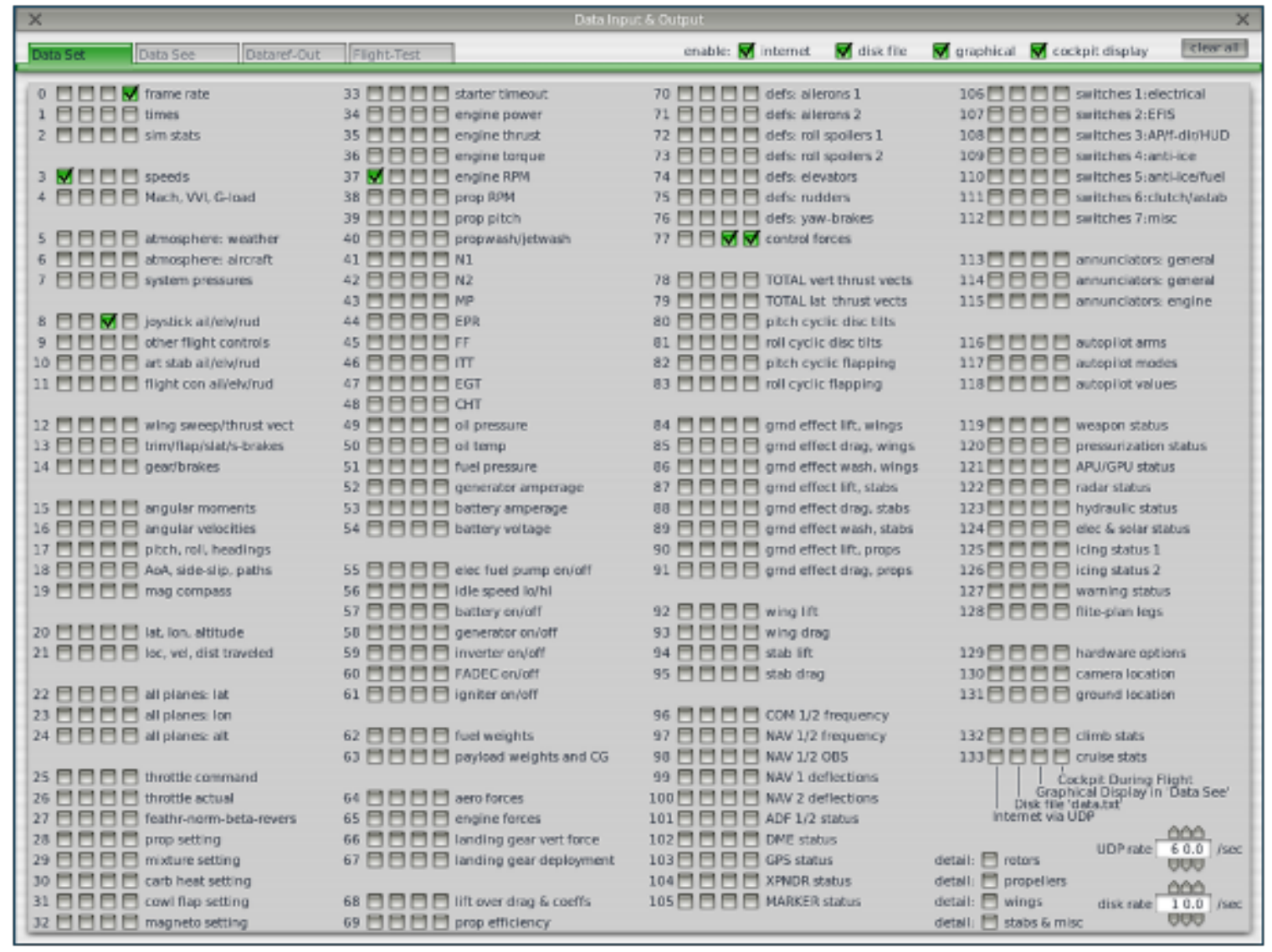

Figure 5.29: X-Plane UDP dataset selection. Datasets \#3 ("speeds") and \#37 ("engine RPM") are shown selected for UDP ("internet") output at a rate of 60 packets per second. 
would be impossible to simulate if the servo instruments were to be driven from the X-Plane UDP datasets.

Simulation of instrument failures is not required by Transport Canada for certification as a Level 2 FTD; however, this ability is commonly expected to be present in a modern FTD. In addition, it was discovered that one critical piece of information the deflection of the skid-and-slip ball in the turn coordinator - is not included in the UDP datasets. While it would have been possible to overcome these two issues by programming additional functionality into the instrument interface code, this would have greatly increased project scope and complexity, and resulted in the duplication of existing X-Plane functionality. Thus a different approach was required, one which would be able to access the full complement of datarefs available from X-Plane.

\subsubsection{Final Servo Instrument Interface}

Pursuant to the revised system architecture detailed in Section 4.4, the BeagleBone Black was removed from the final servo instrument interface system. As the primary

purpose of the BBB had been to provide an Ethernet interface to the USB-based servo controller, a replacement for this functionality was required. Further research revealed two potential solutions to this problem, detailed below:

- Active USB extension cables

- USB range extenders

Active USB extension cables include amplification circuitry at the remote end. This amplification circuitry is typically powered by the $+5 \mathrm{VDC}$ provided by the USB port on the host PC. In contrast, USB range extenders make use of interface devices at both the $\mathrm{PC}$ and remote endpoints, connected by an Ethernet cable. Due to the use of Ethernet elsewhere in CURVS, a low-cost (18.54 CAD) USB range extender was selected for evaluation when used in conjunction with a $30 \mathrm{ft}$. CAT6 Ethernet 
cable. Shown in Figure 5.30, this unit is available for mail order under different brand names from a variety of online retailers. The particular unit evaluated was the "USB RJ45 Extension Adapter" from Monoprice, Inc. [103].

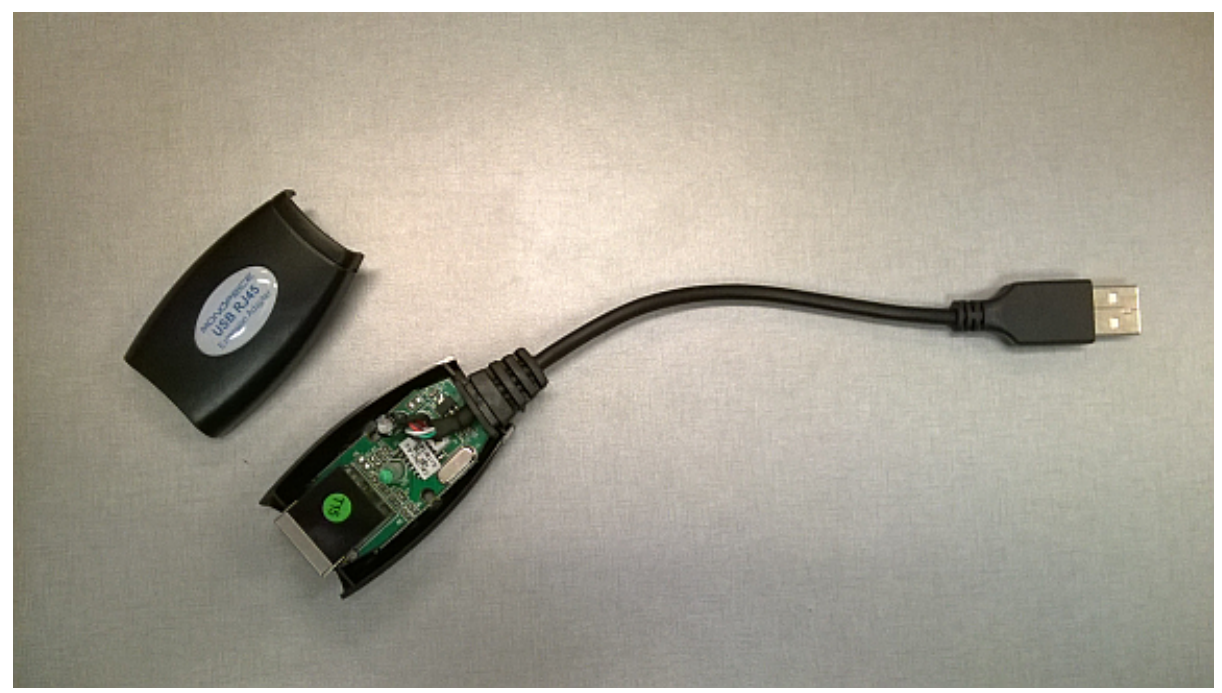

Figure 5.30: Low-cost USB range extender for the PC endpoint. Cover has been removed to examine the internal circuitry. A corresponding unit is required at the remote endpoint.

Use of the USB range extender enabled the C\# servo instrument interface program to be run on the master PC instead of the Beaglebone Black. Thus, it was possible to use the USB-based Mini Maestro servo controller at a much greater distance from the master PC than would have been ordinarily permitted by the USB standard.

In order to access the X-Plane datarefs containing the indicated values of the flight instruments, it was decided to port the servo instrument interface program from $\mathrm{C \#}$ to $\mathrm{C}++$. This change in language allowed use of the $\mathrm{C}++$-based NASA X-Plane Connect toobox, thus enabling access to the complete set of X-Plane datarefs. The use of $\mathrm{C}++$ also allowed reuse of previously-written $\mathrm{C}++$ code to enable the readingin of CSV calibration files. Changing to $\mathrm{C}++$ also meant abandoning the use of the C\# USB communication methods contained in the Pololu USB SDK in favour of direct, byte-level communication with the Mini Maestro using a virtual serial port as 
per the example contained in the Pololu Maestro Servo Controller User's Guide [104].

A high-level overview of the program flow within the final version of the CURVS servo instrument interface program is shown schematically in Figure 5.31. The "instrument update" sub-process is shown separately in Figure 5.32.

Interfacing the physical gauges in the original VectorSim cockpit to X-Plane was complicated by the fact that the scales on many of the instruments were non-linear. For example, referring to the airspeed indicator shown in Figure 5.33, it may be seen that the spacing between the calibration markings decreased as indicated airspeed increased.

The non-linearity of the airspeed indicator is shown graphically in Figure 5.34. This plot was generated by positioning the instrument needle at the desired indicated airspeed values, then noting the corresponding pulsewidth value being fed to the servo.

In the original VectorSim, six airspeed-pulsewidth calibration points had been chosen across the range of the instrument; however, in testing, it was found that this original scheme caused the airspeed indicator to read low in the range $80 \mathrm{kts}-120$ kts. Thus, four additional calibration points were added to increase the accuracy of the instrument. The old and new calibration points are compared in Table 5.10. In continued testing, the airspeed indicator was found to read low at approximately 180 kts; however, as this is significantly above the never-exceed (redline) speed of $163 \mathrm{kts}$ for a Cessna 172, no attempt was made to correct this error [88].

To determine the pulsewidth which corresponded to a given value of indicated airspeed from X-Plane, the CURVS servo instrument interface program performed linear interpolation between the appropriate set of calibration points. A similar process was also performed for each of the other servo-driven flight instruments.

For instruments which were designed to be able to indicate beyond their calibrated range, an additional sanity check was performed post-interpolation to ensure that the 


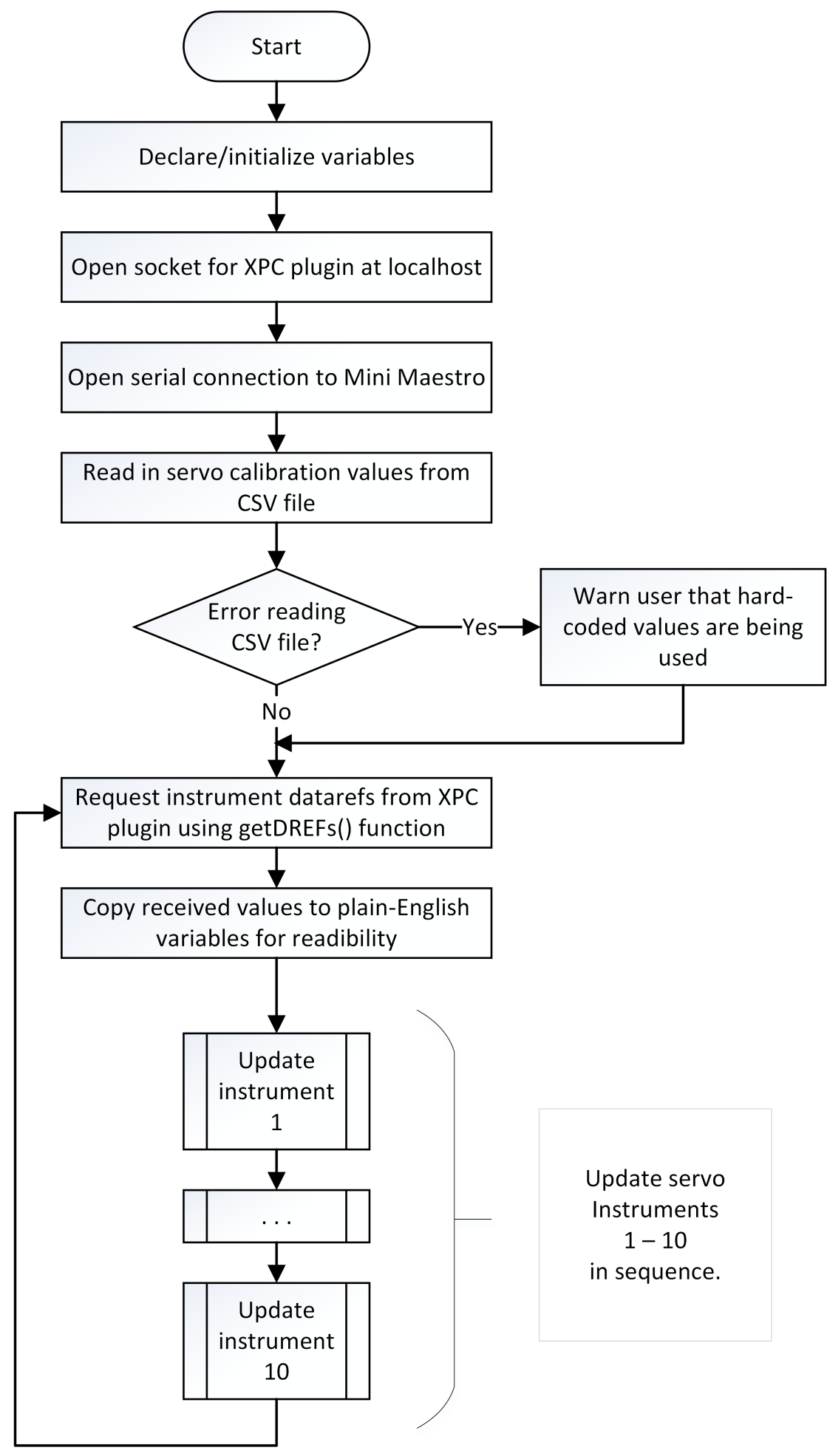

Figure 5.31: CURVS servo instrument interface program flowchart. Note that the "update instrument" sub-process is detailed in Figure 5.32. 


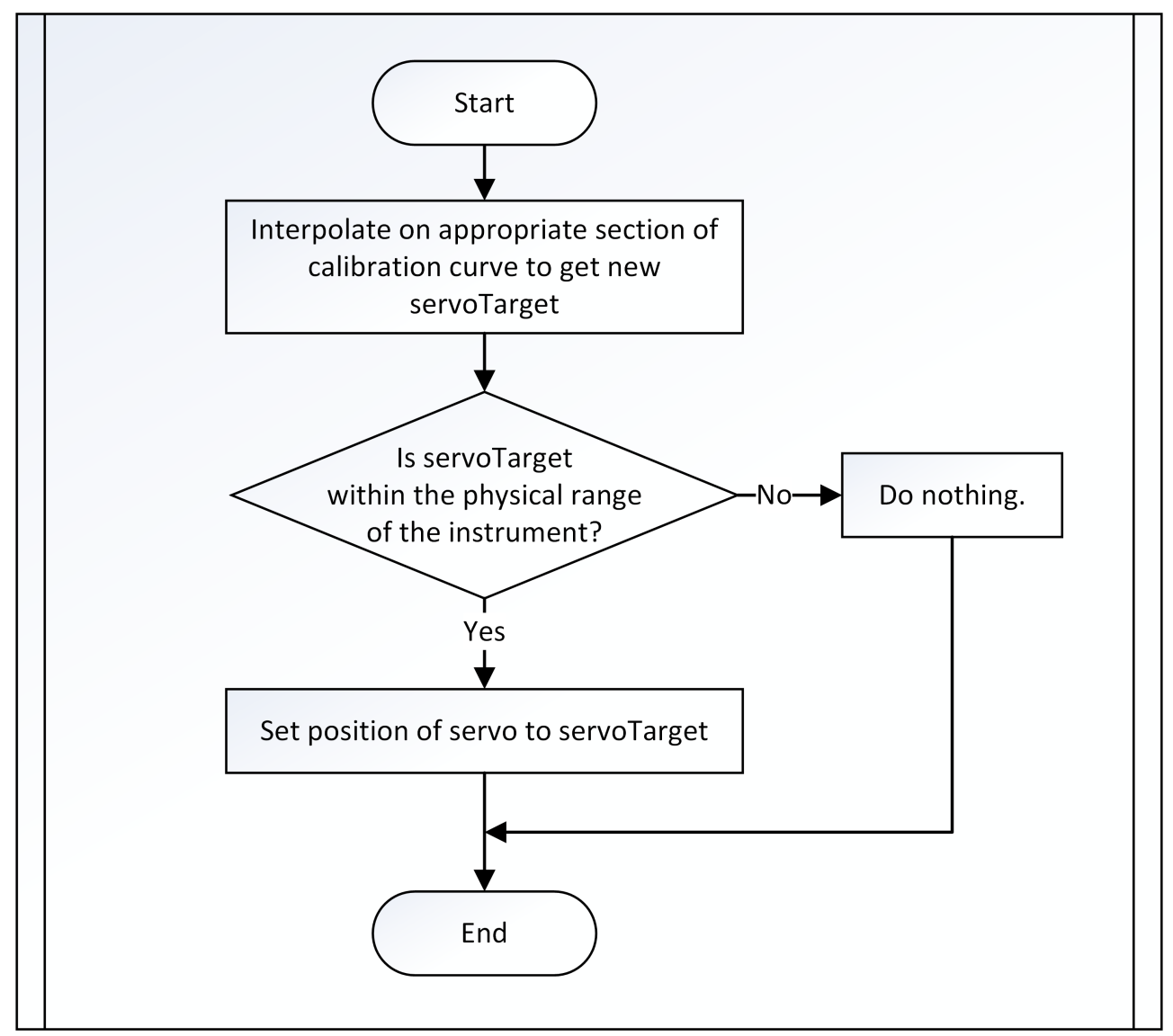

Figure 5.32: Details of the CURVS "update instrument" sub-process.

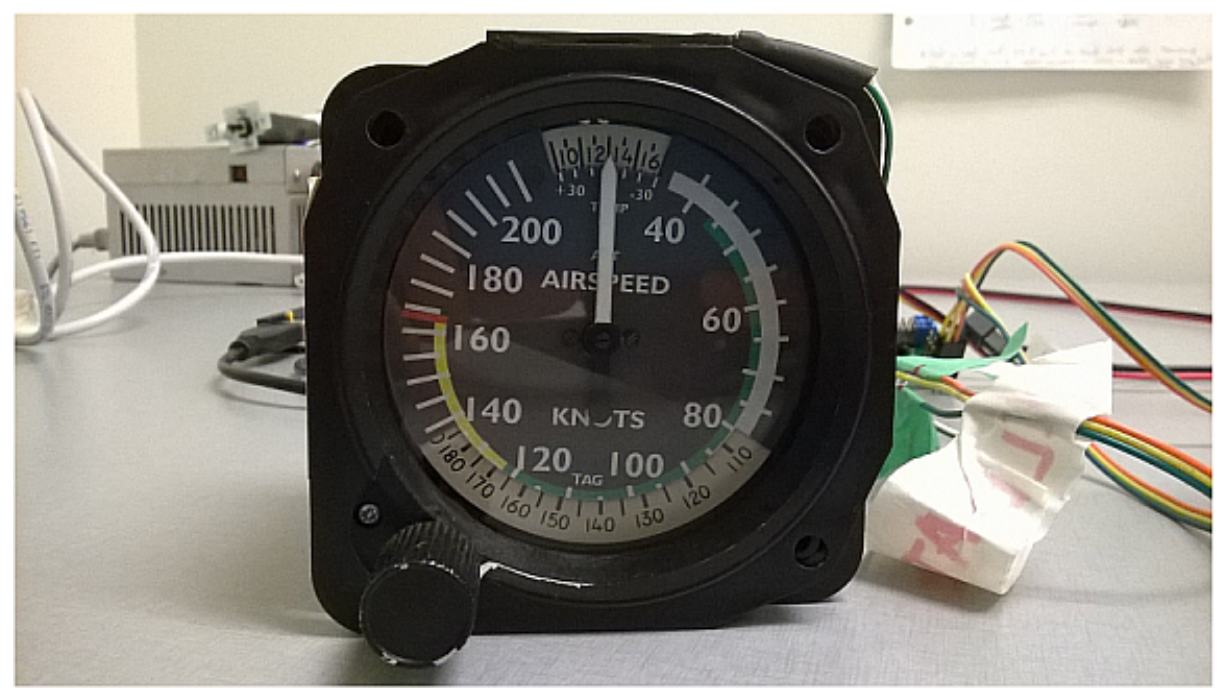

Figure 5.33: VectorSim servo-driven airspeed indicator. Note that the spacing between calibration marks is not constant. 


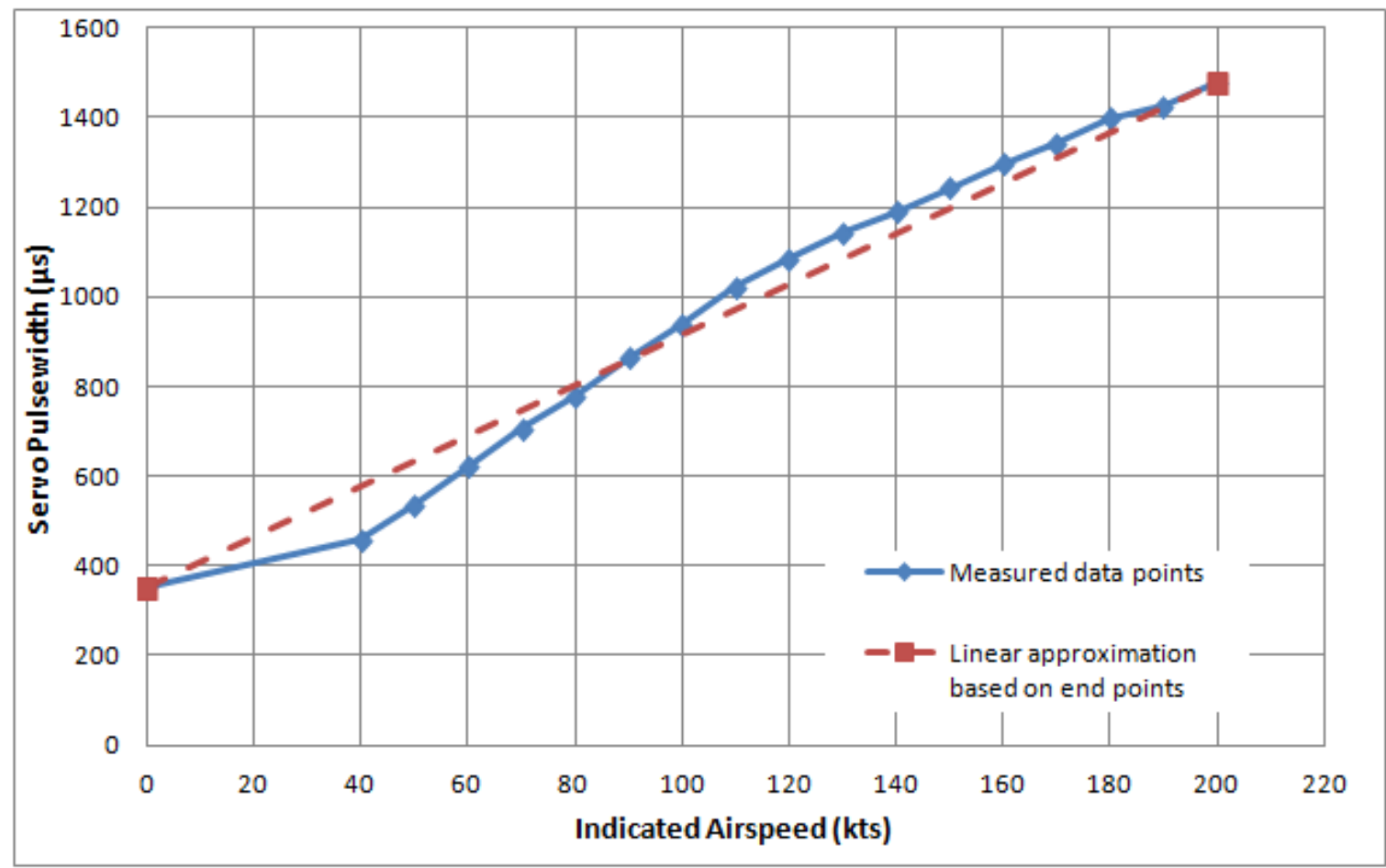

Figure 5.34: The non-linearity of the airspeed indicator is shown by a plot of indicated airspeed (kts) against the corresponding servo pulsewidth ( $\mu \mathrm{s})$. The dashed line represents a linear approximation using only the start and end points, while the solid curve represents data points taken every $10 \mathrm{kts}$ across the calibrated range of the instrument.

Table 5.10: Comparison of original vs. new servo calibration points for the airspeed indicator. Calibration points consist of an indicated airspeed and the corresponding servo command pulsewidth.

\begin{tabular}{|l|c|c|c|c|c|c|c|c|c|c|}
\hline & \multicolumn{10}{|c|}{ Calibration Points } \\
\hline Original (kts) & 0 & 40 & 70 & & & 100 & & 120 & 160 & \\
\hline New (kts) & 0 & 40 & 70 & 80 & 90 & 100 & 110 & 120 & 160 & 200 \\
\hline \hline Pulsewidth (us) & 352 & 459 & 707 & 780 & 864 & 906 & 1022 & 1087 & 1297 & 1478 \\
\hline
\end{tabular}


calculated servo pulsewidth was within the physical range of the instrument. This prevented collisions with the physical endstops built into each of the servo instruments.

\subsection{Stepper-based Instruments}

Due to their need for continuous rotation, the magnetic compass, directional gyro, automatic direction finder $(\mathrm{ADF})$, and altimeter were driven by stepper motors instead of servos. A stepper motor is capable of unbounded rotation, albeit only in discrete steps. Each step is typically a fraction of a degree. The stepper motors used in the simulated altimeter, directional gyro, and ADF were manufactured by Japan Servo Co. and were capable of $0.9^{\circ} /$ step. The stepper used in the simulated compass did not have a label; however, it appeared to be visually identical to the other steppers. It was not possible to obtain a datasheet for these stepper motors as the original manufacturer had since been acquired by Nidec Servo Corporation, who indicated that the VectorSim steppers were custom products and protected under a non-disclosure agreement. Given the limited number of simulators produced by VTS, it is extremely unlikely VTS commissioned the custom manufacture of any stepper motors; however, it is possible that they were acquired as surplus from another project.

In the absence of an official datasheet, the stepper motors were reverse engineered. The stepper motors were determined to be of permanent magnet (PM) type, as individual "steps" due to the magnetic rotor could be felt when rotating the motor shaft by hand [105]. An example of a simple two-pole PM stepper motor is shown in Figure 5.35.

Each motor contained four terminals connected together in pairs, one pair for each coil. This indicated a bipolar stepper motor [106], a schematic for which is shown in Figure 5.36. In a bipolar stepper, the direction of current flow through each coil 


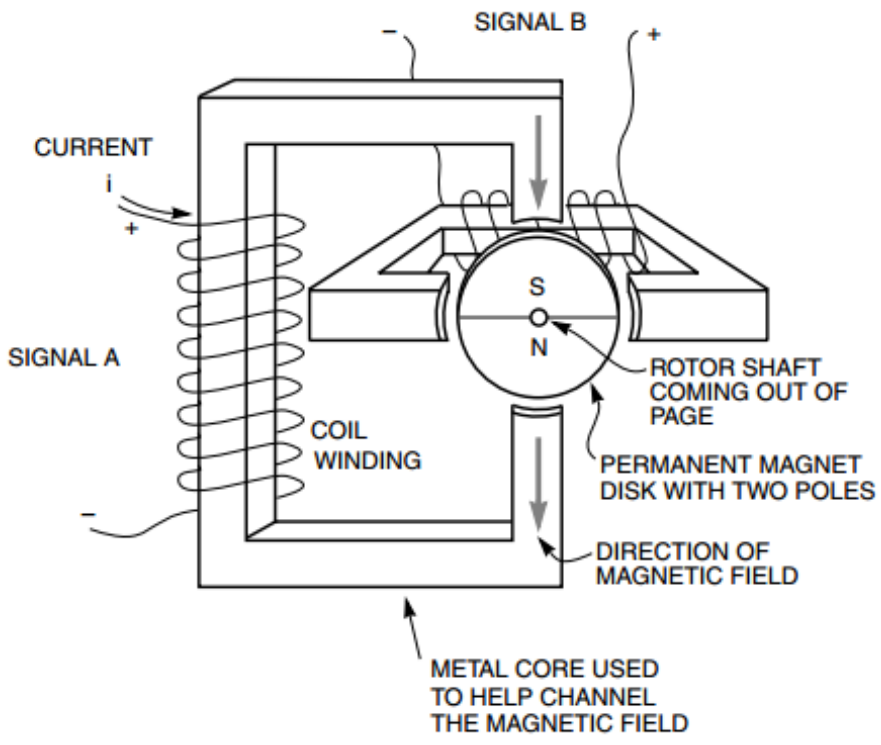

Figure 5.35: Example of a simple 2-pole permanent-magnet stepper motor. Image from [105].

is reversible, allowing each coil to assume two distinct polarities. The resistance of each coil was measured to be $41 \Omega$. The VectorSim supply power to the steppers was measured to be 12 VDC. Quiescent current draw was measured at $250 \mathrm{~mA}$ per coil.

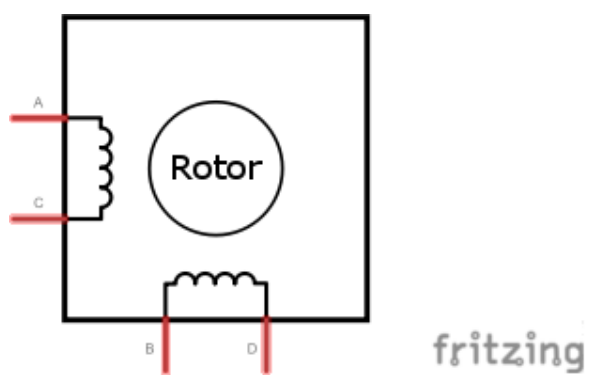

Figure 5.36: Bipolar stepper motor schematic.

Due to the special sequence in which the motor coils must be pulsed in order to cause rotation, stepper motors are usually driven by stepper motor driver ICs. The VectorSim instruments were driven by Motorola MC3479 stepper motor driver ICs located on the KATIF-1 board. The datasheet for the stepper motor drivers was located, and the drivers were found to be capable of either full or half-step operation 
while supplying up to $350 \mathrm{~mA}$ per coil [107]. In half-step operation, the motor driver changes the pulse sequence sent to the coils, doubling of the number of steps per revolution, and thus increasing the angular resolution. Probing Pin 9 (the full/half step selector) on the motor driver IC with a digital voltmeter revealed that the pin was being driven HIGH by KATIF-1, indicating that the steppers were being operated in half-step mode.

Since stepper motors provide no position feedback, a Honeywell HOA2001 transmissive Optoschmitt sensor was used in conjunction with a slotted disc for this purpose, as shown in Figure 5.37. According to the datasheet, the output from the HOA2001 is LOW when the light signal is blocked, and HIGH when it is not [108]. This arrangement was used on the simulated altimeter, magnetic compass, and ADF. The directional gyro required no position feedback, as the reading from this instrument is relative to its starting value. This starting value is set manually by the pilot to match the reading on the magnetic compass before takeoff. Due to mechanical friction, the indication on an actual directional gyro will drift in flight, requiring manual calibration by the pilot at regular intervals.

The use of a slotted disc and light sensor allowed the stepper-based instruments to be automatically initialized to a known starting position. During a simulation session, the simulated instruments could then be continuously updated based on the difference between the known previous position and the new value. In order to interface the stepper-based instruments to X-Plane via the LabJack T7 DAQ, a $\mathrm{C}++$ interface program was required. The required program logic for a single stepper-based instrument with position feedback was represented in the form of a flowchart, as shown in Figure 5.38. 


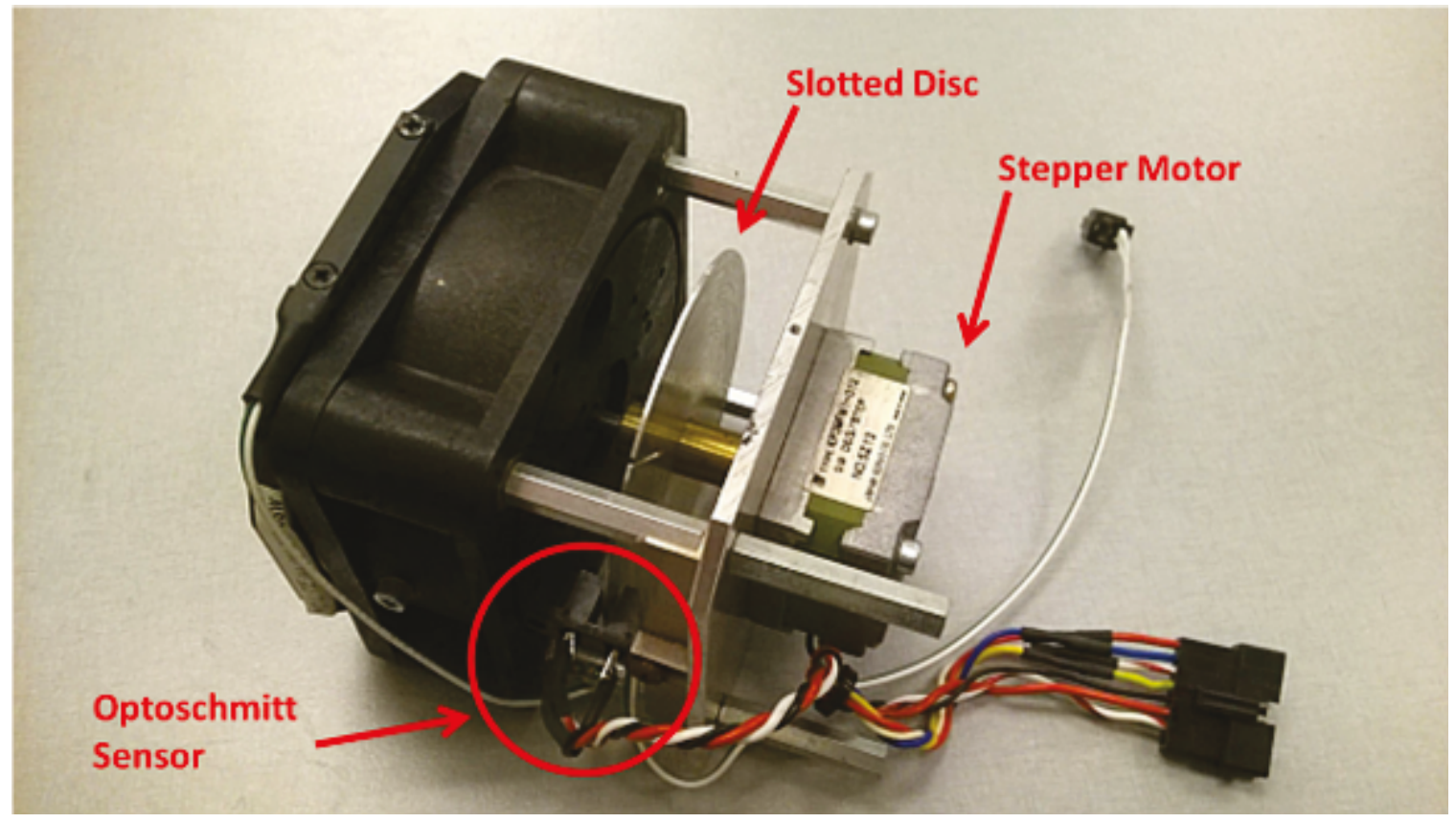

Figure 5.37: Slotted-disc and Optoschmitt sensor on the ADF. 


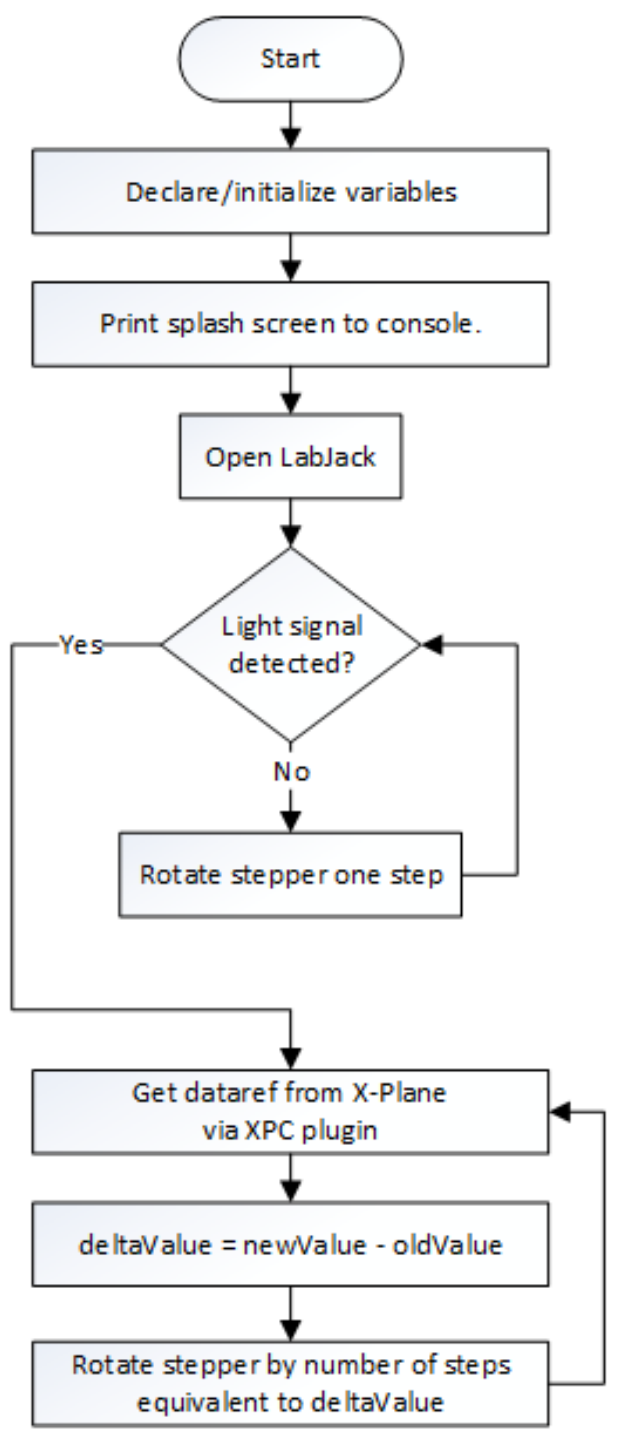

Figure 5.38: Stepper motor instrument interface flowchart. 


\subsection{GPS}

The advent of the American global positioning system (GPS) and associated satellite constellation has revolutionized aerial navigation. While all modern pilots are still trained how to navigate by map and compass, the use of the GPS system is fast becoming ubiquitous. The original VectorSim as received from WWFC was equipped with a dated Bendix-King KLN-89B GPS unit, shown in Figure 5.39. As WWFC is transitioning away from the use of this legacy device, it was decided to upgrade the simulator to use a more modern GPS. The search for and implementation of a replacement GPS was delegated to project assistant Karim Youssef, a third-year aerospace engineering undergraduate student at Carleton University working under the direction of the author.

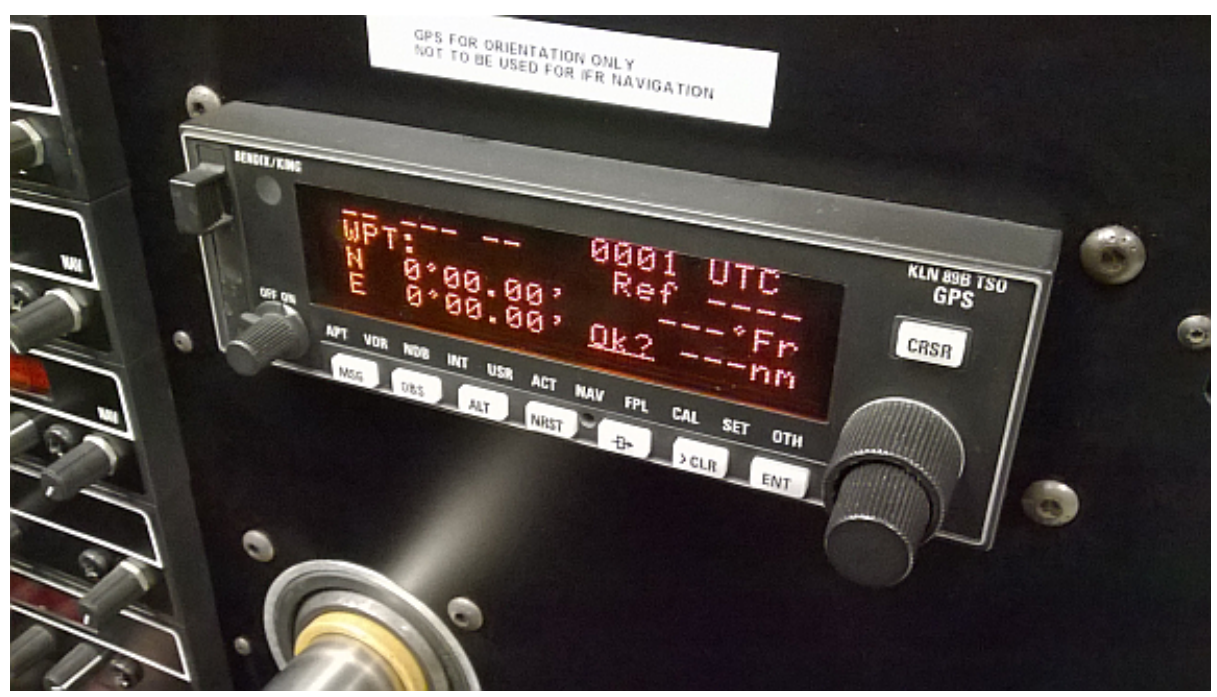

Figure 5.39: The original Bendix-King KLN89B GPS.

As of this writing, use of the Garmin GNS 430/430W GPS receiver in general aviation is widespread. In addition to its GPS features, the GNS 430/430W is also capable of acting as a NAV/COM radio, as well as being interfaced to a course deviation indicator (CDI). Initially, it was thought that an actual GNS430 could be interfaced 
to X-Plane. Other Garmin GPS units include a "simulator mode," in which coordinates may be fed to the device from an external source, causing the device to behave functionally as if it were in motion. However, the stock GNS430 does not include a simulator mode. Instead a specially-manufactured simulator version must be used. X-Plane is capable of natively driving such simulated Garmin GNS430 units used in conjunction with a professional-use licence key. A simulator version of the GNS430 was formerly available from Garmin, however, it has since been discontinued. Used units were found to be available, but at a cost significantly above the cost of a fullyfunctional unit. A simulated version of the Garmin 430W, which included support for the Wide Area Augmentation System (WAAS), was never released. As purchase of a used simulator version of the GNS 430 unit would have cost approximately 10,000 CAD, consuming approximately $1 / 3$ of the final project budget, an alternative solution was sought.

A number of unofficial hardware simulations of the GNS430W and GNS530W are available commercially with varying degrees of fidelity; however, the majority of them were found to be intended exclusively for use with either FSX or Prepar3D, not XPlane. The flight simulation hobbyist community has also produced several attempts at simulated hardware for the GNS430W or GNS530W, the most well-developed of which is the GarSIM GNS530 created by Steve Masters. Masters now offers the GarSIM GNS530 for sale via his company, Emuteq Emulation Technology [109]. The GarSIM GNS530 retails for 445 GPB and consists of a custom faceplate, appropriate buttons and knobs, and a $640 \times 480$ pixel LCD display mounted in a metal enclosure. The display functions as a secondary monitor, while the buttons behave as a secondary USB joystick. The GarSIM GNS530 is shown in Figure 5.40a. 


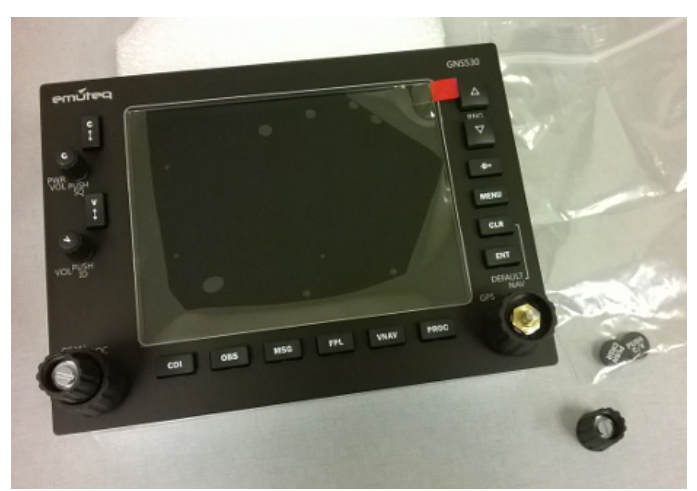

(a) Emuteq GarSIM GNS530. Some minor assembly is required.

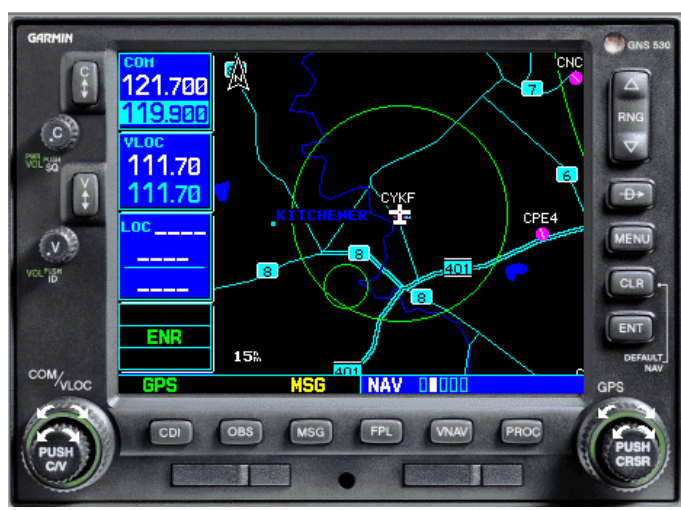

(b) Garmin GNS 530W. (Screenshot from official trainer software [110]).

Figure 5.40: The GarSIM GNS530 by Emuteq (a) is a hardware simulation of the Garmin GNS 530W GPS/NAV/COM device (b).

Masters has released plans for the GarSIM GNS530 to the simulation community $[111,112]$. However, due to time constraints, it was decided to purchase a preassembled unit from Emuteq rather than attempt to create a custom unit based on his work. Use of the commercial GarSIM GNS530 was both cost-effective and compatible with the COTS design philosophy. While WWFC uses the Garmin GNS 430 in their aircraft fleet, use of a simulated GNS 530W in CURVS was approved based on the fact that the two units are very similar in operation. The "GNS 530W XP" GPS plugin for XPlane by RealityXP was selected for use with the GarSIM GNS530 hardware in CURVS [113]. The RealityXP plugin is powered by the official Garmin GNS 530W trainer software, which must also be installed along with the plugin [110].

The GarSIM GNS530 hardware is officially compatible with X-Plane, but only when used with the third-party SimAvio software by FlyThisSim [114]. Unfortunately, at the time of this writing, the SimAvio GNS430W and GNS530W software only included a navigational database for the United States [115]. A worldwide navigational database was planned; however, it had not yet been implemented [116]. As the future home of CURVS is at the Region of Waterloo International Airport $(\mathrm{CYKF})$ in Ontario, Canada, this ready-made solution was not acceptable. 
The lack of X-Plane compatibility is due to the fact that the graphical user interface (GUI) for plugins such as a simulated GPS within X-Plane cannot be "undocked" and moved onto secondary monitors. This is in contrast to FSX and Prepar3D, where undocking of plugins is commonplace. This issue was overcome by Karim Youssef by running a dedicated copy of X-Plane on an auxiliary PC connected directly to the LCD display of the GarSIM GNS530. The commercially-available SmartCopilot plugin was then used to synchronize the GPS PC with the master PC, and send GPS button presses to it [117]. This ensured that operations made on the GPS in the simulator cockpit were mirrored on the IOS display for monitoring by a flight instructor. Two licences for the SmartCopilot plugin were required, at a combined cost of 37.95 USD. Despite requiring an additional PC and X-Plane licence, this method was still significantly less expensive than the purchase of a simulator version of the Garmin 430 which had been previously contemplated.

\subsection{Digital Input}

Just like an actual Cessna 172, the VectorSim included a number of switches for controlling aircraft electrical systems as shown in Figure 5.41a. These switches were originally interfaced to LED indicators on KATIF-1 which would illuminate to show switch positions for troubleshooting purposes as shown in Figure 5.41b. The switch signals on KATIF-1 were then connected to a custom digital input / digital output (DIDO) card in the transputer I/O rack. As this card was obsolete and undocumented, a modern interface solution was required, and a LabJack T7 DAQ was selected for this purpose. 


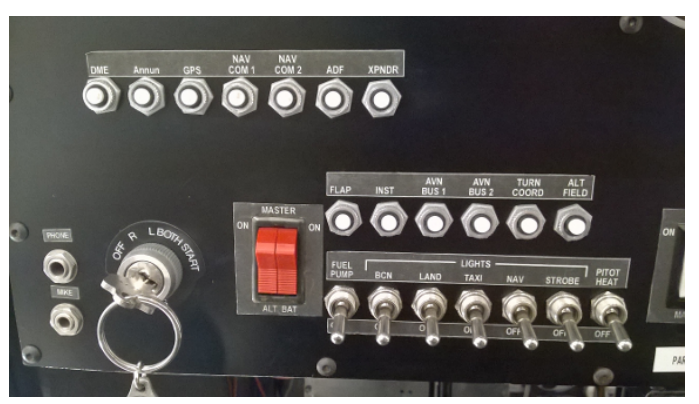

(a) Cockpit key and toggle switches. (White buttons are circuit breakers.)

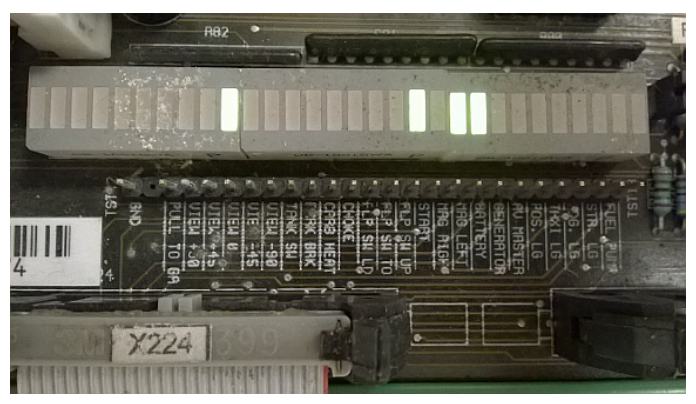

(b) LED switch status indicators.

Figure 5.41: VectorSim switches and associated LED indicators.

Development of a LabJack program to acquire digital inputs from CURVS and pass them to X-Plane was undertaken voluntarily by Neil McFadyen, P.Eng and Supervisor of Computer Operations in the Department of Mechanical and Aerospace Engineering at Carleton University. The general structure of the digital input interface program is shown in Figure 5.42 .

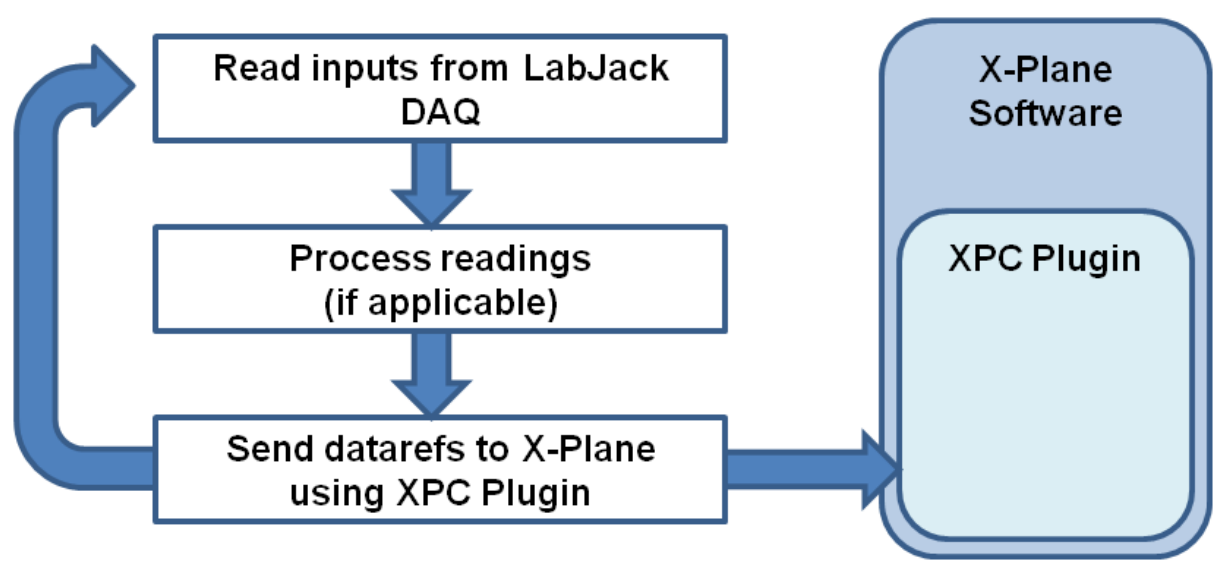

Figure 5.42: CURVS digital input program flowchart.

A complete list of the X-Plane switch datarefs used in CURVS is shown in Table 5.11. In total, there were 19 switch positions in the cockpit to be mapped to these datarefs. An explanation of the function of each switch position is given in Table 5.13. As switches are by their very nature binary, most of the X-Plane switch datarefs are 
also binary and can be toggled between $0(\mathrm{OFF})$ and $1(\mathrm{ON})$ to represent physical switch states. For multi-position switches such as the key and fuel selector switches, a single dataref is used in conjunction with a set of predefined enumerators (e.g., 1, 2, $3,4)$ to represent specific switch positions. While the X-Plane parking_brake_ratio and alternate_static_air_ratio datarefs can accept any value between 0.0 and 1.0, these controls in a Cessna 172 are always either fully OFF (0.0) or fully ON (1.0).

The original ignition switch in the VectorSim was an actual aircraft part and was retained for use in CURVS. This switch required special attention, however, as it had five physical positions, but only three output terminals which could be read by the LabJack. As shown in Table 5.15, the specific position of the switch is represented by the unique combination of output terminals which are HIGH $(\mathrm{H})$ and LOW $(\mathrm{L})$.

Due to to a lack of accurate documentation, locating all of the switch connections on the KATIF-1 output connectors proved difficult. Due in part to time constraints, it was decided to cease reverse-engineering, bypass KATIF-1 entirely, and connect the switches directly to the LabJack digital inputs. The LabJack T7 includes internal $100 \mathrm{k} \Omega$ pull-up resistors on each digital input, making connection of switches simple [119]. External power is not required. A generic example of an external pull-up resistor circuit is shown in Figure 5.43. When the switch is closed, the digital input pin is connected to ground, and therefore measures a LOW voltage. The pullup resistor prevents the establishment of a direct short between the power supply and ground. When the switch is open, the digital input pin is instead exposed to a HIGH voltage. Use of a pullup resistor in combination with a switch ensures that the digital terminal is either HIGH or LOW, and not "floating" at an indeterminate intermediate value [120]. 
Table 5.11: X-Plane switch datarefs used in CURVS. Dataref information adapted from [118].

\begin{tabular}{|l|l|l|}
\hline Switch Name & Dataref Value & Dataref Name \\
\hline Key - Off & 0 & sim/cockpit2/engine/actuators/ignition_key \\
Key - Right & 1 & \\
Key - Left & 2 & \\
Key - Both & 3 & \\
Key - START & 4 & sim/cockpit2/electrical/generator_on \\
\hline Master - ALT & 1,0 & sim/cockpit2/electrical/battery_on \\
\hline Master - BATT & 1,0 & sim/cockpit2/engine/actuators/fuel_pump_on \\
\hline Fuel Pump & 1,0 & sim/cockpit2/switches/beacon_on \\
\hline Beacon & 1,0 & sim/cockpit2/switches/landing_lights_on \\
\hline Landing Light & 1,0 & sim/cockpit2/switches/taxi_light_on \\
\hline Taxi Light & 1,0 & sim/cockpit2/switches/navigation_lights_on \\
\hline Nav Lights & 1,0 & sim/cockpit2/switches/strobe_lights_on \\
\hline Strobe Lights & 1,0 & sim/cockpit2/ice/ice_pitot_heat_on_pilot \\
\hline Pitot Heat & 1,0 & sim/cockpit2/switches/avionics_power_on \\
\hline Avionics Master & 1,0 & sim/cockpit2/controls/parking_brake_ratio \\
\hline Parking Brake & {$[0.0,1.0]$} & sim/cockpit2/switches/alternate_static_air_ratio \\
\hline Alt. Static Air & {$[0.0,1.0]$} & sim/cockpit2/fuel/fuel_tank_selector \\
\hline Fuel - Shutoff & 0 & 1 \\
Fuel - Left & 3 & \\
Fuel - Right & 4 & \\
Fuel - Both & 1,0 & \\
\hline
\end{tabular}


Table 5.13: CURVS switch positions explained.

\begin{tabular}{|l|l|l|}
\hline Number & Switch Positions & Description \\
\hline 1 & Mag R & Right magneto \\
\hline 2 & Mag L & Left magneto \\
\hline 3 & Start & Starter motor \\
\hline 4 & Master - Alternator & Master switch - enables the generator \\
\hline 5 & Master - Battery & Master switch - enables the battery \\
\hline 6 & Fuel Pump & Electric fuel pump \\
\hline 7 & Beacon & Red flashing light on tail \\
\hline 8 & Landing Light & White headlight \\
\hline 9 & Taxi Light & White headlight (reduced brightness) \\
\hline 10 & Navigation Lights & $\begin{array}{l}\text { Red, green, and white lights (left, right, } \\
\text { and tail, respectively) }\end{array}$ \\
\hline 11 & Strobe Lights & Strobes for night visibility \\
\hline 12 & Pitot Heat & $\begin{array}{l}\text { Electric heater to prevent icing of pitot } \\
\text { tube }\end{array}$ \\
\hline 13 & Avionics Master & $\begin{array}{l}\text { Master switch for all electronics (e.g., ra- } \\
\text { dios, GPS) }\end{array}$ \\
\hline 14 & Parking Brake & $\begin{array}{l}\text { Black handle beneath the instrument } \\
\text { panel }\end{array}$ \\
\hline 15 & Alternate Static Air Source & Backup pressure-sampling port \\
\hline 16 & Fuel Shutoff & Master fuel cutoff valve \\
\hline 17 & Fuel Selector - Left & Draw fuel from left tank only \\
\hline 18 & Fuel Selector - Both & Draw fuel from both tanks \\
\hline 19 & Fuel Selector - Right & Draw fuel from right tank only \\
\hline & & \\
\hline 19 & & \\
\hline 19 & &
\end{tabular}


Table 5.15: Ignition switch truth table.

\begin{tabular}{|l|l|l|l|}
\hline & \multicolumn{3}{|c|}{ Output Terminals on KATIF-1 } \\
\hline Physical Switch Positions & Mag L & Mag R & Start \\
\hline OFF & H & H & L \\
\hline Mag R & H & L & L \\
\hline Mag L & L & H & H \\
\hline Both & L & L & L \\
\hline Start & L & L & H \\
\hline
\end{tabular}

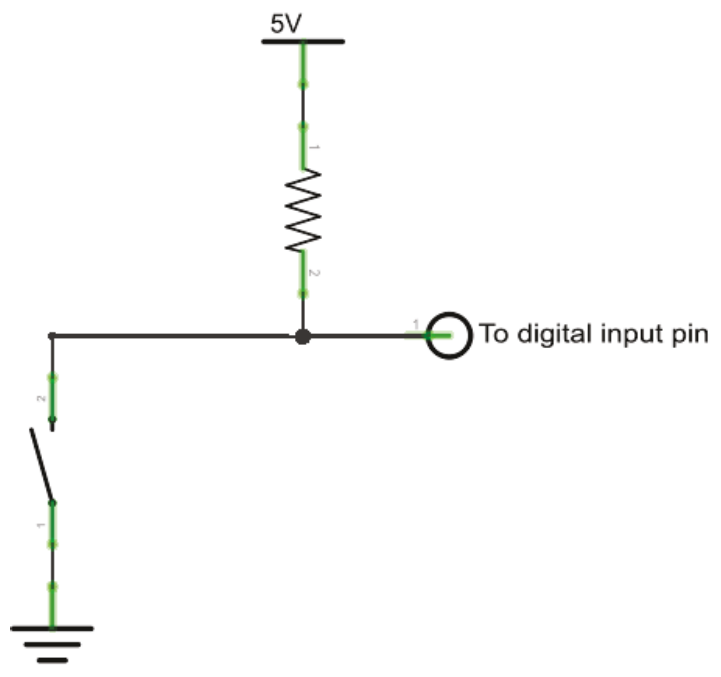

\section{fritzing}

Figure 5.43: A generic pullup resistor circuit. Closing the switch connects the digital input pin to ground, causing it to read LOW. The resistor prevent a direct short to ground when the switch is closed. The digital pin reads HIGH when the switch is open. Schematic created with Fritzing. 


\subsection{Radio Stack}

The original manufacturer of the VectorSim radio stack, Luftfahrtstudio Jungwirth, went bankrupt in 2010. Attempts to contact former employees of the company were unsuccessful. Limited wiring diagrams, courtesy of Roloff and Wilhelmi, were available; however, interface control documents for the communication protocol used by the simulated radio modules were not. The radio modules are shown in Figure 5.44.

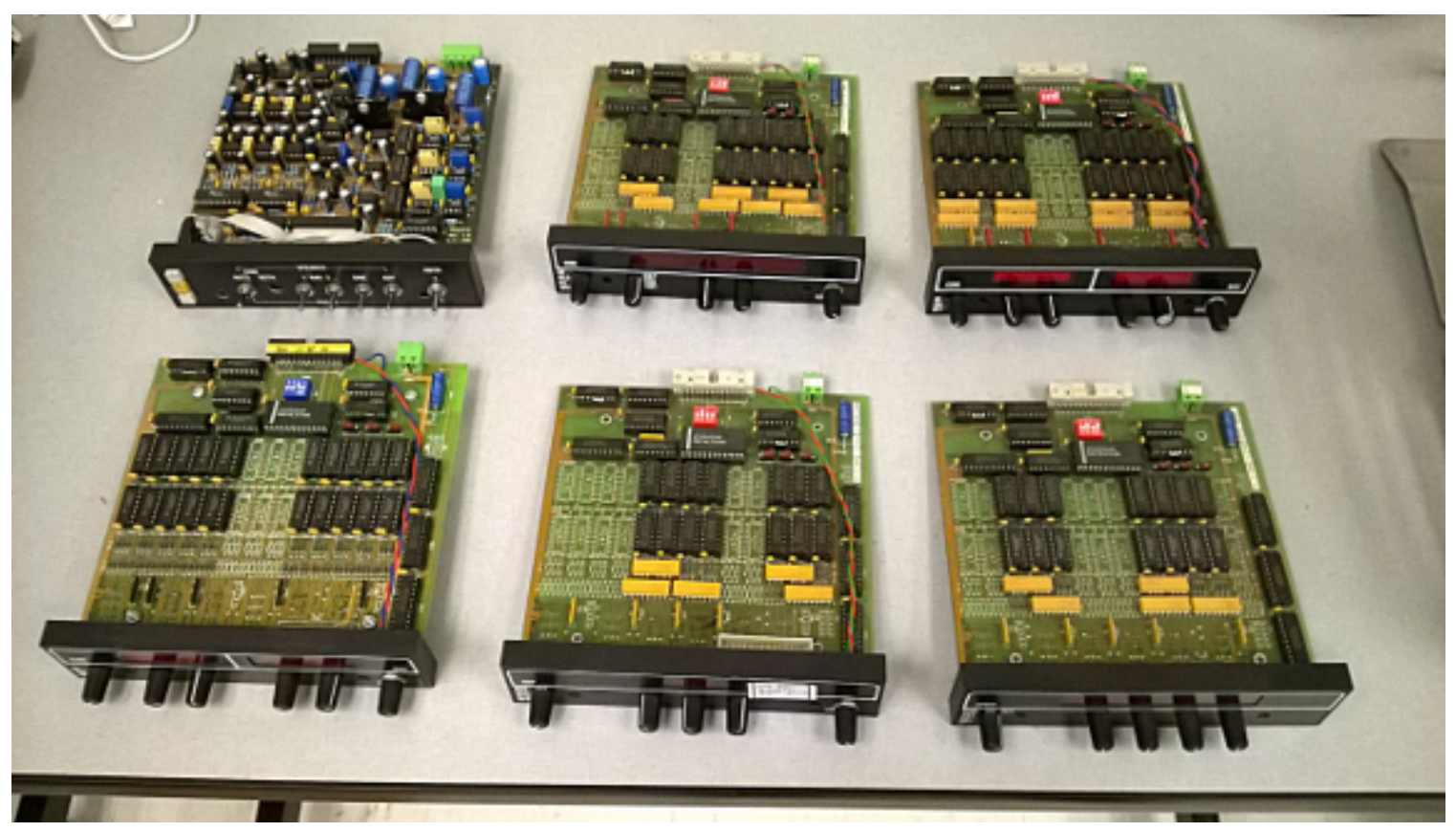

Figure 5.44: Original VectorSim Radio Modules.

Although the simulated radio stack appeared to be still functional under the original transputer system, reverse-engineering of highly-complex, undocumented hardware devices was beyond the scope of the project, and a replacement solution was sought. Working under the oversight of the author, an evaluation of commerciallyavailable simulated radio modules was conducted by Bradley Jung, a fellow graduate student at the ADL. Jung's survey recommended that simulated radio modules from Flight Illusion be used to replace the existing radio stack. Flight Illusion is a manufacturer of simulated avionics based in the Netherlands [121]. 
Since a Garmin 530 acts as a NAV/COM radio in addition to its functions as a GPS receiver, it was decided that only a single simulated Flight Illusion NAV/COM radio would be installed in CURVS. Similarly, as the presence of a GPS eliminates the need for distance measuring equipment (DME), the original simulated DME receiver was removed from the VectorSim and not replaced.

\subsection{Course Deviation Indicator}

A course deviation indicator (CDI) works in conjunction with either the NAV portion of a NAV/COM radio or a GPS. The original Bendix-King CDI in the VectorSim could be toggled between these two modes by means of a button on the GPS annunciation control unit mounted in the instrument panel. For modern GPS units such as the Garmin 530W, this toggle functionality is provided by the "CDI" button on the GPS unit itself. Due to use of an actual Bendix-King KLN-89B GPS receiver in the original simulator, an actual Bendix-King KI 209A CDI was also originally installed, shown in Figure 5.45.

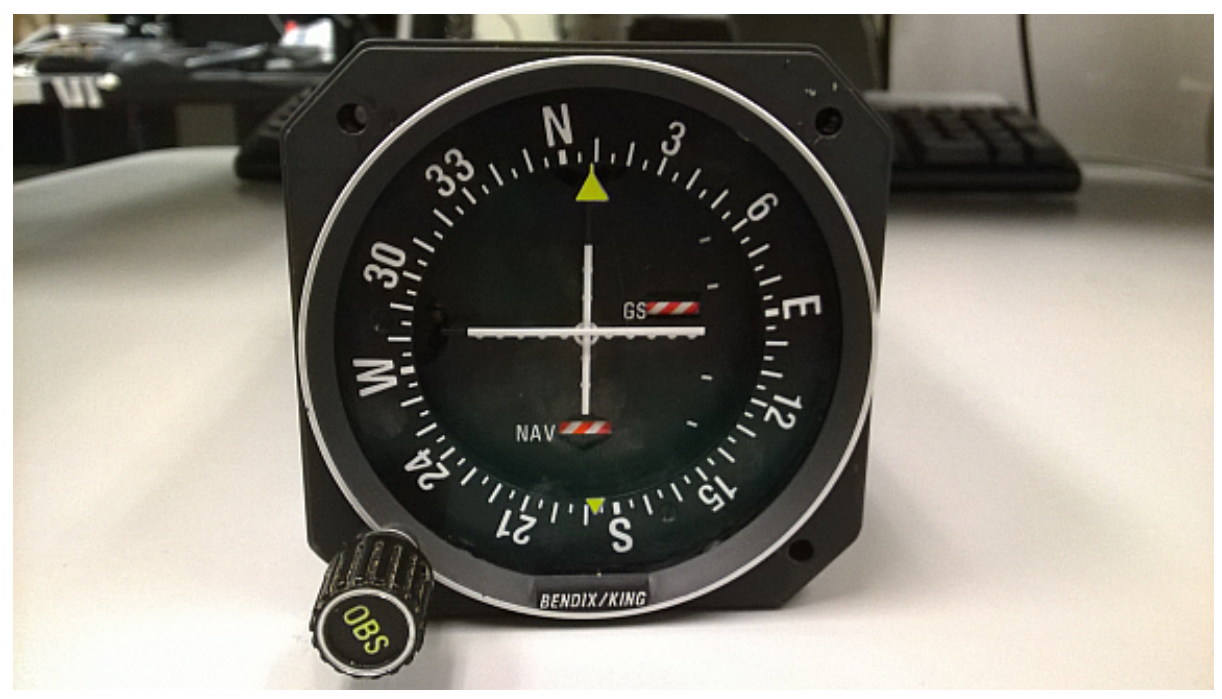

Figure 5.45: The Bendix-King KI 209A CDI, removed from the simulator for inspection. 
A CDI is typically used when flying under instrument flight rules (IFR). Depending on the selected mode, a CDI indicates deviation from a course defined either by radio beacons or a GPS. In the context of a CDI, radio beacons consist of very high frequency omnidirectional range (VOR) stations for cross-country navigation, along with localizer (LOC) and glideslope (GS) transmitters for runways equipped with an instrument landing system (ILS).

Preliminary reverse-engineering work on the original CDI was conducted jointly by the author and Karim Youssef. Reference was made both to the device itself, shown disassembled in Figure 5.46, and the available Bendix-King documentation [122]. It was found that a system of internal relays within the CDI made it possible to bypass the internal VOR/LOC converter, and drive the instrument using external analogue signals, such as those provided by a GPS [123]. In addition, the resolver connected to the omni-bearing selector (OBS) knob on the front of the unit was found to have very unique power requirements (30 Hz AC) [124]. However, it was ultimately decided that reverse-engineering a completely unique instrument was not possible within the time constraints of the project. Thus, a simulated CDI from Flight Illusion was selected as a replacement [125]. This solution also had the great advantage of being natively X-Plane compatible. 


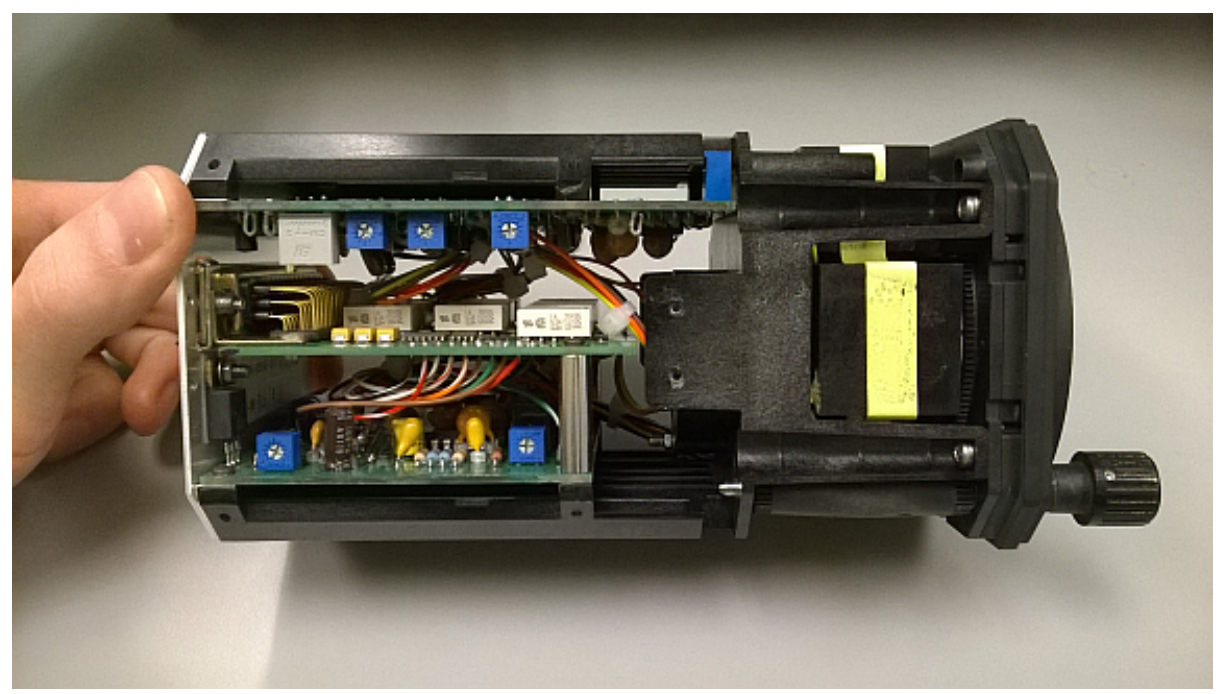

Figure 5.46: The Bendix-King KI 209A CDI with casing removed.

\subsection{Circuit Breakers}

WWFC conducts training in the emergency procedures to be used in the event of an electrical system failure as part of both the private pilot licence (PPL) and the night rating. Thus, restoration of the circuit breaker subsystem was important to the customer. Regarding circuit breakers which trip in flight, the Transport Canada Aeronautical Information Manual recommends that tripped breakers never be reset, except possibly in the case of essential equipment, and then only once [126]. The purpose of this recommendation is to eliminate the possibility of starting an electrical fire in flight due to the continuous re-enabling of faulty equipment.

The circuit breakers originally installed in the VectorSim were found to be actual, as opposed to simulated units. The technical specifications for the VectorSim circuit breakers are shown in Table 5.17.

The circuit breaker control circuitry was located on the second so-called Katana interface card (KATIF-2), positioned on the right-hand side of the simulator from the pilot's perspective. The portion of KATIF-2 which is responsible for tripping and sensing the state of the circuit breakers is shown in Figure 5.47. 
Table 5.17: VectorSim circuit breaker specifications.

\begin{tabular}{|l|l|}
\hline \multicolumn{2}{|c|}{ Electric Trim Circuit Breaker } \\
\hline Manufacturer & Potter \& Brumfield \\
\hline Model & W23-X1A1G-0.50 \\
\hline Specifications & $0.5 \mathrm{~A}, 250 \mathrm{VAC} / 50 \mathrm{VDC}$ \\
\hline Datasheet & {$[127]$} \\
\hline \multicolumn{2}{|c|}{ All Other Circuit Breakers } \\
\hline Manufacturer & Mechanical Products Co. \\
\hline Model & $1681-002-050$ (Series 16) \\
\hline Specifications & $0.5 \mathrm{~A}, 250 \mathrm{VAC} / 50 \mathrm{VDC}$ \\
\hline Datasheet & {$[128]$} \\
\hline
\end{tabular}

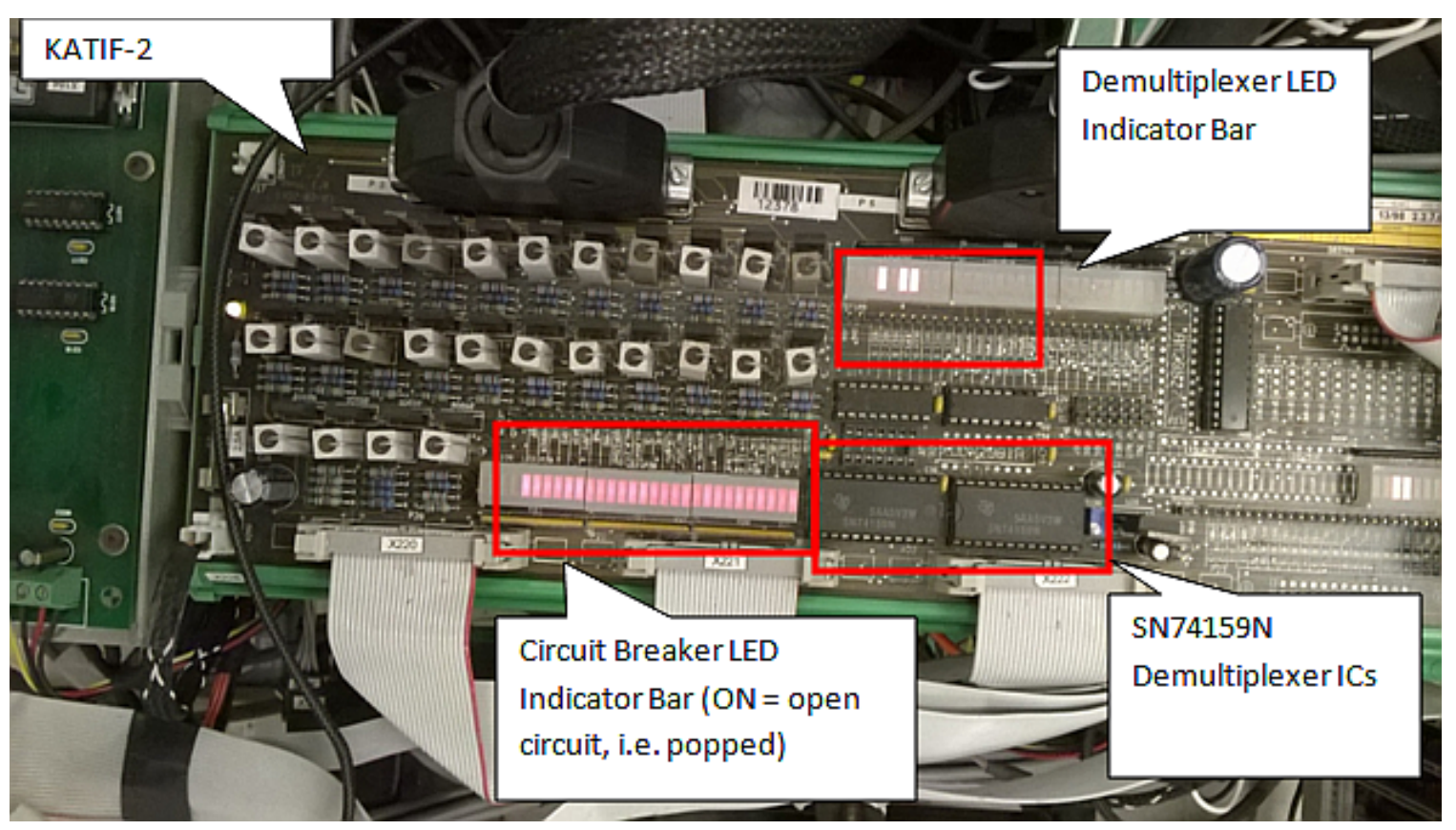

Figure 5.47: Circuit breaker trip and sense circuitry on KATIF-2. 
KATIF-2 contained circuitry to support up to 26 circuit breakers, as this is what a Diamond Katana aircraft requires. However, as a Cessna 172 only uses 14 circuit breakers, the remaining 12 circuit breaker channels were not used. The state of each circuit breaker was shown by a bar of red LED indicators.

\subsubsection{Reverse-engineering the Circuit Breaker Subsystem}

While most of the circuit breakers could not be toggled by hand due to their design, the electric trim circuit breaker was designed to be able to be manually toggled if desired. By toggling this breaker, it was found that the "TRIM" LED indicator on KATIF-2 illuminated only when the breaker was in the tripped, or open-circuit, state. Since 12 of the LEDs in the light bar remained illuminated at all times, even when all 14 cockpit breakers were pushed in (i.e., closed-circuit state), it was concluded that these 12 LEDs represented breakers which were not installed, and thus always registered as open circuits.

Unfortunately, the labels printed on the KATIF-2 circuit board next to the LED indicators did not necessarily match the labels on the breakers in the cockpit. This was due to the fact that the KATIF-2 board was originally designed to simulate a Diamond Katana, not a Cessna 172. In order to determine which breakers were connected to which LED indicators, the original VectorSim test program was run on the original IOS PC. This test program was used to access the embedded transputer and remotely trip all the breakers in the cockpit. Tripping the breakers caused all 26 LEDs in the circuit breaker light bar to illuminate, as shown in Figure 5.48.

The cockpit breakers were then pushed in one-by-one. This caused the corresponding LED indicators to turn off, allowing each circuit breaker to be positively matched to the appropriate indicator. The mapping between each cockpit circuit breaker (CB) and the corresponding KATIF-2 LED indicator label is shown in Table 5.18.

As is also shown in Figure 5.48, a row of test pins was situated on KATIF-2 next 
Table 5.18: VectorSim circuit breaker mappings. Note that numbering is not sequential due to unused circuit breaker channels.

\begin{tabular}{|l|l|l|}
\hline CB $\#$ & Label on CB in Cockpit & Label on LED Indicator on KATIF-2 \\
\hline 1 & ALT. FIELD & CHT/OIL \\
\hline 3 & GPS & FUEL P. \\
\hline 9 & INST. & START \\
\hline 13 & NAV/COM 1 & COM.NAV \\
\hline 14 & XPNDR & ATC \\
\hline 16 & AVN. BUS 1 & AV. MAST. \\
\hline 17 & AVN. BUS 2 & AV. M. CT. \\
\hline 20 & TURN COORD. & T\&B \\
\hline 21 & FLAPS & FLAPS \\
\hline 22 & E-TRIM & TRIM \\
\hline 23 & NAV/COM 2 & COM2 \\
\hline 24 & DME & DME \\
\hline 25 & ANNUN. & MARKER \\
\hline 26 & ADF & ADF \\
\hline
\end{tabular}




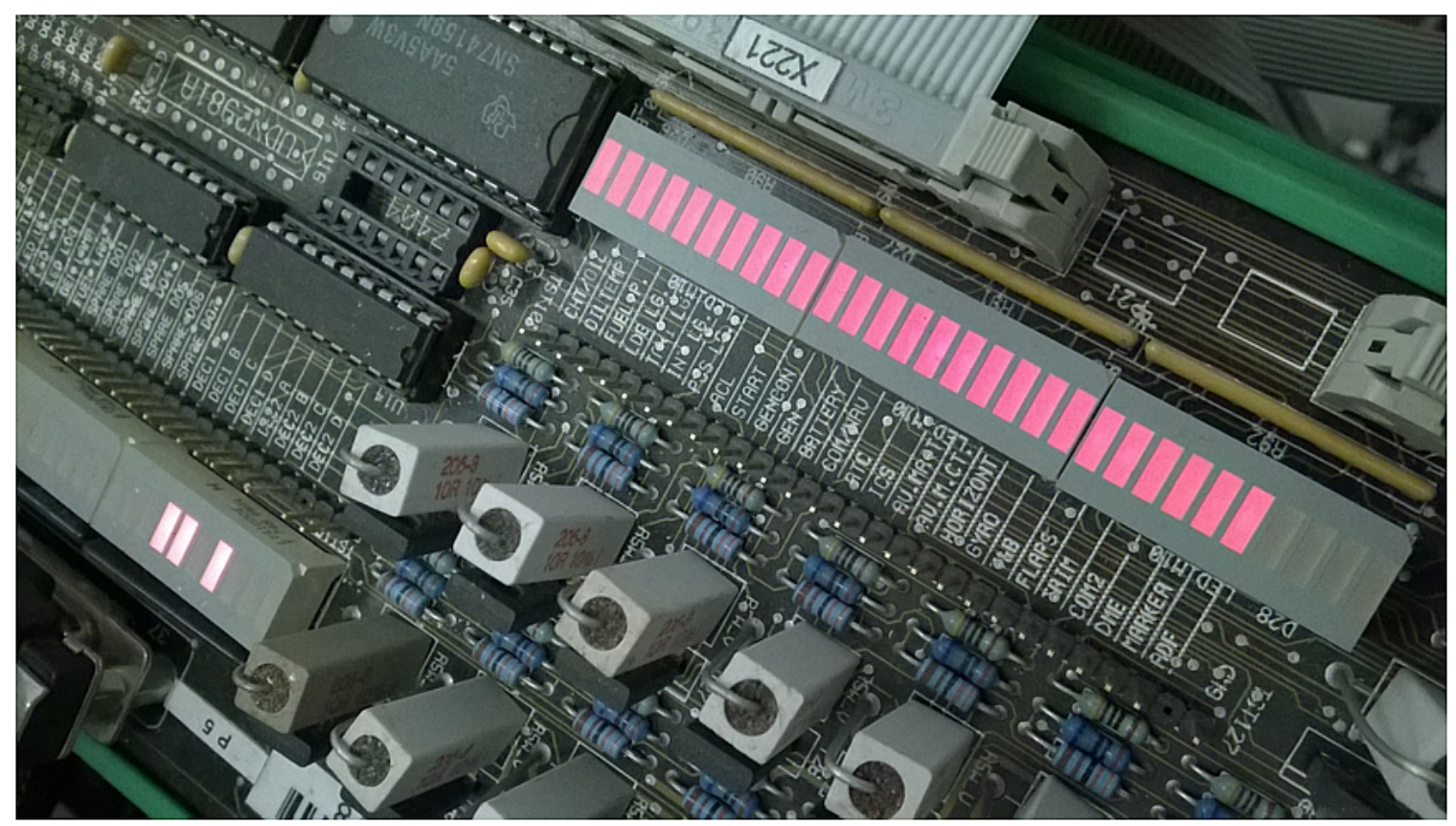

Figure 5.48: Circuit breaker LED indicator bar on KATIF-2. Illuminated LEDs indicate an open-circuit (tripped) state. Note the line of test pins next to the labels.

to the LED indicator strip. The voltages on each of these pins was measured for each circuit breaker state (tripped/un-tripped). The results of these tests are shown in Table 5.19.

Table 5.19: VectorSim circuit breaker test pin voltages for the tripped and untripped states.

\begin{tabular}{|l|l|l|}
\hline & CB Tripped & CB Un-Tripped \\
\hline Voltage at Test Pins & $\leq 0.85 \mathrm{~V}$ & $\geq 3.27 \mathrm{~V}$ \\
\hline
\end{tabular}

Thus, for data acquisition purposes, and allowing a margin for measurement error, it was concluded that if the voltage at a given circuit breaker test pin is:

- $\geq 3 \mathrm{~V} \rightarrow$ circuit breaker is un-tripped (closed circuit)

- $\leq 0.9 \mathrm{~V} \rightarrow$ circuit breaker is tripped (open circuit)

Referring to the datasheet for the LabJack T7 DAQ, it was found that these ranges 
were not quite compatible with the logic level transition values used by the digital inputs on the device [129]. An extract from the relevant section of the datasheet is shown in Table 5.20.

Table 5.20: Logic level transition values for the digital inputs on the LabJack T7 DAQ. Reproduced from [129].

\begin{tabular}{|l|l|l|l|}
\hline Parameter & Min & Max & Units \\
\hline Low Level Input Voltage & -0.3 & 0.5 & Volts \\
\hline High Level Input Voltage & 2.64 & 5.8 & Volts \\
\hline
\end{tabular}

However, as the LabJack T7 also features 14 analogue inputs which are $\pm 10 \mathrm{~V}$ tolerant, it was instead decided to implement custom logic transition levels in software in order to detect the state of each circuit breaker. While it would have been relatively simple to implement voltage dividers to decrease the tripped voltages below the logiclow level of the LabJack T7, it was simpler to just use the available analogue inputs instead. This decision was also in keeping with the project philosophy of using COTS parts wherever possible in the interest of simplicity and maintainability.

\subsubsection{The Process of Tripping Circuit Breakers}

From the original schematics provided by Jared Wilhelmi, it was seen that two Texas Instruments SN74159N integrated circuits were used in the control circuitry for the breakers. However, due to reproduction degradation, it was not initially clear how they were used. According to the datasheet, the SN74159N is a 4-line to 16-line decoder/demultiplexer with open-collector outputs [130]. This chip decodes a 4-bit input signal (on input channels A, B, C, and D) into a single output, causing the appropriate output channel to go LOW (i.e., be connected to ground).

Thus, this chip allowed 4 data lines to control a total of 16 output lines. It was hypothesized that this functionality was used by KATIF-2 to control the breakers 
in the cockpit, while also minimizing the number of control lines required. This hypothesis was tested by observing the complete set of LED indicators on KATIF-2 while tripping the cockpit breakers using the original IOS station. As each breaker was selected for tripping, it was observed that a unique sequence of 1-3 LEDs would illuminate on a particular LED indicator bar. Closer examination of this LED bar revealed notations referring to DEC 1 and DEC 2, each consisting of channels $\mathrm{A}, \mathrm{B}$, C, and D. Thus, it was seen that as each breaker was triggered, a 4-bit code was being input to one or the other of the decoder chips. This in turn caused an over-current to be applied to the appropriate breaker, causing it to trip (pop out) after a few seconds.

It was interesting to note that in the few seconds between when an un-tripped breaker was selected and when it actually tripped, the corresponding LED indicator for the breaker was observed to glow dimly. In this semi-lit state, the corresponding test pin voltage was found to be approximately $1 \mathrm{~V}$ below what would be expected for a breaker in its un-tripped state (e.g., $2.28 \mathrm{~V}$ instead of $3.28 \mathrm{~V}$ ). This dim illumination of the LED breaker indicator made it possible to see visually when the simulator was attempting to trip a breaker which was not already tripped.

After tripping, a few seconds were found to be required for a given circuit breaker to allow itself to be reset. This was signalled by a slight metallic clicking sound from within the breaker, only after which could it be reset.

As the original design (i.e., Diamond Katana simulator) required 26 breakers, two decoder chips were used, each with 16 outputs. Since only 14 breakers are required for a Cessna 172, only one decoder chip is strictly required. However, due to the way the VectorSim was constructed, the breakers were found to be split across both chips. One decoder chip controls the first 5 breakers, while the remaining 9 breakers are controlled by the other. As shown in Table 5.21, a truth table for the decoder chips was compiled based on which indicators were shown on the decoder LED indicator bar. 
Table 5.21: VectorSim circuit breaker truth table. In order to trip a given circuit breaker, the corresponding decoder inputs must be set electrically high $(\mathrm{H})$ or low (L) in the specified pattern.

\begin{tabular}{|l|l|l|l|l|l|l|l|l|l|}
\hline & & \multicolumn{9}{|c|}{ Decoder Inputs "DEC 1" } & \multicolumn{3}{|c|}{ Decoder Inputs "DEC 2" } \\
\hline CB \# & Cockpit Label & A & B & C & D & A & B & C & D \\
\hline 1 & ALT. FIELD & H & L & L & L & & & & \\
\hline 3 & GPS & H & H & L & L & & & & \\
\hline 9 & INST. & H & L & L & H & & & & \\
\hline 13 & NAV/COM 1 & H & L & H & H & & & & \\
\hline 14 & XPNDR & L & H & H & H & & & & \\
\hline 16 & AVN. BUS 1 & & & & & H & L & L & L \\
\hline 17 & AVN. BUS 2 & & & & & L & H & L & L \\
\hline 20 & TURN COORD. & & & & & H & L & H & L \\
\hline 21 & FLAPS & & & & & L & H & H & L \\
\hline 22 & E-TRIM & & & & & H & H & H & L \\
\hline 23 & NAV/COM 2 & & & & & L & L & L & H \\
\hline 24 & DME & & & & & H & L & L & H \\
\hline 25 & ANNUN. & & & & & L & H & L & H \\
\hline 26 & ADF & & & & & H & H & L & H \\
\hline
\end{tabular}




\subsubsection{Circuit Breaker Datarefs}

Unfortunately, the default Cessna 172 model included with X-Plane 10 does not simulate the circuit breaker subsystem, and therefore no circuit breaker datarefs are available. Three potential solutions to this problem were considered, as shown below:

1. Trip circuit breakers as a side-effect of other relevant system failures;

2. Trip circuit breakers separately from X-Plane; or

3. Use a thrid-party Cessna 172 model which simulates the circuit breaker subsystem.

The first option would have attempted to associate circuit breaker activation with appropriate, pre-defined system failures already present in X-Plane. This option was discarded due to the length of time it would likely have required to implement.

The second option would have involved the creation of an external, standalone program which would have allowed the instructor to activate circuit breakers on command, in conjunction with relevant X-Plane failures if desired. The option of triggering circuit breakers separately from predefined failures has been used in the past at the ADL on the KatanaSim project [9]. A similar approach is also currently used by the ALSIM ALX simulator currently in use at WWFC. However, this option was also discarded due to the increased workload it would have placed on the instructor to devise and implement failure scenarios.

The third option was to locate and use a pre-existing Cessna $172 \mathrm{~S}$ model with a simulation of the circuit breaker subsystem already implemented. At the time of this writing, only one suitable third-party model was available: the Cessna 172SP by Airfoil Labs (34.95 USD) [131,132]. This model included working circuit breakers and associated datarefs and was actively supported by the developer. Thus, due to its compliance with both the COTS and cost-effective design philosophies, this solution 
was selected for use in CURVS. An interesting feature of the X-Plane LAN architecture is that the aircraft model used on the slave PCs does not have to be identical to that used on the master. This allowed a single copy of the Airfoil Labs model to be run on the master PC, with the default X-Plane Cessna 172 model continuing to be used on each of the slave PCs. This feature was important as the 3D mesh of the default model had been specially-modified by the author's colleague Hooman Rajaee to only include the wings and propeller. Use of actual 3D aircraft components on-screen instead of static overlay images increased visual fidelity as it allowed the propeller to rotate appropriately, control surface deflections to be observed, and X-Plane lighting effects such as sun-glint to be applied to the wings. 


\section{Chapter 6}

\section{Supporting Systems}

In addition to the work described in the Chapter 5, a number of other simulator systems were also redeveloped and modernized. Due to the large scope of the project, several redevelopment tasks were reassigned to Hooman Rajaee, a fellow graduate student at the ADL. These systems were implemented with the assistance and under the oversight of the author. Further details and analysis are available in Rajaee's Masters thesis [133]. In order to give a complete picture of the redevelopment work which was performed, a brief overview appears below.

\subsection{Projection System}

Three high-definition short-throw home theatre projectors were selected to replace the single, low-resolution original projector. The BenQ HT1085ST projector was selected due its cost-effectiveness (approximately 1000 CAD per unit) and overall user satisfaction [134]. These projectors featured digital light processing (DLP) technology, in which millions of microscopic mirrors are used to reflect incident light either towards or away from the lens in order to turn individual pixels "on" or "off," respectively. The HT1085ST projector and an illustration of a single DLP micromirror are shown in Figure 6.1. 


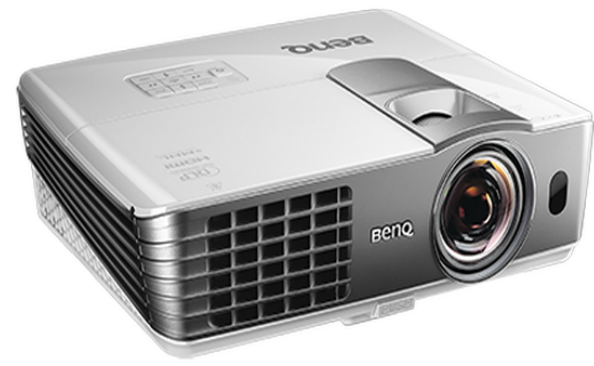

(a) BenQ HT1085ST short-throw highdefinition DLP projector [134].

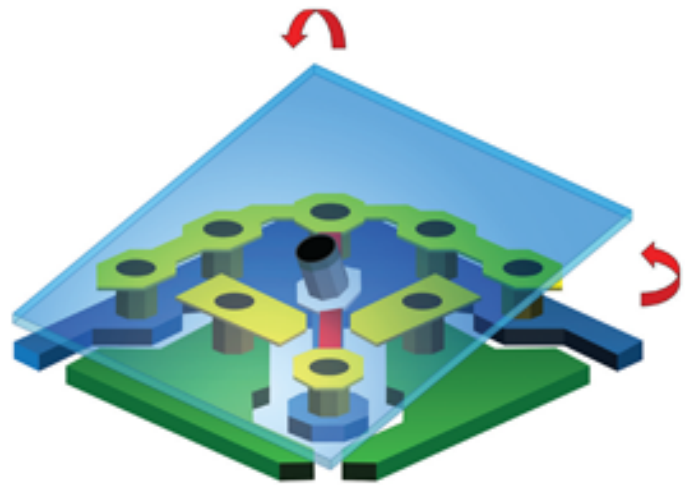

(b) A single DLP micromirror [135]. Each mirror is approximately onefifth the diameter of a human hair.

Figure 6.1

In order to form a full-colour image, single-chip DLP projectors such as the HT1085ST pass white light through a rotating colour filter or "wheel," with red $(\mathrm{R})$, green $(\mathrm{G})$, and blue $(\mathrm{B})$ segments before allowing it to fall onto the DLP chip. The red, green, and blue components of each frame are then projected in sequence to form a full-colour image on screen, as illustrated in Figure 6.2. Some DLP colour wheels also include segments for some non-primary colours [136].
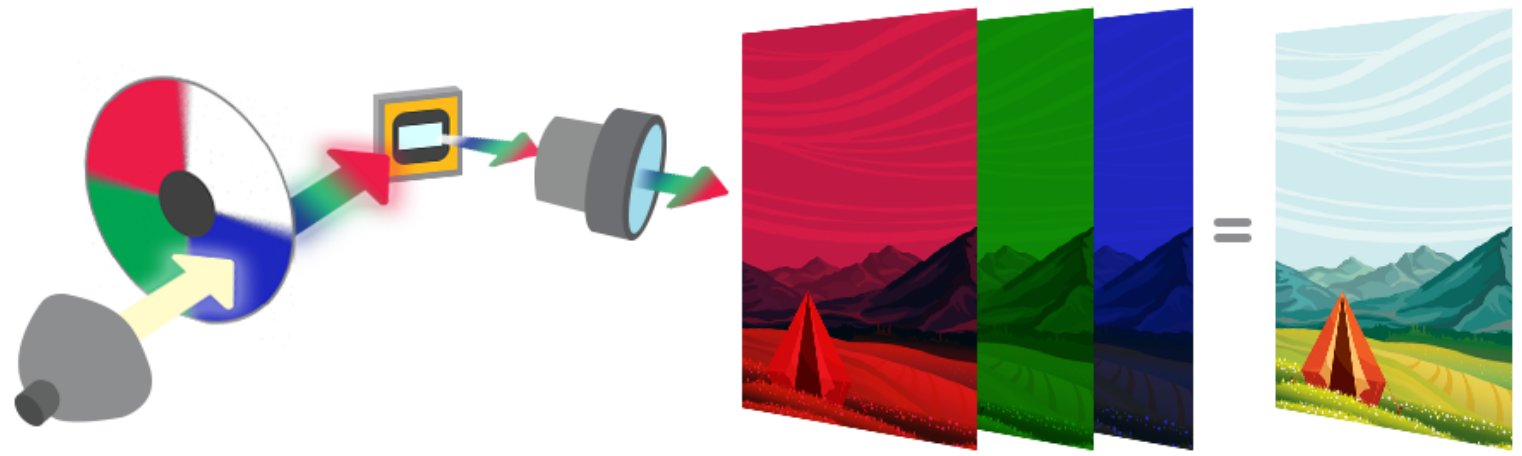

Figure 6.2: The colour wheel in a single-chip DLP projector allows the primary colours of each frame to be rapidly projected in sequence to form a full-colour image [137]. This particular colour wheel also includes a white (W) segment.

This single-chip projection technique can suffer from visual artefacts colloquially referred to as the "rainbow effect," in which traces of the individual primary-colour 
subframes are visible to some viewers. However, in practice, the HT1085ST was found to be immune to the rainbow effect, likely due to its use of a six-segment RGBRGB colour wheel rotating at six times the projection refresh rate of $60 \mathrm{~Hz}$ [138].

\subsubsection{Cylindrical Projection Screen}

Due to the extremely wide horizontal field of view decided upon by the author for the redeveloped projection system and the expense of existing commercially-available options, a custom $220^{\circ}$ screen was designed and fabricated. The frame of the screen was constructed from steel pipe connected together by structural clamps. The screen material was polyvinyl chloride (PVC), and was made to order. The completed projection screen is shown in Figure 6.3. The screen was designed such that it could be easily disassembled for transport to WWFC.

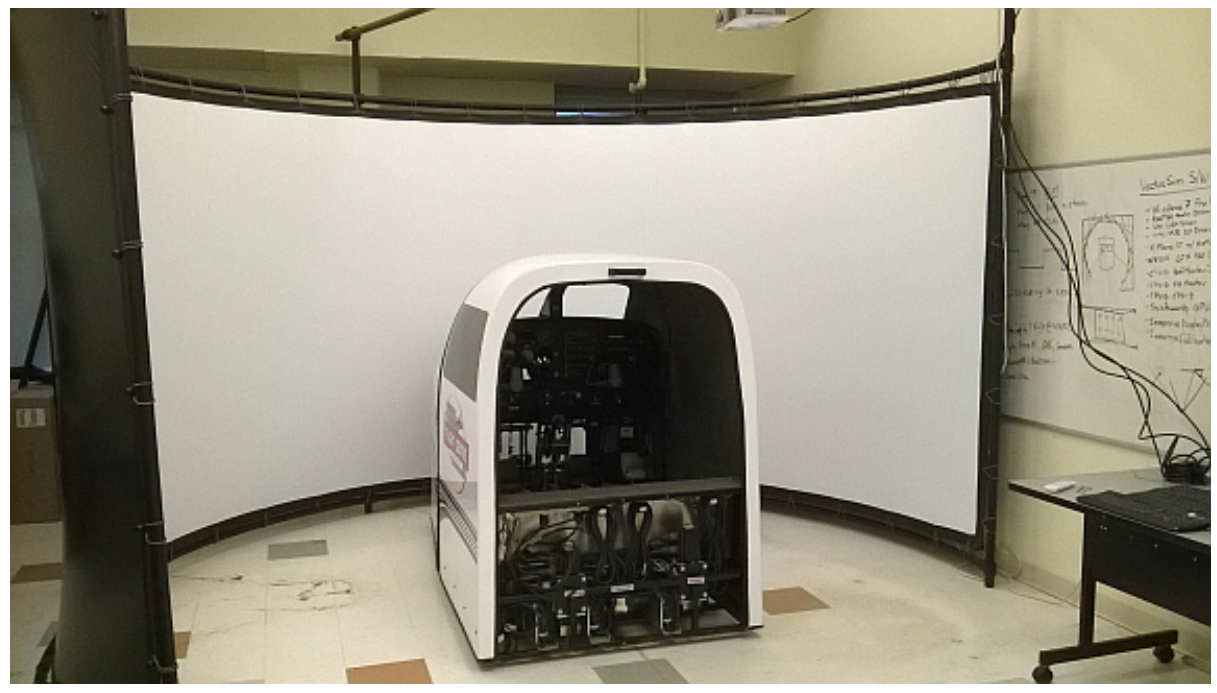

Figure 6.3: The new CURVS projection screen. The projector mounting plate is not shown. 


\subsubsection{Image-warping Software}

In order to use a curved projection screen, the projected image must first be prewarped to account for the non-flat geometry of the screen. This process is known as geometric correction. Furthermore, when using multiple projectors, the edges of each image must be seamlessly blended with the adjacent images. The cost-effective "Immersive Display PRO" and "Immersive Calibration PRO" software packages from Fly Elise-ng were selected for these purposes [139]. The total cost of licences for three image generation PCs was 891 EUR (approximately 1,271 CAD). Although professional-use X-Plane licences were still required at a cost of 750 USD/licence, this approach allowed a savings of approximately 1000 CAD compared with the cost of purchasing three projection-enabled X-Plane licences at 1,500 USD each.

A comparison of unwarped and warped image projection onto a curved screen is shown in Figure 6.4 .

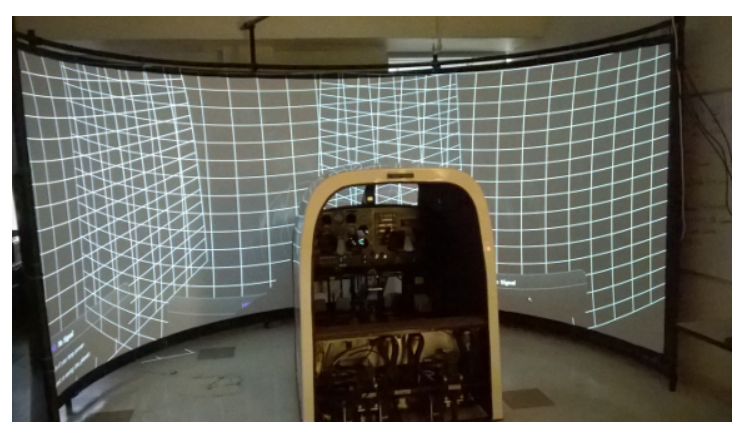

(a) Projecting unwarped grid patterns onto a curved screen produces distortion, with obvious overlap between images.

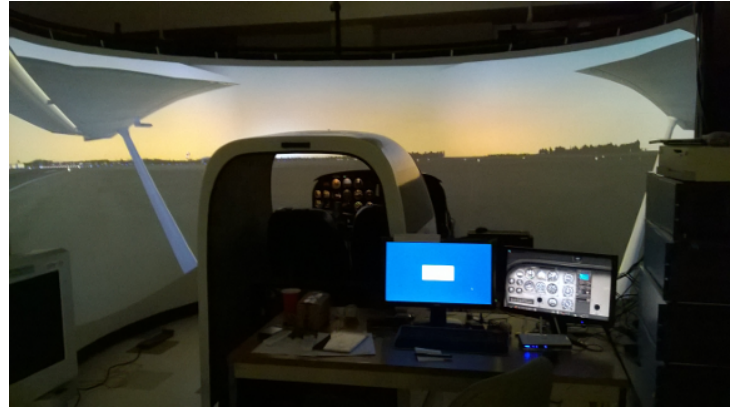

(b) After warping and soft-edge blending, projected images are geometrically correct, and the joins between images invisible.

Figure 6.4 


\subsubsection{Custom Scenery for CYKF}

By default, X-Plane included only generic scenery for Waterloo-Wellington International Airport (CYKF). While it included the two runways, the taxiways were inaccurate, and airport buildings were non-existent. Since WWFC is based at CYKF, high-fidelity scenery was required. A highly-detailed custom scenery package was developed which featured satellite imagery, custom-made buildings, and accurate taxiways, signs, markings, etc. As per the Transport Canada preference, operational windsocks were also included. An example of the custom scenery is shown in Figure 6.5. In order to increase realism off-airport, the third version of the freeware "HD Mesh Scenery" add-on scenery package by Andras Fabian was used with his gracious permission [70].

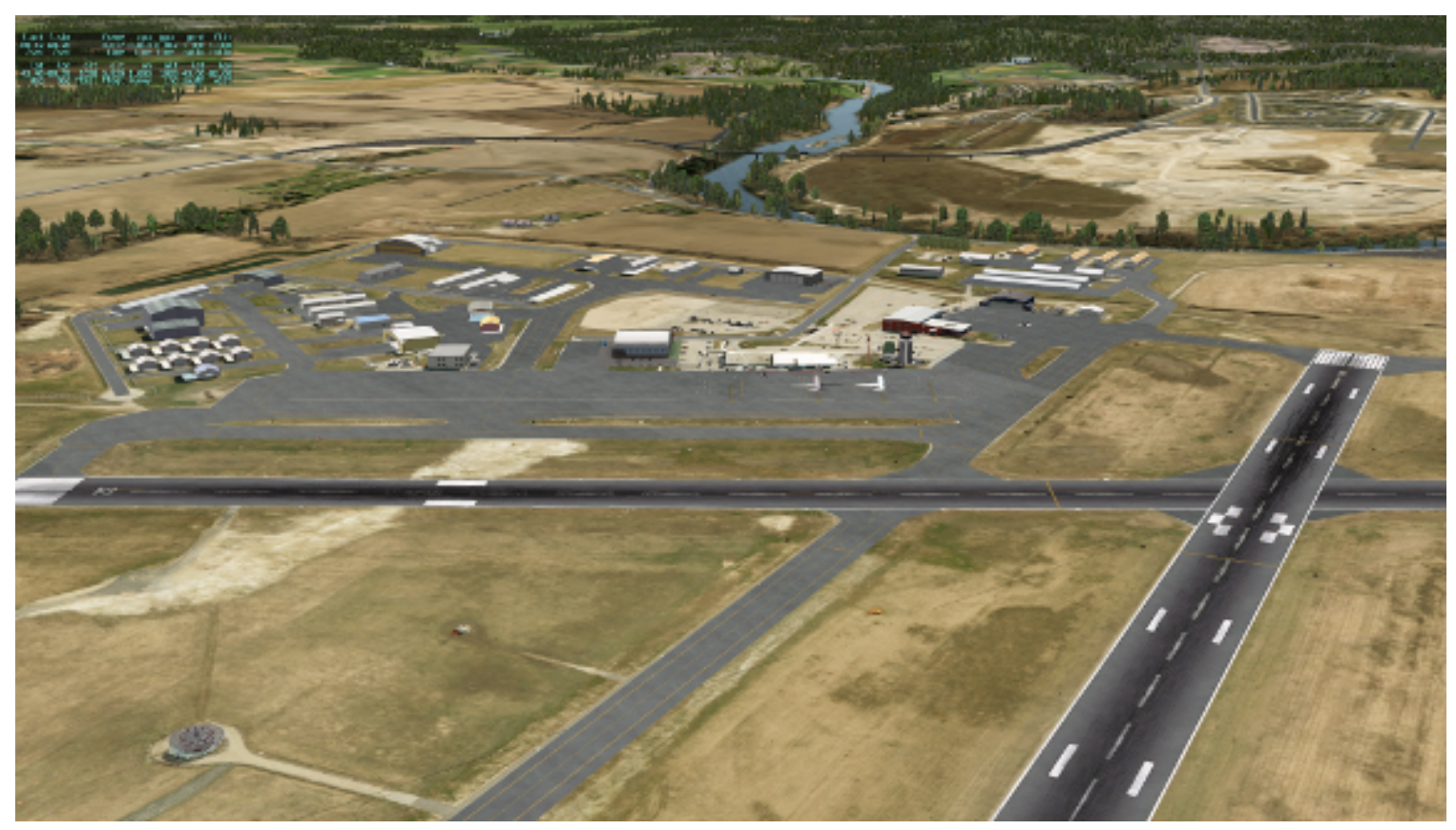

Figure 6.5: Custom scenery for Waterloo Wellington International Airport (CYKF). Image by Hooman Rajaee. 


\subsection{Audio}

In order to increase fidelity, the default X-Plane audio was replaced with custom audio recordings from an actual Cessna 172, shown in Figure 6.6. The custom engine audio featured more prominent bass content than did the X-Plane default, and improved realism appreciably.

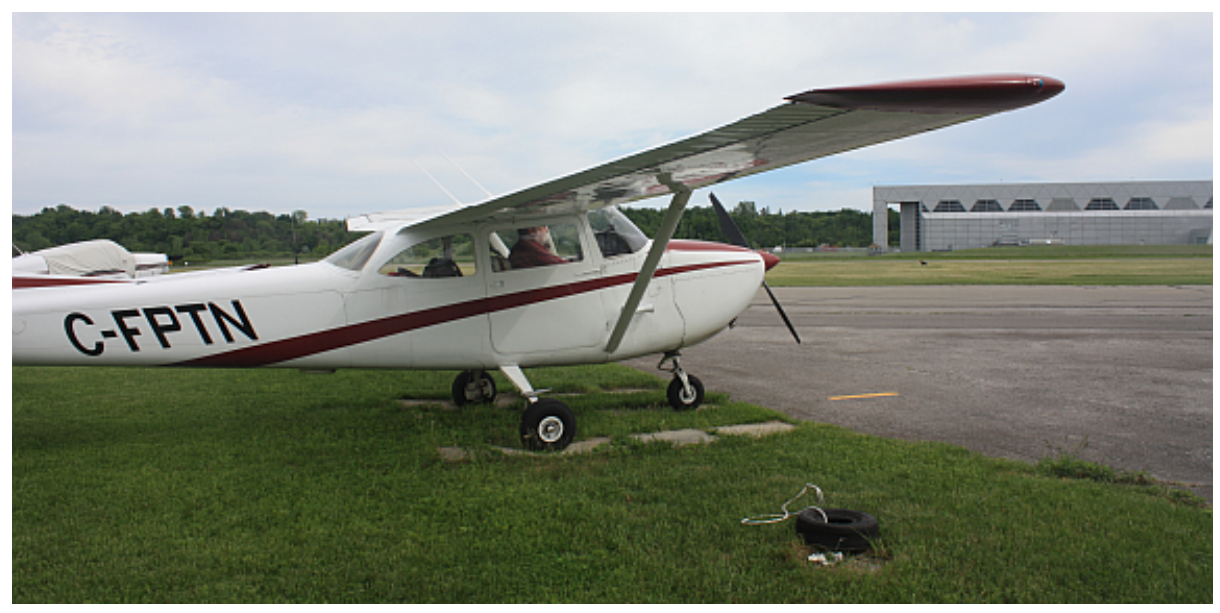

Figure 6.6: Custom high-fidelity audio recordings were created using a Cessna 172E, registration C-FTPN. Image by Hooman Rajaee.

\subsection{Vibration}

In order to increase immersion, two Buttkicker ${ }^{\circledR}$-brand vibration transducers were mounted beneath the seats in the simulator [140]. These seat-shakers provided additional tactile feedback to the pilot regarding engine RPM and ground contact. This latter feature allows pilots in CURVS to directly sense the exact moment of transition from ground to air or vice-versa, as this can be otherwise difficult to detect in a simulation. 


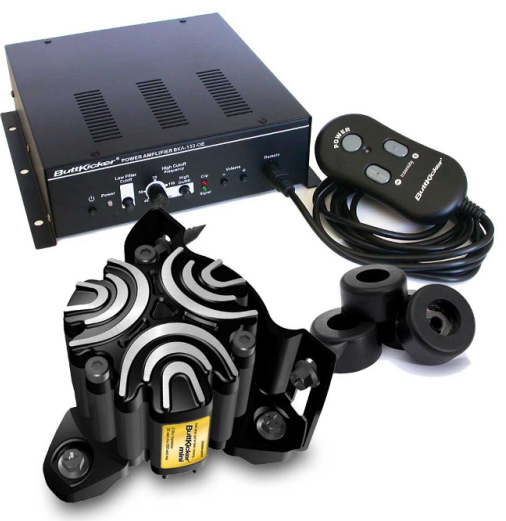

Figure 6.7: The Buttkicker ${ }^{\circledR}$ Simulation kit, consisting of vibration transducer, amplifier, remote control, and vibration isolators. Image by The Guitammer Company. 


\section{Chapter 7}

\section{CURVS: The Big Picture}

The preceding two chapters have dealt with individual subsystem of CURVS. The purpose of this chapter is to describe how the individual hardware and software components worked together as a system in the context of a typical simulation session.

\subsection{CURVS Startup}

A typical simulation session is begun by powering on the individual components which make up CURVS. In order to ensure that all steps were performed in the correct order, a checklist was developed. The checklist followed the typical "challenge-response" format common in the aviation industry and is shown in Table 7.1. 
Table 7.1: CURVS startup checklist.

\begin{tabular}{|l||l|}
\hline Challenge & Response \\
\hline Simulation PCs & ON \\
\hline Projectors & ON (use remote control) \\
\hline Control loading & DISABLE (E-stop button DOWN) \\
\hline Simulator power bar & ON \\
\hline Buttkicker & ON (verify GREEN light) \\
\hline Simulator power & $\begin{array}{l}\text { ON (red rocker switch behind pilots } \\
\text { seat) }\end{array}$ \\
\hline LabJack power bar & ON \\
\hline X-Plane auto-start complete & VERIFY \\
\hline $\begin{array}{l}\text { CURVS Analogue Input \& Control } \\
\text { Loading Interface Program }\end{array}$ & RUN (Use shortcut on Desktop) \\
\hline $\begin{array}{l}\text { CURVS Servo Instrument Interface Pro- } \\
\text { gram }\end{array}$ & RUN (Use shortcut on Desktop) \\
\hline CURVS DIO Interface Program & RUN (Use shortcut on Desktop) \\
\hline $\begin{array}{l}\text { Yoke \& pedals centred (esp. yoke pitch } \\
\text { axis) }\end{array}$ & VERIFY \\
\hline Pilot(s) clear of yoke \& pedals & CHECK \\
\hline Pilot(s) ready for control force & ALERT \\
\hline Control loading & ENABLE (E-stop button UP) \\
\hline
\end{tabular}




\subsection{A Typical Simulation Session}

After power-up of the simulator has been completed, actual flight training may begin. Using the master, or "instructor station" PC, the flight instructor sets up the simulation scenario by selecting the airport, weather, time of day, and starting location from within the X-Plane software.

As most flights are expected to take place starting from Waterloo Wellington International Airport (CYKF), this airport features custom-designed, high-fidelity add-on scenery developed by the author's colleague Hooman Rajaee, examples of which are shown in Figure 7.1.

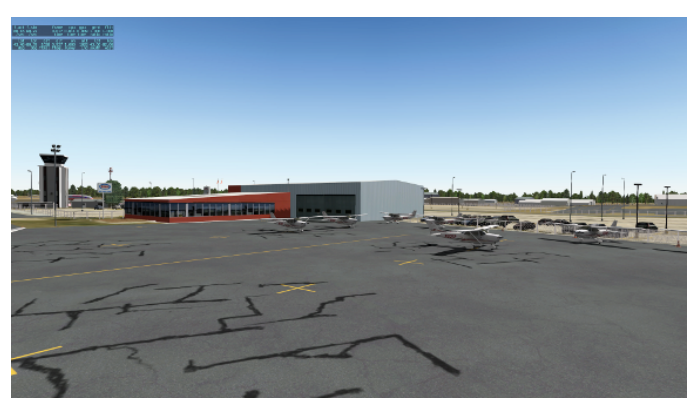

(a) WWFC buildings as seen from the apron.

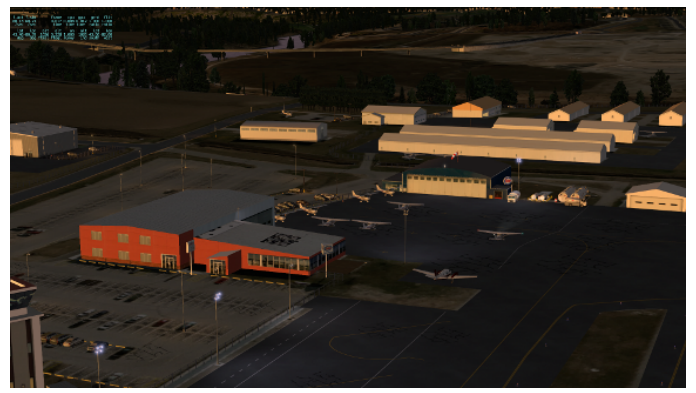

(b) WWFC buildings at sunset

Figure 7.1: Screenshots from the custom scenery for airport CYKF showing the WWFC operation. Images by Hooman Rajaee.

If desired, the instructor may sit in the right-hand seat instead of at the instructor station PC. In this case, an Apple iPad tablet computer running the official X-Plane "Control Pad" app by Laminar Research is used to set up and control the simulation session [141]. The iPad is connected wirelessly to the X-Plane LAN in order to communicate with the master PC. Using the iPad app increases training realism by allowing the instructor to sit beside the student, as would be the case if training in an actual Cessna 172 aircraft. As iPads are becoming increasingly common in general aviation to run electronic flight bag (EFB) apps, the presence of a tablet in 
the cockpit does not detract from simulation fidelity.

Since the cockpit of CURVS is type-specific to a Cessna 172, the student may execute all checklists as if in the actual aircraft. In contrast to other flight training devices, the asymmetric field of view offset toward the pilot's side allows the movement of the aileron and flap on the left wing to be checked as part of the pre-flight checks, just as in the actual aircraft.

The state of the cockpit switches for the ignition, lights, fuel tank selector, etc. are read by a LabJack T7 DAQ, which is polled regularly over its Ethernet connection by the CURVS Digital Input Interface Program. Current switch positions are then passed to X-Plane via the open-source NASA X-Plane Connect plugin.

The state of the analogue cockpit controls such as the yoke, rudder pedals, throttle, mixture, brakes, etc. are read by a separate LabJack T7 DAQ which is in turn polled regularly by the CURVS Analogue Input and Control Loading Interface Program. Current control positions are then passed to X-Plane via the X-Plane Connect plugin. Using this plugin, data is also requested from X-Plane in order to run the CURVS control loading motors. The calculated pitch, roll, and yaw forces are translated into \pm 10 VDC command voltages, which are in turn generated by two LabJack LJTick-DAC analogue output modules connected to the same LabJack T7 DAQ. These command voltages are applied across the CMND+ and CMND- terminals on the control loading motor drives in order to cause the appropriate output torques to be applied to the flight controls.

The respective states of the servo-driven flight instruments are controlled by the CURVS Servo Instrument Interface Program. Current instrument readings are requested from X-Plane via the X-Plane Connect plugin. Unlike the previous two interface programs, which communicated via Ethernet with LabJack T7 DAQs, the servo instrument interface program communicates with a dedicated hobby servomotor control board via a USB-to-Ethernet adapter. The appropriate servo pulsewidths 
corresponding to the current instrument readings are calculated by the interface program before being passed to the servo control board as specially-encoded bytes via a virtual serial port.

All three interface programs run on the master PC, which also serves as an instructor station. The master PC communicates with the three image generation PCs via an Ethernet LAN.

Out-the-window visuals are provided by three high-definition home theatre projectors. Each projector is connected to a dedicated image generation PC, each of which is slaved to the master PC. Custom view offsets are used to provide distinct left, centre, and right views. The virtual camera eyepoint is set to coincide with the pilot's seat. Since the eye point is not currently adjustable between pilot and copilot, some perspective error is present in the view from the copilot's seat. However, as CURVS is intended primarily for single-pilot ab-initio flight training as opposed to crew training, this compromise was deemed acceptable.

The Immersive Display PRO geometric correction and soft-edge blending application is used on the image generation PCs to correct for the curve of the projection screen and ensure that the overlap between projected images is not visible. The Immersive Display PRO client application runs automatically on the bootup of the image generation PCs.

Failures of aircraft systems may be triggered from within X-Plane, using either the instructor station, or the iPad app. The instruments in CURVS are driven from datarefs which represent the indicated values of the instruments. This allows the physical instruments in the cockpit to respond appropriately to simulated system or instrument failures.

Audio feedback is provided by a 5.1-channel speaker system, while tactile feedback is provided by Buttkicker ${ }^{\circledR}$-brand vibration transducers attached to the pilot's and copilot's seats. Although both speakers and vibration transducers are driven by the 
same audio signal, the frequency response of each type of device is different. This allows the vibration transducers to fill in the low-frequency range which the speakers are unable to cover. The addition of vibratory feedback increases the fidelity and immersivity of the simulation, allowing the student to feel intuitively whether the aircraft is rolling on the runway or flying just above it, in addition to sensing engine RPM.

Finally, simulated radio modules and a simulated Garmin GNS 530W permit radio and GPS navigation procedures to be performed. The commercial simulated GPS acts as a second monitor, and is connected to a dedicated X-Plane PC on the simulation network. The GPS computer is thus kept in sync with the master PC, ensuring that the location shown on the simulated GPS unit matches the position of the aircraft. The simulated GPS is shown in Figure 2.5.

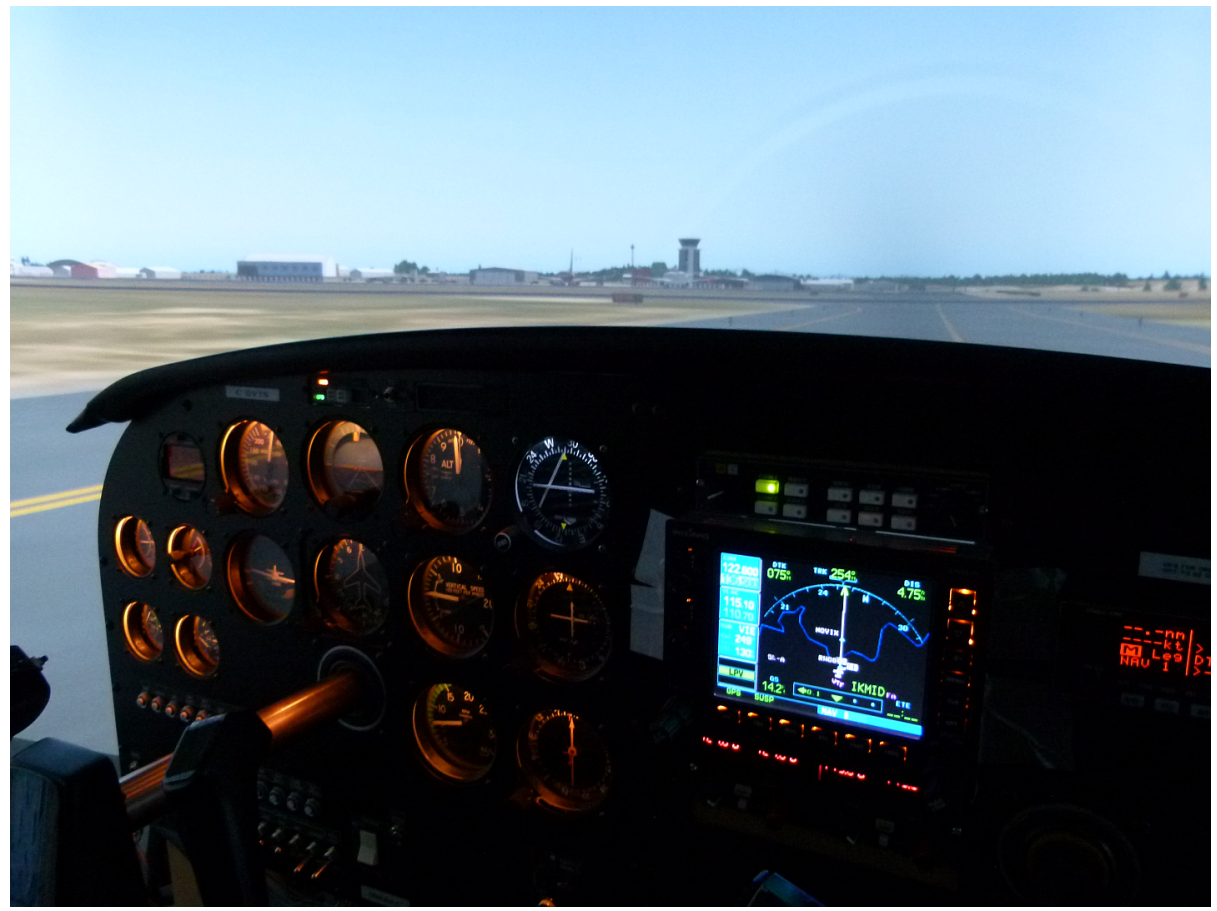

Figure 7.2: The cockpit of CURVS just after a simulated landing at CYKF. Note the simulated GPS and mechanical flight instruments. 


\subsection{CURVS Shutdown}

At the conclusion of a simulation session, the CURVS FTD is powered off by following the shutdown checklist given in Table 7.3. Note that for safety reasons, the control loading system is always disabled when not specifically in use.

Table 7.3: CURVS shutdown checklist.

\begin{tabular}{|l||l|}
\hline Challenge & Response \\
\hline Control loading & DISABLE (E-stop button DOWN) \\
\hline X-Plane > Local Map > Shutdown All & CLICK \\
\hline Projectors & OFF (use remote control) \\
\hline Buttkicker & OFF (verify light OFF) \\
\hline Simulator power & $\begin{array}{l}\text { OFF (red rocker switch behind pilots } \\
\text { seat) }\end{array}$ \\
\hline Simulator power bar & OFF \\
\hline LabJack power bar & OFF \\
\hline
\end{tabular}




\section{Chapter 8}

\section{Conclusion}

\subsection{System-by-system Assessment}

\subsubsection{Analogue Input System}

The analogue input system from the original VectorSim was successfully reverseengineered, reused, and interfaced to X-Plane using a LabJack T7 DAQ, custom C++ code, and the XPC plugin. A custom patch cable was created to convert between the existing VectorSim connector pinout and that featured on the T7 DAQ. The original linear slide potentiometers used on the pitch, roll, and yaw axes were found to suffer from spurious readings or "noise." While it was possible to temporarily rehabilitate the sensors using a potentiometer cleaning solution, the noise tended to return over time. This indicated that the potentiometers were nearing the end of their useful life and would require replacement. Exact replacements for the original slide potentiometers were found to be unavailable, however; rotary string potentiometers are readily available and would make an acceptable substitute. 


\subsubsection{Analogue Input Calibration}

A custom calibration program was developed in order to automatically record the maximum and minimum voltages produced by the analogue input system. The ability to calibrate the analogue inputs automatically by simply starting a program and moving the analogue controls through their complete ranges of motion was a major improvement over the labour-intensive, manual calibration process originally used on the VectorSim which required two people. The automatic calibration program eliminated the issue of potentiometer drift, and will allow potentiometers to be replaced and recalibrated as they wear out with a minimum of effort.

\subsubsection{Image Generation Computers}

The original flight simulation and image generation PCs used in the original VectorSim were completely replaced with four purpose-built high-performance gaming PCs using COTS parts exclusively. The PCs were designed for cost-effective longterm performance, and have been verified to reliably run X-Plane on high graphical settings at 60 FPS.

\subsubsection{Control Loading}

The control loading system in the original VectorSim was successfully reverseengineered and interfaced to X-Plane using a LabJack T7 DAQ, two LJTick-DAC analogue output modules, custom $\mathrm{C}++$ code, and the $\mathrm{XPC}$ plugin.

In addition, an innovative, iterative method for tuning X-Plane stick force output to match available flight test data was successfully developed. This alternate approach was necessary due to the fact that the specific flight test data required by X-Plane in order to enable control loading is not typically gathered during FTD qualification flight testing. This innovative method was based entirely on pre-existing flight test 
data, and was able to determine plausible values of the data required by X-Plane without resorting to actual flight tests.

\subsubsection{Servo-based Flight Instruments}

The pre-existing servo-based flight instruments were successfully reverse-engineered and interfaced to X-Plane using three custom patch cables, a Pololu Mini Maestro 24-channel servo controller, custom $\mathrm{C}++$ code, and the XPC plugin. Total cost of new parts required was approximately $100 \mathrm{CAD}$.

A piecewise-based linear interpolation algorithm was developed in order to accommodate the many non-linear instrument scales which were featured in the original servo-based flight instruments. In addition, the accuracy of the airspeed indicator was improved by increasing the number of calibration points from the original six to ten.

The new instrument interface program featured a user-modifiable calibration file which mapped key values on the instruments to the corresponding servo pulsewidths required to obtain those indications. The use of a separate calibration file external to the interface code allows any damaged servos to be quickly replaced by the end-user without recompilation of any code. Where necessary, the calibration file also recorded the pulsewidths corresponding to mechanical endstops within the instruments. This allowed the interface program to perform "sanity checks" on the interpolated servo pulsewidths to ensure that they did not exceed the physical range of the instruments. Driving a servo into an instrument endstop can result in stripped gears, rendering the servo useless. 


\subsubsection{GPS}

A simulated modern GPS was successfully implemented using an additional X-Plane PC running a third-party GPS simulation as a plugin. This approach successfully overcame the inability of X-Plane plugins to be "undocked" from the main X-Plane window for display on a separate monitor, such as the one in the simulated GPS unit. As high 3D graphics performance was not required for the GPS plugin, cost was kept to a minimum by repurposing a surplus PC obtained without cost following a departmental computer upgrade and running X-Plane on minimum graphical settings. CURVS now features a simulation of a modern, fully-graphical Garmin GNS 530W aviation GPS unit instead of the dated Bendix-King KLN-89B GPS unit originally installed.

\subsubsection{Digital Input}

Due to errors discovered in the original wiring lists, reverse-engineering of the original circuitry for the digital input system was found to be too time-consuming and was abandoned. The existing switch wiring was cut, and the switches interfaced directly to the digital inputs of a LabJack T7 DAQ. The internal pull-up resistors built into the DAQ meant that switches in the physical off position were registered by the DAQ as logic level HIGH. While confusing from a common-sense perspective, the switches were nevertheless successfully interfaced to X-Plane. When cutting the original wiring harness, it was discovered that the taxi light switch had never been connected in the original VectorSim. This oversight was corrected, and the taxi light now operates as intended. 


\subsubsection{Radio Stack}

The original, proprietary simulated radio stack was removed, and is in the process of being replaced with simulated radio modules. Unlike the original units, these new modules closely approximate the look of the actual Bendix-King radio equipment used in the WWFC fleet. The original DME and second NAV/COM radio modules were removed and not replaced due to the fact that equivalent functionality was provided by the new simulated GPS mentioned above. These upgrades will allow CURVS to match the WWFC fleet much more closely than before, and thus improve transfer-of-training between the simulated environment and the actual aircraft.

\subsubsection{Supporting Systems}

\section{Projection}

The new, $220^{\circ}$ asymmetric projection screen is a vast improvement on the single lowresolution projection screen originally installed. The new projection screen allows a full $180^{\circ}$ horizontal field of view from both seats, while an additional $40^{\circ}$ field of view on the pilot's side allows the pilot to look back over their left shoulder. This asymmetric projection system favouring the pilot is unique among commercially available FTDs, and allows the pilot to maintain superior situational awareness when flying the left-hand aerodrome circuit commonly used in modern aviation. This innovation allows left-hand circuits to be flown entirely by visual reference to the ground, without requiring any cues from a flight instructor with access to the moving map display at the instructor operator station.

\section{Custom Scenery}

The new, custom X-Plane scenery developed for CURVS is a major improvement on the circa 2002 computer graphics used in the original VectorSim. In fact, the visual 
detail available at airport CYKF and in the surrounding areas surpasses that of the modern, commercial FTD currently in use at WWFC, a device which cost approximately 500,000 CAD. The CURVS scenery at CYKF features accurate placement of all buildings, with custom 3D models being used for the control tower, airport terminal building, and WWFC buildings. Pavement markings, taxiway signage, and airport lighting are all true-to-life. As per the Transport Canada preference for FTDs, working models of all windsocks installed at CYKF were also included. Satellite background imagery was used to augment the runway and taxiway geometry, resulting in a near-photorealistic effect.

\section{Audio}

The AKAI S2000 digital sampler used to produce sound effects in the original VectorSim was removed, and replaced with sound effects generated by X-Plane. In addition, the pair of 2-watt PC speakers originally installed were removed, and replaced with a more powerful 75-watt COTS speaker system consisting of five small speaker modules and a subwoofer. To improve audio fidelity, the default X-Plane sound effect files were replaced with high-fidelity audio recordings from an actual Cessna 172. These new sound effects featured noticeably improved bass frequency content, and greatly improved the realism of the simulation compared with the actual aircraft.

\section{Vibration}

Realism of CURVS was further increased compared with the original VectorSim by the addition of COTS seat vibration transducers. These transducers served to fill in the low-frequency region below the frequency response of the upgraded speaker system. These transducers allowed the pilots to intuitively sense engine RPM, as well as the exact moment of contact between the tires and the runway, an event which can be difficult to discern in the absence of a motion platform. Engaging the human sense 
of touch in addition to vision and hearing resulted in a more immersive simulation which was more representative of an actual Cessna 172, and resulted in improved situational awareness for the pilots.

\subsection{Cost Analysis}

The projected cost of parts and software licences for the completed CURVS FTD is approximately 30,000 CAD. The cost of graduate student research assistantships over the course of two years was defrayed by means of governmental research grants, resulting in no net monetary cost to WWFC for salaries. The residual value of the existing obsolete Vector simulator before redevelopment was estimated to be approximately 135,000 CAD. By the end of the CURVS project, it is anticipated that WWFC will possess a modern, type-specific Level 5 FTD which is representative of their training fleet and features control loading and a superior visual system for a total additional investment of approximately 30,000 CAD.

While "low-cost" Cessna 172 FTDs such as the Redbird SD (approximately 68,000 CAD) or the TRC 472-FG (approximately 80,000 CAD) are available, these FTDs often lack key functionality provided by the CURVS FTD such as control loading or its innovative, $220^{\circ}$ asymmetric wraparound projection system $[142,143]$. Instead, the features, fidelity, and planned certification level offered by CURVS allow it to be compared more directly with high-end FTDs such as the Alsim ALX which cost roughly an order of magnitude more than the redevelopment cost associated with CURVS. Thus, the redevelopment of an existing FTD as opposed to purchase of a new one is projected to result in a cost savings to WWFC of several hundred thousand dollars while also producing an FTD with superior functionality. 


\subsection{Contributions}

The author's research resulted in the design and development of a number of costeffective technologies for interfacing physical cockpits and their associated sensors and equipment to the X-Plane flight simulation program. While implemented specifically on the CURVS FTD, these technologies are modular and reusable, and may be used in the creation or redevelopment of any aeroplane or rotorcraft simulator. These technologies are itemized below.

- Analogue input. A robust, cost-accessible (400 USD) analogue-input system consisting of COTS hardware and custom interface software was designed, developed, and implemented. This system is capable of reading up to 14 potentiometers or other analogue sensors, although only 13 are required in CURVS. The accompanying software may be easily modified to allow any analogue input to drive any X-Plane software function, e.g., stick, throttle, or flap positions. This allows the analogue input system to be easily adapted for use in any flight simulator. Individual calibration values for each input channel are also supported. This allows sensors to be easily replaced - even with sensors of a different type - without recompiling the interface software.

- Analogue input calibration. An automatic calibration program was designed and developed to work in conjunction with the analogue input system. This program allows the analogue inputs of the CURVS FTD to be calibrated by a single person, as opposed to the two which were previously required. The calibration text file produced by the program is human-readable, allowing its contents to be examined for troubleshooting purposes if desired. This program may be easily adapted for use with any flight simulator which also uses the analogue input system described above. 
- Simulation PCs. A high-performance yet cost-accessible simulation/imagegeneration PC architecture was designed, implemented, and tested in the CURVS FTD. This PC design was verified to be capable of running the X-Plane software at a constant 60 FPS at near-maximum graphical settings in conjunction with complex add-on scenery. Use of high-end yet commonly-available COTS components enabled an affordable cost-per unit of just 1700 CAD. This high-performance PC design may now be reused in any new simulation project with no additional investment in research and development time.

- Control Loading. A robust, cost-accessible (560 USD) control loading interface system consisting of COTS hardware and custom interface software was designed, developed, and implemented. Use of manufacturer-specific control protocols was avoided, meaning that this system is adaptable to any existing control loading system whose motor controllers are capable of analogue input. In addition, a method for quantifying the relationship between analogue input voltage and resulting output force was developed. This method does not require knowledge of linkage geometry, and is thus applicable to any new or pre-existing simulator.

- Control Loading Calibration. An easy-to-use, iterative method for tuning control force output to match standard Transport Canada flight test data was developed and successfully implemented for the pitch axis. Use of this method eliminated the need to perform the unique, non-standard flight tests required by X-Plane. This method is applicable to any fixed-wing flight simulator which uses X-Plane's calculated control force values and for which existing simulator qualification data is available. As such, this method is ideal for the redevelopment and modernization of other currently-obsolete flight simulators which might otherwise be discarded. 
- Servo Instrument Interface. A cost-effective (100 CAD) interface between X-Plane and physical, servo-driven simulated flight instruments was developed and successfully implemented. Designed to control 18 hobby servomotors, the associated software may be easily modified to support up to 25 servomotors, thus allowing this technology to be quickly adapted for use with any flight simulator which uses X-Plane. If more than 25 servo-driven items of simulated instrumentation are required, a second system may be operated in parallel with the first.

- Asymmetric Cylindrical Projection. A cost-effective cylindrical projection system was designed and implemented under the author's guidance. This system featured a novel, $40^{\circ}$ view offset on the pilot's side which greatly enhanced pilot immersion and situational awareness. This innovative display system may be easily adapted for use in future aeroplane or rotorcraft simulators.

The development of the aforementioned technologies has shown that it is possible to reduce the cost barrier which currently impedes the adoption of flight simulation in ab-initio flight training, especially at smaller flight training units. Using readilyavailable COTS hardware and software components, it has been demonstrated that it is possible to produce a cost-effective, robust, and reliable flight simulation environment suitable for use in ab-initio flight training.

In addition, it has been shown that the prohibitive expense of existing COTS control loading solutions can be reduced by reusing components such as brushless servomotors and servomotor drives commonly considered to be obsolete but which are in fact still serviceable. Such systems may be returned to service with only a modest investment in modern, cost-effective yet robust interface hardware such as the LabJack T7 DAQ. 


\subsection{Future Work}

Several tasks remain in order to complete the redevelopment of the existing FTD and prepare it for commercial use at WWFC. In order of importance:

1. Improve the stability of the control loading system. The force loop iteration rate should be increased to a minimum of $500 \mathrm{~Hz}$, as discussed in Chapter 5 . In addition, delays should be introduced in the digital input and servo instrument interface programs in order to prevent the XPC plugin from being overloaded, and thus causing all of the various interface programs to slow down. Since the $\mathrm{XPC}$ plugin is only run once per X-Plane simulation cycle, communication with the plugin should always occur at a rate less than or equal to the X-Plane frame rate.

In the event that a faster control force iteration rate is unable to stabilize the control loading system, software damping should be implemented as part of the external control loading algorithm. Damping should only be activated above a certain threshold value, however. This will prevent the damping component of the algorithm from interfering with the pilot's normal movement of the controls. As numerical differentiation is prone to measurement noise, position feedback from the potentiometers should first be processed with a low-pass filter (LPF) to remove high-frequency noise spikes. This rate-trigged damping scheme is illustrated below:

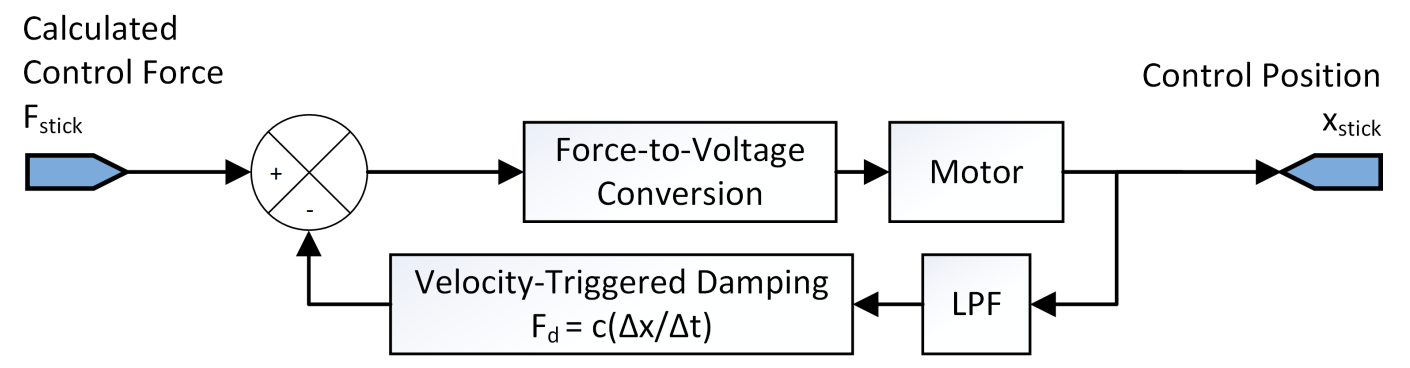


2. Complete the LabJack interface program for the stepper-driven flight instruments. Similar to the servo instrument interface program, the stepper instrument interface program must poll XPC regularly for new values, then increment or decrement the four stepper motor instruments by the appropriate number of steps. This program must also include a start-up calibration routine in order to position the altimeter, $\mathrm{ADF}$, and magnetic compass at the correct initial positions. As the indication on the directional gyro is always relative, no initial calibration is required for this instrument.

3. Interface to the existing circuit breaker subsystem. While reverse-engineering of the existing system is nearly complete, a LabJack interface program is still required. This program shall poll the XPC plugin at intervals to determine the current state of the simulated breakers in X-Plane. The program shall also report the current physical state of the breakers in order to determine if any breakers have been reset by the pilot. Since breaker trips and resets typically occur infrequently, an update frequency of $10 \mathrm{~Hz}$ should be more than sufficient for this program.

4. Interface to the original "digital output interface" card. This circuit board of Darlington switches enables the Cessna annunciator and electrical relay subsystem. The annunciator provides warning lights for certain aircraft systems, while the relay system allows master control over the electrical systems in the FTD, thus allowing electrical failures to be simulated.

5. Implement an intercom system so that headsets may be used. The original VectorSim featured a working intercom as part of the simulated radio stack. However, as the proprietary radio stack was replaced, a new intercom system is required. A spare aircraft intercom unit is available, although some additional research will be required in order to inject X-Plane audio into the loop. 


\subsection{Epilogue}

An initially-obsolete, non-functional Cessna 172 FTD by the former Vector Training Systems, Inc. was reverse-engineered, redeveloped, modernized, and its capabilities greatly extended to produce CURVS, the Carleton University Redeveloped Vector Simulator, shown in Figure 8.1. This new FTD, suitable for use in ab-initio as well as instrument flight training, is nearly complete and will be returned to active service with WWFC at airport CYKF in Canada.

Throughout this work, the use of pre-existing COTS hardware and software was emphasized, with priority being given to solutions which were both cost-effective and robust in the context of long-term use in a professional flight training environment. The redevelopment work was completed for less than 30,000 CAD in new parts and software, not including labour. Thus, the CURVS project has demonstrated that fidelity, immersivity, and cost-effectiveness are not mutually exclusive, and that the potential exists for significant reductions in the cost of high-quality flight simulation for use in ab-initio flight training. 


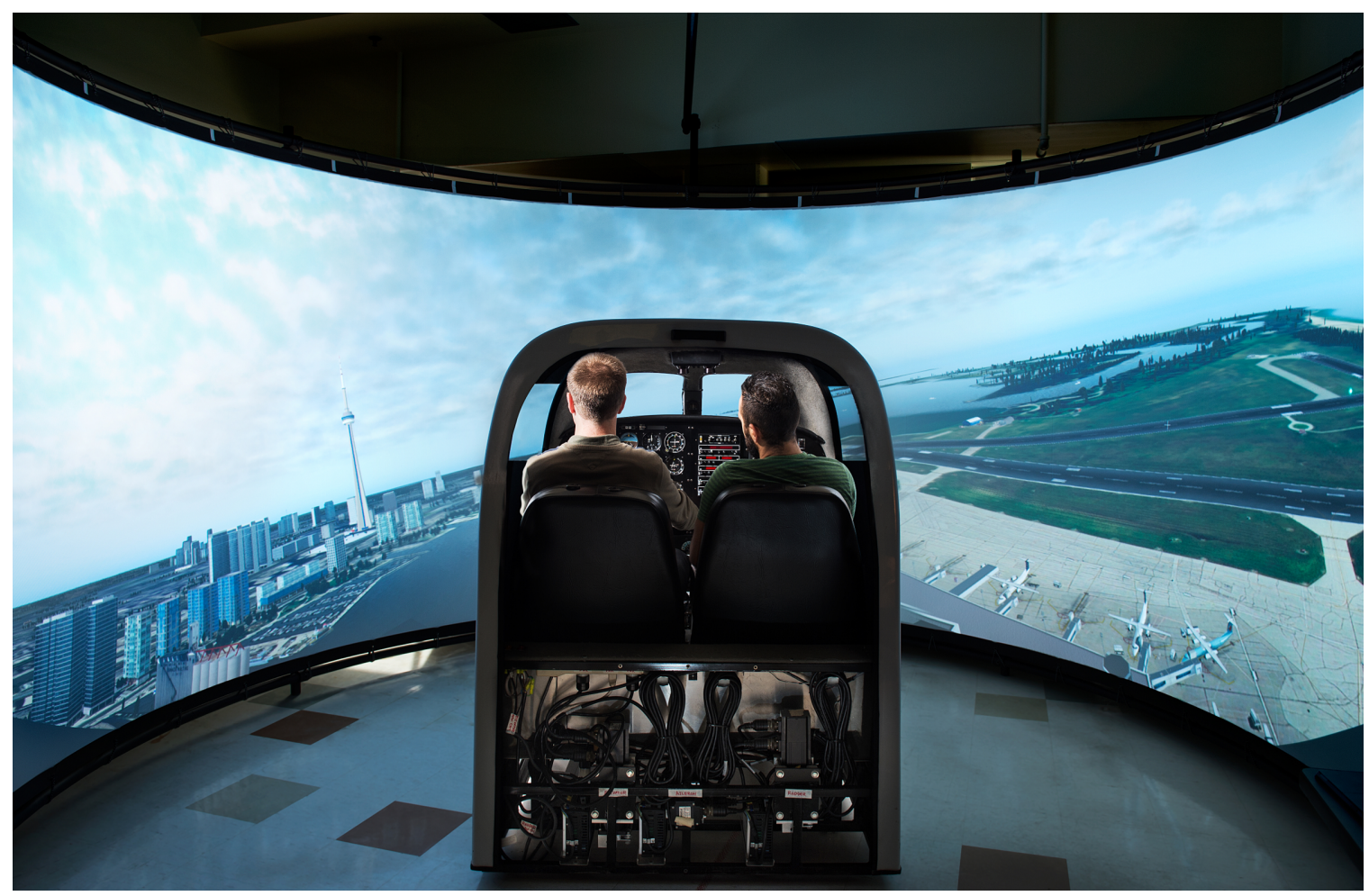

Figure 8.1: The CURVS FTD, in flight over the city of Toronto, Canada. Image courtesy of Luther Caverly. 


\section{References}

[1] Transport Canada. Aeroplane and Rotorcraft Simulator Manual, TP9685E, 1998.

[2] International Civil Aviation Organization, Doc 9625. Manual of Criteria for the Qualification of Flight Simulation Training Devices, 3rd edition, 1999.

[3] Federal Aviation Administration. Airplane Flight Training Device Qualification, $A C$ 120-45A, 1992.

[4] Federal Aviation Administration. Airplane Simulator Qualification, AC 120$40 B, 1991$.

[5] ALSIM ALX simulator: all your aircraft in one device. Available: http://www . alsim.com/simulators/alx/. Accessed: 2016, March 25.

[6] Precision Flight Controls Homepage. Available: http://www.flypfc.com/. Accessed: 2016, March 25.

[7] Vector Training Systems, Inc. Victory Two Single-Engine Land Level 3 Flight Training Device Project P-7600 Reference Data Report, 2002.

[8] WWFC Aircraft \& Rates. Available: http://www.wwfc.ca/fleets-ratesaircrafts/. Accessed: 2016, March 25.

[9] Jonathan Plumpton. Development of a full-flight simulator for ab-initio flight training with emphasis on hardware and motion integration. Master's thesis, Carleton University, 2014.

[10] Suzanne Swaine. Development of a cost-effective high-fidelity type-specific flight simulator with emphasis on flight modelling. Master's thesis, Carleton University, 2014. 
[11] New Helicopter Full-Flight Simulator for the Canadian Coast Guard. Available: http://news.gc.ca/web/article-en.do?nid=1036029. Accessed: 2016, June 30 .

[12] CAE awarded contract to provide Bell 412/429 helicopter simulator to Canadian Coast Guard. Available: http://www . cae.com/CAE-awarded-contractto-provide-Bell-412-429-helicopter-simulator-to-Canadian-CoastGuard/. Accessed: 2016, June 30.

[13] Vector Training Systems, Inc. FSTEST Manual, 2000.

[14] Technical Specifications - Flight Training Devices - Vector Training Systems [Archived]. Available: https://web.archive.org/web/20001217223600/ http://vtsweb.com/ftd/schem.html. Accessed: 2016, June 4.

[15] Firmenbuch-Bekanntmachung der Firma Luftfahrtstudio Jungwirth Gesellschaft mbH. Available: http://firmenbuchauszug.at/ Firmenbuchinformation/2352FC09F9931D. Accessed: 2016, April XX.

[16] B. M. Cook, editor. Legacy of the Transputer. World occam and Transputer User Group (WoTUG), IOS Press, 1999. Available: http://www.wotug.org/ papers/IvimeyCook_W22.pdf.

[17] KJ. Catsoulis. Transputers - extinct but not forgotten. Available: https://web.archive.org/web/20040314183809/http://www . embedded. com.au/reference/transputers.html. Accessed: 2016, April 9.

[18] D. Ellison. Understanding occam and the Transputer - through complete working programs. Sigma Press, Wilmslow, Cheshire, UK, 1991.

[19] B. Vinter. Transputer Architecture. Available: http://www.diku.dk/ vinter/xmp/lecture6Transputer .pdf. Accessed: 2016, April 9.

[20] General Information - Flight Training Devices - Vector Training Systems [Archived]. Available: https://web.archive.org/web/20001218015900/ http://vtsweb.com/ftd/sound.html. Accessed: 2016, June 4.

[21] D. Hodges. Darlington's contributions to transistor circuit design. IEEE Transactions on Circuits and Systems I: Fundamental Theory and Applications, 46(1):102-104, 1999. Available: http://www.eecs.berkeley.edu/ hodges/ DarlingtonCircuit.pdf. 
[22] How X-Plane Works. Available: http://www.x-plane.com/desktop/how-xplane-works/. Accessed: 2016, June 28.

[23] Longitudinal stability derivatives. Available: http://aerostudents. com/files/flightDynamics/longitudinalStabilityDerivatives.pdf. Accessed: 2016, June 28.

[24] H. Loewen. Stability Derivatives: What they are and how they are used. Available: https://www.micropilot.com/pdf/stability-derivatives.pdf. Accessed: 2016, June 28.

[25] About the Aces Team. Available: https://www.microsoft.com/Products/ Games/FSInsider/news/Pages/AMessageFromAces.aspx. Accessed: 2015, January 16.

[26] Microsoft Flight Simulator X: Steam Edition. Available: http://store. steampowered.com/app/314160/. Accessed: 2016, March 28.

[27] FSX Insider: FAQs. Available: https://fsxinsider.com/faqs/. Accessed: 2015, January 16.

[28] Lockheed Martin Launches Prepar3d TM to Provide Richer Training Experience for Warfighters. Available: https://web.archive.org/web/ 20100713125615/http: //www. lockheedmartin.com/news/press_releases/ 2010/05172010Prepar3d.html. Accessed: 2016, July 7.

[29] Fidelity Flight Simulation, Inc. Homepage. Available: http://www.f2si.net/ index.html. Accessed: 2016, June 28.

[30] FlightGear Flight Simulator. Available: http://www.flightgear.org/. Accessed: 2016, March 29.

[31] The CASARA Virtual Trainer. Available: http://carleton.ca/ace/ projects-and-publications/casara-simulation-training/. Accessed: 2016, March 29.

[32] Scott Arbuthnot (Senior Simulation Specialist, Advanced Cognitive Engineering Laboratory, Carleton University). Personal correspondence, March 2016.

[33] FixedWingLib FDM for C++. Available: www.rtdynamics . com/v3/productsservices/simulation-and-training/fixedwinglib-fdm-for-c/. Accessed: 2016, March 30. 
[34] Multiple Monitor (over/under) setup. Available: http://web. archive.org/web/20150726024117/http://www . prepar3d.com/forum5/topic/multiple-monitor-overunder-setup/. Accessed: 2015, January 16.

[35] Official FSUIPC Download Page. Available: http://www.schiratti.com/ dowson.html. Accessed: 2016, June 29.

[36] Prepar3D SimConnect. Available: http://www.prepar3d.com/SDKv2/ LearningCenter/utilities/simconnect/simconnect.html. Accessed: 2016, June 29.

[37] Laminar Research. Sending Data to X-Plane.html.

[38] X-Plane Plugin SDK User's Manual. Available: http://www .xsquawkbox.net/ xpsdk/mediawiki/Category:Documentation. Accessed: 2016, April 19.

[39] XPlaneConnect. Available: https://github.com/nasa/XPlaneConnect. Accessed: 2016, April 19.

[40] R\&R Electronics. R\&R Electronics - Epic Card. Available: www.mindspring. com/ rrelect/. Accessed: 2016, April 5.

[41] Johnny A. Martin. The Extended Programmable Input/Output Controller (EPIC) Developer's Manual, June 2003. Accessed: 2014, July 10.

[42] Leo Bodnar Electronics. Universal USB Interface Boards. Available: http: //www. leobodnar.com/shop/index.php?main_page=index\&cPath=94. Accessed: 2016, April 5.

[43] Inc. Phidgets. I/O Boards. Available: http://www.phidgets.com/products . php? category=0. Accessed: 2016, April 5.

[44] Jan Axelson. USB Complete: Everything You Need to Develop Custom USB Peripherals, page 3. Lakeview Research LLC, Madsion, WI, 3rd edition, 2005.

[45] Arduino LLC. Arduino Products. Available: https://www.arduino.cc/en/ Main/Products. Accessed: 2016, April 05.

[46] Arduino LLC. Arduino Ethernet Shield. Available: www .arduino.cc/en/Main/ ArduinoEthernetShield. Accessed: 2016, April 05. 
[47] S. Swaine, J. Plumpton, J. Leveille, and R. Langlois. Feasibility assessment of a cost-effective type-specific small aircraft flight simulator. In Proc. Canadian Society for Mechanical Engineering (CSME) Forum, June 2012. Winnipeg, Canada.

[48] Vlad Sychev. Project Baron 58 - Home Cockpit Simulator for X-Plane. Available: http://b58.svglobe.com/. Accessed: 2016, March 31.

[49] John D. Hayes. Carleton University Simulator Project. Available: http://carleton.ca/mae/wp-content/uploads/2016-MAE-CarletonUniversity-Simulator-Project.pdf. Accessed: 2016, March 31.

[50] Raspberry Pi Foundation. Raspberry Pi 2 Model B. Available: https://www . raspberrypi.org/products/raspberry-pi-2-model-b/. Accessed: 2016, April 05.

[51] BeagleBoard.org Foundation. BeagleBone Black. Available: http:// beagleboard.org/black. Accessed: 2016, April 05.

[52] Gerald Coley. BeagleBone Black System Reference Manual. BeagleBoard.org, May 2014. Revision C.1.

[53] Pololu Corporation. RC Servo Controllers. Available: https://www pololu . com/category/12/rc-servo-controllers. Accessed: 2016, April 15.

[54] Pololu Corporation. Stepper Motor Drivers. Available: https://www pololu . com/category/120/stepper-motor-drivers. Accessed: 2016, April 15.

[55] T7 Series. Available: https://labjack.com/products/t7. Accessed: 2016, April 14.

[56] LJTick-DAC. Available: https://labjack.com/accessories/ljtick-dac. Accessed: 2016, April 14.

[57] BeagleBoard.org Foundation. Official BeagleBone Black Wiki: Improper Power Down....All Revisions. Available: http://elinux.org/Beagleboard: BeagleBoneBlack\#Improper_Power_Down....All_Revisions. Accessed: 2016, April 14.

[58] J. Wilhelmi. Personal correspondence. Email, August 2015.

[59] Simulator Maintenance Technicians (SIM). Available: http://www.Irsc. edu/academics/programs/simulator-maintenance-technicians. Accessed: 2016, April 18. 
[60] Foxit Reader 7 - The Secure PDF Reader. Available: https://www . foxitsoftware.com/products/pdf-reader/. Accessed: 2016, April 18.

[61] Teensy USB Development Board. Available: https://www.pjrc.com/teensy/ index.html. Accessed: 2016, April 18.

[62] Matrox TripleHead2Go Analog Edition. Available: http://www.matrox.com/ graphics/en/products/legacy/gxm/th2go/analog/. Accessed: 2016, April 18.

[63] Solving Instrumentation Ground Loop Problems. Available: http: //ecmweb.com/bonding-amp-grounding/solving-instrumentationground-loop-problems. Accessed: 2016, May 28.

[64] Arduino - Map. Available: https://www.arduino.cc/en/Reference/Map. Accessed: 2016, April 19.

[65] M. Greer. Why system() is evil. Available: http://www.cplusplus.com/ articles/j3wTURfi/. Accessed: 2016, April 19.

[66] What Is a View Frustum? Available: https://msdn.microsoft.com/enus/library/ff634570. aspx. Accessed: 2016, May 3.

[67] Nikola Gidalov. MultiView X-Plane plug-in v2.0. Available: http: //forums.x-plane.org/index.php?/files/file/20524-multiview-xplane-plug-in/. Accessed: 2016, May 3.

[68] MultiView X-Plane Plug-in v.2.0 User Guide. Digital download, available: http://forums.x-plane.org/index.php?/files/file/20524multiview-x-plane-plug-in/. Accessed: 2015, January 11.

[69] N. Gidalov. Personal correspondence. 2015, April 2.

[70] A. Fabian. X-Plane 10 HD Mesh Scenery v3. Available: http://www . alpilotx.net/downloads/x-plane-10-hd-mesh-scenery-v3/. Accessed: 2015, April 28.

[71] System Requirements for X-Plane 10. Available: http://www.x-plane.com/ desktop/system-requirements/. Accessed: 2015, February 26.

[72] C. Angelini. Intel \& AMD Processor Hierarchy. Available: http://www . tomshardware.com/reviews/cpu-hierarchy,4312.html, November 2015. Accessed: 2016, May 4. 
[73] Silicon Dies: Explaining OC Editions \& the Bin-Out Process - CPUs, RAM, GPUs. Available: http://www.gamersnexus.net/guides/1140-silicondie-bin-out-process-explained. Accessed: 2016, May 4.

[74] Freezer i11 Compact Performance CPU Cooler. Available: https://www . arctic.ac/worldwide_en/freezer-i11.html. Accessed: 2016, May 5.

[75] MX-4 Thermal Compound for All Coolers. Available: https://www.arctic. ac/worldwide_en/mx-4.html. Accessed: 2016, May 4.

[76] SABERTOOTH Z97 Mark 2. Available: http://www.asus.com/ca-en/ Motherboards/SABERTOOTH_Z97_MARK_2/. Accessed: 2015, March 17.

[77] EVGA GeForce GTX 960 4GB SSC GAMING ACX 2.0+. Available: http: //www . evga . com/Products/Specs/GPU . aspx?pn=DFD1EEE3-99104DA3-AC8B-CA9CE52B7B4F. Accessed: 2016, May 5.

[78] G-750. Available: https://seasonic.com/product/g-750/. Accessed: 2016, May 5.

[79] D. Woligroski. Power Supply 101: A Reference Of Specifications. Available: http://www.tomshardware.com/reviews/power-supplyspecifications-atx-reference,3061-6.html. Accessed: 2016, April 16.

[80] 3DMark. Available: http://www.futuremark.com/benchmarks/3dmark. Accessed: 2016, May 3.

[81] Perfmonitor2. Available: http://www.cpuid.com/softwares/perfmonitor2.html. Accessed: 2016, May 12.

[82] Intel Core i5-4690K Processor (6M Cache, up to $3.90 \mathrm{GHz}$ ). Available: http://ark.intel.com/products/80811/Intel-Core-i5-4690KProcessor-6M-Cache-up-to-3_90-GHz. Accessed: 2016, May 4.

[83] Momentum Effects on Aerodynamic Forces. Available: https://www.grc. nasa.gov/www/k-12/airplane/momntm.html. Accessed: 2016, March 3.

[84] D. Allerton. Principles of Flight Simulation, pages 22,23. Wiley, Chichester, West Sussex, UK, 2009.

[85] F. Lutze. Stick Free and Stick Force Characteristics. Virginia Tech.

[86] F. Lutze. Complete Vehicle Lift and Pitch-Moment Equations. Virginia Tech. 
[87] J. Etele. AERO 4308 Aircraft Stability and Control Course Notes. Carleton University, 2013.

[88] Cessna Aircraft Company. Model 172S Pilots's Operating Handbook and FAA Approved Airplane Flight Manual, July 1998. Accessed: 2015, May 2.

[89] Part V - Airworthiness Manual Chapter 523 - Normal, Utility, Aerobatic And Commuter Category Aeroplanes. Available: https://www.tc.gc.ca/eng/ civilaviation/regserv/cars/part5-standards-523-sub-ab-2061.htm.

Accessed: 2016, May 13.

[90] Advisory Circular AC 120-45A. Available: http://rgl faa.gov/Regulatory_ and_Guidance_Library/rgAdvisoryCircular.nsf/list/AC\%2012045A/\$FILE/AC120-45A.pdf. Accessed: 2016, May 13.

[91] Thomson Motion Control Division. OMNIDRIVE Installation Manual for Models ODM-005 and ODM-005i, 1998. Accessed: 2016, February 17.

[92] DeoxIT Fader F-Series. Available: http://store.caig.com/s.nl/sc.2/ category.293/.f. Accessed: 2016, May 18.

[93] B. Supnik. Developing Plugins. Available: http://www.xsquawkbox.net/ xpsdk/docs/Overview.html. Accessed: 2016, May 20.

[94] K. Staples J. Rolfe. Flight Simulation. Cambridge University Press, Cambridge, UK, 1986.

[95] BFF modular CL system brushless motor driver. Available: http:// bffsimulation.com/BFF-FFB-System.php. Accessed: 2016, February 17.

[96] High Force High Fidelity Control Loader System - Model 400-X. Available: http://www. servos.com/servos-and-simulation-control-loaderhigh-fidelity---faa-level-a-d.html. Accessed: 2016, May 19.

[97] M. Peyrard O. Braun. Dependence of kinetic friction on velocity: Master equation approach. Physical Review E : Statistical, Nonlinear, and Soft Matter Physics, American Physical Society, 83(4):046129-1-9, April 2011.

[98] J. Malek. Servo control interface in detail. Available: https://www pololu . com/blog/17/servo-control-interface-in-detail. Accessed: 2014, Sept 6. 
[99] Mini Maestro 24-Channel USB Servo Controller (Assembled). Available: https://www.pololu.com/product/1356. Accessed: 2016, April 22.

[100] Pololu USB Software Development Kit. Available: https://www.pololu.com/ docs/0J41. Accessed: 2016, April 25.

[101] S. Burke. Cross-platform, open source .NET framework. Available: http: //www.mono-project.com/. Accessed: 2016, April 22.

[102] Installing a Debian v3.08 Linux Armhf $\mathrm{O} / \mathrm{S}$ on the Beaglebone Black. Available: http://www.ofitselfso.com/Beagle/ InstallingArmhfKernelBeagleboneBlack.php. Accessed: 2016, April 30.

[103] USB Extender over CAT5E or CAT6 Connection up to 150ft, Product \# 6042. Available: http://www.monoprice.com/product?p_id=6042. Accessed: 2016, May 2.

[104] Pololu Maestro Servo Controller Users Guide, Section 5.h.2.: Windows C. Available: https://www.pololu.com/docs/0J40/5.h.2. Accessed: 2016, March 1.

[105] M. Grant. Quick Start for Beginners to Drive a Stepper Motor. Freescale Semiconductor, Inc., June 2005. Accessed: 2015, February 5.

[106] Types of Steppers. Available: https://learn.adafruit.com/all-aboutstepper-motors/types-of-steppers. Accessed: 2016, April 30.

[107] Motorola, Inc. Motorola MC3479 Stepper Motor Driver, 1996.

[108] Honeywell. HOA2001 Transmissive Optoschmitt Sensor.

[109] GNS530. Available: http://www.emuteq.com/GNS530.html. Accessed: 2016, April 26.

[110] 400W/500W Series Trainer - WAAS software version 3.00. Available: https: //www8.garmin.com/support/download_details.jsp?id=3531. Accessed: 2016, April 27.

[111] S. Masters. General Aviation GPS Hardware Interface: GarSim GNS530. Available: http://docs.google.com/leaf?id=0B_bTMUTZ3P2MjA1MjQzZDEtYTY3MC00MGRmLWF1ZjctZGNjMWYwYTI2NmI5\&hl=en_GB. Accessed: 2016, April 26. 
[112] S. Masters. General Aviation GPS Hardware Interface. Available: http://www . mycockpit.org/forums/content.php?r=139-GeneralAviation-GPS-Hardware-Interface-tutorial. Accessed: 2016, April 26.

[113] GNS 530W XP XPlane. Available: http://www.reality-xp.com/flightsim/ gns530-xpl/index.html. Accessed: 2016, April 27.

[114] FTS430W and FTS530W. Available: http://www.flythissim.com/FTS430. html. Accessed: 2016, April 26.

[115] Navigation Data. Available: http://www.flythissim.com/FMS_data.html. Accessed: 2016, April 26.

[116] RE: FTS 430W nav database. Email, April 2016. Personal correspondence.

[117] SmartCopilot: X-Plane plugin for crew flying. Available: http://xsmartplane.com/smartcopilot/. Accessed: 2016, April 27.

[118] B. Supnik. Datarefs for X-Plane 1041. Available: http://www.xsquawkbox . net/xpsdk/docs/DataRefs.html. Accessed: 2016, March 14.

[119] Mechanical Switch Closure (App Note). Available: https://labjack.com/ support/app-notes/mechanical-switch-closure. Accessed: 2016, April 28.

[120] Pull-up Resistors. Available: https://learn.sparkfun.com/tutorials/ pull-up-resistors. Accessed: 2016, April 28.

[121] Home simulator builders startpoint. Available: https://www . flightillusion.com/. Accessed: 2016, April 27.

[122] AlliedSignal. Installation and Maintenance Manuals: BENDIX/KING KI 208A/209A Navigation Indicators, October 1996. Accessed: 2016, February 10.

[123] King KI-209A GPS / VOR / LOC / Glideslope Indicator. Available: http: //www. bennettavionics.com/ki209a.html. Accessed: 2016, February 24.

[124] Data Device Corporation. Synchro/Resolver Conversion Handbook, 4th edition, 1994. Accessed: 2016, February 23.

[125] GSA-058 VOR-1 with Glideslope indicator. Available: https: //www.flightillusion.com/product/gsa-058-vor-1-with-glideslopeindicator/. Accessed: 2016, April 27. 
[126] Transport Canada. Aeronautical Information Manual - AIM 2016-1, chapter AIR 4.11. Transport Canada, March 2016. TP 14371E.

[127] W23/W31 series: Toggle or Push/Pull Actuator Thermal P\&B Circuit Breaker. Available: http://www.te.com/catalog/pn/en/6-1393246-3. Accessed: 2015, February 13.

[128] Series 16 Push-to-Reset. Available: http://www.mechprod.com/engineersresource-2/data-sheets/. Accessed: 2015, February 13.

[129] LabJack T7 Datasheet: Appendix A - Specifications. Available: https:// labjack.com/support/datasheets/t7/appendix-a. Accessed: 2016, February 3 .

[130] SN54159, SN74159 4 Line to 16 Line Decoders/Demultiplexers with OpenCollector Outputs. Available: http://www.ti.com/product/sn74159. Accessed: 2016, February 3.

[131] CESSNA 172 SP version 1.52 released! Available: http://www.airfoillabs. com/. Accessed: 2016, May 2.

[132] Cessna 172SP Skyhawk. Available: http://store.x-plane.org/Cessna172SP-Skyhawk_p_401.html. Accessed: 2016, May 2.

[133] H. Rajaee. Development of multifarious cueing systems for cost-effective flight simulation training devices. Master's thesis, Carleton University, 2016.

[134] HT1085ST [Canadian website]. Available: http://www.benq.ca/product/ projector/ht1085st/. Accessed: 2016, May 21.

[135] How TI DLP technology works. Available: http://www.ti.com/lsds/ti/dlptechnology/about-dlp-technology/how-dlp-technology-works . page. Accessed: 2016, May 21.

[136] S. Poretsky. How the Color Wheel on a Projector Works. Available: http: //yourbusiness azcentral .com/color-wheel-projector-works2753.html. Accessed: 2016, May 21.

[137] The Rainbow Effect. Available: http://www.epson.com/cgi-bin/Store/ jsp/Landing/projector-rainbow-effect-visual-artifacts.do. Accessed: 2016, May 21. 
[138] HT1085ST [American website]. Available: http://www2.benq.us/product/ projector/ht1085st/. Accessed: 2016, May 21.

[139] Fly Elise-ng Homepage. Available: http://fly.elise-ng.net/. Accessed: 2016, May 21.

[140] ButtKicker Simulation Kit. Available: http://www.thebuttkicker.com/ simulation-kit. Accessed: 2016, May 21.

[141] X-Plane Control Pad. Available: https://itunes.apple.com/us/app/xplane-control-pad/id961801382?ls=1\&mt=8. Accessed: 2016, May 23.

[142] Redbird SD: High Value Immersive Simulation. Available: http:// simulators.redbirdflight.com/products/sd. Accessed: 2016, June 30.

[143] TRC Simulators Price List. Available: http://www.trcsimulators.com/wpcontent/uploads/2015/03/Catalogue-Euro-Prices.pdf. Accessed: 2016, July 27. 


\section{Appendix A}

\section{History and Present Status of Diamond-Vector FTDs}

In the beginning, Diamond Aircraft (Austria) created a flight training device for their DA-20 Katana aircraft, a two-seat light aircraft suitable for flight training. It appears that an Austrian company was retained to do the actual work: Luftfahrtstudio Jungwirth, named after its proprietor Helmut Jungwirth.

As it turned out, the Katana flight training devices which were produced were too expensive compared with the actual aircraft, and Diamond sold their simulator business to Vector Training Systems, Inc. (VTS) in the United States, a company formed by three professors from the University of North Dakota (UND). As of this writing, the Department of Aviation at UND operates numerous simulators and maintains the largest fleet of collegiate training aircraft in the world.

VTS subsequently developed the Katana technology into a number of different flight training devices (FTD), including a Cessna 182 (once used by the Department of Homeland Security in the United States), a Piper Warrior, and Cessna 172.

The FTD redeveloped and modernized in this project was the first Cessna 172 FTD ever built by VTS, serial number 00. This assertion is supported by the serial number as well as anecdotally by the fact that certain components were found to 
be mounted on a prototyping perfboard instead of a mass-produced printed circuit board (PCB). Unfortunately, VTS succumbed to bankruptcy before the only other Cessna 172 FTD under construction was completed.

As the developer of the original hardware and software used in the Katana FTD, Luftfahrtstudio Jungwirth was also involved in the subsequent production of simulators by VTS. While Jungwirth's company outlasted VTS, it also eventually went bankrupt in 2010. As a result, detailed documentation for certain hardware components such as the digital output interface (DOIF) board and simulated radio stack were not available.

Jungwirth supplied parts and expertise to VTS as simulators were built in the USA. It appears that Luftfahrtstudio Jungwirth was also responsible for writing the transputer software which ran the flight model and handled cockpit input/output. The software was written in "occam," the native language of the transputer, and commented in German. A large quantity of occam source code files were included in documents obtained from Jon Roloff, former lead electronics technician at VTS.

In the course of this background research, several used examples of the original DA-20 Katana FTD by Diamond were found to have been sold by a German company, FTD Consulting. Images from the online advertisement are shown in Figure ??.

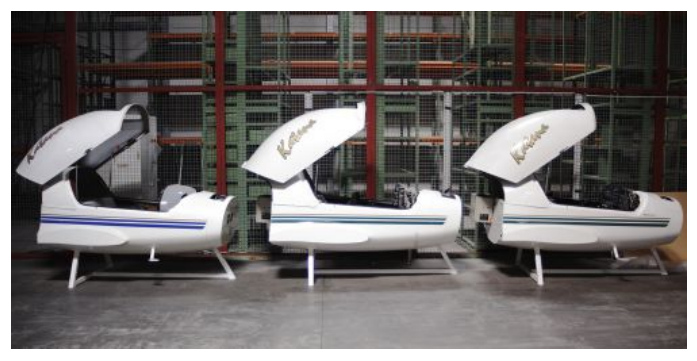

(a) Side view.

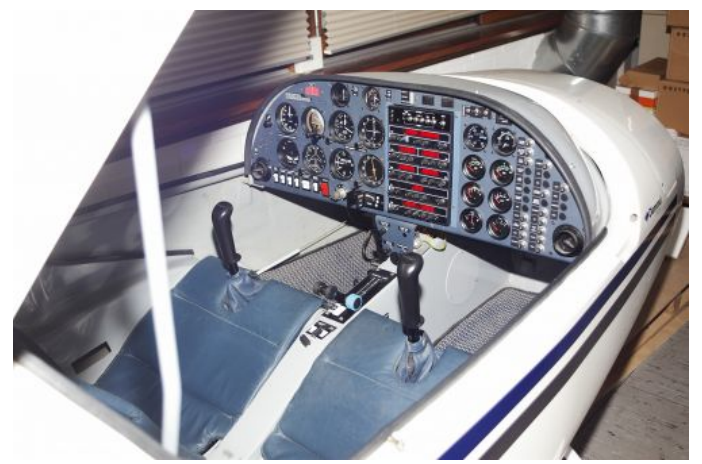

(b) Cockpit view.

Figure A.1: Original DA-20 Katana FTDs by Diamond Aircraft. Images courtesy FTD Consulting. 
Through FTD Consulting, the author was put in touch with a company called Euramec, who have retrofitted a number of original Diamond Katana FTD cockpits, installing their own electronics to create new FTDs. The original Diamond FTDs were obtained for approximately 5,000-10,000 EUR, while the retrofitted devices now retail for upwards of 42,500 EUR before certification. The Euromec device is interesting for the following reasons:

- It runs X-Plane from a single PC.

- It features a horizontal FOV of up to $230-240^{\circ}$ using triple-channel projection.

- It runs custom warping software developed in conjunction with a local university.

- The original, visually-restrictive fibreglass canopy shroud has been removed.

- The original simulated radio stack has been replaced by an LCD screen.

- It uses springs to provide approximate control loading.

\section{A.1 Further Reading}

- UND Department of Aviation: http://aviation.und.edu/

- Luftfahrtstudio Jungwirth bankruptcy (German): http://firmenbuchauszug. at/Firmenbuchinformation/2352FC09F9931D

- Online advertisement for original Diamond FTDs: http://www.jet-scout. com/Diamond-DA+20+Katana-for_sale_470.html

- FTD Consulting: http://www.ftd-consulting.com

- Euramec retrofitted DA-20 FTD: http://euramec.com/products/flighttraining-devices/eur-ftd-da20k/

- Euramec backstory (Dutch): http://www.hangarflying.eu/nl/content/ diamond-sim-voor-professionals-en-liefhebbers 


\section{Appendix B}

\section{"Teensy" Microcontroller Joystick}

\section{Interface Code}

The following code for the Arduino-compatible "Teensy" microcontroller board allows connected potentiometers to appear as joystick axes on a connected PC. No additional software is required on the PC.

2 USB 4-Axis Demo (Enables USB joystick $X, Y, Z$, and $Z$ rotation axes)

3 by BRENT CAMERON, Sept. 12, 2014.

4 Designed for: Teensy 2.0 microcontroller available from PJRC.com

5 Based on: "Basic USB Joystick Example" in the public domain, available at: https://www.pjrc.com/teensy/td_joystick.html

7 Connect potentiometer wipers (centre pins) to the following Teensy analog pins:

$8 \quad 0$ (AO, labelled FO on board) - Roll (X)

91 (A1, labelled F1 on board) - Pitch $(y)$

102 (A2, labelled F4 on board) - Throttle (Z)

113 (A3, labelled F5 on board) - Yaw (Z rotation)

12

13 You must select "Keyboard + Mouse + Joystick" from the "Tools > USB Type" menu

14

15 NOTE: FlightGear users must assign the rudder axis in "Help > Joystick Configuration" from within the program. No additional configuration is necessary for Microsoft Flight Simulator 2002 Professional, 2004, X ( demo), or Microsoft Flight. 
16

17 Pushbuttons should be connected to digital pins 0 and 1 . Wire each button between the digital pin and ground. Potentiometers should be connected to analog inputs 0 to 3 (default).

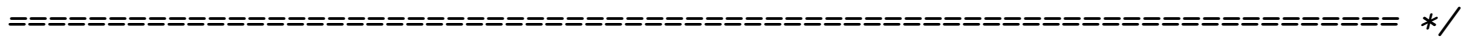




\section{Appendix $\mathrm{C}$}

\section{X-Plane UDP Datagram Output Format}

X-Plane can output a subset of its available datarefs to a specified IP address on a computer network in the form of UDP packets. The desired datasets may be selected from the X-Plane menu: Settings > Data Input \& Output. In addition, the UDP packet transmission rate can also be selected. UDP packets are known as datagrams. Each datagram consists of a series of bytes, with the length of the datagram dependent on the total number of datasets selected. Each datagram contains the selected datasets in ascending numerical order. While each dataset contains space for up to eight variables, all eight are not necessarily used. Unused variables are denoted by the value -999 .

Each variable is represented by a 32-bit floating-point number, expressed as four bytes. For example, the four bytes which represent the 32-bit floating-point dummy value -999 are shown in Table C.1.

Table C.1: X-Plane dummy value in byte form

\begin{tabular}{|l|c|c|c|c|}
\hline Dummy Value & \multicolumn{4}{|c|}{-999} \\
\hline & Byte 1 & Byte 2 & Byte 3 & Byte 4 \\
\hline Decimal Byte Representation & 0 & 192 & 121 & 196 \\
\hline Binary Byte Representation & 00000000 & 11000000 & 01111001 & 11000100 \\
\hline
\end{tabular}


The X-Plane UDP datagram is structured as shown in Table C.2. The prologue appears at the beginning of each datagram, followed by the number of the lowest dataset, the eight variables contained in that dataset, the number of the next-greatest dataset, the eight variables contained in that dataset, etc.

Table C.2: X-Plane datagram structure

\begin{tabular}{|c|c|c|c|c|c|c|c|c|c|c|}
\hline Prologue & Dataset \# & \multicolumn{8}{|c|}{ Dataset $n$ Variables } & \\
\hline DATA@ & $n 000$ & float1 & float2 & float3 & float4 & float5 & float6 & float7 & float8 & $\ldots$ \\
\hline 5 Bytes & 4 Bytes & \multicolumn{8}{|c|}{$8 \times 4$ Bytes $=32$ Bytes } & \\
\hline
\end{tabular}

The length of each datagram is dependant on the total number of datasets selected for output according to the following formula:

$$
\text { Length }=5 \text { Bytes }+(\text { Number of Datasets Selected }) \times(4 \text { Bytes }+32 \text { Bytes })
$$

The prologue of each datagram is five bytes long, and structured as shown in Table C.3. These five bytes correspond to characters in the American Standard Code for Information Interchange (ASCII).

Table C.3: X-Plane datagram prologue

\begin{tabular}{|l|c|c|c|c|c|}
\hline & \multicolumn{5}{|c|}{ Prologue } \\
\hline ASCII Byte Representation & D & A & T & A & @ \\
\hline Decimal Byte Representation & 68 & 65 & 84 & 65 & 64 \\
\hline Size & 1 Byte & 1 Byte & 1 Byte & 1 Byte & 1 Byte \\
\hline
\end{tabular}

\section{C.1 C\# and UDP}

The C\# programming language provides several methods (class member functions) which make it straightforward to receive and process UDP datagrams from X-Plane. 
For example, the method BitConverter.ToSingle() may be used to easily convert groups of four bytes received from X-plane into their single-precision floating-point decimal equivalents.

\section{C.2 Further Reading}

The following resources contain more information regarding the X-Plane UDP format and how to interpret it.

- "Communicating with X-Plane using C\# and UDP," available: http://www . nuclearprojects.com/xplane/

- "Barron 58 Cockpit Simulator: Data Input/Output," available: http://b58. svglobe.com/data.html 


\section{Appendix D}

\section{X-Plane UDP Dataset Variables}

The individual variables available in each UDP dataset are documented in the manual

which ships with X-Plane; however, the presence of blank fields within the datasets are not. Thus, in order to determine the actual sequence of variables, the desired datasets must first be selected for on-screen display and their contents visually inspected. Not content with this approach, the author has compiled a complete list of all X-Plane UDP datasets, the fields contained within each, and the order in which they appear. This data is given below, grouped as shown in the X-Plane "Data Input \& Output" menu. 


\begin{tabular}{|c|c|c|c|c|c|c|c|c|c|}
\hline Index & Data Set Name & Field 1 & Field 2 & Field 3 & Field 4 & Field 5 & Field 6 & Field 7 & Field 8 \\
\hline $\begin{array}{l}0 \\
1 \\
2\end{array}$ & $\begin{array}{l}\text { frame rate } \\
\text { times } \\
\text { sim stats }\end{array}$ & $\begin{array}{l}\text { f-act, /sec } \\
\text { real, time } \\
\text { explo, DIM }\end{array}$ & $\begin{array}{l}\text { f-sim, /sec } \\
\text { totl, time } \\
\text { explo, USE }\end{array}$ & $\begin{array}{l}\text { missn, time } \\
\text { cratr, DIM }\end{array}$ & $\begin{array}{l}\text { frame, time } \\
\text { timer, time } \\
\text { cratr, USE }\end{array}$ & $\begin{array}{l}\text { cpu, time } \\
\text { puffs, TOT }\end{array}$ & $\begin{array}{l}\text { gpu, time } \\
\text { zulu, time } \\
\text { puffs, VIS }\end{array}$ & $\begin{array}{l}\text { grnd, ratio } \\
\text { local, time } \\
\text { tris, vis }\end{array}$ & $\begin{array}{l}\text { flit, ratio } \\
\text { hobbs,time } \\
\text { q,depth }\end{array}$ \\
\hline $\begin{array}{l}3 \\
4\end{array}$ & $\begin{array}{l}\text { speeds } \\
\text { Mach, VVI, G-load }\end{array}$ & $\begin{array}{l}\text { Vind, kias } \\
\text { Mach, ratio }\end{array}$ & Vind, keas & $\begin{array}{l}\text { Vtrue, ktas } \\
\text { VVI, fpm }\end{array}$ & Vtrue, ktgs & Gload, norml & $\begin{array}{l}\text { Vind, mph } \\
\text { Gload,axial }\end{array}$ & $\begin{array}{l}\text { Vtrue, mphas } \\
\text { Gload, side }\end{array}$ & Vtrue, mphgs \\
\hline $\begin{array}{l}5 \\
6 \\
7\end{array}$ & $\begin{array}{l}\text { Atmosphere: weather } \\
\text { Atmosphere: Aircraft } \\
\text { system pressures }\end{array}$ & $\begin{array}{l}\text { SLprs, inHG } \\
\text { AMprs, inHG } \\
\text { baro, inHG }\end{array}$ & $\begin{array}{l}\text { SLtmp, } \operatorname{deg} \mathrm{C} \\
\text { AMtmp, degC } \\
\text { edens, part }\end{array}$ & $\begin{array}{l}\text { LEtmp, } \operatorname{deg} C \\
\text { vacum, ratio }\end{array}$ & $\begin{array}{l}\text { wind, speed } \\
\text { dens, ratio } \\
\text { vacum, ratio }\end{array}$ & $\begin{array}{l}\text { wind, dir } \\
\text { A, ktas } \\
\text { elec, ratio }\end{array}$ & $\begin{array}{l}\text { trb, locl } \\
\text { Q, psf } \\
\text { elec, ratio }\end{array}$ & $\begin{array}{l}\text { prec, locl } \\
\text { AHRS, ratio }\end{array}$ & $\begin{array}{l}\text { hail, locl } \\
\text { gravi, fts2 } \\
\text { AHRS, ratio }\end{array}$ \\
\hline $\begin{array}{l}8 \\
9 \\
10 \\
11 \\
\end{array}$ & $\begin{array}{l}\text { joystick ail/elv/rud } \\
\text { other flight controls } \\
\text { art stab ail/elv/rud } \\
\text { flight con ail/elv/rud }\end{array}$ & $\begin{array}{l}\text { elev, yoke1 } \\
\text { vect, rqst } \\
\text { elev, astab } \\
\text { elev, surf } \\
\end{array}$ & $\begin{array}{l}\text { ailrn, yoke1 } \\
\text { sweep, rqst } \\
\text { ailrn, astab } \\
\text { ailrn, surf } \\
\end{array}$ & $\begin{array}{l}\text { ruddr, yoke1 } \\
\text { incid, rqst } \\
\text { ruddr, astab } \\
\text { ruddr, surf } \\
\end{array}$ & dihed, rqst & $\begin{array}{l}\text { retra, rqst } \\
\text { nwhel, steer }\end{array}$ & & & water, jetts \\
\hline $\begin{array}{l}12 \\
13 \\
14 \\
\end{array}$ & $\begin{array}{l}\text { wing sweep/thrust vect } \\
\text { trim/flap/slat/s-brakes } \\
\text { gear/brakes }\end{array}$ & $\begin{array}{l}\text { sweep, } 1 \text {, deg } \\
\text { trim, elev } \\
\text { gear, 0/1 }\end{array}$ & $\begin{array}{l}\text { sweep, } 2, \text { deg } \\
\text { trim, ailrn } \\
\text { wbrak, part }\end{array}$ & $\begin{array}{l}\text { sweep,h, deg } \\
\text { trim, ruddr } \\
\text { lbrak, add }\end{array}$ & $\begin{array}{l}\text { vect, ratio } \\
\text { flap, handl } \\
\text { rbrak, add }\end{array}$ & $\begin{array}{l}\text { sweep, ratio } \\
\text { flap, postn }\end{array}$ & $\begin{array}{l}\text { incid, ratio } \\
\text { slat, ratio }\end{array}$ & $\begin{array}{l}\text { dihed, ratio } \\
\text { sbrak, handl }\end{array}$ & $\begin{array}{l}\text { retra, ratio } \\
\text { sbrak, postn }\end{array}$ \\
\hline $\begin{array}{l}15 \\
16 \\
17 \\
18 \\
19\end{array}$ & $\begin{array}{l}\text { angular moments } \\
\text { angular velocities } \\
\text { pitch, roll, headings } \\
\text { AoA, side-slip, paths } \\
\text { mag compass }\end{array}$ & $\begin{array}{l}\text { M, ftlb } \\
\text { Q,rad/s } \\
\text { pitch, deg } \\
\text { alpha, deg } \\
\text { mag, comp }\end{array}$ & $\begin{array}{l}\mathrm{L}, \mathrm{ftlb} \\
\mathrm{P}, \mathrm{rad} / \mathrm{s} \\
\text { roll, deg } \\
\text { beta, deg } \\
\text { mavar, deg }\end{array}$ & $\begin{array}{l}\mathrm{N}, \mathrm{ftlb} \\
\mathrm{R}, \mathrm{rad} / \mathrm{s} \\
\text { hding, true } \\
\text { hpath, deg }\end{array}$ & $\begin{array}{l}\text { hding, mag } \\
\text { vpath, deg }\end{array}$ & & & & slip, deg \\
\hline $\begin{array}{l}20 \\
21\end{array}$ & $\begin{array}{l}\text { lan, lon, altitude } \\
\text { loc, vel, dist traveled }\end{array}$ & $\begin{array}{l}\text { lat, deg } \\
\mathrm{X}, \mathrm{m}\end{array}$ & $\begin{array}{l}\text { lon, deg } \\
\mathrm{Y}, \mathrm{m}\end{array}$ & $\begin{array}{l}\text { alt, ftmsl } \\
\mathrm{Z}, \mathrm{m}\end{array}$ & $\begin{array}{l}\text { alt, ftagl } \\
\mathrm{vX}, \mathrm{m} / \mathrm{s}\end{array}$ & $\begin{array}{l}\text { on, runwy } \\
\mathrm{vY}, \mathrm{m} / \mathrm{s}\end{array}$ & $\begin{array}{l}\text { alt, ind } \\
\mathrm{vZ}, \mathrm{m} / \mathrm{s}\end{array}$ & $\begin{array}{l}\text { lat, south } \\
\text { dist, } \mathrm{ft}\end{array}$ & $\begin{array}{l}\text { lon, west } \\
\text { dist, } \mathrm{nm}\end{array}$ \\
\hline $\begin{array}{l}22 \\
23 \\
24 \\
\end{array}$ & $\begin{array}{l}\text { all planes: lat } \\
\text { all planes: lon } \\
\text { all planes: alt }\end{array}$ & $\begin{array}{l}\text { lat_1, deg } \\
\text { lon_1, deg } \\
\text { alt_1, ftmsl }\end{array}$ & $\begin{array}{l}\text { lat_2, deg } \\
\text { lon_2, deg } \\
\text { alt_2, ftmsl }\end{array}$ & $\begin{array}{l}\text { lat_3, deg } \\
\text { lon_3, deg } \\
\text { alt_3, ftmsl }\end{array}$ & $\begin{array}{l}\text { lat_4, deg } \\
\text { lon_4, deg } \\
\text { alt_4, ftmsl }\end{array}$ & $\begin{array}{l}\text { lat_5, deg } \\
\text { lon_5, deg } \\
\text { alt_5, ftmsl }\end{array}$ & $\begin{array}{l}\text { lat_6, deg } \\
\text { lon_6, deg } \\
\text { alt_6, ftmsl }\end{array}$ & $\begin{array}{l}\text { lat_7, deg } \\
\text { lon_7, deg } \\
\text { alt_7, ftmsl }\end{array}$ & $\begin{array}{l}\text { lat_8, deg } \\
\text { lon_8, deg } \\
\text { alt_8, ftmsl }\end{array}$ \\
\hline $\begin{array}{l}25 \\
26 \\
27 \\
28 \\
29 \\
30 \\
31 \\
32\end{array}$ & $\begin{array}{l}\text { throttle command } \\
\text { throttle actual } \\
\text { feathr-norm-beta-revers } \\
\text { prop setting } \\
\text { mixture setting } \\
\text { carb heat setting } \\
\text { cowl flap setting } \\
\text { magneto setting }\end{array}$ & $\begin{array}{l}\text { thro1, part } \\
\text { thro1, part } \\
\text { mode1, 0123 } \\
\text { prop1, set } \\
\text { mixt1, ratio } \\
\text { heat1, ratio } \\
\text { cowl1, set } \\
\text { magn1, set }\end{array}$ & & & & & & & \\
\hline $\begin{array}{l}33 \\
34 \\
35 \\
36 \\
37 \\
38 \\
39\end{array}$ & $\begin{array}{l}\text { starter timeout } \\
\text { engine power } \\
\text { engine thrust } \\
\text { engine torgue } \\
\text { engine RPM } \\
\text { prop RPM } \\
\text { prop pitch }\end{array}$ & $\begin{array}{l}\text { star1, sec } \\
\text { power, 1,hp } \\
\text { thrst, 1,lb } \\
\text { trq 1, ftlb } \\
\text { rpm 1, engin } \\
\text { rpm 1, prop } \\
\text { ptch1, deg }\end{array}$ & & & & & & & \\
\hline
\end{tabular}




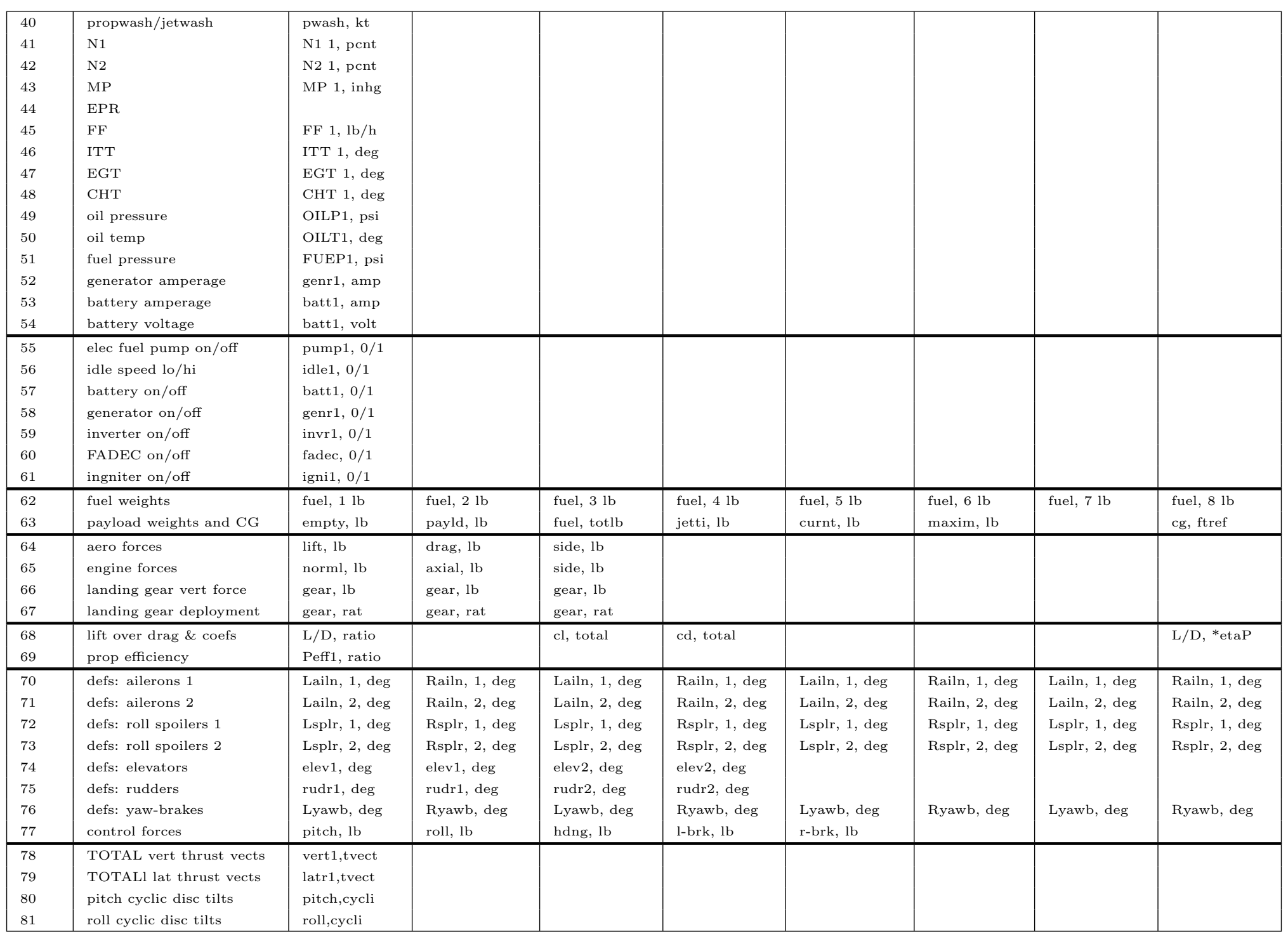




\begin{tabular}{|c|c|c|c|c|c|c|c|c|c|}
\hline $\begin{array}{l}82 \\
83\end{array}$ & $\begin{array}{l}\text { pitch cyclic flapping } \\
\text { roll cyclic flapping }\end{array}$ & $\begin{array}{l}\text { pitch, flap } \\
\text { roll, flap }\end{array}$ & & & & & & & \\
\hline $\begin{array}{l}84 \\
85 \\
86 \\
87 \\
88 \\
89 \\
90 \\
91\end{array}$ & $\begin{array}{l}\text { grnd effect lift, wings } \\
\text { grnd effect drag, wings } \\
\text { grnd effect wash, wings } \\
\text { grnd effect lift, stabs } \\
\text { grnd effect, drag,stabs } \\
\text { grnd effect wash, stabs } \\
\text { grnd effect lift, props } \\
\text { grnd effect drag, props }\end{array}$ & $\begin{array}{l}\text { wing1, L cl* } \\
\text { wing1, Lcdi* } \\
\text { wing1, wash* } \\
\text { hstab, L cl* } \\
\text { hstab, Lcdi* } \\
\text { hstab, wash* } \\
\text { prop1, cl* } \\
\text { prop1, cdi* }\end{array}$ & $\begin{array}{l}\text { wing1,R cl* } \\
\text { wing1,Rcdi* } \\
\text { wing1,wash* } \\
\text { hstab,R cl* } \\
\text { hstab,Rcdi* } \\
\text { hstab,wash* }\end{array}$ & $\begin{array}{l}\text { wing2,L cl* } \\
\text { wing2,Lcdi* } \\
\text { wing2, wash* } \\
\text { vstb1, cl* } \\
\text { vstb1, cdi* } \\
\text { vstb1, wash* }\end{array}$ & $\begin{array}{l}\text { wing2, } \mathrm{R} \mathrm{cl}{ }^{*} \\
\text { wing2, Rcdi* } \\
\text { wing2, wash* } \\
\text { vstb2, cl* } \\
\text { vstb2, cdi* } \\
\text { vstb2, wash* }\end{array}$ & & & & \\
\hline $\begin{array}{l}92 \\
93 \\
94 \\
95\end{array}$ & $\begin{array}{l}\text { wing lift } \\
\text { wing drag } \\
\text { stab lift } \\
\text { stab drag }\end{array}$ & $\begin{array}{l}\text { wing1, lift } \\
\text { wing1, drag } \\
\text { hstab, lift } \\
\text { hstab, drag }\end{array}$ & $\begin{array}{l}\text { wing1, lift } \\
\text { wing1, drag } \\
\text { hstab, lift } \\
\text { hstab, drag }\end{array}$ & $\begin{array}{l}\text { wing2, lift } \\
\text { wing2, drag } \\
\text { vstb1, lift } \\
\text { vstb1, drag }\end{array}$ & $\begin{array}{l}\text { wing2, lift } \\
\text { wing2, drag } \\
\text { vstb2, lift } \\
\text { vstb2, drag }\end{array}$ & & & & \\
\hline $\begin{array}{l}96 \\
97 \\
98 \\
99 \\
100 \\
101 \\
102 \\
103 \\
104 \\
105 \\
\end{array}$ & $\begin{array}{l}\text { COM } 1 / 2 \text { frequency } \\
\text { NAV } 1 / 2 \text { frequency } \\
\text { NAV } 1 / 2 \text { OBS } \\
\text { NAV } 1 \text { deflections } \\
\text { NAV } 2 \text { deflections } \\
\text { ADF } 1 / 2 \text { status } \\
\text { DME status } \\
\text { GPS status } \\
\text { XPNDR status } \\
\text { MARKER status }\end{array}$ & $\begin{array}{l}\text { COM 1, freq } \\
\text { NAV 1, freq } \\
\text { NAV 1, OBS } \\
\text { NAV 1, n-typ } \\
\text { NAV 2, n-typ } \\
\text { ADF 1, freq } \\
\text { DME, nav01 } \\
\text { GPS, mode } \\
\text { trans, mode } \\
\text { OM, morse }\end{array}$ & $\begin{array}{l}\text { COM 1, stby } \\
\text { NAV 1, stby } \\
\text { NAV 1, s-crs } \\
\text { NAV 1, to-fr } \\
\text { NAV 2, to-fr } \\
\text { ADF 1, card } \\
\text { DME, mode } \\
\text { GPS,index } \\
\text { trans, sett } \\
\text { MM,morse }\end{array}$ & $\begin{array}{l}\text { NAV 1, type } \\
\text { NAV 1, flag } \\
\text { NAV 1, m-crs } \\
\text { NAV 2, m-crs } \\
\text { ADF 1, r-brg } \\
\text { DME, found } \\
\text { dist, nm } \\
\text { trans, ID } \\
\text { IM, morse }\end{array}$ & $\begin{array}{l}\text { NAV 1, r-brg } \\
\text { NAV 2, r-brg } \\
\text { ADF } 1, \text { n-typ } \\
\text { DME, dist } \\
\text { OBS, mag } \\
\text { trans, inter } \\
\text { audio, activ }\end{array}$ & $\begin{array}{l}\text { COM_2, stby } \\
\text { NAV_2, freq } \\
\text { NAV_2, OBS } \\
\text { NAV_1, dme-d } \\
\text { NAV_2, dme-d } \\
\text { ADF_2, freq } \\
\text { DME, speed } \\
\text { crs, mag }\end{array}$ & $\begin{array}{l}\text { NAV_2, stby } \\
\text { NAV_2, s-crs } \\
\text { NAV_1, h-def } \\
\text { NAV_2, h-def } \\
\text { ADF_2, card } \\
\text { DME, time } \\
\text { rel, brng }\end{array}$ & $\begin{array}{l}\text { COM, xmt } \\
\text { NAV 2, type } \\
\text { NAV 2, flag } \\
\text { NAV 1, v-def } \\
\text { NAV 2, v-def } \\
\text { ADF 2,r-brg } \\
\text { DME, n-typ } \\
\text { hdef, dots }\end{array}$ & $\begin{array}{l}\text { ADF_2, n-typ } \\
\text { DME-3, freq } \\
\text { vdef, dots }\end{array}$ \\
\hline $\begin{array}{l}106 \\
107 \\
108 \\
109 \\
110 \\
111 \\
112 \\
\end{array}$ & $\begin{array}{l}\text { switches 1: electrical } \\
\text { switches 2: EFIS } \\
\text { switches 3: AP/f-dir/HUD } \\
\text { switches 4: anti-ice } \\
\text { switches 5: anti-ice/fuel } \\
\text { switches 6: clutch/astab } \\
\text { switches 7: misc } \\
\end{array}$ & $\begin{array}{l}\text { avio, } 0 / 1 \\
\text { ECAM, mode } \\
\text { ap, src } \\
\text { deice, all } \\
\text { alt, air0 } \\
\text { prero,engag } \\
\text { tot,energ } \\
\end{array}$ & $\begin{array}{l}\text { nav, lite } \\
\text { EFIS, sel } 1 \\
\text { fdir, mode } \\
\text { deice, inlet } \\
\text { alt, air1 } \\
\text { prero,engag } \\
\text { radal, feet } \\
\end{array}$ & $\begin{array}{l}\text { beacn, lite } \\
\text { EFIS, sel } 2 \\
\text { fdir, ptch } \\
\text { deice, prop } \\
\text { auto, ignit } \\
\text { clutc,ratio } \\
\text { prop, sync } \\
\end{array}$ & $\begin{array}{l}\text { strob, lite } \\
\text { HSI, sel } 1 \\
\text { fdir, roll } \\
\text { deice, windo } \\
\text { auto, ignit } \\
\text { clutc,ratio } \\
\text { fethr, mode } \\
\end{array}$ & $\begin{array}{l}\text { land, lite } \\
\text { HSI, sel_2 } \\
\text { deice, pito1 } \\
\text { manul, ignit } \\
\text { art, ptch } \\
\text { puffr, power } \\
\end{array}$ & $\begin{array}{l}\text { taxi, lite } \\
\text { HSI, arc } \\
\text { HUD, power } \\
\text { deice, pito2 } \\
\text { manul,ignit } \\
\text { art, roll } \\
\text { water, scoop } \\
\end{array}$ & $\begin{array}{l}\text { map, r-sel } \\
\text { HUD, brite } \\
\text { deice, AOA } \\
\text { l-eng, tank } \\
\text { yaw, damp } \\
\text { arrst, hook }\end{array}$ & $\begin{array}{l}\text { map, range } \\
\text { deice, wing } \\
\text { r-eng, tank } \\
\text { auto, brake } \\
\text { chute, deply }\end{array}$ \\
\hline $\begin{array}{l}113 \\
114 \\
115 \\
\end{array}$ & $\begin{array}{l}\text { annunciators: general } \\
\text { annunciators: general } \\
\text { annunciators: engine }\end{array}$ & $\begin{array}{l}\text { mast, cau } \\
\text { yawda, on } \\
\text { fuel, press }\end{array}$ & $\begin{array}{l}\text { mast, war } \\
\text { sbrk, on } \\
\text { oil, press }\end{array}$ & $\begin{array}{l}\text { mast, accp } \\
\text { GPWS, warn } \\
\text { oil, temp }\end{array}$ & $\begin{array}{l}\text { auto, disco } \\
\text { ice, warn } \\
\text { inver, warn }\end{array}$ & $\begin{array}{l}\text { low, vacum } \\
\text { pitot, off } \\
\text { gener, warn }\end{array}$ & $\begin{array}{l}\text { low, volt } \\
\text { cabin, althi } \\
\text { chip, detec }\end{array}$ & $\begin{array}{l}\text { fuel, quant } \\
\text { afthr, arm } \\
\text { engin, fire }\end{array}$ & $\begin{array}{l}\text { hyd, press } \\
\text { ospd, time } \\
\text { ignit, } 0 / 1\end{array}$ \\
\hline $\begin{array}{l}116 \\
117 \\
118\end{array}$ & $\begin{array}{l}\text { autopilot arms } \\
\text { autopilot modes } \\
\text { autopilot values }\end{array}$ & $\begin{array}{l}\text { nav, arm } \\
\text { auto,throt } \\
\text { set,speed }\end{array}$ & $\begin{array}{l}\text { alt, arm } \\
\text { mode, hding } \\
\text { set, hding }\end{array}$ & $\begin{array}{l}\text { app, arm } \\
\text { mode, alt } \\
\text { set, vvi }\end{array}$ & $\begin{array}{l}\text { vnav, enab } \\
\text { dial, alt }\end{array}$ & $\begin{array}{l}\text { vnav, arm } \\
\text { bac, } 0 / 1 \\
\text { vnav, alt }\end{array}$ & $\begin{array}{l}\text { vnav, time } \\
\text { app, } \\
\text { use, alt }\end{array}$ & $\begin{array}{l}\text { gp, enabl } \\
\text { sync, roll }\end{array}$ & $\begin{array}{l}\text { app, typ } \\
\text { sync, butn } \\
\text { sync, pitch }\end{array}$ \\
\hline $\begin{array}{l}119 \\
120 \\
121 \\
122 \\
123\end{array}$ & $\begin{array}{l}\text { weapon status } \\
\text { pressurization status } \\
\text { APU/GPU status } \\
\text { radar status } \\
\text { hydraulic status }\end{array}$ & $\begin{array}{l}\text { hdng, delta } \\
\text { alt, set } \\
\text { APU, eqipd } \\
\text { targ, selct } \\
\text { hydr, pump1 }\end{array}$ & $\begin{array}{l}\text { ptch, delta } \\
\text { vvi, set }\end{array}$ & $\begin{array}{l}\mathrm{R}, \mathrm{d} / \mathrm{sec} \\
\text { alt, act } \\
\text { APU, swtch } \\
\text { hydr, pump1 }\end{array}$ & $\begin{array}{l}\mathrm{Q}, \mathrm{d} / \mathrm{sec} \\
\mathrm{vvi}, \text { act } \\
\text { APU, runng }\end{array}$ & $\begin{array}{l}\text { rudd, ratio } \\
\text { test, time } \\
\text { APU, N1 } \\
\text { hydr, qty1 }\end{array}$ & $\begin{array}{l}\text { elev, ratio } \\
\text { diff, psi } \\
\text { APU, genrt } \\
\text { hydr, qty2 }\end{array}$ & $\begin{array}{l}\mathrm{V}, \mathrm{kts} \\
\text { dump, all }\end{array}$ & $\begin{array}{l}\text { dis, ft } \\
\text { bleed, src } \\
\text { GPU, power }\end{array}$ \\
\hline
\end{tabular}




\begin{tabular}{|c|c|c|c|c|c|c|c|c|c|}
\hline $\begin{array}{l}124 \\
125 \\
126 \\
127 \\
128 \\
\end{array}$ & $\begin{array}{l}\text { elec \& solar status } \\
\text { icing status } 1 \\
\text { icing status } 2 \\
\text { warning status } \\
\text { flite-plane legs } \\
\end{array}$ & $\begin{array}{l}\text { bus1, amp } \\
\text { inlet, ice } \\
\text { aoa, ice } \\
\text { warn, time } \\
\text { leg, \# } \\
\end{array}$ & $\begin{array}{l}\text { bus } 2, \text { amp } \\
\text { inlet, ice } \\
\text { aoa,ice } \\
\text { caut, time } \\
\text { leg, type } \\
\end{array}$ & $\begin{array}{l}\text { bus1, volt } \\
\text { prop, ice } \\
\text { lwing, ice } \\
\text { warn, work } \\
\text { leg, lat } \\
\end{array}$ & $\begin{array}{l}\text { bus2, volt } \\
\text { prop, ice } \\
\text { rwing, ice } \\
\text { caut, work } \\
\text { leg, lon } \\
\end{array}$ & $\begin{array}{l}\text { engin, in } \mathrm{W} \\
\text { pitot, ice } \\
\text { windo, ice } \\
\text { gear, work }\end{array}$ & $\begin{array}{l}\text { solar, out W } \\
\text { pitot, ice } \\
\text { gear, warn }\end{array}$ & $\begin{array}{l}\text { batt, w-hr } \\
\text { statc, ice } \\
\text { stall, warn }\end{array}$ & $\begin{array}{l}\text { cross, tie } \\
\text { statc, ice } \\
\text { VRS, ratio }\end{array}$ \\
\hline $\begin{array}{l}129 \\
130 \\
131\end{array}$ & $\begin{array}{l}\text { hardware options } \\
\text { camera location } \\
\text { ground location }\end{array}$ & $\begin{array}{l}\text { pedal, nobrk } \\
\text { camra, lon } \\
\text { cntr, X }\end{array}$ & $\begin{array}{l}\text { pedal, wibrk } \\
\text { camra, lat } \\
\text { cntr, Y }\end{array}$ & $\begin{array}{l}\text { yoke, } \mathrm{PFC} \\
\text { camra, ele } \\
\text { cntr, Z }\end{array}$ & $\begin{array}{l}\text { pedal, PFC } \\
\text { camra, hdng } \\
\text { slope, } X\end{array}$ & $\begin{array}{l}\text { throt, PFC } \\
\text { camra, pitch } \\
\text { slope, Z }\end{array}$ & $\begin{array}{l}\text { cecon, } \mathrm{PFC} \\
\text { camra, roll }\end{array}$ & switc, PFC & $\begin{array}{l}\text { btogg, PFC } \\
\text { camra, clou }\end{array}$ \\
\hline $\begin{array}{l}132 \\
133\end{array}$ & $\begin{array}{l}\text { climb stats } \\
\text { cruise stats }\end{array}$ & $\begin{array}{l}\text { h-spd, kt } \\
\text { ff, pph }\end{array}$ & $\begin{array}{l}\text { v-spd, fpm } \\
\text { ff, gph }\end{array}$ & speed, mph & $\begin{array}{l}\text { mult, VxVVI } \\
\text { eta, smpg }\end{array}$ & eta,nm/lb & range, sm & endur,hours & mult,VxMPG \\
\hline
\end{tabular}

